\title{
Human Hepatocellular Carcinomas with a Periportal Phenotype Have the Lowest Potential for Early Recurrence after Curative Resection
}

Romain Désert, ${ }^{1}$ Florian Rohart, ${ }^{2}$ Frédéric Canal, ${ }^{3}$ Marie Sicard, ${ }^{1}$ Mireille Desille, ${ }^{1}$ Stéphanie Renaud, ${ }^{1}$ Bruno Turlin, ${ }^{1}$ Pascale Bellaud, ${ }^{1}$ Christine Perret, ${ }^{3}$ Bruno Clément, ${ }^{1}$ Kim-Anh Lê Cao, ${ }^{2}$ Orlando Musso ${ }^{1, \#}$

${ }^{1}$ INSERM, INRA, Univ Rennes 1, Univ Bretagne Loire, CHU Pontchaillou, Nutrition Metabolisms and Cancer, CRB-Santé, Biosit, Biogenouest, Rennes, France.

${ }^{2}$ The University of Queensland, Diamantina Institute, The University of Queensland, Translational Research Institute, Brisbane, QLD, Australia;

${ }^{3}$ INSERM, CNRS, Univ Paris Descartes, Sorbonne Paris Cité, Institut Cochin, Paris, France.

Running head: Low Early Recurrence of HCCs with Periportal Phenotype

This is the author manuscript accepted for publication and has undergone full peer review but has not been through the copyediting, typesetting, pagination and proofreading process, which may lead to differences between this version and the Version record. Please cite this article as doi:10.1002/ hep.29254. 


\section{E-mail addresses:}

RD, rdesert87@gmail.com

FR, f.rohart@uq.edu.au

FC, frederic.canal94@gmail.com

MS, sicard.marie.i@gmail.com

MD, mireille.desille-dugast@chu-rennes.fr

SR, stephanie.renaud39@gmail.com

BT, bruno.turlin@chu-rennes.fr

PB, pascale.bellaud@univ-rennes1.fr

CP, christine.perret@inserm.fr

BC, bruno.clement@inserm.fr

K-A LC, k.lecao@uq.edu.au

OM, orlando.musso@inserm.fr 
Keywords: Wnt/ $\beta$-catenin; liver zonation; HNF4A; sparse multivariate discriminant analysis; HCC classification.

\section{Contact information:}

\#Corresponding author

Phone: $\quad 33-(0) 223234565$

Fax: 33-(0)2 $\quad$ 39540137

E-mail: orlando.musso@inserm.fr

List of abbreviations: APC, adenomatous polyposis coli; AUROC, area under the receiver operating curve; BIO, 6-bromoindirubin-3'-oxime; DF, disease-free survival; ECM, extracellular matrix; GSEA, gene set enrichment analysis; HAL, histidine ammonia lyase; HCC, hepatocellular carcinoma; ODAM, odontogenic ameloblastassociated protein; PCA, principal component analysis; PP, periportal; PV, perivenous; sPLS-DA, sparse partial least squares discriminant analysis; TCF, T-cell factor; VNN1, vanin 1.

Title: 115 characters,

Abstract: 275 words,

Manuscript, 5842 words,

References: 49,

Figures: 7,

\section{Tables: 1}

Supporting Figures: 15,

Supporting Tables: 12,

Pages (main manuscript): 36 . 


\section{ABSTRACT}

Hepatocellular carcinomas (HCCs) exhibit a diversity of molecular phenotypes, raising major challenges in clinical management. HCCs detected by surveillance programs at an early stage are candidates for potentially curative therapies (local ablation, resection or transplantation). In the long term, transplantation provides the lowest recurrence rates. Treatment allocation is based on tumor number, size, vascular invasion, performance status, functional liver reserve and on the prediction of early ( $<2$ years) recurrence, which reflects the intrinsic aggressiveness of the tumor. Well-differentiated, potentially low-aggressiveness tumors form the heterogeneous molecular class of non-proliferative HCCs, characterized by an approximate $50 \% \quad \beta$-catenin (CTNNB1) mutation rate. To define the clinical, pathological, molecular features and the outcome of non-proliferative HCCs, we constructed an 1133-HCC transcriptomic metadata set and validated findings in a publically available $210-\mathrm{HCC}$ RNAseq set. We show that non-proliferative HCCs preserve the zonation program that distributes metabolic functions along the portocentral axis in normal liver. More precisely, we identified two well-differentiated, nonproliferation subclasses, namely Periportal-type (wild-type CTNNB1) and Perivenoustype (mutant CTNNB1), which expressed negatively correlated gene networks. The new Periportal-type subclass represented $29 \%$ of all HCCs; expressed an HNF4Adriven gene network, which was down-regulated in mouse Hnf4a-KO mice; were early-stage tumors by BCLC, CLIP and TNM staging systems; had no macrovascular invasion and showed the lowest metastasis-specific gene expression levels and TP53 mutation rates. Also, we identified an 8-gene Periportal-type HCC signature, which was independently associated with the highest 2-year recurrence-free survival by multivariate analyses in two independent cohorts of 247 and 210 patients. 
Conclusion: Well-differentiated HCCs display mutually exclusive periportal or perivenous zonation programs. Among all HCCs, Periportal-type tumors have the lowest intrinsic potential for early recurrence after curative resection.

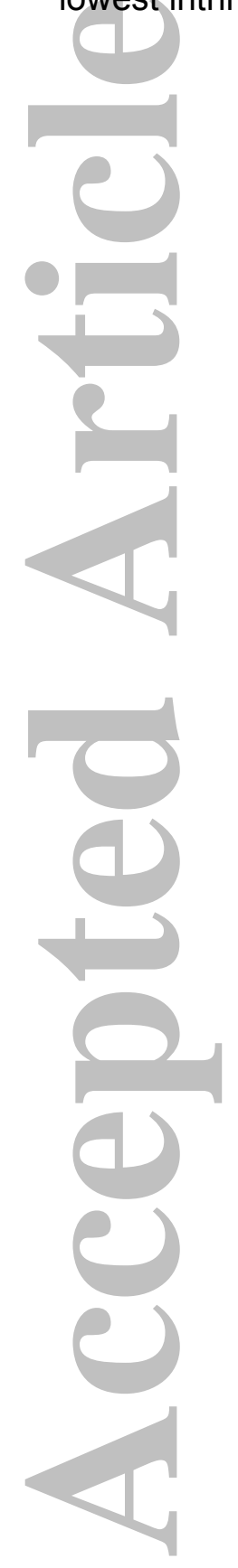

Hepatology

This article is protected by copyright. All rights reserved. 


\section{INTRODUCTION}

Hepatocellular carcinoma $(\mathrm{HCC})$ is the $3^{\text {rd }}$ leading cause of cancer-related death worldwide. HCC is endemic in East Asia and sub-Saharan Africa, and its incidence has doubled over the past 20 years in Western countries. Projections anticipate a further increase in incidence, despite recent breakthroughs in the management of chronic hepatitis.(1) More than $80 \%$ of HCCs arise in a field of chronic liver disease resulting from viral hepatitis, alcohol, hemochromatosis, obesity and metabolic syndrome or genotoxins. This diversity in etiology and natural history results in high HCC heterogeneity, raising major challenges in clinical management.(1)

Current therapeutic strategies are based upon tumor number, size, vascular invasion, performance status and functional reserve of the liver. These variables have been integrated in the Barcelona Clinic Liver Cancer (BCLC) classification to match the best candidates with the best therapies available.(1) Surveillance programs currently detect early-stage tumors (single or $\leq 3$ nodules $\leq 3 \mathrm{~cm}$ ) that are candidates for potentially, curative therapies (local ablation, resection or transplantation) with 5-year survival rates of $50-70 \%$. Five-year recurrence rates after HCC resection (up to $70 \%$ ) or even higher after percutaneous ablation make liver transplantation the best possible treatment, with a recurrence rate of $10 \%$ and the additional advantage of eliminating the underlying liver disease.(1) Treatment allocation for early-stage HCCs might be improved by identifying homogeneous molecular subclasses with predictable outcomes. $(2,3)$

The molecular landscape of HCCs is emerging as a result of global gene expression analyses and the discovery of crucial driver tumor mutations.(1, 2) At present, HCCs are split into two molecular classes, each representing $50 \%$ of tumors: proliferative 
and non-proliferative HCCs.(2) Proliferative HCCs include two subclasses enriched in WNT/TGFB signals and stem/progenitor cell markers, respectively. $(1,2)$ Among nonproliferative HCCs, half of the tumors form a homogeneous subclass with a high rate of activating $\beta$-catenin (CTNNB1) exon 3 mutations. $(2,4-6)$ These tumors are welldifferentiated, $(2,4-8)$ but patient survival rates do not differ from HCCs carrying wildtype CTNNB1 (reviewed in Supporting Table S1). The rest of non-proliferative HCCs carry wild-type CTNNB1,(5) but no evidence to date allowed them to qualify as a homogeneous HCC subclass.

This study was conducted to determine whether non-proliferative HCCs carrying wildtype CTNNB1 warrant consideration as a distinct, clinically relevant tumor subclass.

To analyze a large and statistically powerful population, we constructed an 1133HCC transcriptomic metadata set and identified four HCC subclasses by discriminant analyses and hierarchical clustering. As the 1133 HCCs were not annotated for CTNNB1 mutations, we developed a reliable method to predict CTNNB1 mutations in an independent set of $225 \beta$-catenin-sequenced HCCs. CTNNB1 mutation prediction, HCC classification and survival analyses were further validated in an external RNAseq dataset of 210 HCC patients (Fig. 1).

Altogether, analysis of data from 1568 HCC patients identified two new welldifferentiated, mutually exclusive, low-proliferation subclasses of HCCs. Both subclasses showed favorable outcomes and preserved metabolic liver zonation programs. They respectively displayed periportal (wild-type $\beta$-catenin) or perivenous (mutant $\beta$-catenin) phenotypes. Periportal-type HCCs showed the highest 2-year recurrence-free survival rates by multivariate analysis, suggesting that these tumors have the lowest potential for early recurrence among all HCCs. 


\section{Patients and Methods}

\section{Patients, study design and data management}

The study design is presented in Fig. 1. Nine public transcriptomic datasets passing the quality control analyses were integrated into a metadata set totalizing $1133 \mathrm{HCCs}$ and 9542 genes (Supporting Tables S2 and S3). As HCCs in these datasets were not annotated for CTNNB1 mutations, we developed a robust prediction method. Complying with REMARK guidelines, identification of CTNNB1 mutation predictors in a training set $(n=87)$ was confirmed in a validation set $(n=56)$ and in our recently described in-house independent cohort $(n=82) .(9)$ The three datasets (Supporting Table S2) were annotated for Sanger-sequenced CTNNB1 exon 3 mutations (Supporting Table S4). We thus predicted CTNNB1 mutational status in the 1133HCC transcriptomic metadata set. Independently, hierarchical cluster analysis was used in this metadata set to identify robust HCC subclasses. Then, the resulting tumor classification was validated in a $210-\mathrm{HCC}$ patient dataset from The Cancer Genome Atlas (TCGA) consortium(10) (normalized RNAseq genome-wide mRNA expression, whole genome sequencing and clinical data downloaded in June 2016 from the TCGA website). Out of 294 HCCs with available RNAseq and clinical annotations, 84 met our exclusion criteria (Supporting Fig. S1A). Quality control of clinical annotations was performed by studying the relationship between tumor staging and overall/disease-free survival by Kaplan-Meier plots and Log Rank tests (Supporting Fig. S1B).

Raw data from publically available transcriptome profiling experiments were extracted from Gene Expression Omnibus. Probes detected over background in at least one $\mathrm{HCC}$ were quantile normalized ( $\mathrm{R}$ package preprocessCore) and $\log _{2}$ intensity 
expression values for each probe set were calculated by Robust Multi-array Average Cross-platform and batch-dependent variances were corrected with COMBAT(11) (R package sva) in the 1133-HCC metadata set and with YuGene(12) (R package YuGene) in the datasets used for CTNNB1 mutation prediction.

\section{Statistical analyses}

To optimize the identification of stable gene predictors of CTNNB1 activating mutations, we applied Sparse Partial Least Squares Discriminant Analysis (sPLS-DA) with bootstrap subsampling (Supporting Fig. S2), as described.(13) After further marker validation in the in-house cohort by real-time PCR, we established a score to predict CTNNB1 mutations. Independently, robust HCC subclasses were identified using hierarchical cluster analysis defined by a stepwise algorithm (see Supporting Materials and Methods). Survival analyses were performed using the Log Rank test, Kaplan-Meier curves and Cox models. Statistical analyses were performed with $\mathrm{R}$ (version 3.3.0).

See Supporting Materials and Methods for: tissue banking, fully detailed statistics, functional genomics, detection of $\beta$-catenin exon 3 mutations/deletions, tissue microarray-based immunohistochemistry, antibodies, cell culture, nucleic acid extraction, real-time PCR, primers, and RNA interference. 


\section{RESULTS}

\section{A robust 5 -gene score predicts CTNNB1 mutations in large transcriptomic} datasets

As described in the Patients and Methods section and Fig. 1, nine public transcriptomic datasets were integrated into a metadata set of 1133 HCCs and 9542 genes (Supporting Tables S2 and S3). The 1133-HCC transcriptomic dataset was not annotated for CTNNB1 mutational status; thus, we set up a robust pipeline to predict CTNNB1 activating mutations (Fig. 1). The model achieved a prediction accuracy of $87 \%$ in the training set and $93 \%$ in the independent validation sets (Supporting Fig. S2). The analysis identified Histidine Ammonia-Lyase (HAL) and Vanin 1 (VNN1) (selected in $>85 \%$ of the models after 300 bootstrap runs), which were both downregulated in HCCs carrying mutant CTNNB1. In addition, a search for the top upregulated genes in HCCs carrying CTNNB1 mutations identified ODontogenic AMeloblast-associated protein (ODAM). Then, to construct a score predicting CTNNB1 mutations, we combined HAL, VNN1 and ODAM with two well-known markers of CTNNB1 activating mutations in HCCs: GLUL and LGR5.(14) RNA expression levels of the five biomarkers were significantly associated with mutant CTNNB1 in the three datasets (Fig. 2A,B). AXIN2, which reflects Wnt/ $\beta$-catenin pathway activation,(15) was positively correlated with GLUL, LGR5 and ODAM and negatively correlated with $H A L$ and VNN1 (Fig. 2A,B; Supporting Fig. S3A). Cluster analyses confirmed the association of the five markers with CTNNB1 mutation (Fig. 2, B1,B2,B3). In the external validation set, CTNNB1 mutation was negatively associated with invasive (Gamma $=-0.83, \mathrm{p}<10-3)$ and multinodular (Gamma $=-0.56$, $p=0.02$ ) tumors (Fig. 2B3). In turn, nuclear $\beta$-catenin immunostaining was positively correlated with CTNNB1 mutations, GLUL, ODAM and LGR5 and negatively with 
VNN1 and HAL and (Supporting Fig. S3B). Combination of the five markers into an arithmetic score resulted in a reliable prediction in the three datasets (AUROC $=0.87$ 0.90; Sensitivity=0.86-0.91; Specificity=0.83-1; Fig. 2C-E).

Tissue microarray-based immunohistochemistry in $20 \mathrm{HCCs}$ carrying mutant versus 20 HCCs carrying wild-type CTNNB1 revealed a clear-cut difference in GLUL expression between HCCs showing mutant versus wild-type CTNNB1 at low power microscopic examination, whereas HAL and VNN1 were globally higher in wild-type samples (Fig. 2F). ODAM protein expression was low in all samples (not shown), but detected in HCCs carrying mutant CTNNB1 and expressing high GLUL (Fig. 2G).

In the external validation set, analysis of matching HCC and non-tumor samples showed that tumor/non-tumor ratios were highly predictive of CTNNB1 mutations (AUROC=0.90, Se 0.87; Sp 0.88) (Supporting Fig. S3C-F). As data from non-tumor tissues were not available in all the transcriptomic datasets, further predictions of CTNNB1 mutations were performed with tumor samples. Thus, we predicted CTNNB1 mutations in the 1133 HCCs of the metadata set (Supporting Table S5). Predicted mutation rates between $20-40 \%$ matched the previously described CTNNB1 mutation rates in HCC.(16)

To better understand the association of the newly discovered biomarkers (HAL, VNN1 and ODAM) with CTNNB1 activating mutations, we analyzed human HCC cell lines (Supporting Fig. S4). HAL was expressed at higher levels in HCC cell lines carrying wild-type CTNNB1, i.e., Huh7 or HepaRG, than in those carrying activating CTNNB1 mutations, i.e., B16, BC2, HepG2 and Huh-6. VNN1 was expressed at much higher levels in HepaRG cells than in the cell lines carrying mutant CTNNB1. By contrast, Huh7 expressed VNN1 at low levels. Last, ODAM was clearly expressed 
at higher levels in cell lines carrying mutant than in those carrying wild-type CTNNB1. The GSK3 $\beta$ inhibitor 6-bromoindirubin-3'-oxime (BIO), which activates $\beta$-catenin signaling,(17) strongly upregulated $\beta$-catenin-dependent transcriptional activity (Supporting Fig. S5A). BIO upregulated GLUL, LGR5 and ODAM (Supporting Fig. S5B) and attenuated the increase in HNF4A, ALDOB, HAL and VNN1 mRNA expression over the 30 days required for differentiation of HepaRG progenitors to hepatocyte-like cells (Supporting Fig. S5C-F). Conversely, HepaRG cells transfected with $\beta$-catenin targeting siRNA downregulated AXIN2 and ODAM and upregulated HAL and VNN1 mRNA expression (Supporting Fig. S5G). In addition, in silico analysis of the 5000 base pairs upstream the transcription start site of ODAM DNA (NM_017855; PROMO program,(18) TRANSFAC database), revealed three putative TCF-4/LEF-1 consensus transcription factor binding sites in the ODAM 5'UTR region at positions $-99 ;-1262$ and -3956 . Taken together, these data suggest that $\beta$-catenin signaling regulates $H A L, V N N 1$ and ODAM mRNA expression.

\section{Predicted CTNNB1 mutations cluster within a homogeneous tumor subclass after analysis of transcriptomic data from 1343 HCCs.}

We integrated the nine datasets into an $1133 \mathrm{HCC}$ metadata set with 9542 common genes and performed cross-platform normalization with the COMBAT algorithm(11) (Supporting Fig. S6). Identification of the 1618 most discriminant genes by partial least squares discriminant analysis (PLS-DA), using a VIP score threshold $>1$, followed by stepwise hierarchical cluster analysis, identified four subclasses. Subclasses were named red, green, blue and purple before further functional characterization (Supporting Fig. S7). The red subclass had a predicted CTNNB1 
mutation rate of $84 \%$ (Supporting Fig. S7). The predicted CTNNB1 mutation rates in this subclass were high in all datasets $(65 \%$ to $100 \%$, Supporting Table S6). These findings are consistent with previous evidence that HCCs carrying CTNNB1 mutation are a homogeneous subclass. $(4,6)$

Reproducibility (Supporting Fig. S8) and robustness (Supporting Fig. S9A,B) analyses showed that the red and green subclasses had similarly low intra-cluster inertia, indicating high homogeneity of HCC samples within each cluster. In terms of gene expression profiles, the purple cluster was highly different from the red and green ones, whereas the blue cluster was intermediate between the green and purple ones (Supporting Fig. S9C). To further test the robustness of the HCC classification, we repeated PLS-DA-based identification of the most discriminant genes with a more stringent VIP score threshold (>1.5), which yielded 550 top discriminant genes and confirmed the four tumor subclasses (Supporting Fig. S9D). Further, the value of the 550 -geneset as a classifier was confirmed in the external validation RNAseq dataset $(n=210$ HCCs; Supporting Fig. 9E). Thus, using the top 550 genes, both the 1133-HCC transcriptomic (Fig. 3A) and the 210-HCC RNAseq (Supporting Fig. 9E) datasets confirmed four HCC subclasses and three gene sets. In the red subclass, CTNNB1 was predicted to be mutated in $89 \%$ of HCCs (Fig. 3A). In consistency with the high rate of predicted CTNNB1 mutations, the red subclass showed the highest levels of GLUL, LGR5 and ODAM and the lowest levels of VNN1 and $H A L$ (Fig. 3B).

By immunohistochemistry in human liver (Fig. 3C-D), GLUL showed a clear perivenous localization, as expected.(19) HAL showed a clear-cut periportal distribution, consistently with its expression by periportal hepatocytes in mice.(20) VNN1 was panlobular and was abundantly detected in the cytoplasm and in bile 
canaliculi in normal liver and in well-differentiated HCCs (Fig. 3C,D), which goes well with the evidence that VNN1 is one of the 59 major proteins in human bile.(21). Further, anti-VNN1 staining of bile canaliculi was totally suppressed by the immunogen polypeptide (Fig. 3D). In consistency with the above findings, full genome sequencing data from the independent RNAseq 210-HCC TCGA dataset confirmed that $81 \%$ of HCCs within the red subclass carried CTNNB1 mutations (Supporting Fig. S9E).

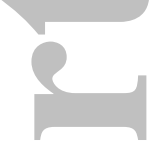

\section{Well-differentiated HCCs display a preserved metabolic liver zonation program.}

Functional genomics studies by gene set enrichment analysis (GSEA) (Fig. 4; Supporting Table S7) revealed that the HCC subclass containing the highest rate of predicted CTNNB1 mutations (84\%) was enriched in signatures of HCCs carrying mutant CTNNB1, such as the G6(6) and the CTNNB1 subclasses,(4) as well as perivenous hepatocyte signatures, such as fatty acid and bile salt metabolism.(20) We thus called this subclass "Perivenous-type HCCs" (PV). The HCC subclass enriched in signatures of differentiated periportal hepatocytes (gluconeogenesis, amino acid catabolism, HNF4A-induced genes),(20) good prognosis, (hepatocyte-like (S3) subclass HCCs)(5) and low recurrence, $(5,22)$ was called "Periportal-type HCCs" (PP). Both PP and PV subclasses were enriched in signatures of favorable survival(23) and low proliferation in HCCs.(4) The HCC subclass featuring signatures of cancer extracellular matrix (ECM) remodeling and epithelial mesenchymal transition(24) was called "ECM-type HCCs". It was characterized by integrin cellsurface interactions, KRAS, TGFB, IL6, and the MMP14 network typical of invasive tumors. The last HCC subclass was associated with high grade metastasis 
signatures, tumor aggressiveness with decreased patient survival,(23) the cancer stem cell program predicting metastasis and death(25) and upregulation of p53 mutation markers (Supporting Fig. S10). It was also enriched in signatures of cell cycle progression (MYC, RAC, AURKA, RB1 and PDGFRB pathways), HCCs displaying stem cell features(26) and extrahepatic undifferentiated cancers of various origins, as well as cancer cell resistance to doxorubicin and vincristine. Thus, we called this subclass "STEM-type HCCs". Both ECM- and STEM-type HCCs shared signatures of high tumor cell proliferation(4) and were associated with the Wnt/TGF- $\beta$ (S1) HCC subclass,(5) indicating bad prognosis.(27) Also, they were both associated with early recurrence(28). Expression levels of representative genes in HCC subclasses and in gene clusters are illustrated in Fig. 4B and in Supporting Fig. S11, respectively. Functional genomics findings were confirmed by gene ontology analysis of gene clusters (Supporting Table S8). The mean $\pm 95 \% \mathrm{Cl}$ mRNA expression level of the top 550 genes in the four HCC subclasses is shown in Supporting Table S9.

We observed differences in the percentage of samples in each subclass according to the dataset of origin (Supporting Table S10). The sampling technique (1060 resections versus 73 biopsy specimens) had no impact on tumor classification (Supporting Table S11). By contrast, cohorts containing $>95 \%$ HCV (+) HCCs showed a higher rate of PV samples than cohorts with $>95 \% \mathrm{HBV}(+)\left(p=10^{-3}\right)$, in agreement with the well-known positive association of CTNNB1 mutation with HCV and its negative association with HBV.(2)

The metabolic program of Periportal-type HCCs is regulated by HNF4A 
The interplay between TCF4, $\beta$-catenin and HNF4A(20) governs the differential distribution of metabolic liver functions along the porto-central axis of the liver, which is known as "liver zonation".(29) Using the ortholog genes of the mouse liver periportal and perivenous signatures,(20) we clustered the 326 Periportal-type and the 210 Perivenous-type HCCs from the 1133 HCC metadata set. We observed a remarkable matching of mouse normal liver perivenous genes with Perivenous-type HCCs and mouse normal liver periportal genes with Periportal-type HCCs, thus confirming the identity of both HCC subclasses described above (Supporting Fig. S12A). Subsequently, network analysis in the 1133 patient metadata set revealed that periportal and perivenous genes were negatively correlated and respectively upregulated in Periportal-type and Perivenous-type HCCs (Fig. 5A). Predictive markers of CTNNB1 mutation showed high connectivity within this network. GLUL, HAL and LGR5 were connected respectively with 60 (42\%), 54 (38\%) and 54 (38\%) of genes in the network. Although mouse Odam and Vnn1 were not present in the original periportal and perivenous signatures,(20) human ODAM and VNN1 were connected respectively with $47(33 \%)$ and $28(20 \%)$ of the genes in human HCCs.

Suppression of HNF4A in mouse liver has profound effects on zonated metabolic functions, $(30,31)$ cell proliferation(30) and oncogenesis. $(32,33)$ Consistent with this, the top 550 genes representative of the four HCC subclasses perfectly discriminated Hnf4a-KO and Hnf4a-WT(31) mouse livers (Fig. 5B). Among the 550 genes, a first cluster was strongly upregulated in Hnf4a-KO mice and clearly enriched in genes upregulated in ECM/STEM HCCs. A second cluster was downregulated in Hnf4a-KO mice and enriched in genes upregulated in Periportal-type HCCs. The rest of the genes $(20 \%)$ were poorly affected by HNF4A. Finally, we extracted from the Hnf4a$\mathrm{KO}$ dataset the genes showing the highest modulation (fold change $>2$ or $<0.5$ and 
Bonferroni adjusted $P$ value $<0.01$ ) to look at the expression of their orthologs in the 1133 HCC metadata set (Supporting Fig. S12B). Hierarchical clustering of these genes showed a good discrimination of the four subclasses $(80 \%$ of samples correctly classified), with a strong enrichment of genes highly downregulated by Hnf4a-KO in Periportal-type HCCs. Conversely, genes highly upregulated by Hnf4aKO were remarkably enriched in the STEM-type HCC subclass. These findings are in line with the notion that HNF4A induces hepatocyte differentiation of cancer stem cells, suppressing hepatocyte proliferation and oncogenesis. $(30,32,33)$

\section{Periportal-type HCCs show the most favorable clinical features and the highest early (2-year) disease-free and overall survival rates after resection.}

Comparative analysis of the clinical outcome of the four HCC subclasses in a 247HCC transcriptomic dataset(34) (dataset C, Supporting Table S2) revealed increasingly aggressive tumor phenotypes from Periportal through Perivenous $\rightarrow$ ECM $\rightarrow$ STEM HCCs (Fig. 6A). These subclasses showed a progressive increase in metastatic signature(34) rates, CLIP and BCLC scores as well as serum AFP concentrations. Survival curves (Fig. 6B) showed a progressive decrease in overall and disease free survival (DF) rates from Periportal through Perivenous $\rightarrow$ ECM $\rightarrow$ STEM HCCs. Thus, the Periportal-type is a well-differentiated, favorable-outcome HCC subclass carrying wild-type CTNNB1, displaying a periportal liver metabolic program and expressing HNF4A target genes. In turn, the Perivenous-type is a welldifferentiated HCC subclass carrying mutant CTNNB1, displaying a perivenous liver metabolic program and expressing $\beta$-catenin target genes. 
The above data were further validated using an external 210-HCC RNAseq dataset. Tumor aggressiveness was inferred from increasing AFP serum levels, tumor grade, TNM staging, vascular invasion, TP53 mutation rates, tumor onset in younger patients (Fig. 6C), as well as survival (Supporting Fig. S13). This analysis confirmed the existence of increasingly aggressive tumor phenotypes from Periportal-type through Perivenous-type $\rightarrow$ ECM-type $\rightarrow$ STEM-type HCCs. The overall survival of Perivenous-type HCCs was significantly more favorable than that of STEM-type HCCs in both the 247-HCC transcriptomic (Fig. 6B) and in the 210-HCC RNAseq (Supporting Fig. S13) datasets.

To obtain a Periportal-type HCC gene signature, we searched for genes meeting the following criteria: $>2$ fold change between the Periportal and the other HCC subclasses; high connectivity within the periportal gene network in HCCs $(>0.30$ correlation coefficient with $>50 \%$ of the genes $)$ and association with survival $(p<0.05$ in $\geq 7 / 8$ tests; Supporting Materials and Methods). The resulting 8-gene Periportaltype HCC signature (Fig. 6D) was associated with favorable overall and disease-free outcome in the 247-HCC transcriptomic (34) (Fig. 6E) and in the 210-HCC RNAseq (Fig. 6F) datasets. Periportal-type HCCs showed the lowest early (2-year) recurrence and the highest overall survival rates after resection among all other HCCs taken together. Univariate and multivariate survival analysis revealed that the Periportaltype HCC signature was independently associated with low early recurrence after HCC resection (Table 1).

Of note, the Periportal-type HCC signature was predominantly expressed in normal liver, as shown by analysis of their mRNA expression levels in 84 tissues, $(35,36)$ from the GSE1133 microarray dataset (Supporting Fig. S14,A-H). In addition, the expression levels of the eight genes constituting the signature were highly correlated 
(Supporting Table S12). These genes were expressed at higher levels in Periportaltype HCCs than in the other HCC subclasses (Fig. 6D). The expression levels of these genes in 70 Periportal HCCs were closer to those detected in 232 non-tumor livers than in 167 non-Periportal HCCs. Two of these genes (AGXT and OTC) were not significantly different in non-tumor livers from Periportal HCCs (Supporting Fig. S15).
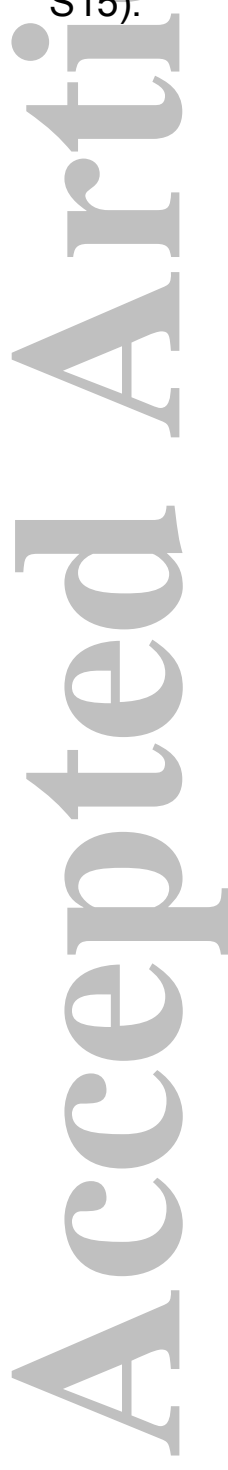


\section{DISCUSSION}

Our study revealed that preservation of the liver zonation program is an important feature of well-differentiated HCCs. Periportal-type HCCs showed the most favorable patient outcome in two independent cohorts of 247 and 210 patients. Further, they displayed the lowest rates of Metastasis Signature,(34) BCLC, CLIP, TNM staging, vascular invasion and TP53 mutations. We identified an 8-gene Periportal-type HCC signature composed of liver-enriched genes involved in periportal liver functions, such as amino-acid catabolism (GLS2, AGXT and GNMT), biliary acid transport (SLC10A1 and SCL22A7), lipid (SLC27A5) and glucose (FETUB) metabolism, as

well as the urea cycle (OTC). These genes were expressed at higher levels in nontumor livers and in Periportal-type than in non-Periportal-type HCCs.

In normal liver, $\beta$-catenin is transiently activated in perivenous zones to meet homeostatic needs.(15) Therefore, turning OFF $\beta$-catenin in adult mouse liver constitutively leads to a default periportal metabolic program, with a normal liver function. Conversely, turning $O N \quad \beta$-catenin leads to liver hyperplasia, hyperammoniemia and death.(20) Considered in this context, Periportal-type HCCs preserve the default metabolic program of normal liver. Therefore, converging evidence indicates that the periportal program embodies the lowest level of aggressiveness in HCCs.

Recently, no association was detected between $\beta$-catenin mutations and survival in 362 HCC patients,(7) in line with data summarized in Supporting Table S1. We confirmed these findings by analyzing two independent cohorts of 247 and $210 \mathrm{HCC}$ patients (Fig. 7A). Noteworthy, subclass-specific survival analyses in these two cohorts revealed that Periportal-type and Perivenous-type HCC patients had a more favorable outcome than STEM-type HCC patients (Fig. 6B; Supporting Fig. S13). 
Also, discriminant analysis confirmed that Perivenous-type HCCs lie between Periportal-type and ECM-type HCCs in terms of aggressiveness (Fig. 7B). This body of evidence suggests that HCCs carrying $\beta$-catenin mutations are moderately aggressive (Fig. 7C). De novo expression of cancer stem/progenitor cell genes such as ODAM and LGR5 in CTNNB1-mutated HCCs goes well with the moderate aggressiveness of this tumor subclass. ODAM is a basement membrane component of the periodontal junctional epithelium secreted by ameloblasts, repressed in adult tissues, but expressed de novo in cancers.(37) LGR5, in turn, is expressed by liver stem cells or progenitors in response to stimuli calling for tissue renewal or tumor cell growth.(38)

Perivenous-type HCCs match the G6(6) and CTNNB1(4) subclasses that carry CTNNB1 mutations, but not the $\mathrm{S} 3(5)$ subclass. In turn, the $\mathrm{S} 3$ subclass matches Periportal-type HCCs. Indeed, S3 HCCs are well-differentiated, hepatocyte-like, and approximately $50 \%$ of them carry CTNNB1 mutations.(5) Further, S3 HCC patients have a more favorable survival than S1 and S2 HCC patients.(27) As the main feature of S3 HCCs is preserved hepatocyte differentiation, S3's may include both Periportal-type and Perivenous-type HCCs.

GLUL, HAL and VNN1 are involved in metabolic liver zonation. $H A L$ is an enzyme that degrades the amino acid histidine(39) and we showed that it was restricted to periportal hepatocytes in normal liver. Likewise, Periportal-type HCCs expressed a host of amino acid degrading enzymes, such as ARG1 (arginase 1) and GLS2 (glutaminase 2), which were major hubs in the periportal gene network in HCCs (Fig. $5 A$ ). Some of these amino acid degrading enzymes, such as SDS (serine dehydratase) and GLDC (glycine decarboxylase), yield the end-product oxaloacetate, which is used for gluconeogenesis, a periportal metabolic pathway. $(20,40)$ VNN1 is 
a key activator of hepatic gluconeogenesis, transcriptionally activated by HNF4A.(41)

By immunohistochemistry, VNN1 had a panlobular distribution, in consistency with unsupervised transcriptomic data, $(20,40)$ suggesting that VNN1 probably responds to dynamic zonation.(29)

Cross-talks between HNF4A and $\beta$-catenin control the transcription of genes involved in periportal and perivenous zonation in normal liver.(29) Our data suggest that this cross-talk is preserved in well-differentiated HCCs. The 5-gene score predicting CTNNB1 mutation was composed of genes known to be upregulated by $\beta$-catenin (LGR5, GLUL and ODAM) or by HNF4A (VNN1 and HAL).(20, 41) By in vitro experiments, we confirmed that $\beta$-catenin activation enhanced GLUL, LGR5 and ODAM, but inhibited $H A L$ and VNN1 expression. Conversely, $\beta$-catenin knockdown silenced AXIN2 and ODAM, but enhanced HAL and VNN1 expression. Further, we showed that $\beta$-catenin activation attenuated the gain in the expression of $H N F 4 A$, $A L D O B, H A L$ and VNN1 upon the 30-day time course of differentiation of HepaRG progenitors into hepatocyte-like cells (Supporting Fig. S5C-F). These observations suggest that $\beta$-catenin mutations divert the default periportal metabolic program of well-differentiated HCC cells toward a perivenous one (Fig. 7B).

In summary, we identified a new well-differentiated HCC subclass, Periportal-type HCCs, which has the most favorable clinical outcome among all HCCs. Accordingly, the Periportal-type HCC signature was associated with low early HCC recurrence (< 2 years after resection) independently of tumor size and vascular invasion in multivariate analyses. Given the heterogeneity of HCC phenotypes shown in Fig 7B, the question arises whether early, well-differentiated HCC types (Periportal and Perivenous) ultimately progress to a more aggressive, progenitor-like phenotype spontaneously or in response to treatments that may spare cancer progenitor cells. 
Prediction of patient survival impacts the indication of liver transplantation for HCC. In particular, early recurrence is one of the principles governing patient staging within the spectrum of eligibility for liver transplantation.(3) Thus, it would be interesting to test whether identification of Periportal-type HCCs contributes to adapt indications of liver transplantation beyond current criteria. This issue will gain importance in the near future, because the control of the hepatitis $C$ virus epidemics with direct antiviral agents will increase donor liver availability.

The major limitation to the direct translation of these basic findings to the clinical arena is the exclusion of diagnostic liver biopsy from current guidelines for HCC diagnosis and allocation to treatments. However, in view of exciting results demonstrating the clinical relevance of metabolic imaging in early-stage $\operatorname{HCCs}(42)$ and of the association of the transporter of gadoxetic acid OATP1B3 with Wnt/Bcatenin signaling in $\mathrm{HCCs}(43)$, the Periportal-type molecular phenotype raises a new challenge to metabolic imaging of HCCs.

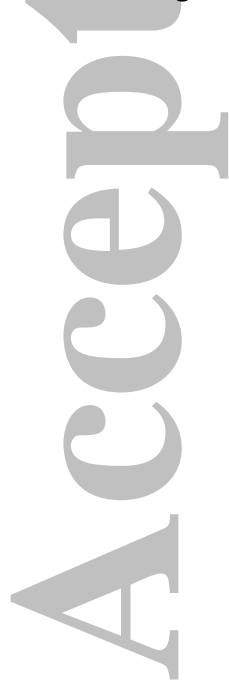




\section{FIGURE LEGENDS}

Fig. 1. Flowchart of the study. (A) Construction of a transcriptomic metadata set for subclass-specific analyses after curative resection of 1133 HCCs. Nine public transcriptomic datasets ( $A$ to $I)$ were merged into a metadata set. As the 1133-HCCs were not annotated for CTNNB1 mutations, we developed a CTNNB1 mutation prediction score from transcriptomic data. The most stable markers were identified by SPLS-DA modeling in training and validation sets. Mutation prediction was validated in an external in-house HCC collection(9); then, CTNNB1 mutations were predicted in 1133 HCCs after cross-platform normalization. Subclass-specific survival analyses were performed in dataset $\mathrm{C}(\mathrm{n}=247 \mathrm{HCCs})(34)$ and validated in an external RNAseq, full-genome sequenced, dataset (LIHC-US; TCGA consortium, $n=210$ HCCs).(10) (B) Transcriptomic datasets used: 1, yes or present; 0, no or absent. References: Training set(4); Validation set(6); $A(44) ; B(45) ; C(34) ; D(46) ; E(47)$; $F(48) ; G(49) ; H(49) ; I(28) ;$ RNAseq dataset(10). Major endpoints are indicated by bold arrows. External validation sets are represented by black boxes.

Fig. 2. A 5-gene score reliably predicts CTNNB1 mutations using mRNA expression data in HCCs. (A) Gene expression of the five biomarkers in the training $(n=87)$, validation $(n=56)$ and the external validation $(n=82)$ sets, according to Sangersequenced CTNNB1 mutational status. AXIN2 was included as a Wnt pathway activation marker (missing in A2). (B) Heatmaps showing mRNA expression of the five biomarkers plus $A X I N 2$ and CTNNB1 mutational status (B1, training set; B2, validation set; B3, external HCC collection). Low and high gene expressions (median as cut-off) are represented in green and red, respectively. (C) Formula; (D) 
Performance and (E) ROC curves of the CTNNB1 mutation prediction score. (F) Tissue micro array-based immunostainings (brown) for GLUL, HAL and VNN1 in 20 CTNNB1 mutant and 20 CTNNB1 wild-type HCCs. Insets on the right: $\beta$-catenin cell membrane staining in CTNNB1 wild-type (arrowheads, upper right), or cytoplasmic and nuclear staining in CTNNB1 mutant HCCs (arrowheads, lower right). (G) Immunostaining (brown) for ODAM and GLUL in serial sections in a CTNNB1 mutant HCC. Images were acquired with a tissue slide scanner at 20X original magnification. ${ }^{*} \mathrm{P}<0.05,{ }^{* *} \mathrm{P}<0.01,{ }^{* * *} \mathrm{P}<0.001$ (Wilcoxon tests). AUROC, Area under the receiver operating curve; qPCR, real-time PCR (a pool of five normal liver samples was used as calibrator); CI, Confidence interval; Se, Sensitivity; Sp, Specificity.

Fig. 3. (A) Hierarchical clustering of transcriptomic metadata from 1133 HCCs reveals four tumor subgroups (green, red, purple, \& blue) with different proportions of predicted CTNNB1 mutations. Lowest and highest gene expression values are represented in green and red, respectively. (B) Gene expression levels of the five CTNNB1 mutation predictors. Results are relative to the green subclass, arbitrarily set to $1 \pm 95 \% \mathrm{Cl}$. Green, $n=326$; Red, $n=210$; Blue, $n=420$; Purple, $n=177$. NS, nonsignificant. ${ }^{* *} \mathrm{P}<0.01,{ }^{* * *} \mathrm{P}<0.001$, after Student's $t$ tests. (C) Immunostainings (brown) of $\beta$-catenin, GLUL, VNN1 and HAL in serial sections of normal human liver illustrates clear-cut perivenous and periportal zonation of GLUL and HAL, respectively. $\beta$-catenin is predominantly membranous and highlights bile ducts $(B D)$. Arrows show portal tracts $(P T)$ and central veins $(C V)$. VNN1 is panlobular. (D) VNN1 is detected in bile canaliculi in normal liver and in a well-differentiated HCC. VNN1 signal is totally suppressed by the immunogen polypeptide. 
Fig. 4. Well-differentiated HCCs display a preserved metabolic liver zonation program. (A) Gene Set Enrichment Analysis (GSEA) in the four HCC subclasses. (B) Expression of relevant genes in the 1133-HCC metadata set. Color code keys are shown at the bottom of the heatmap. Samples (columns) are ordered by HCC subclass. Green, low; red, high expression. PP, Periportal-type; PV, Perivenous-type; ECM, Extracellular matrix-type; STEM, Stem cell-type.

Fig. 5. The metabolic program of Periportal-type HCCs is regulated by HNF4A. (A) Periportal (PP, green) and Perivenous ( $P V$, red) gene networks constructed by Weighted Gene Correlation Analysis and shown with Cytoscape graphics. Networks consist of human orthologs of mouse liver periportal and perivenous gene signatures,(20) detected in 326 Periportal-type and 210 Perivenous-type HCCs from the 1133 HCC set. Node border colors (Green/red) denote periportal/perivenous attributes of mouse genes genes, respectively.(20) Node core colors are proportional to the PV/PP fold-change in the HCC set (Color key: lower right). Link thickness is proportional to correlation coefficients ( $>0.30$ in all networks; blue, positive; red, negative correlation). (B) The 550 genes representative of the four HCC subclasses discriminate between Hnf4a-KO and Hnf4a-WT mice.(31). Three gene clusters (black nodes on the left) denote: 1, upregulation in Hnf4a-KO's and enrichment in human HCC STEM/ECM signatures; 2, upregulation in Hnf4a-WT's and enrichment in HCC PP genes; 3, genes poorly affected by Hnf4a status. Red, high expression, green, low expression. 
Fig. 6. Periportal-type HCCs show the most favorable clinical features and the highest early (2-year) disease-free and overall survival rates after resection. (A) Clinical features of HCC subclasses in a 247-patient dataset(34). (B) Kaplan-Meier plots of subclass-specific overall and disease-free survival; ${ }^{*} \mathrm{P}<0.05,{ }^{* *} \mathrm{P}<0.01$, ${ }^{* * *} P<0.001$. (C) Subclass-specific clinical features, CTNNB1 and TP53 mutation rates (full genome sequencing) in an external validation 210-HCC RNAseq dataset (TGCA-LIHC-US). (D) Subclass-specific mRNA expression levels of Periportal HCC signature genes in 1133 HCCs. (E, F) Kaplan-Meier plots of overall and disease-free survival in HCC patients with and without the Periportal HCC signature in two datasets. AFP, serum alpha-fetoprotein; NA, not available; BCLC (Barcelona Clinic Liver Cancer); CLIP (Cancer of the Liver Italian Program). Statistics: Fisher exact test (categorical variables); Student's $t$ test (continuous variables); Log-rank test (survival analyses).

Fig. 7. Periportal-type HCCs and the survival paradox between CTNNB1-mutated and wild-type HCCs. (A) Kaplan-Meier plots and log-rank tests in two independent HCC datasets confirm similar outcome of HCCs carrying mutated (red) or wild-type (black) CTNNB1. Mutational status results from prediction in dataset $\mathrm{C}$ and fullgenome sequencing in TCGA-LICH-US. (B) Multivariate discriminant analysis of the four HCC subclasses. In the first component, there is a grading from Periportal-type (green), through Perivenous- (red) and ECM-types (blue), to STEM-type (purple). In the second component, Perivenous-type differs from other HCC subclasses. (C) HCCs with predominantly mutated CTNNB1 are moderately aggressive. (D) HCCs with predominantly wild-type CTNNB1 include mildly, moderately and highly aggressive subclasses. The \% of HCCs in each subclass is indicated in parentheses. 
Acknowledgements: We thank the following core facilities: «Structure Fédérative de Recherche en Biologie et Santé de Rennes » UMS CNRS 3480/US INSERM 018 (Health Genomics, Jean Moser; High Precision Histopathology, Alain Fautrel); SynNanoVect electroporation facility (Pascal Loyer). We are indebted to Patricia Jouas and Michèle Le Guennec for secretarial support, to Edouard Curran for CTNNB1 deletion analysis, to Sihem Mebarki for mRNA extraction, to Gaelle Angenard and Catherine Ribault for tissue banking. We thank Marie-Jo Brion (The University of Queensland Diamantina Institute, the University of Queensland) for her thorough editing contribution. We thank Laurent Sulpice and Damien Bergeat (Dept of Gastrointestinal and Hepatobiliary Surgery, Rennes, France) for helpful discussion.

Financial support: Inserm, Univ Rennes 1, Région Bretagne, Agence Nationale de la Recherche, Institut National du Cancer, Fondation Recherche Médicale, Ligue Nationale Contre le Cancer (Comité des Côtes d'Armor), Feder, Contrat de Plan Etat Région 2007-2013, projet Cancéropôle.

Contributors: Study design, RD, OM, FR, K-A LC, CP, BC; statistics, RD, FR, K-A LC; experiments, RD, FC, MS, SR, PB, CP, OM; anatomic pathology data, BT, OM; tissue banking and quality control, MD, OM; manuscript preparation, $\mathrm{RD}, \mathrm{OM}$; manuscript editing, all authors.

Competing interests: None 
Sample collection: Sample collection was reported to the Ministry of Education and Research (No. DC-2008-338).

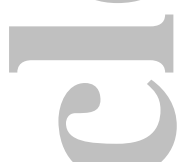

Ethics approval: The study protocol complied with French laws and regulations and was approved by INSERM's Institutional Review Board (number 01-036) in the context of the National Network of Liver Biological Resource Centers.
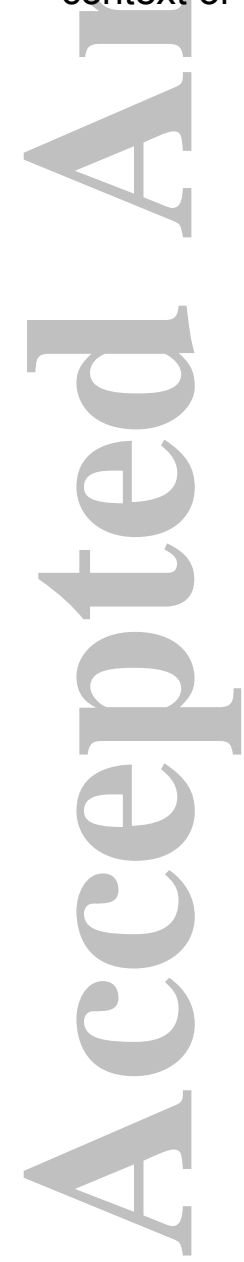


\section{REFERENCES}

1. Llovet JM, Zucman-Rossi J, Pikarsky E, Sangro B, Schwartz M, Sherman M, Gores G. Hepatocellular carcinoma. Nature reviews. Disease primers 2016;2:16018.

2. Zucman-Rossi J, Villanueva A, Nault JC, Llovet JM. Genetic Landscape and Biomarkers of Hepatocellular Carcinoma. Gastroenterology 2015;149:1226-1239 e1224.

3. Sapisochin G, Bruix J. Liver transplantation for hepatocellular carcinoma: outcomes and novel surgical approaches. Nature reviews. Gastroenterology \& hepatology 2017;14:203-217.

4. Chiang DY, Villanueva A, Hoshida Y, Peix J, Newell P, Minguez B, LeBlanc AC, et al. Focal gains of VEGFA and molecular classification of hepatocellular carcinoma. Cancer research 2008;68:6779-6788.

5. Hoshida Y, Nijman SM, Kobayashi M, Chan JA, Brunet JP, Chiang DY, Villanueva A, et al. Integrative transcriptome analysis reveals common molecular subclasses of human hepatocellular carcinoma. Cancer research 2009;69:73857392.

6. Boyault S, Rickman DS, de Reynies A, Balabaud C, Rebouissou S, Jeannot E, Herault A, et al. Transcriptome classification of HCC is related to gene alterations and to new therapeutic targets. Hepatology 2007;45:42-52.

7. Rebouissou S, Franconi A, Calderaro J, Letouze E, Imbeaud S, Pilati C, Nault JC, et al. Genotype-phenotype correlation of CTNNB1 mutations reveals different sscatenin activity associated with liver tumor progression. Hepatology 2016.

8. Lachenmayer A, Alsinet C, Savic R, Cabellos L, Toffanin S, Hoshida Y, Villanueva A, et al. Wnt-pathway activation in two molecular classes of hepatocellular 
carcinoma and experimental modulation by sorafenib. Clinical cancer research : an official journal of the American Association for Cancer Research 2012;18:4997-5007. 9. Mebarki S, Desert R, Sulpice L, Sicard M, Desille M, Canal F, Schneider HD, et al. De novo HAPLN1 expression hallmarks Wnt-induced stem cell and fibrogenic networks leading to aggressive human hepatocellular carcinomas. Oncotarget 2016. 10. Aran D, Sirota M, Butte AJ. Systematic pan-cancer analysis of tumour purity. Nature communications 2015;6:8971.

11. Leek JT, Johnson WE, Parker HS, Jaffe AE, Storey JD. The sva package for removing batch effects and other unwanted variation in high-throughput experiments.

Bioinformatics 2012;28:882-883.

12. Le Cao KA, Rohart F, McHugh L, Korn O, Wells CA. YuGene: a simple approach to scale gene expression data derived from different platforms for integrated analyses. Genomics 2014;103:239-251.

13. Rohart F, Mason EA, Matigian N, Mosbergen R, Korn O, Chen T, Butcher S, et al. A molecular classification of human mesenchymal stromal cells. PeerJ 2016;4:e1845.

14. Zucman-Rossi J, Benhamouche S, Godard C, Boyault S, Grimber G, Balabaud C, Cunha AS, et al. Differential effects of inactivated Axin1 and activated beta-catenin mutations in human hepatocellular carcinomas. Oncogene 2007;26:774780.

15. Wang B, Zhao L, Fish M, Logan CY, Nusse R. Self-renewing diploid Axin2(+) cells fuel homeostatic renewal of the liver. Nature 2015;524:180-185.

16. Guichard C, Amaddeo G, Imbeaud S, Ladeiro Y, Pelletier L, Maad IB, Calderaro $\mathrm{J}$, et al. Integrated analysis of somatic mutations and focal copy-number 
changes identifies key genes and pathways in hepatocellular carcinoma. Nature genetics 2012;44:694-698.

17. Sato N, Meijer L, Skaltsounis L, Greengard P, Brivanlou AH. Maintenance of pluripotency in human and mouse embryonic stem cells through activation of Wnt signaling by a pharmacological GSK-3-specific inhibitor. Nature medicine $2004 ; 10: 55-63$.

18. Farre D, Roset R, Huerta M, Adsuara JE, Rosello L, Alba MM, Messeguer X. Identification of patterns in biological sequences at the ALGGEN server: PROMO and MALGEN. Nucleic acids research 2003;31:3651-3653.

19. Benhamouche S, Decaens T, Godard C, Chambrey R, Rickman DS, Moinard C, Vasseur-Cognet M, et al. Apc tumor suppressor gene is the "zonation-keeper" of mouse liver. Dev Cell 2006;10:759-770.

20. Gougelet A, Torre C, Veber P, Sartor C, Bachelot L, Denechaud PD, Godard C, et al. T-cell factor 4 and beta-catenin chromatin occupancies pattern zonal liver metabolism in mice. Hepatology 2014;59:2344-2357.

21. Kristiansen TZ, Bunkenborg J, Gronborg M, Molina H, Thuluvath PJ, Argani P, Goggins MG, et al. A proteomic analysis of human bile. Molecular \& cellular proteomics : MCP 2004;3:715-728.

22. Hoshida Y, Villanueva A, Kobayashi M, Peix J, Chiang DY, Camargo A, Gupta $\mathrm{S}$, et al. Gene expression in fixed tissues and outcome in hepatocellular carcinoma. The New England journal of medicine 2008;359:1995-2004.

23. Lee JS, Chu IS, Heo J, Calvisi DF, Sun Z, Roskams T, Durnez A, et al. Classification and prediction of survival in hepatocellular carcinoma by gene expression profiling. Hepatology 2004;40:667-676. 
24. Naba A, Clauser KR, Hoersch S, Liu H, Carr SA, Hynes RO. The matrisome: in silico definition and in vivo characterization by proteomics of normal and tumor extracellular matrices. Molecular \& cellular proteomics : MCP 2012;11:M111 014647. 25. Wong DJ, Liu H, Ridky TW, Cassarino D, Segal E, Chang HY. Module map of stem cell genes guides creation of epithelial cancer stem cells. Cell stem cell 2008;2:333-344.

26. Yamashita T, Ji J, Budhu A, Forgues M, Yang W, Wang HY, Jia H, et al. EpCAM-positive hepatocellular carcinoma cells are tumor-initiating cells with stem/progenitor cell features. Gastroenterology 2009;136:1012-1024.

27. Kan Z, Zheng H, Liu X, Li S, Barber TD, Gong Z, Gao H, et al. Whole-genome sequencing identifies recurrent mutations in hepatocellular carcinoma. Genome research 2013;23:1422-1433.

28. Woo HG, Park ES, Cheon JH, Kim JH, Lee JS, Park BJ, Kim W, et al. Gene expression-based recurrence prediction of hepatitis B virus-related human hepatocellular carcinoma. Clinical cancer research : an official journal of the American Association for Cancer Research 2008;14:2056-2064.

29. Berasain C, Avila MA. Deciphering liver zonation: new insights into the betacatenin, Tcf4, and HNF4alpha triad. Hepatology 2014;59:2080-2082.

30. Bonzo JA, Ferry CH, Matsubara T, Kim JH, Gonzalez FJ. Suppression of hepatocyte proliferation by hepatocyte nuclear factor 4 alpha in adult mice. The Journal of biological chemistry 2012;287:7345-7356.

31. Holloway MG, Miles GD, Dombkowski AA, Waxman DJ. Liver-specific hepatocyte nuclear factor-4alpha deficiency: greater impact on gene expression in male than in female mouse liver. Molecular endocrinology 2008;22:1274-1286. 
32. Ning BF, Ding J, Yin C, Zhong W, Wu K, Zeng X, Yang W, et al. Hepatocyte nuclear factor 4 alpha suppresses the development of hepatocellular carcinoma. Cancer research 2010;70:7640-7651.

33. Hatziapostolou M, Polytarchou C, Aggelidou E, Drakaki A, Poultsides GA, Jaeger SA, Ogata $\mathrm{H}$, et al. An HNF4alpha-miRNA inflammatory feedback circuit regulates hepatocellular oncogenesis. Cell 2011;147:1233-1247.

34. Roessler S, Jia HL, Budhu A, Forgues M, Ye QH, Lee JS, Thorgeirsson SS, et al. A unique metastasis gene signature enables prediction of tumor relapse in earlystage hepatocellular carcinoma patients. Cancer research 2010;70:10202-10212.

35. Su Al, Wiltshire T, Batalov S, Lapp H, Ching KA, Block D, Zhang J, et al. A gene atlas of the mouse and human protein-encoding transcriptomes. Proceedings of the National Academy of Sciences of the United States of America 2004;101:60626067.

36. Wu C, Jin X, Tsueng G, Afrasiabi C, Su Al. BioGPS: building your own mashup of gene annotations and expression profiles. Nucleic acids research 2016;44:D313-316.

37. Foster JS, Fish LM, Phipps JE, Bruker CT, Lewis JM, Bell JL, Solomon A, et al. Odontogenic ameloblast-associated protein (ODAM) inhibits growth and migration of human melanoma cells and elicits PTEN elevation and inactivation of PI3K/AKT signaling. BMC cancer 2013;13:227.

38. Mebarki S, Desert R, Sulpice L, Sicard M, Desille M, Canal F, Dubois-Pot Schneider $\mathrm{H}$, et al. De novo HAPLN1 expression hallmarks Wnt-induced stem cell and fibrogenic networks leading to aggressive human hepatocellular carcinomas. Oncotarget 2016;7:39026-39043. 
39. Aleman G, Ortiz V, Contreras AV, Quiroz G, Ordaz-Nava G, Langley E, Torres $\mathrm{N}$, et al. Hepatic amino acid-degrading enzyme expression is downregulated by natural and synthetic ligands of PPARalpha in rats. The Journal of nutrition 2013;143:1211-1218.

40. Braeuning A, Ittrich C, Kohle C, Hailfinger S, Bonin M, Buchmann A, Schwarz M. Differential gene expression in periportal and perivenous mouse hepatocytes. The FEBS journal 2006;273:5051-5061.

41. Chen S, Zhang W, Tang C, Tang X, Liu L, Liu C. Vanin-1 is a key activator for hepatic gluconeogenesis. Diabetes 2014;63:2073-2085.

42. Yamashita T, Kitao A, Matsui O, Hayashi T, Nio K, Kondo M, Ohno N, et al. Gd-EOB-DTPA-enhanced magnetic resonance imaging and alpha-fetoprotein predict prognosis of early-stage hepatocellular carcinoma. Hepatology 2014;60:1674-1685. 43. Ueno A, Masugi Y, Yamazaki K, Komuta M, Effendi K, Tanami Y, Tsujikawa H, et al. OATP1B3 expression is strongly associated with $\mathrm{Wnt} /$ beta-catenin signalling and represents the transporter of gadoxetic acid in hepatocellular carcinoma. Journal of hepatology 2014;61:1080-1087.

44. Kim SM, Leem SH, Chu IS, Park YY, Kim SC, Kim SB, Park ES, et al. Sixtyfive gene-based risk score classifier predicts overall survival in hepatocellular carcinoma. Hepatology 2012;55:1443-1452.

45. Minguez B, Hoshida Y, Villanueva A, Toffanin S, Cabellos L, Thung S, Mandeli J, et al. Gene-expression signature of vascular invasion in hepatocellular carcinoma. Journal of hepatology 2011;55:1325-1331.

46. Sung WK, Zheng H, Li S, Chen R, Liu X, Li Y, Lee NP, et al. Genome-wide survey of recurrent HBV integration in hepatocellular carcinoma. Nature genetics 2012;44:765-769. 
47. Tsuchiya M, Parker JS, Kono H, Matsuda M, Fujii H, Rusyn I. Gene expression in nontumoral liver tissue and recurrence-free survival in hepatitis $C$ viruspositive hepatocellular carcinoma. Molecular cancer 2010;9:74.

48. Villa E, Critelli R, Lei B, Marzocchi G, Camma C, Giannelli G, Pontisso P, et al. Neoangiogenesis-related genes are hallmarks of fast-growing hepatocellular carcinomas and worst survival. Results from a prospective study. Gut 2016;65:861869.

49. Villanueva A, Hoshida Y, Battiston C, Tovar V, Sia D, Alsinet C, Cornella H, et al. Combining clinical, pathology, and gene expression data to predict recurrence of hepatocellular carcinoma. Gastroenterology 2011;140:1501-1512 e1502.

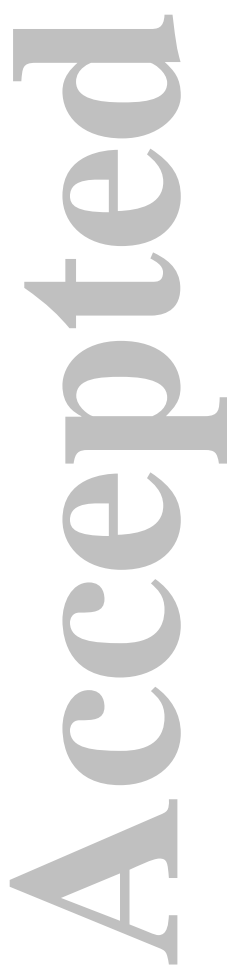




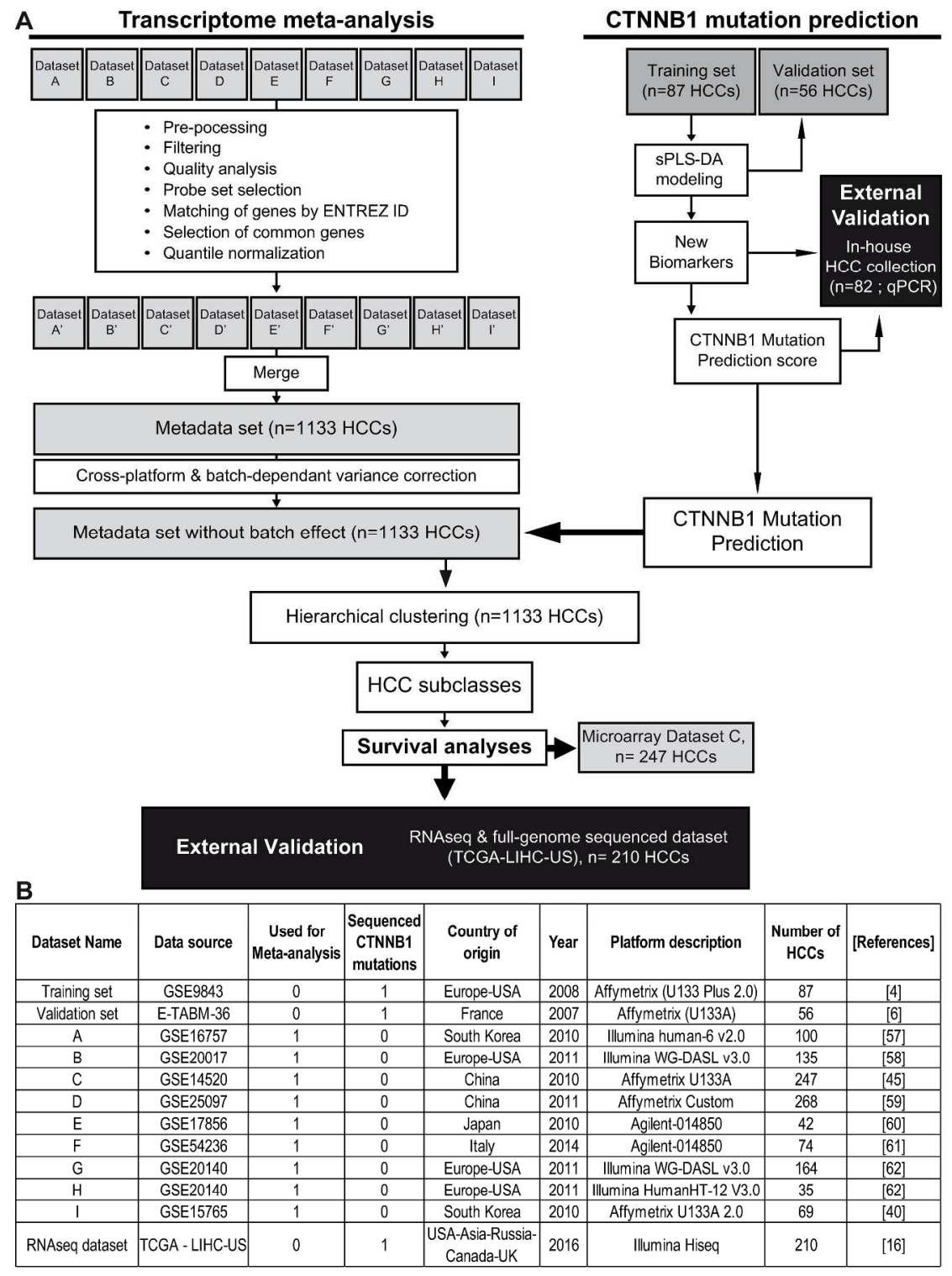

Désert et al., Figure 1

Fig. 1. Flowchart of the study. (A) Construction of a transcriptomic metadata set for subclass-specific analyses after curative resection of 1133 HCCs. Nine public transcriptomic datasets (A to I) were merged into a metadata set. As the 1133-HCCs were not annotated for CTNNB1 mutations, we developed a CTNNB1 mutation prediction score from transcriptomic data. The most stable markers were identified by SPLS-DA modeling in training and validation sets. Mutation prediction was validated in an external in-house HCC collection(9); then, CTNNB1 mutations were predicted in $1133 \mathrm{HCCs}$ after cross-platform normalization. Subclass-specific survival analyses were performed in dataset $C(n=247$ HCCs $)(34)$ and validated in an external RNAseq, full-genome sequenced, dataset (LIHC-US; TCGA consortium, $n=210$ HCCs).(10) (B) Transcriptomic datasets used: 1 , yes or present; 0 , no or absent. References: Training set(3); Validation set(5); $\mathrm{A}(45) ; \mathrm{B}(46) ; \mathrm{C}(34) ; \mathrm{D}(47) ; \mathrm{E}(48) ; \mathrm{F}(49) ; \mathrm{G}(50) ; \mathrm{H}(50) ; \mathrm{I}(28) ;$ RNAseq dataset(10). Major endpoints are indicated by bold arrows. External validation sets are represented by black boxes.

$$
220 \times 311 \mathrm{~mm}(300 \times 300 \text { DPI) }
$$



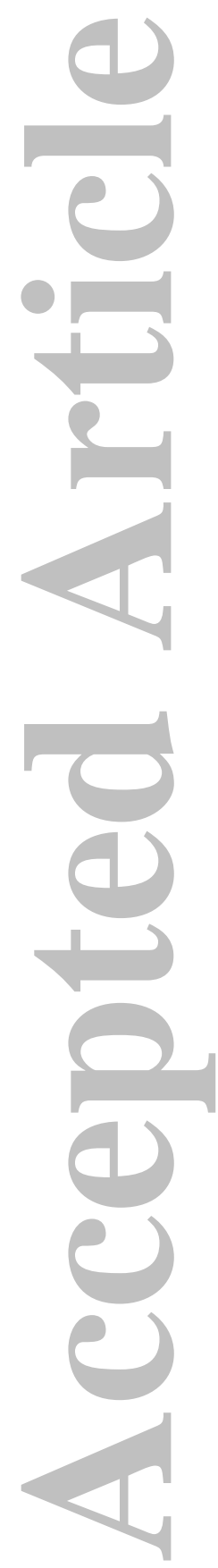

Hepatology

This article is protected by copyright. All rights reserved. 

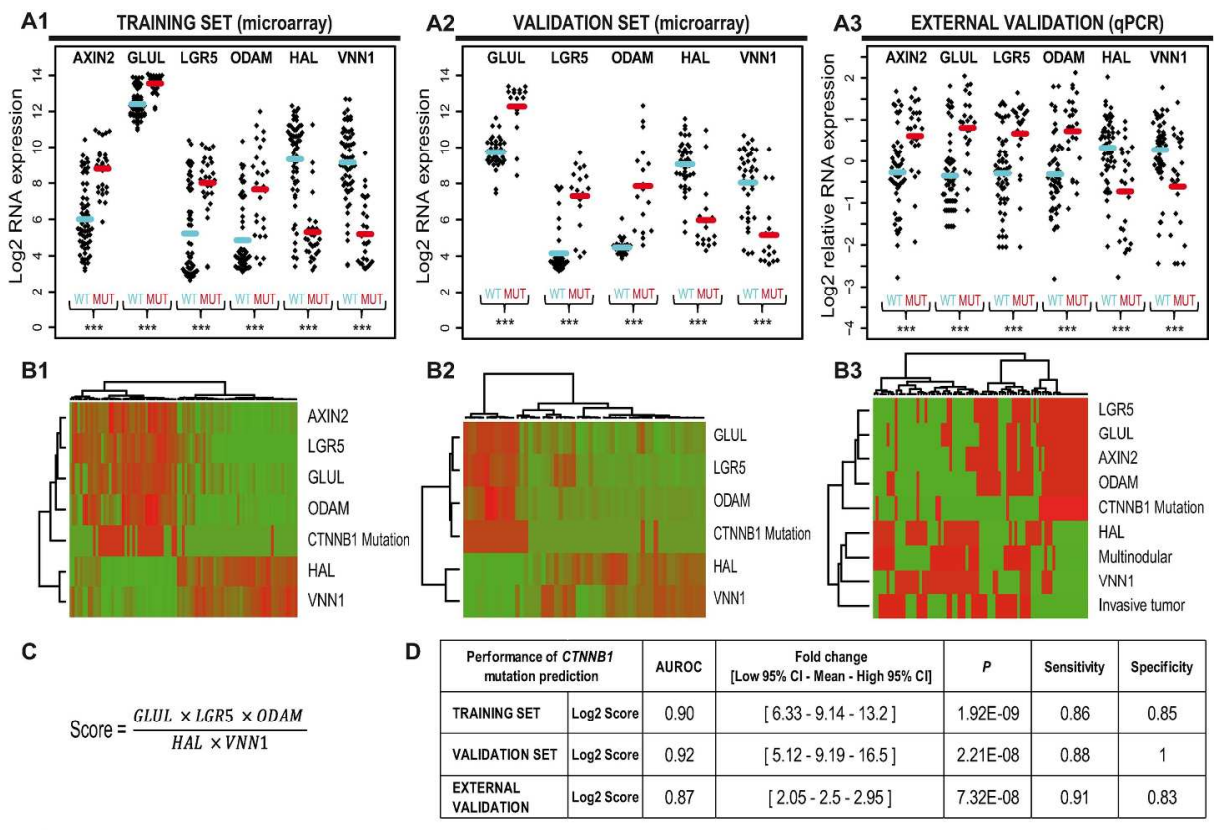

C

\begin{tabular}{|c|c|c|c|c|c|c|}
\hline \multicolumn{2}{|c|}{$\begin{array}{c}\text { Performance of CTNNB1 } \\
\text { mutation prediction }\end{array}$} & \multirow{2}{*}{\begin{tabular}{|c|} 
AUROC \\
0.90
\end{tabular}} & \multirow{2}{*}{$\begin{array}{l}\begin{array}{c}\text { Fold change } \\
\text { [Low 95\% CI- Mean - High 95\% CI] }\end{array} \\
{[6.33-9.14-13.2]}\end{array}$} & \multirow{2}{*}{\begin{tabular}{|c}
$P$ \\
$1.92 E-09$
\end{tabular}} & \multirow{2}{*}{$\begin{array}{c}\text { Sensitivity } \\
0.86\end{array}$} & \multirow{2}{*}{\begin{tabular}{|c|c|} 
Specficitity \\
0.85 \\
\end{tabular}} \\
\hline TRANING SET & Log2 Score & & & & & \\
\hline VALIDATION SET & Log2 Score & 0.92 & {$[5.12-9.19-16.5]$} & $2.21 \mathrm{E}-08$ & 0.88 & 1 \\
\hline $\begin{array}{l}\text { EXTERNAL } \\
\text { VALIDATION }\end{array}$ & |Log2 Score & 0.87 & {$[2.05-2.5-2.95]$} & $7.32 E-08$ & 0.91 & 0.83 \\
\hline
\end{tabular}
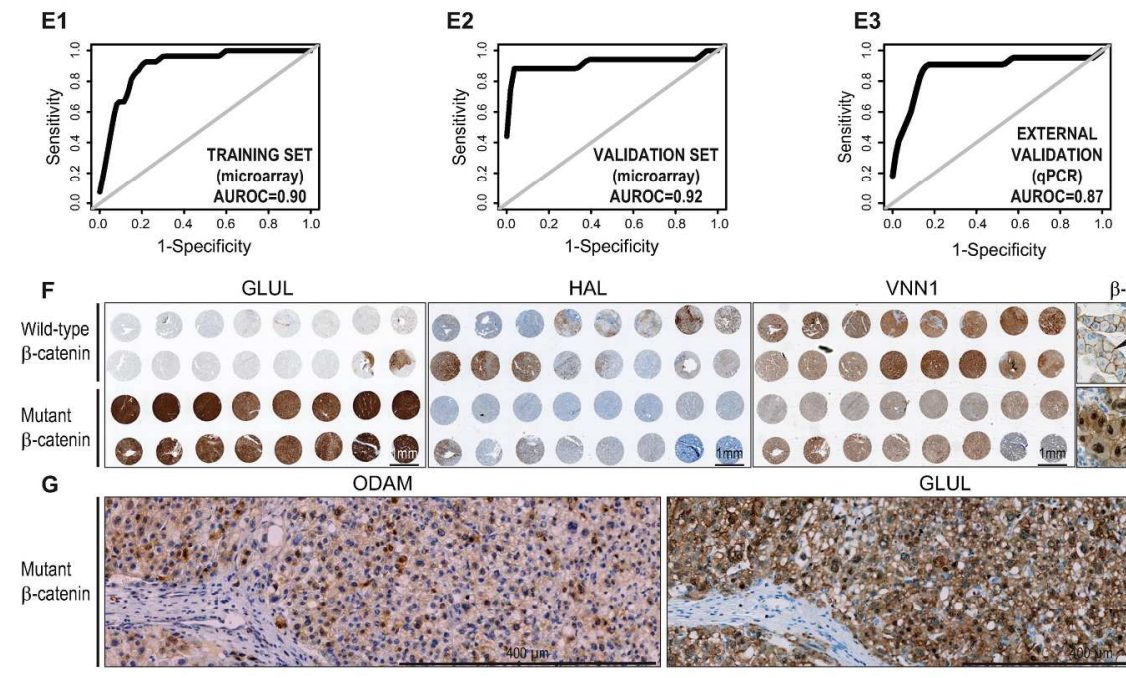

VNN1 $\beta$-catenin

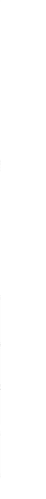


normal liver samples was used as calibrator); CI, Confidence interval; Se, Sensitivity; Sp, Specificity. $219 \times 282 \mathrm{~mm}(300 \times 300 \mathrm{DPI})$
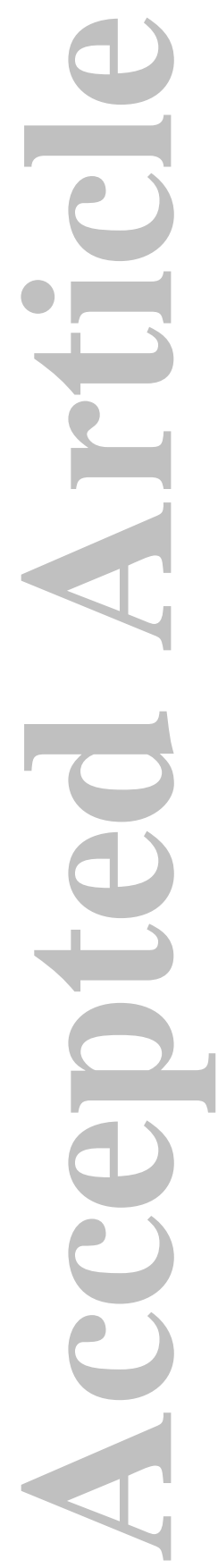

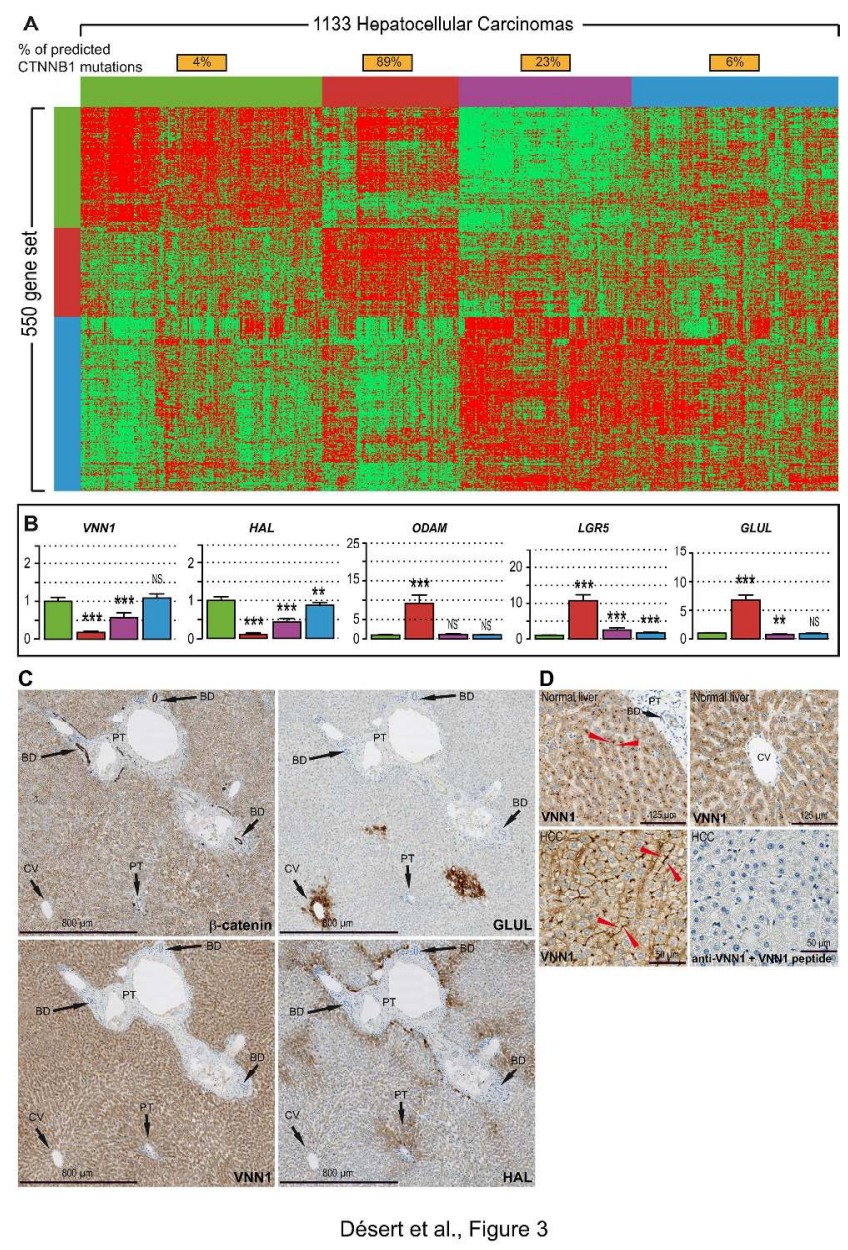

Fig. 3. (A) Hierarchical clustering of transcriptomic metadata from 1133 HCCs reveals four tumor subgroups (green, red, purple, \& blue) with different proportions of predicted CTNNB1 mutations. Lowest and highest gene expression values are represented in green and red, respectively. (B) Gene expression levels of the five CTNNB1 mutation predictors. Results are relative to the green subclass, arbitrarily set to $1 \pm 95 \%$ CI. Green, $n=326$; Red, $n=210$; Blue, $n=420$; Purple, $n=177$. NS, non-significant. $* * p<0.01, * * * P<0.001$, after Student's t tests. (C) Immunostainings (brown) of $\beta$-catenin, GLUL, VNN1 and HAL in serial sections of normal human liver illustrates clear-cut perivenous and periportal zonation of GLUL and HAL, respectively. $\beta$-catenin is predominantly membranous and highlights bile ducts (BD). Arrows show portal tracts (PT) and central veins (CV). VNN1 is panlobular. (D) VNN1 is detected in bile canaliculi in normal liver and in a welldifferentiated HCC. VNN1 signal is totally suppressed by the immunogen polypeptide.

$304 \times 463 \mathrm{~mm}(300 \times 300$ DPI $)$ 

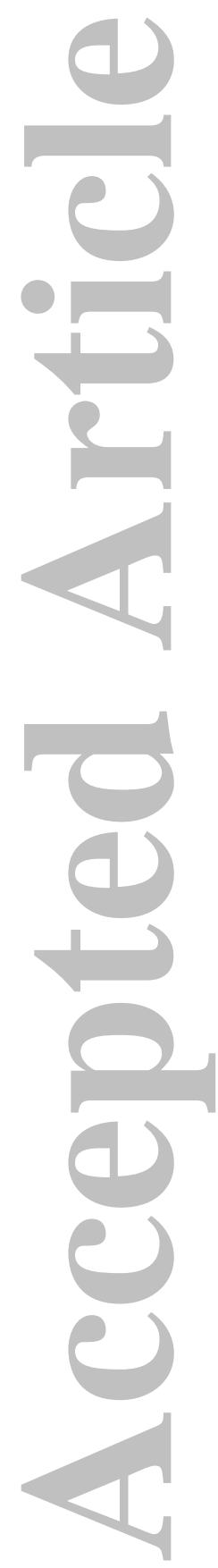

Hepatology

This article is protected by copyright. All rights reserved. 


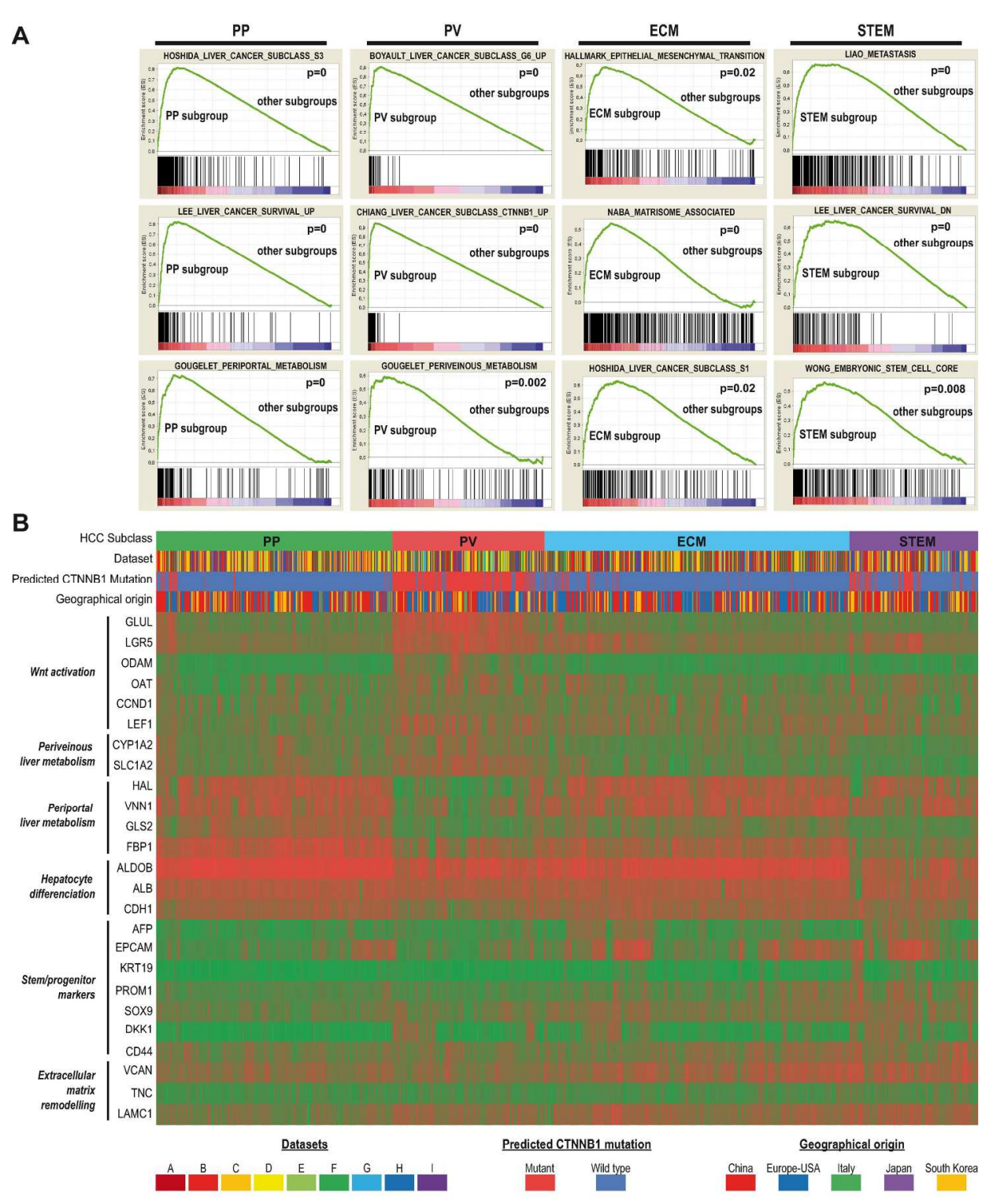

Désert et al., Figure 4

Fig. 4. Well-differentiated HCCs display a preserved metabolic liver zonation program. (A) Gene Set Enrichment Analysis (GSEA) in the four HCC subclasses. (B) Expression of relevant genes in the 1133-HCC metadata set. Color code keys are shown at the bottom of the heatmap. Samples (columns) are ordered by HCC subclass. Green, low; red, high expression. PP, Periportal-type; PV, Perivenous-type; ECM, Extracellular matrix-type; STEM, Stem cell-type.

$212 \times 269 \mathrm{~mm}(300 \times 300$ DPI $)$ 


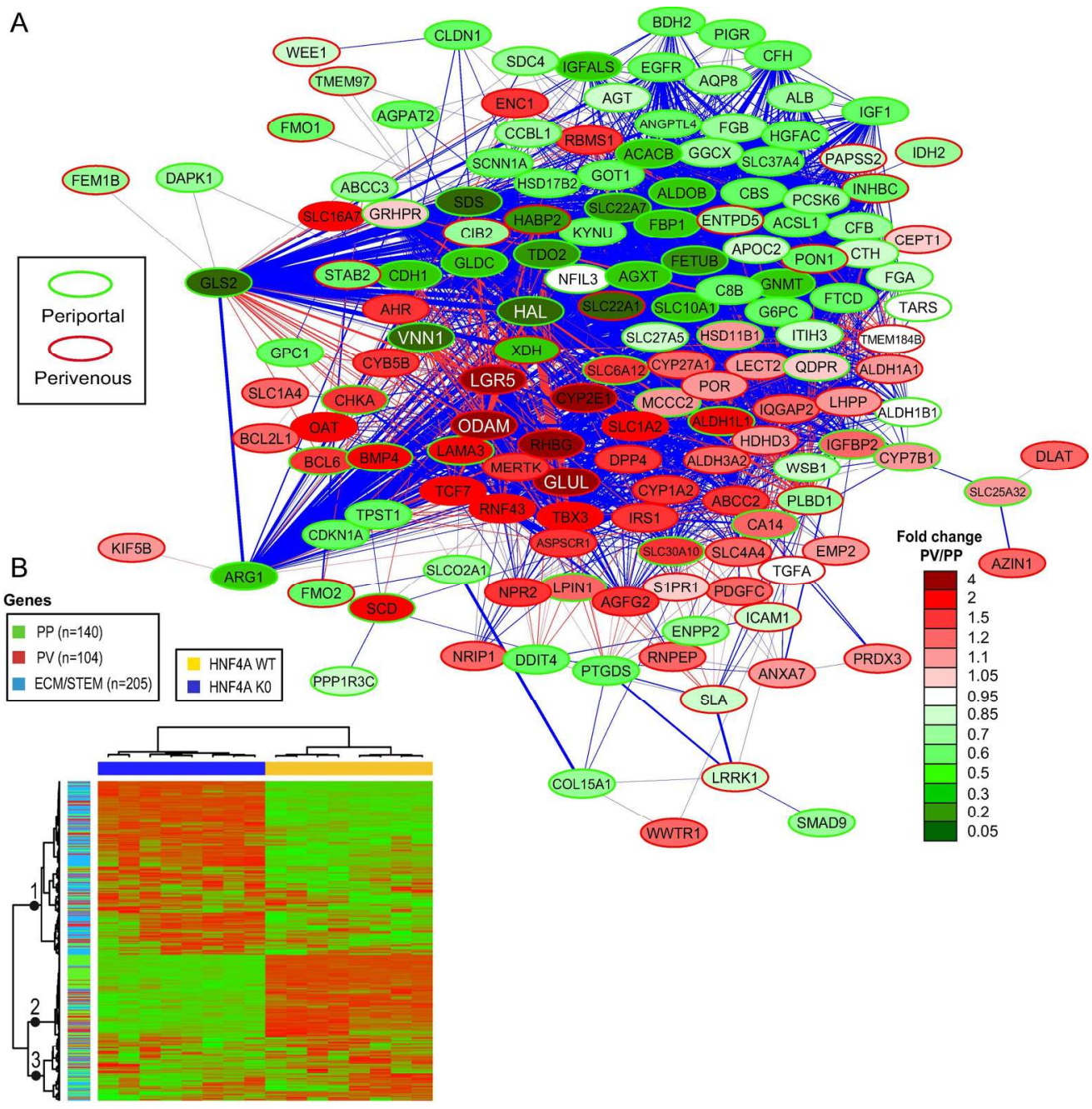

Désert et al., Figure 5

Fig. 5. The metabolic program of Periportal-type HCCs is regulated by HNF4A. (A) Periportal (PP, green) and Perivenous (PV, red) gene networks constructed by Weighted Gene Correlation Analysis and shown with Cytoscape graphics. Networks consist of human orthologs of mouse liver periportal and perivenous gene signatures,(20) detected in 326 Periportal-type and 210 Perivenous-type HCCs from the 1133 HCC set. Node border colors (Green/red) denote periportal/perivenous attributes of mouse genes genes, respectively.(20) Node core colors are proportional to the PV/PP fold-change in the HCC set (Color key: lower right). Link thickness is proportional to correlation coefficients ( $>0.30$ in all networks; blue, positive; red, negative correlation). (B) The 550 genes representative of the four HCC subclasses discriminate between Hnf4a-KO and Hnf4a-WT mice.(31). Three gene clusters (black nodes on the left) denote: 1, upregulation in Hnf4aKO's and enrichment in human HCC STEM/ECM signatures; 2, upregulation in Hnf4a-WT's and enrichment in HCC PP genes; 3, genes poorly affected by Hnf4a status. Red, high expression, green, low expression.

$179 \times 191 \mathrm{~mm}(300 \times 300 \mathrm{DPI})$ 


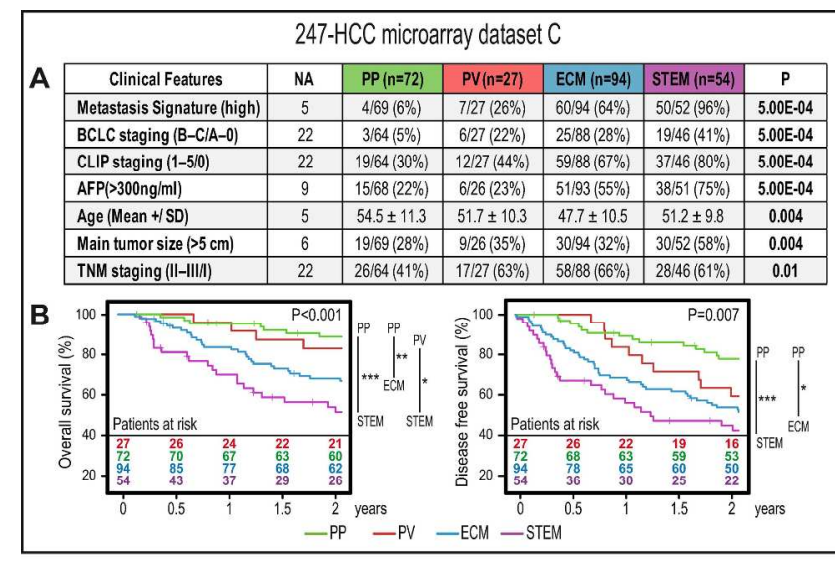

External Validation 210-HCC RNAseq dataset (TCGA-LIHC-US)
\begin{tabular}{|l|c|c|c|c|c|c|}
\hline C Clinical Features & NA & PP (N=58) & PV (N=42) & ECM (N=84) & STEM (N=26) & P \\
\hline CTNNB1 exon3 mutation (yes/no) & 80 & $3 / 39(8 \%)$ & $17 / 21(81 \%)$ & $5 / 52(10 \%)$ & $0 / 18(0 \%)$ & $1.51 E-11$ \\
\hline AFP (>Median) & 57 & $14 / 40(35 \%)$ & $6 / 34(18 \%)$ & $48 / 64(75 \%)$ & $9 / 15(60 \%)$ & $5.65 E-08$ \\
\hline Tumor grade (G3-G4/G1-G2) & 2 & $12 / 57(21 \%)$ & $14 / 42(33 \%)$ & $37 / 83(45 \%)$ & $19 / 26(73 \%)$ & $5.98 E-05$ \\
\hline Gender (Male) & 0 & $43 / 58(74 \%)$ & $38 / 42(90 \%)$ & $47 / 84(56 \%)$ & $16 / 26(62 \%)$ & $3.84 E-04$ \\
\hline TNM staging (T2-T3-T4/T1) & 0 & $22 / 58(39 \%)$ & $22 / 42(52 \%)$ & $44 / 84(52 \%)$ & $21 / 26(81 \%)$ & $4.48 E-03$ \\
\hline TNM staging (II-III-IVII) & 15 & $19 / 51(37 \%)$ & $21 / 39(54 \%)$ & $41 / 80(51 \%)$ & $20 / 25(80 \%)$ & $5.45 E-03$ \\
\hline Vascularinvasion & 31 & & & & & 0.01 \\
\hline None & & $42 / 53(79 \%)$ & $24 / 37(65 \%)$ & $49 / 72(68 \%)$ & $9 / 17(53 \%)$ & \\
\hline Micro & & $11 / 53(21 \%)$ & $13 / 37(35 \%)$ & $18 / 72(25 \%)$ & $4 / 17(23 \%)$ & \\
\hline Macro & & $0 / 53(0 \%)$ & $0 / 37(0 \%)$ & $5 / 72(7 \%)$ & $4 / 17(23 \%)$ & \\
\hline Age (Mean +1-SD) & 0 & 62.4112 .9 & $61.0 \perp 10.6$ & $56.3 \pm 13.4$ & 55.5113 .3 & 0.02 \\
\hline TP53 mutation (yes/no) & 80 & $8 / 39(21 \%)$ & $5 / 21(24 \%)$ & $20152(38 \%)$ & $10 / 18(56 \%)$ & 0.04 \\
\hline
\end{tabular}
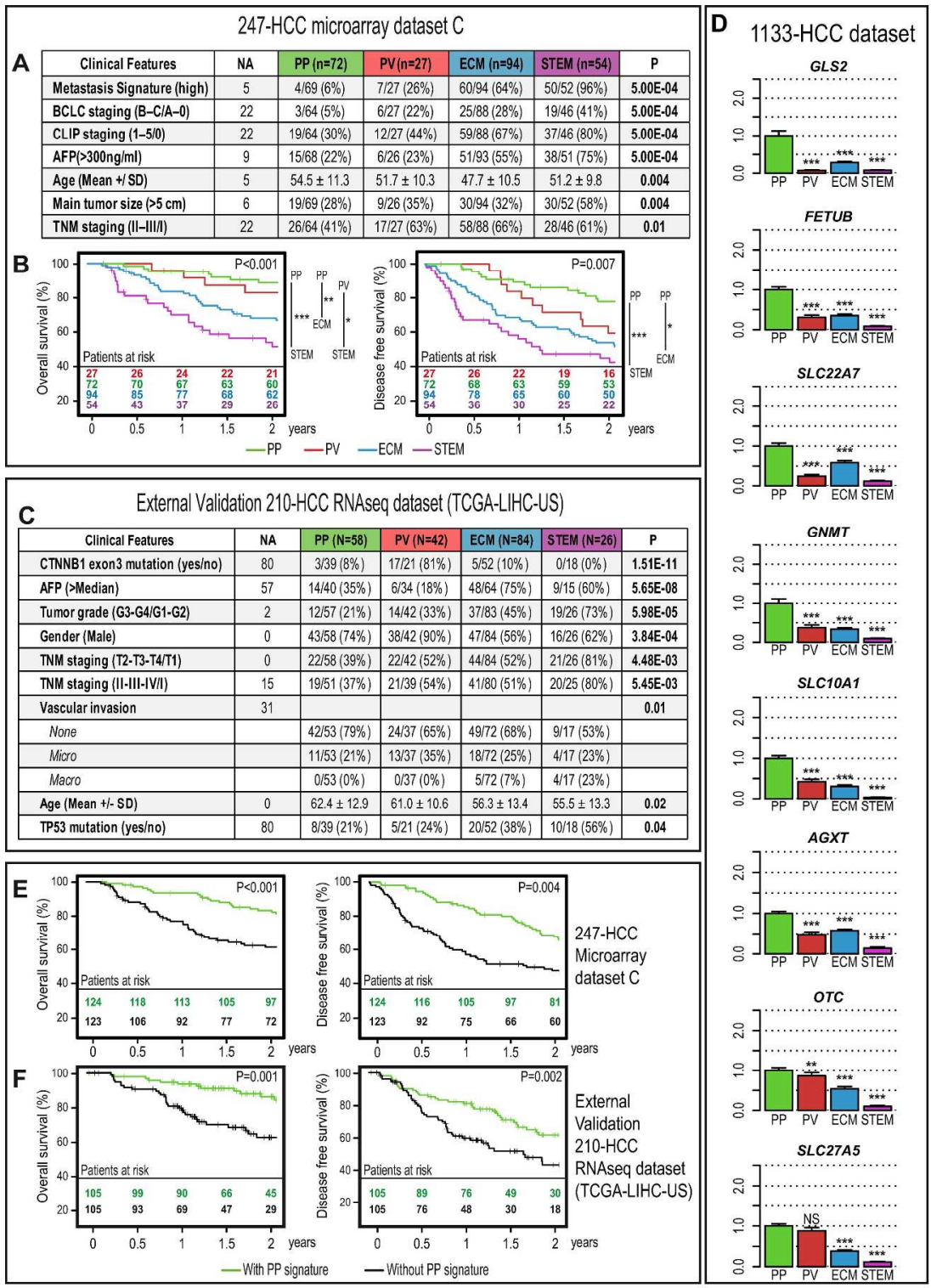

Désert et al., Figure 6

Fig. 6. Periportal-type HCCs show the most favorable clinical features and the highest early (2-year) disease-free and overall survival rates after resection. (A) Clinical features of HCC subclasses in a 247patient dataset(34). (B) Kaplan-Meier plots of subclass-specific overall and disease-free survival; $* \mathrm{P}<0.05$, $* * P<0.01, * * * P<0.001$. (C) Subclass-specific clinical features, CTNNB1 and TP53 mutation rates (full genome sequencing) in an external validation 210-HCC RNAseq dataset (TGCA-LIHC-US). (D) Subclassspecific mRNA expression levels of Periportal HCC signature genes in 1133 HCCs. (E, F) Kaplan-Meier plots of overall and disease-free survival in HCC patients with and without the Periportal HCC signature in two datasets. AFP, serum alpha-fetoprotein; NA, not available; BCLC (Barcelona Clinic Liver Cancer); CLIP (Cancer of the Liver Italian Program). Statistics: Fisher exact test (categorical variables); Student's t test (continuous variables); Log-rank test (survival analyses). 

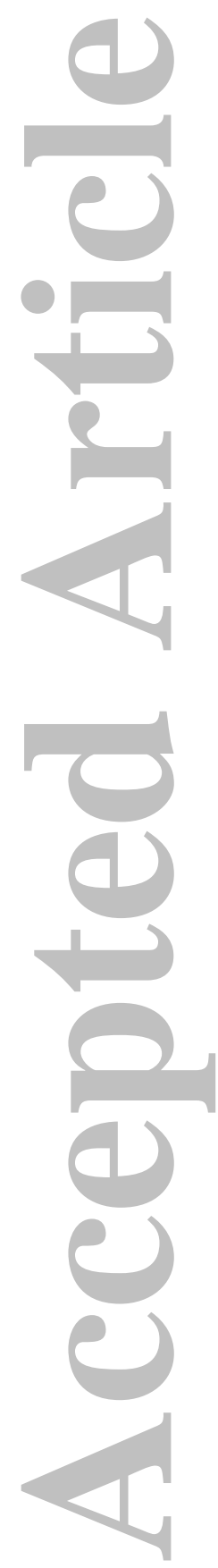

Hepatology

This article is protected by copyright. All rights reserved. 
A

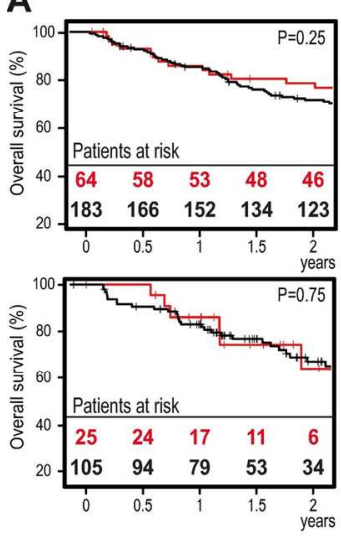

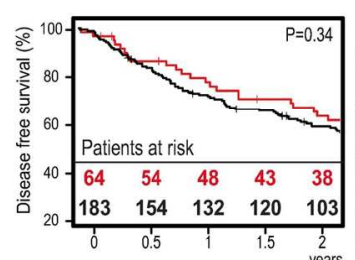

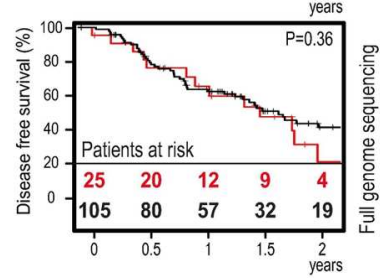

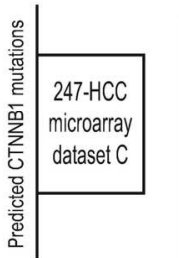

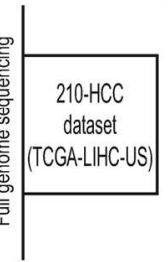

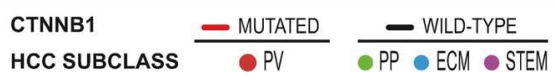

D

HCCs with wild-type CTNNB1

B

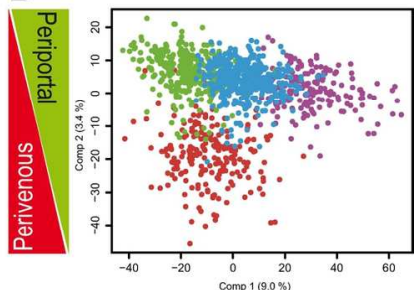

Hepatocyte-like

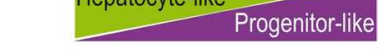

HCCs with mutated CTNNB1

Non-proliferation class

Perivenous subclass (19\%)

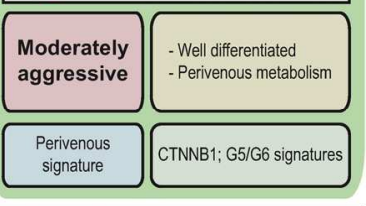

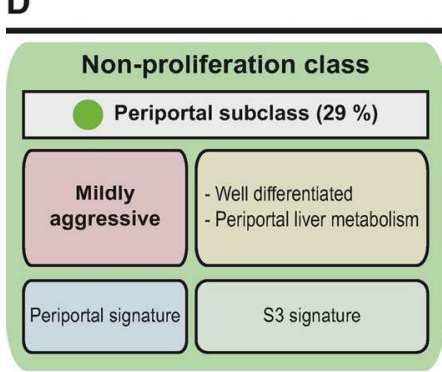

Proliferation class

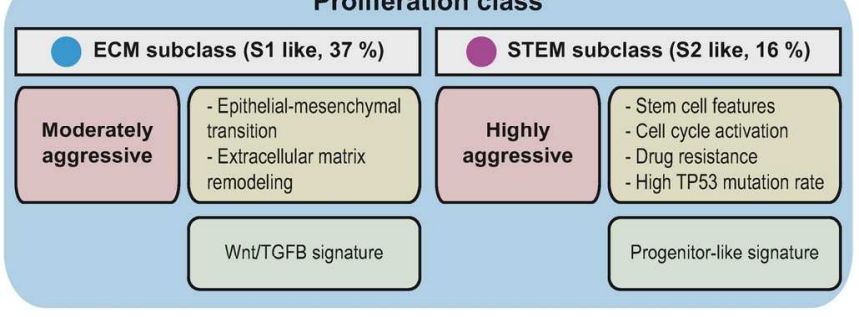

Désert et al., Figure 7

Fig. 7. Periportal-type HCCs and the survival paradox between CTNNB1-mutated and wild-type HCCs. (A) Kaplan-Meier plots and log-rank tests in two independent HCC datasets confirm similar outcome of HCCS carrying mutated (red) or wild-type (black) CTNNB1. Mutational status results from prediction in dataset C and full-genome sequencing in TCGA-LICH-US. (B) Multivariate discriminant analysis of the four HCC subclasses. In the first component, there is a grading from Periportal-type (green), through Perivenous(red) and ECM-types (blue), to STEM-type (purple). In the second component, Perivenous-type differs from other HCC subclasses. (C) HCCs with predominantly mutated CTNNB1 are moderately aggressive. (D) HCCs with predominantly wild-type CTNNB1 include mildly, moderately and highly aggressive subclasses. The \% of HCCs in each subclass is indicated in parentheses.

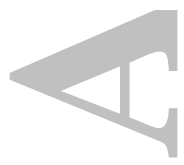

$155 \times 139 \mathrm{~mm}(300 \times 300 \mathrm{DPI})$ 
Table 1. Univariate and multivariate Cox's 2-year disease-free survival analyses in two independent HCC datasets.

\begin{tabular}{|c|c|c|c|c|c|c|}
\hline & \multirow{2}{*}{$\begin{array}{l}\text { Missing } \\
\text { values }\end{array}$} & \multirow{2}{*}{$\mathrm{N}(\%)$} & \multicolumn{2}{|c|}{ Univariate analysis } & \multicolumn{2}{|c|}{ Multivariate analysis } \\
\hline & & & $\mathrm{HR}(95 \% \mathrm{Cl})$ & Wald $P$ value & $\mathrm{HR}(95 \% \mathrm{Cl})$ & Wald $P$ value \\
\hline \multicolumn{7}{|c|}{ 247-HCC microarray dataset C } \\
\hline Sex (male) & 5 & $211(87 \%)$ & $2.38(1.25-4.53)$ & $8.41 \mathrm{E}-03$ & $2.25(1.16-4.33)$ & 0.01 \\
\hline Age $(>60 \mathrm{y})$ & 5 & $46(19 \%)$ & $1.02(0.66-1.56)$ & 0.94 & & \\
\hline HBV viral status (AVR) & 23 & $58(26 \%)$ & $1.34(0.91-1.96)$ & 0.14 & & \\
\hline $\operatorname{ALT}(>50 \mathrm{U} / \mathrm{L})$. & 5 & $100(41 \%)$ & $1.4(1-1.96)$ & 0.05 & & \\
\hline Main tumor size $(>5 \mathrm{~cm})$ & 6 & $88(37 \%)$ & $1.38(0.97-1.94)$ & 0.07 & $1.14(0.79-1.65)$ & 0.47 \\
\hline Multinodular & 5 & $52(21 \%)$ & $1.33(0.9-1.98)$ & 0.15 & $1.10(0.73-1.66)$ & 0.63 \\
\hline Cirrhosis & 5 & $223(92 \%)$ & $1.99(0.93-4.27)$ & 0.08 & & \\
\hline AFP $(>300 \mathrm{ng} / \mathrm{ml})$ & 9 & $110(46 \%)$ & $1.31(0.93-1.83)$ & 0.12 & $1.22(0.85-1.74)$ & 0.26 \\
\hline Periportal 8-gene signature & 0 & $124(50 \%)$ & $0.62(0.45-0.88)$ & $6.37 \mathrm{E}-03$ & $0.69(0.48-0.97)$ & 0.03 \\
\hline
\end{tabular}

\begin{tabular}{|c|c|c|c|c|c|c|}
\hline \multicolumn{7}{|c|}{$\begin{array}{c}\text { External validation 210-HCC RNAseq dataset } \\
\text { (TCGA-LIHC-US) }\end{array}$} \\
\hline Sex (male) & 0 & $144(69 \%)$ & $0.84(0.55-1.27)$ & 0.40 & & \\
\hline Age $(>60 y)$ & 0 & $104(50 \%)$ & $0.8(0.53-1.19)$ & 0.26 & & \\
\hline Patients from Asia & 6 & $71(35 \%)$ & $0.96(0.63-1.47)$ & 0.87 & & \\
\hline Patients from USA & 6 & $101(50 \%)$ & $1.09(0.73-1.63)$ & 0.67 & & \\
\hline Obesity & 18 & $32(17 \%)$ & $0.85(0.48-1.51)$ & 0.59 & & \\
\hline Tumor sample weight (>median) & 0 & $69(33 \%)$ & $1.73(1.11-2.7)$ & 0.02 & $0.98(0.54-1.8)$ & 0.96 \\
\hline Vascular invasion & 31 & $55(31 \%)$ & $1.68(1.05-2.69)$ & 0.03 & $1.52(0.92-2.5)$ & 0.10 \\
\hline Child pugh (>A) & 68 & $15(11 \%)$ & $1.44(0.65-3.18)$ & 0.36 & & \\
\hline AFP (>median) & 57 & $76(50 \%)$ & $1.16(0.72-1.85)$ & 0.55 & & \\
\hline Platelet count (>median) & 34 & $87(49 \%)$ & $1.49(0.95-2.34)$ & 0.08 & & \\
\hline Hepatic inflammation of adjacent tissue & 68 & $76(54 \%)$ & $1.03(0.64-1.65)$ & 0.92 & & \\
\hline Performance Status $(>0)$ & 40 & $77(45 \%)$ & $1.53(0.99-2.35)$ & 0.05 & & \\
\hline CTNNB1 exon3 mutation & 80 & $25(19 \%)$ & $1.33(0.74-2.36)$ & 0.34 & & \\
\hline TP53 mutation & 80 & $43(33 \%)$ & $1.4(0.84-2.32)$ & 0.20 & & \\
\hline Periportal 8-gene signature & 0 & $105(50 \%)$ & $0.53(0.36-0.8)$ & 0.003 & $0.59(0.37-0.94)$ & 0.02 \\
\hline
\end{tabular}


Supporting Table S2. See Supplementary Data.docx file for caption.

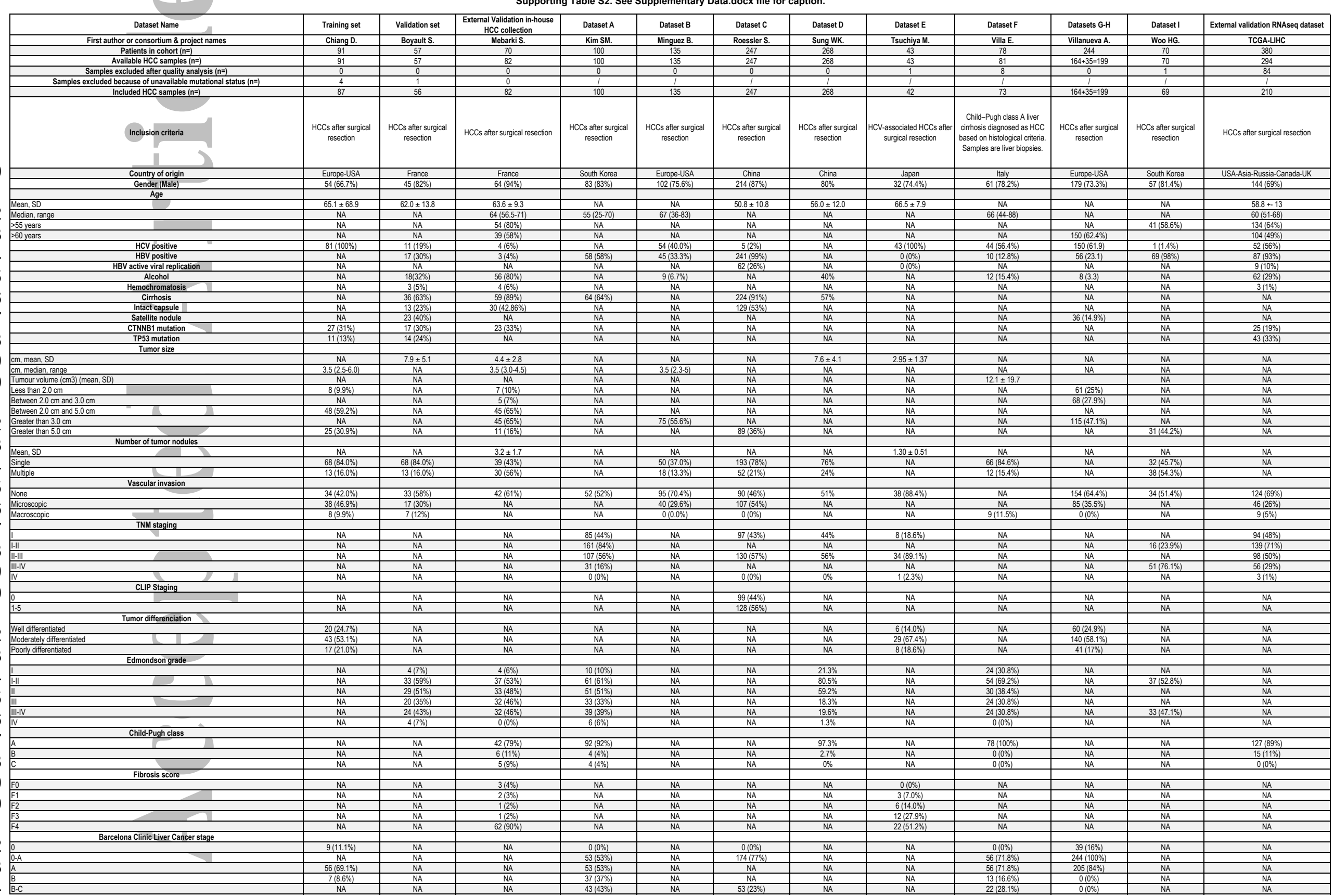

Hepatology

This article is protected by copyright. All rights reserved. 


\section{Page 51 of 143}

Hepatology

0
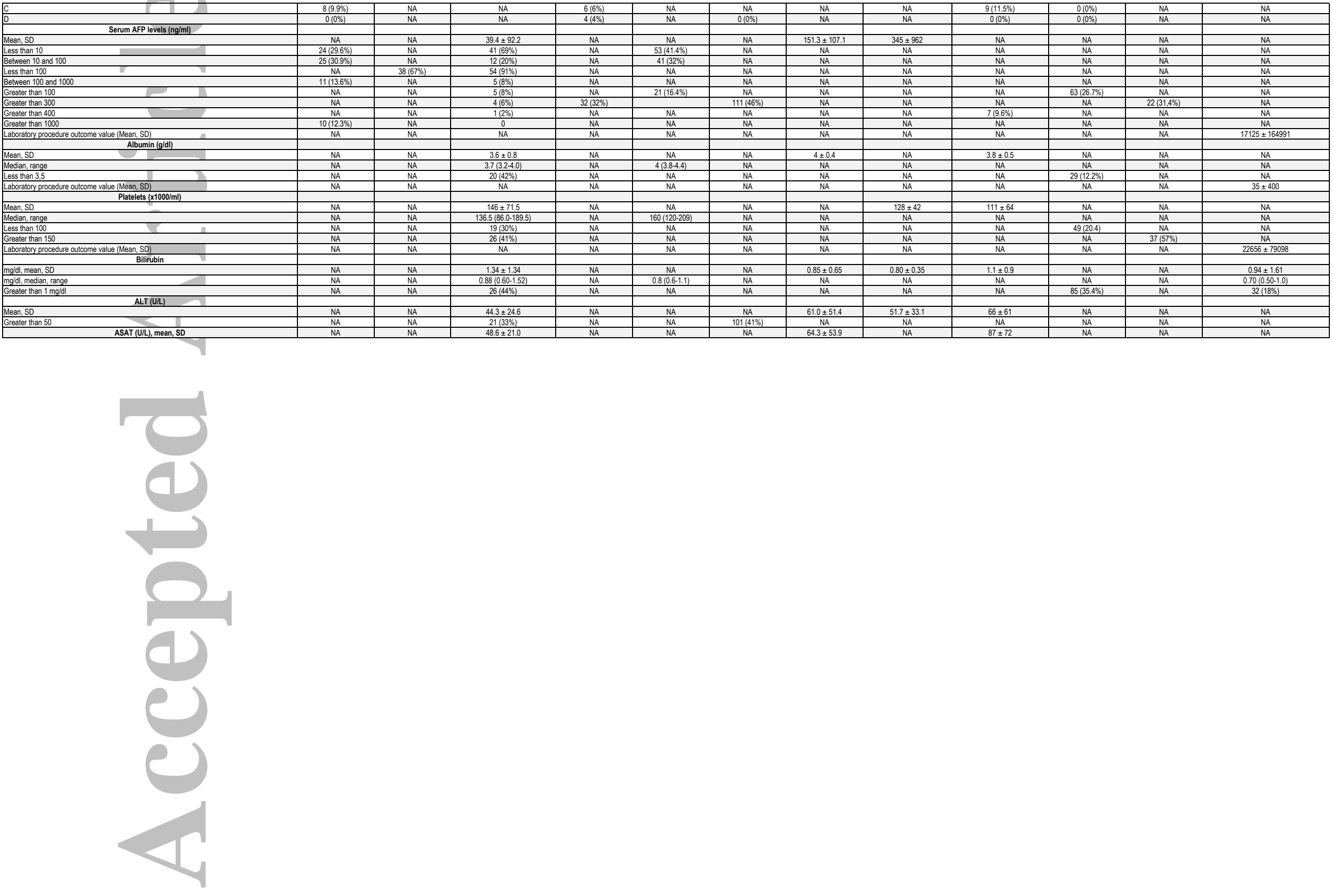

Hepatology

This article is protected by copyright. All rights reserved. 
Supporting Table S3. See Supplementary Data.docx file for caption.

\begin{tabular}{|c|c|c|c|c|c|c|c|c|c|c|c|c|c|}
\hline \multirow[b]{2}{*}{ Data source } & \multirow[b]{2}{*}{$\begin{array}{c}\text { Number of probes in the } \\
\text { array }\end{array}$} & \multirow[b]{2}{*}{$\begin{array}{l}\text { Number of genes } \\
\text { expressed over } \\
\text { background }\end{array}$} & \multicolumn{11}{|c|}{ Pearson's Correlations } \\
\hline & & & EPCAM-KRT19 & GS-HAL & GS-LGR5 & GS-ODAM & GS-VNN1 & LGR5-HAL & LGR5-ODAM & LGR5-VNN1 & HAL-ODAM & HAL-VNN1 & ODAM-VNN1 \\
\hline A & 48701 & 18758 & 0.67 & -0.52 & 0.58 & 0.67 & -0.43 & -0.49 & 0.56 & -0.54 & -0.57 & 0.38 & -0.51 \\
\hline$B$ & 24526 & 18277 & 0.27 & -0.51 & 0.42 & 0.71 & -0.42 & -0.48 & 0.33 & -0.36 & -0.50 & 0.28 & -0.43 \\
\hline$C$ & 22277 & 12509 & 0.51 & -0.46 & 0.58 & 0.55 & -0.20 & -0.29 & 0.43 & -0.34 & -0.51 & 0.33 & -0.30 \\
\hline $\mathrm{D}$ & 37582 & 18918 & 0.46 & -0.66 & 0.54 & 0.68 & -0.38 & -0.55 & 0.58 & -0.42 & -0.68 & 0.56 & -0.53 \\
\hline$E$ & 43376 & 18883 & 0.57 & -0.81 & 0.74 & 0.78 & -0.68 & -0.63 & 0.62 & -0.63 & -0.63 & 0.59 & -0.59 \\
\hline $\mathrm{F}$ & 43376 & 18962 & 0.84 & -0.50 & 0.41 & 0.42 & -0.51 & -0.64 & 0.62 & -0.53 & -0.45 & 0.50 & -0.46 \\
\hline G & 24526 & 18344 & 0.42 & $\begin{array}{l}-0.58 \\
\end{array}$ & 0.61 & 0.79 & -0.49 & -0.51 & 0.56 & -0.47 & -0.60 & 0.33 & $\begin{array}{l}-0.54 \\
\end{array}$ \\
\hline $\mathrm{H}$ & 48781 & 24919 & 0.78 & -0.56 & 0.82 & 0.68 & -0.72 & -0.54 & 0.51 & -0.57 & -0.55 & 0.63 & -0.73 \\
\hline 1 & 22277 & 12138 & 0.49 & -0.62 & 0.74 & 0.51 & -0.49 & -0.64 & 0.58 & -0.47 & -0.72 & 0.47 & -0.48 \\
\hline
\end{tabular}

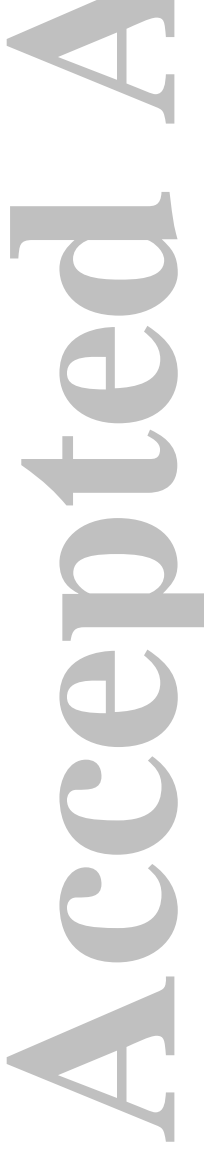

Hepatology

This article is protected by copyright. All rights reserved. 
Supporting Table S4. See Supplementary Data.docx file for caption.

\begin{tabular}{|c|c|c|c|c|c|}
\hline \multicolumn{2}{|r|}{ Training set } & \multicolumn{2}{|r|}{ Validation set } & \multicolumn{2}{|c|}{ External Validation: in-house HCC collection } \\
\hline ID & CTNNB1 exon3 mutationnal status & ID & CTNNB1 exon3 mutationnal status & ID & CTNNB1 exon3 mutationnal status \\
\hline GSM248688 & WT & HCC253 & beta-catenin_not_mutated & 1 & WT \\
\hline GSM248689 & WT & HCC399 & beta-catenin_mutated & 2 & WT \\
\hline GSM248690 & WT & HCC373 & beta-catenin_not_mutated & 3 & c.A121C/p.T41P \\
\hline GSM248691 & $233 A>A G, p . D 32 D / G$ & HCC189 & beta-catenin_not_mutated & 4 & c.C134T/p.S45F \\
\hline GSM248692 & 239G>GA,p.G34G/E & HCC210 & beta-catenin_not_mutated & 5 & c.A121C/p.T41P \\
\hline GSM248693 & 239G>GT,p.G34G/V & HCC313 & beta-catenin_not_mutated & 6 & WT \\
\hline GSM248694 & WT & HCC198 & beta-catenin_not_mutated & 7 & c.del107-109 p.H36-S37>P \\
\hline GSM248695 & $259 \mathrm{~A}>\mathrm{AG}, \mathrm{p} . \mathrm{T} 41 \mathrm{~T} / \mathrm{A}$ & HCC320 & beta-catenin_not_mutated & 8 & c.A121C/p.T41P \\
\hline GSM248696 & WT & HCC437 & beta-catenin_not_mutated & 9 & WT \\
\hline GSM248697 & WT & HCC060 & beta-catenin_not_mutated & 10 & WT \\
\hline GSM248698 & WT & HCC218 & beta-catenin_mutated & 11 & c.A95T/p.D32V \\
\hline GSM248699 & $233 A>A G, p . D 32 D / G$ & HCC121 & beta-catenin_mutated & 12 & WT \\
\hline GSM248700 & WT & HCC204 & beta-catenin_not_mutated & 13 & c.C134T/p.S45F \\
\hline GSM248701 & WT & HCC231 & beta-catenin_mutated & 14 & c.A107C/p.H36P \\
\hline GSM248702 & $236 \mathrm{C}>\mathrm{CT} \mid \mathrm{S} 33 \mathrm{~S} / \mathrm{F}$ & \begin{tabular}{l|l} 
PoolL \\
\end{tabular} & beta-catenin_not_mutated & 15 & c.C134T/p.S45F \\
\hline GSM248703 & WT & HCC441 & beta-catenin_mutated & 16 & WT \\
\hline GSM248704 & $271 \mathrm{~T}>\mathrm{TG}, \mathrm{p} . \mathrm{S} 45 \mathrm{~S} / \mathrm{A}$ & PoolJ & beta-catenin_not_mutated & 17 & WT \\
\hline GSM248705 & WT & HCC237 & beta-catenin_mutated & 18 & WT \\
\hline GSM248706 & $232 \mathrm{G}>\mathrm{GA} \mid \mathrm{D} 32 \mathrm{D} / \mathrm{N}$ & HCC206 & beta-catenin_not_mutated & 19 & WT \\
\hline GSM248707 & $247 \mathrm{~T}>\mathrm{TG} \mid \mathrm{S} 37 \mathrm{~S} / \mathrm{A}$ & HCC229 & beta-catenin_mutated & 20 & WT \\
\hline GSM248708 & $272 \mathrm{C}>\mathrm{T} \mid \mathrm{S} 45 \mathrm{~F}$ & $\mathrm{HCC} 013$ & beta-catenin_mutated & 21 & WT \\
\hline GSM248709 & WT & HCC137 & beta-catenin_mutated & 22 & WT \\
\hline GSM248710 & $248 \mathrm{C}>\mathrm{CG} \mid \mathrm{S} 37 \mathrm{~S} / \mathrm{C}$ & HCC205 & beta-catenin_not_mutated & 23 & c.G94T/p.D32Y \\
\hline GSM248711 & WT & HCC335 & beta-catenin_not_mutated & 24 & c.A107C/p.H36P \\
\hline GSM248712 & WT & PoolH & beta-catenin_mutated & 25 & WT \\
\hline GSM248713 & WT & HCC438 & beta-catenin_mutated & 26 & WT \\
\hline GSM248714 & WT & HCC322 & beta-catenin_not_mutated & 27 & WT \\
\hline GSM248715 & WT & HCC107 & beta-catenin_mutated & 28 & WT \\
\hline GSM248716 & WT & HCC242 & beta-catenin_not_mutated & 29 & WT \\
\hline GSM248717 & $236 \mathrm{C}>\mathrm{CG} \mid \mathrm{S} 33 \mathrm{~S} / \mathrm{C}$ & HCC333 & beta-catenin_not_mutated & 30 & WT \\
\hline GSM248718 & WT & HCC304 & beta-catenin_not_mutated & 31 & c.G94T/p.D32Y \\
\hline GSM248719 & WT & HCC433 & beta-catenin_not_mutated & 32 & WT \\
\hline GSM248720 & $247 \mathrm{~T}>\mathrm{TG} \mid \mathrm{S} 37 \mathrm{~S} / \mathrm{A}$ & HCC081 & beta-catenin_mutated & 33 & WT \\
\hline GSM248721 & WT & HCC168 & beta-catenin_not_mutated & 34 & WT \\
\hline GSM248722 & WT & HCC230 & beta-catenin_not_mutated & 35 & WT \\
\hline GSM248723 & WT & HCC405 & beta-catenin_not_mutated & 36 & WT \\
\hline GSM248724 & WT & HCC197 & beta-catenin_not_mutated & 37 & c.A107C/p.H36P \\
\hline GSM248725 & WT & HCC314 & beta-catenin_not_mutated & 38 & WT \\
\hline GSM248726 & $248 \mathrm{C}>\mathrm{CT} \mid \mathrm{S} 37 \mathrm{~S} / \mathrm{F}$ & HCC208 & beta-catenin_not_mutated & 39 & c.C122T/p.T41I \\
\hline GSM248727 & WT & HCC301 & beta-catenin_mutated & 40 & WT \\
\hline GSM248728 & WT & HCC016 & beta-catenin_not_mutated & 41 & WT \\
\hline GSM248729 & WT & HCC164 & beta-catenin_not_mutated & 42 & WT \\
\hline GSM248731 & $271 \mathrm{~T}>\mathrm{TG}, \mathrm{p} . \mathrm{S} 45 \mathrm{~S} / \mathrm{A}$ & HCC010 & beta-catenin_not_mutated & 43 & c.A121C/p.T41P \\
\hline GSM248732 & WT & HCC252 & beta-catenin_mutated & 44 & WT \\
\hline GSM248733 & $233 \mathrm{~A}>\mathrm{AT} \mid \mathrm{D} 32 \mathrm{D} / \mathrm{N}$ & HCC170 & beta-catenin_not_mutated & 45 & WT \\
\hline GSM248734 & $233 \mathrm{~A}>\mathrm{AG}, \mathrm{p} . \mathrm{D} 32 \mathrm{D} / \mathrm{G}$ & HCC358 & beta-catenin_mutated & 46 & WT \\
\hline GSM248735 & 0 & HCC195 & beta-catenin_not_mutated & 47 & c.C122T/p.T41I \\
\hline GSM248736 & $236 \mathrm{C}>\mathrm{CT}, \mathrm{p} . \mathrm{S} 33 \mathrm{~S} / \mathrm{F}$ & HCC402 & beta-catenin_not_mutated & 48 & WT \\
\hline GSM248737 & WT & HCC305 & beta-catenin_not_mutated & 49 & WT \\
\hline GSM248738 & WT & Pooll & beta-catenin_not_mutated & 50 & WT \\
\hline GSM248739 & WT & HCC018 & beta-catenin_not_mutated & 51 & WT \\
\hline GSM248740 & WT & HCC429 & beta-catenin_not_mutated & 52 & WT \\
\hline GSM248741 & WT & HCC432 & beta-catenin_not_mutated & 53 & c.G101T/p.G34V \\
\hline GSM248742 & $260 \mathrm{C}>\mathrm{CT} \mid \mathrm{T} 41 \mathrm{~T} / \mathrm{I}$ & PoolK & beta-catenin_not_mutated & 54 & WT \\
\hline GSM248743 & $232 G>G A, p . D 32 D / N$ & HCC309 & beta-catenin_not_mutated & 55 & WT \\
\hline GSM248744 & $235 \mathrm{~T}>\mathrm{TC} \mid \mathrm{S} 33 \mathrm{~S} / \mathrm{P}$ & HCC191 & beta-catenin_mutated & 56 & WT \\
\hline GSM248745 & WT & & & 57 & WT \\
\hline GSM248746 & WT & & & 58 & WT \\
\hline GSM248747 & $232 \mathrm{G}>\mathrm{GT} \mid \mathrm{D} 32 \mathrm{D} / \mathrm{Y}$ & & & 59 & WT \\
\hline
\end{tabular}




\begin{tabular}{|c|c|}
\hline GSM248748 & WT \\
\hline GSM248749 & 248C>CT | S37S/F \\
\hline GSM248750 & WT \\
\hline GSM248751 & WT \\
\hline GSM248753 & WT \\
\hline GSM248754 & WT \\
\hline GSM248755 & WT \\
\hline GSM248756 & WT \\
\hline GSM248757 & WT \\
\hline GSM248758 & WT \\
\hline GSM248759 & 234_245het_delCTCTGGAATCCA \\
\hline GSM248760 & WT \\
\hline GSM248761 & WT \\
\hline GSM248762 & WT \\
\hline GSM248763 & WT \\
\hline GSM248764 & 232G>GA | D32D/N \\
\hline GSM248765 & WT \\
\hline GSM248766 & WT \\
\hline GSM248767 & 248C>CG S37S/C \\
\hline GSM248769 & WT \\
\hline GSM248771 & WT \\
\hline GSM248772 & WT \\
\hline GSM248773 & WT \\
\hline GSM248774 & WT \\
\hline GSM248775 & WT \\
\hline GSM248776 & WT \\
\hline GSM248777 & WT \\
\hline GSM248778 & \\
\hline
\end{tabular}

\begin{tabular}{|l|c|}
\hline 60 & WT \\
\hline 61 & WT \\
\hline 62 & WT \\
\hline 63 & WT \\
\hline 64 & c.C134A/p.S45Y \\
\hline 65 & WT \\
\hline 66 & WT \\
\hline 67 & c.A95T/p.D32V \\
\hline 68 & c.A95G/p.D32G \\
\hline 69 & WT \\
\hline 70 & c.G101T/p.G34V \\
\hline 71 & c.A121G/p.T41A \\
\hline 72 & WT \\
\hline 73 & WT \\
\hline 74 & WT \\
\hline 75 & c.A95G/p.D32G \\
\hline 76 & WT \\
\hline 77 & WT \\
\hline 78 & c.C110G/p.S37C \\
\hline 79 & WT \\
\hline 80 & WT \\
\hline 81 & WT \\
\hline 82 & c.G94T/p.D32Y \\
\hline & \\
\hline
\end{tabular}

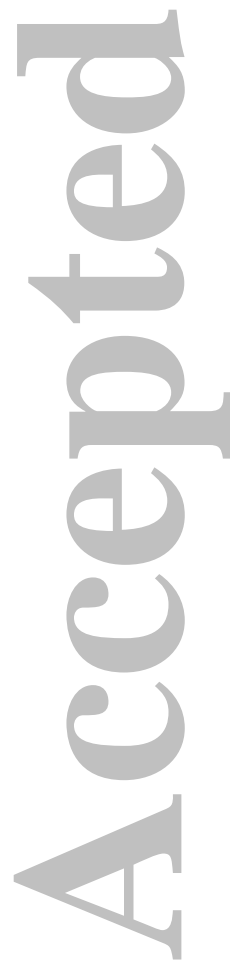


Supporting Table S5. See Supplementary Data.docx file for caption.

\begin{tabular}{|c|c|c|c|c|c|c|}
\hline PREDICTION OF CTNNB1 MUTATIONS & \multicolumn{5}{|c|}{ Predicted CTNNB1 mutations } & \multirow{2}{*}{$\begin{array}{c}\text { Sanger-sequenced CTNNB1 } \\
\% \text { of CTNNB1 mutations }\end{array}$} \\
\hline Data source & $\begin{array}{l}\text { AUROC } \\
\text { (Score) }\end{array}$ & $\begin{array}{l}\text { Best } \\
\text { common } \\
\text { threshold }\end{array}$ & Sensitivity & Specificity & $\begin{array}{c}\% \text { of predicted CTNNB1 } \\
\text { mutations }\end{array}$ & \\
\hline Training set & 0.90 & 8 & 0.82 & 0.83 & $38 \%$ & $31 \%$ \\
\hline Validation set & 0.92 & 8 & 0.88 & 0.89 & $28 \%$ & $30 \%$ \\
\hline $\mathrm{A}$ & & & & & $24 \%$ & $\mathrm{NA}$ \\
\hline $\mathbf{B}$ & & & & & $30 \%$ & NA \\
\hline $\mathrm{C}$ & & & & & $25 \%$ & NA \\
\hline D & & & & & $25 \%$ & NA \\
\hline$E$ & & & & & $40 \%$ & NA \\
\hline $\mathbf{F}$ & & & & & $20 \%$ & NA \\
\hline $\mathbf{G}$ & & & & & $31 \%$ & NA \\
\hline $\mathrm{H}$ & & & & & $34 \%$ & NA \\
\hline $\mathrm{I}$ & & & & & $28 \%$ & NA \\
\hline
\end{tabular}


Supporting Table S6. See Supplementary Data.docx file for caption.

\begin{tabular}{|c|c|c|c|c|c|}
\hline \multirow{2}{*}{ Dataset } & \multicolumn{4}{|c|}{ HCC Subclasses } & Number of \\
\cline { 2 - 4 } & GREEN & RED & BLUE & PURPLE & HCC \\
\hline A & $3 \%$ & $79 \%$ & $0 \%$ & $19 \%$ & 100 \\
\hline B & $10 \%$ & $78 \%$ & $3 \%$ & $0 \%$ & 135 \\
\hline C & $10 \%$ & $89 \%$ & $6 \%$ & $50 \%$ & 247 \\
\hline D & $4 \%$ & $98 \%$ & $12 \%$ & $28 \%$ & 268 \\
\hline E & $9 \%$ & $100 \%$ & $17 \%$ & $20 \%$ & 42 \\
\hline F & $7 \%$ & $89 \%$ & $12 \%$ & $18 \%$ & 73 \\
\hline G & $3 \%$ & $65 \%$ & $6 \%$ & $0 \%$ & 164 \\
\hline H & $17 \%$ & $67 \%$ & $0 \%$ & $0 \%$ & 35 \\
\hline I & $5 \%$ & $93 \%$ & $0 \%$ & $29 \%$ & 69 \\
\hline Total & $6 \%$ & $84 \%$ & $7 \%$ & $28 \%$ & 1133 \\
\hline
\end{tabular}




\section{Supporting Table S7. See Supplementary Data.docx file for caption.}

\begin{tabular}{|c|c|c|c|}
\hline \multicolumn{4}{|l|}{ PERIPORTAL CLUSTER } \\
\hline \multicolumn{4}{|l|}{ Unsupervised GSEA } \\
\hline NAME & SIZE & NES & NOM p-val \\
\hline HOSHIDA_LIVER_CANCER_SURVIVAL_DN & 90 & 2.19 & 0 \\
\hline ACEVEDO_LIVER_CANCER_DN & 334 & 2.10 & 0 \\
\hline HOSHIDA_LIVER_CANCER_SUBCLASS_S3 & 217 & 2.10 & 0 \\
\hline CAIRO_LIVER_DEVELOPMENT_DN & 178 & 2.03 & 0 \\
\hline BURTON_ADIPOGENESIS_6 & 136 & 2.02 & 0 \\
\hline REACTOME_METABOLISM_OF_AMINO_ACIDS_AND_DERIVATIVES & 137 & 2.00 & 0 \\
\hline HALLMARK_XENOBIOTIC_METABOLISM & 157 & 1.99 & 0 \\
\hline REACTOME_LIPID_DIGESTION_MOBILIZATION_AND_TRANSPORT & 34 & 1.99 & 0 \\
\hline BOCHKIS_FOXA2_TARGETS & 265 & 1.96 & 0 \\
\hline ACEVEDO_LIVER_TUMOR_VS_NORMAL_ADJACENT_TISSUE_DN & 180 & 1.95 & 0 \\
\hline REACTOME_GLUCOSE_METABOLISM & 45 & 1.95 & 0 \\
\hline HALLMARK_ADIPOGENESIS & 145 & 1.94 & 0.0041 \\
\hline CAIRO_HEPATOBLASTOMA_DN & 203 & 1.94 & 0 \\
\hline HALLMARK_OXIDATIVE_PHOSPHORYLATION & 142 & 1.92 & 0.030 \\
\hline HALLMARK_FATTY_ACID_METABOLISM & 108 & 1.92 & 0 \\
\hline CHIANG_LIVER_CANCER_SUBCLASS_POLYSOMY7_UP & 43 & 1.91 & 0 \\
\hline BOYAULT_LIVER_CANCER_SUBCLASS_G1_DN & 35 & 1.91 & 0 \\
\hline REACTOME_GLUCONEOGENESIS & 21 & 1.91 & 0 \\
\hline HSIAO_LIVER_SPECIFIC_GENES & 193 & 1.90 & 0 \\
\hline LUCAS_HNF4A_TARGETS_UP & 44 & 1.89 & 0.0060 \\
\hline BOYAULT_LIVER_CANCER_SUBCLASS_G3_DN & 39 & 1.87 & 0 \\
\hline LEE_LIVER_CANCER_MYC_TGFA_DN & 54 & 1.87 & 0 \\
\hline KEGG_CYSTEINE_AND_METHIONINE_METABOLISM & 24 & 1.86 & 0.0021 \\
\hline HALLMARK_BILE_ACID_METABOLISM & 85 & 1.86 & 0 \\
\hline CHIANG_LIVER_CANCER_SUBCLASS_UNANNOTATED_UP & 51 & 1.86 & 0 \\
\hline HALLMARK_PEROXISOME & 80 & 1.81 & 0.0043 \\
\hline LEE_LIVER_CANCER_SURVIVAL_UP & 112 & 1.79 & 0 \\
\hline BOYAULT_LIVER_CANCER_SUBCLASS_G123_DN & 40 & 1.78 & 0 \\
\hline KEGG_COMPLEMENT_AND_COAGULATION_CASCADES & 59 & 1.78 & 0.0020 \\
\hline KEGG_PYRUVATE_METABOLISM & 30 & 1.77 & 0.0060 \\
\hline CHIANG_LIVER_CANCER_SUBCLASS_PROLIFERATION_DN & 121 & 1.75 & 0 \\
\hline LEE_LIVER_CANCER_MYC_E2F1_DN & 51 & 1.73 & 0 \\
\hline LEE_LIVER_CANCER_MYC_DN & 44 & 1.72 & 0.0041 \\
\hline LEE_LIVER_CANCER_E2F1_DN & 47 & 1.70 & 0 \\
\hline YAMASHITA_LIVER_CANCER_STEM_CELL_DN & 59 & 1.70 & 0.0020 \\
\hline REACTOME_PHASE_II_CONJUGATION & 36 & 1.70 & 0.0021 \\
\hline REACTOME_FATTY_ACID_TRIACYLGLYCEROL_AND_KETONE_BODY_METABOLISM & 108 & 1.69 & 0.0079 \\
\hline REACTOME_COMPLEMENT_CASCADE & 24 & 1.69 & 0.0059 \\
\hline KEGG_DRUG_METABOLISM_OTHER_ENZYMES & 24 & 1.69 & 0.0020 \\
\hline PID_HNF3B_PATHWAY & 31 & 1.69 & 0.012 \\
\hline HOSHIDA_LIVER_CANCER_LATE_RECURRENCE_DN & 51 & 1.69 & 0.0020 \\
\hline LEE_LIVER_CANCER_DENA_DN & 58 & 1.67 & 0.0060 \\
\hline CAIRO_HEPATOBLASTOMA_CLASSES_DN & 166 & 1.65 & 0.012 \\
\hline KEGG_HISTIDINE_METABOLISM & 20 & 1.64 & 0.011 \\
\hline MOOTHA_GLUCONEOGENESIS & 25 & 1.63 & 0.014 \\
\hline TANG_SENESCENCE_TP53_TARGETS_UP & 27 & 1.63 & 0.0040 \\
\hline WANG_CLASSIC_ADIPOGENIC_TARGETS_OF_PPARG & 17 & 1.63 & 0.017 \\
\hline REACTOME_HDL_MEDIATED_LIPID_TRANSPORT & 15 & 1.62 & 0.029 \\
\hline BOYAULT_LIVER_CANCER_SUBCLASS_G6_DN & 16 & 1.60 & 0.033 \\
\hline
\end{tabular}


\begin{tabular}{|l}
\hline HALLMARK_COAGULATION \\
\hline REACTOME_BILE_ACID_AND_BILE_SALT_METABOLISM
\end{tabular}

\begin{tabular}{|l|l|l|}
\hline 119 & 1.57 & 0.024 \\
\hline 22 & 1.51 & 0.031 \\
\hline
\end{tabular}

\section{Supervised GSEA}

GOUGELET_PERIPORTAL_METABOLISM

\begin{tabular}{l|l}
142 & 1.95 \\
\hline
\end{tabular}

0

\begin{tabular}{|c|c|c|c|}
\hline \multicolumn{4}{|c|}{ PERIVENOUS CLUSTER } \\
\hline \multicolumn{4}{|c|}{ Unsupervised GSEA } \\
\hline NAME & SIZE & NES & NOM p-val \\
\hline BOYAULT_LIVER_CANCER_SUBCLASS_G6_UP & 51 & 2.06 & 0 \\
\hline CHIANG_LIVER_CANCER_SUBCLASS_CTNNB1_UP & 110 & 1.97 & 0 \\
\hline SMITH LIVER CANCER & 31 & 1.86 & 0.002 \\
\hline BOYAULT_LIVER_CANCER_SUBCLASS_G1_DN & 35 & 1.86 & 0.008 \\
\hline CHIANG_LIVER_CANCER_SUBCLASS_INTERFERON_DN & 26 & 1.84 & 0 \\
\hline BIOCARTA_WNT_PATHWAY & 24 & 1.79 & 0.0095 \\
\hline WOO_LIVER_CANCER_RECURRENCE_DN & 61 & 1.78 & 0 \\
\hline LEE_LIVER_CANCER_DENA_DN & 58 & 1.74 & 0.0020 \\
\hline $\begin{array}{l}\text { LEE_LIVER_CANCER_MYC_UP } \\
\end{array}$ & 37 & 1.73 & 0 \\
\hline KANG_DOXORUBICIN_RESISTANCE_DN & 17 & 1.72 & 0.016 \\
\hline WANG_CLASSIC_ADIPOGENIC_TARGETS_OF_PPARG & 17 & 1.71 & 0.0020 \\
\hline LEE_LIVER_CANCER_SURVIVAL_UP & 112 & 1.70 & 0.0040 \\
\hline KEGG_ADHERENS_JUNCTION & 60 & 1.68 & 0.0061 \\
\hline KEGG_FATTY_ACID_METABOLISM & 29 & 1.67 & 0.0081 \\
\hline REACTOME_METABOLISM_OF_VITAMINS_AND_COFACTORS & 34 & 1.65 & 0.014 \\
\hline BIOCARTA_MTOR_PATHWAY & 18 & 1.63 & 0.047 \\
\hline CHIANG_LIVER_CANCER_SUBCLASS_PROLIFERATION_DN & 121 & 1.61 & 0.010 \\
\hline BOYAULT_LIVER_CANCER_SUBCLASS_G123_DN & 40 & 1.61 & 0.016 \\
\hline REACTOME_BILE_ACID_AND_BILE_SALT_METABOLISM & 22 & 1.61 & 0.014 \\
\hline PID_HDAC_CLASSII_PATHWAY & 24 & 1.61 & 0.041 \\
\hline PID_MYC_PATHWAY & 21 & 1.60 & 0.056 \\
\hline LIU_LIVER_CANCER & 21 & 1.58 & 0.040 \\
\hline CAIRO_HEPATOBLASTOMA_CLASSES_DN & 166 & 1.56 & 0.042 \\
\hline KEGG_PPAR_SIGNALING_PATHWAY & 52 & 1.51 & 0.048 \\
\hline PARENT_MTOR_SIGNALING_UP & 408 & 1.38 & 0.033 \\
\hline
\end{tabular}

GOUGELET_PERIVEINOUS_METABOLISM

\section{Supervised GSEA}

\begin{tabular}{|l|c|c|c|}
\hline \multicolumn{1}{|c|}{ EXTRACELLULAR MATRIX CLUSTER } & \multicolumn{3}{c|}{ Unsupervised GSEA } \\
\multicolumn{1}{|c|}{ NAME } & SIZE & NES & NOM p-val \\
\hline & 77 & 2.06 & 0.0020 \\
\hline BOYAULT_LIVER_CANCER_SUBCLASS_G1_UP & 96 & 2.01 & 0 \\
\hline ZHONG_SECRETOME_OF_LUNG_CANCER_AND_FIBROBLAST & 65 & 1.88 & 0.0019 \\
\hline REACTOME_INTEGRIN_CELL_SURFACE_INTERACTIONS & 25 & 1.87 & 0.0019 \\
\hline BIOCARTA_MYOSIN_PATHWAY & 300 & 1.84 & 0 \\
\hline LIAO_METASTASIS & 39 & 1.84 & 0 \\
\hline BORLAK_LIVER_CANCER_EGF_UP & 30 & 1.82 & 0.0039 \\
\hline LANDIS_BREAST_CANCER_PROGRESSION_UP & 59 & 1.82 & 0 \\
\hline GOTZMANN_EPITHELIAL_TO_MESENCHYMAL_TRANSITION_UP & 32 & 1.82 & 0.0062 \\
\hline BOYAULT_LIVER_CANCER_SUBCLASS_G12_UP & 118 & 1.81 & 0 \\
\hline CHIANG_LIVER_CANCER_SUBCLASS_CTNNB1_DN & 61 & 1.81 & 0 \\
\hline HOSHIDA_LIVER_CANCER_SURVIVAL_UP & 19 & 1.78 & 0 \\
\hline REACTOME_DEGRADATION_OF_THE_EXTRACELLULAR_MATRIX & 138 & 1.78 & 0.001980198 \\
\hline NABA_ECM_REGULATORS & &
\end{tabular}




\begin{tabular}{|c|c|c|c|}
\hline HUMMERICH_SKIN_CANCER_PROGRESSION_UP & 66 & 1.78 & 0 \\
\hline $\begin{array}{l}\text { HOSHIDA_LIVER_CANCER_LATE_RECURRENCE_UP } \\
\end{array}$ & 43 & 1.78 & 0.0019 \\
\hline ROZANOV_MMP14_TARGETS_SUBSET & 26 & 1.77 & 0.0040 \\
\hline REACTOME_HEPARAN_SULFATE_HEPARIN_HS_GAG_METABOLISM & 30 & 1.77 & 0 \\
\hline WOO_LIVER_CANCER_RECURRENCE_UP & 83 & 1.76 & 0 \\
\hline REACTOME_GLYCOSAMINOGLYCAN_METABOLISM & 67 & 1.75 & 0.0019 \\
\hline PID_RHOA_REG_PATHWAY & 33 & 1.75 & 0.0078 \\
\hline LI_WILMS_TUMOR_VS_FETAL_KIDNEY_2_DN & 43 & 1.75 & 0.0039 \\
\hline JECHLINGER_EPITHELIAL_TO_MESENCHYMAL_TRANSITION_UP & 60 & 1.75 & 0 \\
\hline LEE_TARGETS_OF_PTCH1_AND_SUFU_DN & 57 & 1.74 & 0 \\
\hline NABA_CORE_MATRISOME & 143 & 1.72 & 0.0038 \\
\hline RAMASWAMY_METASTASIS_UP & 43 & 1.72 & 0.011 \\
\hline PID_INTEGRIN3_PATHWAY & 34 & 1.71 & 0.0078 \\
\hline RUIZ_TNC_TARGETS_UP & 121 & 1.71 & 0.011 \\
\hline NABA_ECM_GLYCOPROTEINS & 101 & 1.71 & 0.0038 \\
\hline COULOUARN_TEMPORAL_TGFB1_SIGNATURE_UP & 71 & 1.70 & 0.0078 \\
\hline LEE_LIVER_CANCER_DENA_UP & 52 & 1.70 & 0.0020 \\
\hline REACTOME_EXTRACELLULAR_MATRIX_ORGANIZATION & 56 & 1.70 & 0.0078 \\
\hline PID_SYNDECAN_1_PATHWAY & 36 & 1.69 & 0.0039 \\
\hline TURASHVILI_BREAST_LOBULAR_CARCINOMA_VS_DUCTAL_NORMAL_UP & 45 & 1.69 & 0.0019 \\
\hline GERHOLD_ADIPOGENESIS_DN & 52 & 1.68 & 0.013 \\
\hline CHARAFE_BREAST_CANCER_BASAL_VS_MESENCHYMAL_UP & 70 & 1.68 & 0.0040 \\
\hline ROESSLER_LIVER_CANCER_METASTASIS_DN & 30 & 1.68 & 0.0020 \\
\hline BOYAULT_LIVER_CANCER_SUBCLASS_G2 & 21 & 1.68 & 0.012 \\
\hline LIEN_BREAST_CARCINOMA_METAPLASTIC & 22 & 1.67 & 0.0019 \\
\hline VERHAAK_GLIOBLASTOMA_MESENCHYMAL & 170 & 1.67 & 0.015 \\
\hline CROONQUIST_STROMAL_STIMULATION_UP & 42 & 1.67 & 0.004 \\
\hline HARRIS_BRAIN_CANCER_PROGENITORS & 26 & 1.67 & 0.0040 \\
\hline LEE_LIVER_CANCER_MYC_TGFA_UP & 45 & 1.67 & 0.0061 \\
\hline NGUYEN_NOTCH1_TARGETS_DN & 61 & 1.67 & 0.0020 \\
\hline REACTOME_NCAM1_INTERACTIONS & 25 & 1.66 & 0.0039 \\
\hline NABA_MATRISOME_ASSOCIATED & 394 & 1.66 & 0 \\
\hline KEGG_ECM_RECEPTOR_INTERACTION & 65 & 1.65 & 0.0059 \\
\hline HOSHIDA_LIVER_CANCER_SUBCLASS_S1 & 192 & 1.65 & 0.017 \\
\hline KEGG_FOCAL_ADHESION & 151 & 1.65 & 0.010 \\
\hline REACTOME_AXON_GUIDANCE & 177 & 1.65 & 0.0039 \\
\hline VERRECCHIA_EARLY_RESPONSE_TO_TGFB1 & 43 & 1.64 & 0.025 \\
\hline BOQUEST_STEM_CELL_UP & 197 & 1.64 & 0.008 \\
\hline PID_INTEGRIN1_PATHWAY & 52 & 1.64 & 0.023 \\
\hline ASTIER_INTEGRIN_SIGNALING & 37 & 1.63 & 0.018 \\
\hline BOYAULT_LIVER_CANCER_SUBCLASS_G6_DN & 16 & 1.63 & 0.028 \\
\hline LEE_LIVER_CANCER_E2F1_UP & 50 & 1.63 & 0.010 \\
\hline VERRECCHIA_DELAYED_RESPONSE_TO_TGFB1 & 29 & 1.62 & 0.0077 \\
\hline ALONSO_METASTASIS_EMT_UP & 27 & 1.62 & 0.038 \\
\hline PID_IL6_7_PATHWAY & 40 & 1.62 & 0.010 \\
\hline GENTLES_LEUKEMIC_STEM_CELL_UP & 15 & 1.62 & 0.0079 \\
\hline DASU_IL6_SIGNALING_UP & 47 & 1.61 & 0.010 \\
\hline PID_INTEGRIN2_PATHWAY & 21 & 1.61 & 0.029 \\
\hline PID_AVB3_INTEGRIN_PATHWAY & 55 & 1.61 & 0.021 \\
\hline TONKS_TARGETS_OF_RUNX1_RUNX1T1_FUSION_ERYTHROCYTE_UP & 126 & 1.61 & 0.010 \\
\hline ROZANOV_MMP14_TARGETS_UP & 151 & 1.61 & 0.0093 \\
\hline BOYAULT_LIVER_CANCER_SUBCLASS_G123_UP & 32 & 1.61 & 0.045 \\
\hline ANASTASSIOU_CANCER_MESENCHYMAL_TRANSITION_SIGNATURE & 53 & 1.61 & 0.0077 \\
\hline BYSTRYKH_HEMATOPOIESIS_STEM_CELL_SCP2_QTL_TRANS & 15 & 1.60 & 0.012 \\
\hline
\end{tabular}




\begin{tabular}{|c|c|c|c|}
\hline REACTOME_NCAM_SIGNALING_FOR_NEURITE_OUT_GROWTH & 45 & 1.60 & 0.014 \\
\hline LEE_AGING_MUSCLE_DN & 33 & 1.57 & 0.032 \\
\hline CHIANG_LIVER_CANCER_SUBCLASS_PROLIFERATION_UP & 118 & 1.57 & 0.046 \\
\hline NABA_COLLAGENS & 25 & 1.56 & 0.029 \\
\hline HALLMARK_IL2_STAT5_SIGNALING & 144 & 1.54 & 0.028 \\
\hline $\begin{array}{l}\text { HALLMARK_EPITHELIAL_MESENCHYMAL_TRANSITION } \\
\end{array}$ & 160 & 1.53 & 0.030 \\
\hline HALLMARK_KRAS_SIGNALING_UP & 159 & 1.51 & 0.017 \\
\hline
\end{tabular}

\begin{tabular}{|c|c|c|c|}
\hline \multicolumn{4}{|c|}{ STEM CLUSTER } \\
\hline \multicolumn{4}{|c|}{ Unsupervised GSEA } \\
\hline$\left(r^{2}\right)$ & SIZE & NES & NOM p-val \\
\hline REACTOME_INFLUENZA_LIFE_CYCLE & 79 & 2.16 & 0.0065 \\
\hline LEE_LIVER_CANCER_SURVIVAL_DN & 112 & 2.08 & 0 \\
\hline LE_EGR2_TARGETS_UP & 74 & 2.07 & 0 \\
\hline BENPORATH_ES_CORE_NINE_CORRELATED & 81 & 2.05 & 0 \\
\hline REACTOME_TRANSLATION & 77 & 2.04 & 0.0085 \\
\hline CHIANG_LIVER_CANCER_SUBCLASS_PROLIFERATION_UP & 118 & 2.00 & 0 \\
\hline LIAO_METASTASIS & 300 & 2.00 & 0 \\
\hline REACTOME_MITOTIC_M_M_G1_PHASES & 122 & 1.97 & 0.0083 \\
\hline CAIRO_HEPATOBLASTOMA_CLASSES_UP & 428 & 1.97 & 0.0063 \\
\hline RHODES_UNDIFFERENTIATED_CANCER & 53 & 1.95 & 0.0063 \\
\hline HALLMARK_MYC_TARGETS_V1 & 127 & 1.95 & 0.016 \\
\hline BOYAULT_LIVER_CANCER_SUBCLASS_G3_UP & 134 & 1.94 & 0.010 \\
\hline SARRIO_EPITHELIAL_MESENCHYMAL_TRANSITION_UP & 106 & 1.92 & 0.0062 \\
\hline WONG_EMBRYONIC_STEM_CELL_CORE & 229 & 1.92 & 0.0084 \\
\hline HALLMARK_G2M_CHECKPOINT & 149 & 1.91 & 0 \\
\hline REACTOME_CELL_CYCLE_MITOTIC & 209 & 1.91 & 0.0063 \\
\hline SHIPP_DLBCL_VS_FOLLICULAR_LYMPHOMA_UP & 27 & 1.90 & 0.0082 \\
\hline BHATTACHARYA_EMBRYONIC_STEM_CELL & 55 & 1.87 & 0 \\
\hline WHITFIELD_CELL_CYCLE_G2 & 90 & 1.86 & 0.0020 \\
\hline COULOUARN_TEMPORAL_TGFB1_SIGNATURE_UP & 71 & 1.85 & 0 \\
\hline HOLLEMAN_VINCRISTINE_RESISTANCE_B_ALL_UP & 28 & 1.85 & 0.0021 \\
\hline KOBAYASHI_EGFR_SIGNALING_24HR_DN & 186 & 1.85 & 0.0063 \\
\hline REACTOME_METABOLISM_OF_MRNA & 132 & 1.85 & 0.039 \\
\hline REACTOME_CELL_CYCLE & 261 & 1.85 & 0.0063 \\
\hline BASSO_B_LYMPHOCYTE_NETWORK & 96 & 1.85 & 0.0020 \\
\hline PID_MYC_ACTIV_PATHWAY & 55 & 1.83 & 0.0083 \\
\hline REACTOME_MITOTIC_G1_G1_S_PHASES & 92 & 1.83 & 0.013 \\
\hline CHEMNITZ_RESPONSE_TO_PROSTAGLANDIN_E2_UP & 90 & 1.82 & 0.011 \\
\hline ZAMORA_NOS2_TARGETS_UP & 45 & 1.82 & 0.011 \\
\hline BENPORATH_PROLIFERATION & 98 & 1.81 & 0.0062 \\
\hline REACTOME_SIGNALLING_TO_RAS & 18 & 1.81 & 0.0082 \\
\hline HALLMARK_MITOTIC_SPINDLE & 134 & 1.81 & 0.0040 \\
\hline TARTE_PLASMA_CELL_VS_PLASMABLAST_DN & 213 & 1.80 & 0.020 \\
\hline HALLMARK_MYC_TARGETS_V2 & 36 & 1.80 & 0.010 \\
\hline BILD_MYC_ONCOGENIC_SIGNATURE & 107 & 1.79 & 0 \\
\hline KEGG_SPLICEOSOME & 72 & 1.79 & 0.026 \\
\hline SCHLOSSER_MYC_TARGETS_AND_SERUM_RESPONSE_UP & 34 & 1.79 & 0.010 \\
\hline GRADE_COLON_AND_RECTAL_CANCER_UP & 182 & 1.78 & 0.012 \\
\hline WHITFIELD_CELL_CYCLE_G2_M & 144 & 1.78 & 0.010 \\
\hline BIOCARTA_CASPASE_PATHWAY & 21 & 1.77 & 0.004 \\
\hline BENPORATH_CYCLING_GENES & 412 & 1.77 & 0.0062 \\
\hline LEE_EARLY_T_LYMPHOCYTE_UP & 69 & 1.77 & 0.0041 \\
\hline \begin{tabular}{|c|} 
BOYAULT_LIVER_CANCER_SUBCLASS_G23_UP \\
\end{tabular} & 40 & 1.77 & 0.010 \\
\hline
\end{tabular}




\begin{tabular}{|c|c|c|c|}
\hline ROSTY_CERVICAL_CANCER_PROLIFERATION_CLUSTER & 110 & 1.76 & 0.0062 \\
\hline BIOCARTA_FAS_PATHWAY & 26 & 1.76 & 0.0082 \\
\hline BIDUS_METASTASIS_UP & 128 & 1.76 & 0.015 \\
\hline MORI_IMMATURE_B_LYMPHOCYTE_DN & 61 & 1.76 & 0.010 \\
\hline REACTOME_G1_S_TRANSITION & 78 & 1.75 & 0.027 \\
\hline SENGUPTA_NASOPHARYNGEAL_CARCINOMA_UP & 181 & 1.75 & 0.013 \\
\hline BORCZUK_MALIGNANT_MESOTHELIOMA_UP & 233 & 1.75 & 0.031 \\
\hline KEGG_CELL_CYCLE & 91 & 1.74 & 0.0085 \\
\hline BOYAULT_LIVER_CANCER_SUBCLASS_G123_UP & 32 & 1.74 & 0.010 \\
\hline ZHANG_BREAST_CANCER_PROGENITORS_UP & 241 & 1.73 & 0.014 \\
\hline PATIL_LIVER_CANCER & 458 & 1.73 & 0.026 \\
\hline HALLMARK_E2F_TARGETS & 140 & 1.72 & 0.016 \\
\hline RODRIGUES_THYROID_CARCINOMA_POORLY_DIFFERENTIATED_UP & 384 & 1.71 & 0.015 \\
\hline WOO_LIVER_CANCER_RECURRENCE_UP & 83 & 1.71 & 0 \\
\hline HOSHIDA_LIVER_CANCER_SUBCLASS_S1 & 192 & 1.71 & 0.021 \\
\hline ZHENG_GLIOBLASTOMA_PLASTICITY_UP & 168 & 1.70 & 0.0083 \\
\hline PARK_HSC_MARKERS & 27 & 1.70 & 0.022 \\
\hline WINNEPENNINCKX_MELANOMA_METASTASIS_UP & 94 & 1.70 & 0.033 \\
\hline KANG_DOXORUBICIN_RESISTANCE_UP & 42 & 1.70 & 0.0062 \\
\hline RAMASWAMY_METASTASIS_UP & 43 & 1.70 & 0.012 \\
\hline EGUCHI_CELL_CYCLE_RB1_TARGETS & 19 & 1.69 & 0.0020 \\
\hline CHIARADONNA_NEOPLASTIC_TRANSFORMATION_KRAS_UP & 93 & 1.69 & 0.0044 \\
\hline VANTVEER_BREAST_CANCER_POOR_PROGNOSIS & 34 & 1.69 & 0.0061 \\
\hline CAIRO_PML_TARGETS_BOUND_BY_MYC_UP & 16 & 1.69 & 0.024 \\
\hline CHICAS_RB1_TARGETS_GROWING & 165 & 1.68 & 0.011 \\
\hline YU_MYC_TARGETS_UP & 28 & 1.68 & 0.0043 \\
\hline REACTOME_SYNTHESIS_OF_DNA & 68 & 1.67 & 0.073 \\
\hline PID_P38_MK2_PATHWAY & 15 & 1.67 & 0.019 \\
\hline CHNG_MULTIPLE_MYELOMA_HYPERPLOID_UP & 29 & 1.67 & 0.045 \\
\hline LU_TUMOR_ANGIOGENESIS_UP & 21 & 1.66 & 0.011 \\
\hline STONER_ESOPHAGEAL_CARCINOGENESIS_UP & 23 & 1.64 & 0.025 \\
\hline ZHAN_MULTIPLE_MYELOMA_PR_UP & 26 & 1.64 & 0.0062 \\
\hline LI_WILMS_TUMOR_ANAPLASTIC_UP & 15 & 1.64 & 0.017 \\
\hline PRAMOONJAGO_SOX4_TARGETS_UP & 39 & 1.64 & 0.044 \\
\hline PID_AURORA_A_PATHWAY & 21 & 1.64 & 0.038 \\
\hline CAIRO_HEPATOBLASTOMA_UP & 146 & 1.63 & 0.034 \\
\hline LINDGREN_BLADDER_CANCER_CLUSTER_3_UP & 211 & 1.63 & 0.043 \\
\hline PID_PDGFRB_PATHWAY & 100 & 1.63 & 0.029 \\
\hline DANG_MYC_TARGETS_UP & 90 & 1.62 & 0.048 \\
\hline PID_RAC1_PATHWAY & 40 & 1.62 & 0.026 \\
\hline HOLLEMAN_VINCRISTINE_RESISTANCE_ALL_UP & 16 & 1.62 & 0.029 \\
\hline BIOCARTA_CELLCYCLE_PATHWAY & 18 & 1.62 & 0.011 \\
\hline WINTER_HYPOXIA_UP & 47 & 1.62 & 0.027 \\
\hline YAMASHITA_LIVER_CANCER_WITH_EPCAM_UP & 37 & 1.61 & 0.049 \\
\hline FEVR_CTNNB1_TARGETS_DN & 336 & 1.61 & 0.040 \\
\hline KAUFFMANN_MELANOMA_RELAPSE_UP & 44 & 1.61 & 0.043 \\
\hline WANG_TUMOR_INVASIVENESS_UP & 233 & 1.60 & 0.022 \\
\hline HALLMARK_MTORC1_SIGNALING & 160 & 1.51 & 0.048 \\
\hline
\end{tabular}




\section{Supporting Table S8. See Supplementary Data.docx file for caption.}

\begin{tabular}{|c|c|c|l|l|}
\hline \multicolumn{4}{|c|}{ OVERREPRESENTED ATTRIBUTES IN PERIVENOUS GENES } \\
\hline LOD & P_adj & attrib ID & Overrepresented GO terms \\
\hline 2.16 & 0.017 & GO:0016098 & monoterpenoid metabolic process \\
\hline 1.96 & 0.049 & GO:0061045 & negative regulation of wound healing \\
\hline 1.55 & 0.041 & GO:0008299 & isoprenoid biosynthetic process \\
\hline 1.52 & 0.001 & GO:0006695 & cholesterol biosynthetic process \\
\hline 1.45 & 0.001 & GO:0016126 & sterol biosynthetic process \\
\hline 1.40 & 0.012 & GO:0016709 & $\begin{array}{l}\text { oxidoreductase activity, acting on paired donors, with incorporation or reduction } \\
\text { oxygen }\end{array}$ \\
\hline 1.13 & 0.001 & GO:0046165 & alcohol biosynthetic process \\
\hline 1.11 & 0.002 & GO:0008203 & cholesterol metabolic process \\
\hline 1.10 & 0.002 & GO:0006694 & steroid biosynthetic process \\
\hline 1.06 & 0.005 & GO:0016125 & sterol metabolic process \\
\hline 1.04 & 0.031 & GO:0061041 & regulation of wound healing \\
\hline 1.01 & 0.043 & GO:0006720 & isoprenoid metabolic process \\
\hline 1.01 & 0.043 & GO:0042579 & microbody \\
\hline 1.00 & 0.012 & GO:0046906 & tetrapyrrole binding \\
\hline 0.97 & 0.019 & GO:1901617 & organic hydroxy compound biosynthetic process \\
\hline 0.97 & $<0.001$ & GO:0008202 & steroid metabolic process \\
\hline 0.81 & 0.001 & GO:0044283 & small molecule biosynthetic process \\
\hline 0.76 & 0.04 & GO:0006066 & alcohol metabolic process \\
\hline 0.69 & 0.014 & GO:0008610 & lipid biosynthetic process \\
\hline 0.60 & 0.001 & GO:0044711 & single-organism biosynthetic process \\
\hline 0.56 & 0.04 & GO:0044255 & cellular lipid metabolic process \\
\hline 0.54 & 0.011 & GO:0006629 & lipid metabolic process \\
\hline 0.49 & 0.001 & GO:0044281 & small molecule metabolic process \\
\hline 0.37 & 0.005 & GO:0044444 & cytoplasmic part \\
\hline 0.37 & 0.005 & GO:0044237 & cellular metabolic process \\
\hline 0.37 & 0.01 & GO:0044710 & single-organism metabolic process \\
\hline 0.37 & 0.04 & GO:0044699 & single-organism process \\
\hline 0.34 & 0.044 & GO:0008152 & metabolic process \\
\hline 0.34 & 0.04 & GO:0071704 & organic substance metabolic process \\
\hline & & & \\
\hline
\end{tabular}

\begin{tabular}{|c|c|c|c|c|}
\hline \multicolumn{3}{|c|}{ OVERREPRESENTED ATTRIBUTES IN PERIPORTAL GENES } \\
\hline LOD & P_adj & attrib ID & Overrepresented GO terms \\
\hline 3.06 & $<0.001$ & GO:0006572 & tyrosine catabolic process \\
\hline 2.86 & 0.001 & GO:0006116 & NADH oxidation \\
\hline 2.38 & 0.004 & GO:0008391 & arachidonic acid monooxygenase activity \\
\hline 2.38 & 0.004 & GO:0008392 & arachidonic acid epoxygenase activity \\
\hline 2.16 & 0.018 & GO:0016841 & ammonia-lyase activity \\
\hline 2.13 & 0.001 & GO:0005579 & membrane attack complex \\
\hline
\end{tabular}




\begin{tabular}{|c|c|c|c|}
\hline 2.11 & $<0.001$ & G0:0006558 & L-phenylalanine metabolic process \\
\hline 2.11 & $<0.001$ & GO:0006559 & L-phenylalanine catabolic process \\
\hline 2.11 & $<0.001$ & GO:0006570 & tyrosine metabolic process \\
\hline 2.11 & $<0.001$ & GO:1902221 & $\begin{array}{l}\text { erythrose 4-phosphate/phosphoenolpyruvate family amino acid metabolic } \\
\text { process }\end{array}$ \\
\hline 2.11 & $<0.001$ & GO:1902222 & $\begin{array}{l}\text { erythrose 4-phosphate/phosphoenolpyruvate family amino acid catabolic } \\
\text { process }\end{array}$ \\
\hline 2.09 & $<0.001$ & GO:0019373 & epoxygenase P450 pathway \\
\hline 1.93 & 0.001 & GO:0097267 & omega-hydroxylase P450 pathway \\
\hline 1.89 & $<0.001$ & G0:0019627 & urea metabolic process \\
\hline 1.83 & $<0.001$ & GO:0071941 & nitrogen cycle metabolic process \\
\hline 1.80 & $<0.001$ & G0:0009074 & aromatic amino acid family catabolic process \\
\hline 1.80 & 0.003 & GO:0000050 & urea cycle \\
\hline 1.67 & $<0.001$ & GO:0009072 & aromatic amino acid family metabolic process \\
\hline 1.65 & 0.013 & G0:0006525 & arginine metabolic process \\
\hline 1.65 & 0.013 & GO:0034185 & apolipoprotein binding \\
\hline 1.63 & $<0.001$ & GO:0034364 & high-density lipoprotein particle \\
\hline 1.60 & 0.001 & G0:0034308 & primary alcohol metabolic process \\
\hline 1.55 & $<0.001$ & G0:0017144 & drug metabolic process \\
\hline 1.54 & 0.041 & G0:0000097 & sulfur amino acid biosynthetic process \\
\hline 1.49 & $<0.001$ & GO:0034358 & plasma lipoprotein particle \\
\hline 1.46 & $<0.001$ & GO:0032994 & protein-lipid complex \\
\hline 1.45 & $<0.001$ & GO:1901606 & alpha-amino acid catabolic process \\
\hline 1.42 & $<0.001$ & GO:0009063 & cellular amino acid catabolic process \\
\hline 1.42 & $<0.001$ & GO:0000096 & sulfur amino acid metabolic process \\
\hline 1.39 & $<0.001$ & G0:0016054 & organic acid catabolic process \\
\hline 1.39 & $<0.001$ & G0:0046395 & carboxylic acid catabolic process \\
\hline 1.38 & $<0.001$ & G0:0006094 & gluconeogenesis \\
\hline 1.34 & $<0.001$ & GO:0046364 & monosaccharide biosynthetic process \\
\hline 1.34 & $<0.001$ & GO:0019319 & hexose biosynthetic process \\
\hline 1.34 & 0.024 & GO:0006730 & one-carbon metabolic process \\
\hline 1.31 & 0.001 & GO:0008206 & bile acid metabolic process \\
\hline 1.31 & 0.001 & GO:0009066 & aspartate family amino acid metabolic process \\
\hline 1.31 & $<0.001$ & GO:0051289 & protein homotetramerization \\
\hline 1.30 & 0.029 & GO:0009069 & serine family amino acid metabolic process \\
\hline 1.29 & $<0.001$ & G0:0072329 & monocarboxylic acid catabolic process \\
\hline 1.28 & $<0.001$ & GO:0044282 & small molecule catabolic process \\
\hline 1.26 & $<0.001$ & GO:0009064 & glutamine family amino acid metabolic process \\
\hline 1.26 & 0.047 & GO:0016709 & $\begin{array}{l}\text { oxidoreductase activity, acting on paired donors, with incorporation or reduction } \\
\text { of molecular oxygen, } \mathrm{NAD}(\mathrm{P}) \mathrm{H} \text { as one donor, and incorporation of one atom of } \\
\text { oxygen }\end{array}$ \\
\hline 1.25 & $<0.001$ & GO:1901605 & alpha-amino acid metabolic process \\
\hline 1.23 & 0.001 & GO:0030170 & pyridoxal phosphate binding \\
\hline 1.22 & $<0.001$ & GO:0019395 & fatty acid oxidation \\
\hline
\end{tabular}




\begin{tabular}{|c|c|c|c|}
\hline 1.21 & $<0.001$ & G0:0009062 & fatty acid catabolic process \\
\hline 1.21 & $<0.001$ & GO:0034440 & lipid oxidation \\
\hline 1.21 & $<0.001$ & GO:0006805 & xenobiotic metabolic process \\
\hline 1.21 & $<0.001$ & GO:0043648 & dicarboxylic acid metabolic process \\
\hline 1.20 & $<0.001$ & GO:1901607 & alpha-amino acid biosynthetic process \\
\hline 1.17 & $<0.001$ & G0:0016597 & amino acid binding \\
\hline 1.15 & $<0.001$ & GO:0008652 & cellular amino acid biosynthetic process \\
\hline 1.15 & 0.026 & GO:0016627 & oxidoreductase activity, acting on the $\mathrm{CH}-\mathrm{CH}$ group of donors \\
\hline 1.15 & 0.026 & GO:0019369 & arachidonic acid metabolic process \\
\hline 1.13 & 0.03 & GO:0042632 & cholesterol homeostasis \\
\hline 1.12 & $<0.001$ & GO:0001676 & long-chain fatty acid metabolic process \\
\hline 1.12 & 0.038 & GO:0055092 & sterol homeostasis \\
\hline 1.12 & $<0.001$ & GO:0019752 & carboxylic acid metabolic process \\
\hline 1.11 & 0.041 & GO:1901616 & organic hydroxy compound catabolic process \\
\hline 1.11 & $<0.001$ & GO:0051262 & protein tetramerization \\
\hline 1.11 & $<0.001$ & GO:0032787 & monocarboxylic acid metabolic process \\
\hline 1.10 & $<0.001$ & G0:0016614 & oxidoreductase activity, acting on $\mathrm{CH}-\mathrm{OH}$ group of donors \\
\hline 1.09 & 0.001 & GO:0004497 & monooxygenase activity \\
\hline 1.09 & $<0.001$ & GO:0072562 & blood microparticle \\
\hline 1.08 & 0.011 & GO:0072376 & protein activation cascade \\
\hline 1.07 & 0.013 & GO:0005319 & lipid transporter activity \\
\hline 1.05 & $<0.001$ & GO:0006082 & organic acid metabolic process \\
\hline 1.05 & $<0.001$ & GO:0043436 & oxoacid metabolic process \\
\hline 1.05 & $<0.001$ & G0:0006732 & coenzyme metabolic process \\
\hline 1.03 & $<0.001$ & G0:0016051 & carbohydrate biosynthetic process \\
\hline 1.03 & $<0.001$ & GO:0016616 & $\begin{array}{l}\text { oxidoreductase activity, acting on the } \mathrm{CH}-\mathrm{OH} \text { group of donors, NAD or NADP as } \\
\text { acceptor }\end{array}$ \\
\hline 1.02 & $<0.001$ & GO:0006520 & cellular amino acid metabolic process \\
\hline 1.02 & $<0.001$ & GO:0048037 & cofactor binding \\
\hline 1.02 & $<0.001$ & GO:0006631 & fatty acid metabolic process \\
\hline 1.02 & 0.004 & GO:0033559 & unsaturated fatty acid metabolic process \\
\hline 1.02 & $<0.001$ & GO:0006575 & cellular modified amino acid metabolic process \\
\hline 1.00 & 0.041 & GO:0006641 & triglyceride metabolic process \\
\hline 1.00 & $<0.001$ & GO:0016053 & organic acid biosynthetic process \\
\hline 1.00 & $<0.001$ & GO:0046394 & carboxylic acid biosynthetic process \\
\hline 0.99 & 0.007 & GO:0009108 & coenzyme biosynthetic process \\
\hline 0.99 & $<0.001$ & GO:0031406 & carboxylic acid binding \\
\hline 0.99 & $<0.001$ & GO:0043177 & organic acid binding \\
\hline 0.99 & 0.001 & G0:0030258 & lipid modification \\
\hline 0.98 & $<0.001$ & GO:0050662 & coenzyme binding \\
\hline 0.98 & $<0.001$ & GO:0051186 & cofactor metabolic process \\
\hline 0.98 & 0.05 & GO:0006639 & acylglycerol metabolic process \\
\hline 0.97 & 0.05 & GO:0006638 & neutral lipid metabolic process \\
\hline 0.97 & 0.05 & G0:0055088 & lipid homeostasis \\
\hline
\end{tabular}




\begin{tabular}{|c|c|c|c|}
\hline 0.96 & $<0.001$ & G0:0006006 & glucose metabolic process \\
\hline 0.95 & 0.001 & G0:0043603 & cellular amide metabolic process \\
\hline 0.94 & $<0.001$ & G0:0044283 & small molecule biosynthetic process \\
\hline 0.94 & 0.005 & GO:0020037 & heme binding \\
\hline 0.93 & 0.007 & GO:0044242 & cellular lipid catabolic process \\
\hline 0.93 & 0.007 & GO:0051188 & cofactor biosynthetic process \\
\hline 0.93 & 0.029 & GO:0042579 & microbody \\
\hline 0.92 & 0.001 & GO:0016829 & lyase activity \\
\hline 0.91 & 0.011 & GO:0046906 & tetrapyrrole binding \\
\hline 0.91 & $<0.001$ & GO:0005759 & mitochondrial matrix \\
\hline 0.89 & $<0.001$ & G0:0016491 & oxidoreductase activity \\
\hline 0.89 & 0.003 & GO:0016705 & $\begin{array}{l}\text { oxidoreductase activity, acting on paired donors, with incorporation or reduction } \\
\text { of molecular oxygen }\end{array}$ \\
\hline 0.86 & $<0.001$ & GO:0051260 & protein homooligomerization \\
\hline 0.86 & 0.001 & GO:0019318 & hexose metabolic process \\
\hline 0.85 & $<0.001$ & GO:0008202 & steroid metabolic process \\
\hline 0.84 & $<0.001$ & GO:0055114 & oxidation-reduction process \\
\hline 0.84 & $<0.001$ & GO:0044281 & small molecule metabolic process \\
\hline 0.82 & 0.019 & GO:0006869 & lipid transport \\
\hline 0.82 & 0.001 & GO:0005996 & monosaccharide metabolic process \\
\hline 0.80 & 0.001 & GO:0006790 & sulfur compound metabolic process \\
\hline 0.76 & 0.025 & GO:0016042 & lipid catabolic process \\
\hline 0.75 & $<0.001$ & GO:0006629 & lipid metabolic process \\
\hline 0.75 & $<0.001$ & GO:0044255 & cellular lipid metabolic process \\
\hline 0.75 & $<0.001$ & GO:0044710 & single-organism metabolic process \\
\hline 0.73 & $<0.001$ & GO:0044712 & single-organism catabolic process \\
\hline 0.72 & 0.001 & GO:0051259 & protein oligomerization \\
\hline 0.70 & $<0.001$ & GO:0043233 & organelle lumen \\
\hline 0.69 & 0.001 & GO:1901615 & organic hydroxy compound metabolic process \\
\hline 0.69 & 0.025 & GO:0015711 & organic anion transport \\
\hline 0.69 & $<0.001$ & GO:0044711 & single-organism biosynthetic process \\
\hline 0.67 & $<0.001$ & GO:1901565 & organonitrogen compound catabolic process \\
\hline 0.67 & $<0.001$ & GO:0070013 & intracellular organelle lumen \\
\hline 0.66 & $<0.001$ & GO:0031974 & membrane-enclosed lumen \\
\hline 0.66 & $<0.001$ & GO:1901564 & organonitrogen compound metabolic process \\
\hline 0.65 & $<0.001$ & GO:1901566 & organonitrogen compound biosynthetic process \\
\hline 0.65 & 0.005 & GO:0006820 & anion transport \\
\hline 0.61 & $<0.001$ & GO:0048878 & chemical homeostasis \\
\hline 0.61 & $<0.001$ & GO:0044248 & cellular catabolic process \\
\hline 0.57 & $<0.001$ & GO:0009056 & catabolic process \\
\hline 0.57 & $<0.001$ & GO:1901575 & organic substance catabolic process \\
\hline 0.56 & $<0.001$ & GO:0044699 & single-organism process \\
\hline 0.55 & 0.009 & GO:0014070 & response to organic cyclic compound \\
\hline 0.55 & $<0.001$ & GO:0003824 & catalytic activity \\
\hline
\end{tabular}




\begin{tabular}{|l|l|l|l|}
\hline 0.52 & $<0.001$ & $G 0: 0043230$ & extracellular organelle \\
\hline 0.52 & $<0.001$ & $G 0: 0065010$ & extracellular membrane-bounded organelle \\
\hline 0.52 & $<0.001$ & $G 0: 0070062$ & extracellular vesicular exosome \\
\hline 0.51 & 0.018 & $\mathrm{GO}: 0044429$ & mitochondrial part \\
\hline 0.51 & 0.001 & $\mathrm{GO}: 0042592$ & homeostatic process \\
\hline 0.50 & $<0.001$ & $\mathrm{GO}: 0008152$ & metabolic process \\
\hline 0.48 & 0.024 & $\mathrm{GO}: 1901361$ & organic cyclic compound catabolic process \\
\hline 0.48 & $<0.001$ & $\mathrm{GO}: 0044421$ & extracellular region part \\
\hline 0.45 & $<0.001$ & $\mathrm{GO}: 0031988$ & membrane-bounded vesicle \\
\hline 0.45 & $<0.001$ & $\mathrm{GO}: 0031982$ & vesicle \\
\hline 0.43 & $<0.001$ & $\mathrm{GO}: 0071704$ & organic substance metabolic process \\
\hline 0.43 & $<0.001$ & $\mathrm{GO}: 0043168$ & anion binding \\
\hline 0.40 & $<0.001$ & $\mathrm{GO}: 0044238$ & primary metabolic process \\
\hline 0.39 & $<0.001$ & $\mathrm{GO}: 0044444$ & cytoplasmic part \\
\hline 0.39 & 0.001 & $\mathrm{GO}: 0036094$ & small molecule binding \\
\hline 0.38 & 0.001 & $\mathrm{GO}: 0065008$ & regulation of biological quality \\
\hline 0.37 & $<0.001$ & $\mathrm{GO}: 0044237$ & cellular metabolic process \\
\hline 0.33 & 0.001 & $\mathrm{GO}: 0044763$ & single-organism cellular process \\
\hline
\end{tabular}

\begin{tabular}{|c|c|c|c|}
\hline \multicolumn{4}{|r|}{ OVERREPRESENTED ATTRIBUTES IN STEM GENES } \\
\hline LOD & P_adj & attrib ID & Overrepresented GO terms \\
\hline 2.22 & 0.036 & G0:0000778 & condensed nuclear chromosome kinetochore \\
\hline 1.69 & 0.025 & G0:0000779 & condensed chromosome, centromeric region \\
\hline 1.63 & 0.042 & G0:0005583 & fibrillar collagen \\
\hline 1.17 & 0.042 & G0:0030834 & regulation of actin filament depolymerization \\
\hline 1.05 & 0.004 & G0:1901879 & regulation of protein depolymerization \\
\hline 0.96 & 0.027 & G0:0043244 & regulation of protein complex disassembly \\
\hline 0.86 & 0.044 & GO:0008064 & regulation of actin polymerization or depolymerization \\
\hline 0.85 & 0.05 & G0:0030832 & regulation of actin filament length \\
\hline 0.82 & 0.01 & GO:0005819 & spindle \\
\hline 0.76 & $<0.001$ & G0:0030335 & positive regulation of cell migration \\
\hline 0.75 & $<0.001$ & GO:2000147 & positive regulation of cell motility \\
\hline 0.74 & $<0.001$ & G0:0051272 & positive regulation of cellular component movement \\
\hline 0.72 & $<0.001$ & G0:0040017 & positive regulation of locomotion \\
\hline 0.71 & $<0.001$ & GO:0051493 & regulation of cytoskeleton organization \\
\hline 0.70 & $<0.001$ & G0:0030198 & extracellular matrix organization \\
\hline 0.70 & $<0.001$ & G0:0043062 & extracellular structure organization \\
\hline 0.69 & 0.029 & GO:0050900 & leukocyte migration \\
\hline 0.68 & $<0.001$ & GO:0022411 & cellular component disassembly \\
\hline 0.66 & $<0.001$ & G0:0007599 & hemostasis \\
\hline 0.65 & $<0.001$ & G0:0007017 & microtubule-based process \\
\hline 0.65 & $<0.001$ & G0:0007596 & blood coagulation \\
\hline 0.65 & $<0.001$ & G0:0050817 & coagulation \\
\hline 0.61 & $<0.001$ & G0:0050878 & regulation of body fluid levels \\
\hline
\end{tabular}




\begin{tabular}{|c|c|c|c|}
\hline 0.59 & 0.002 & GO:0000278 & mitotic cell cycle \\
\hline 0.59 & $<0.001$ & GO:0030334 & regulation of cell migration \\
\hline 0.56 & $<0.001$ & GO:2000145 & regulation of cell motility \\
\hline 0.56 & $<0.001$ & GO:0033043 & regulation of organelle organization \\
\hline 0.56 & $<0.001$ & GO:0016477 & cell migration \\
\hline 0.55 & 0.036 & G0:0051129 & negative regulation of cellular component organization \\
\hline 0.54 & 0.001 & GO:0040012 & regulation of locomotion \\
\hline 0.53 & $<0.001$ & GO:0008092 & cytoskeletal protein binding \\
\hline 0.53 & 0.008 & GO:0051270 & regulation of cellular component movement \\
\hline 0.52 & $<0.001$ & GO:0005856 & cytoskeleton \\
\hline 0.52 & $<0.001$ & GO:0006928 & cellular component movement \\
\hline 0.52 & $<0.001$ & GO:0048870 & cell motility \\
\hline 0.50 & $<0.001$ & GO:0044699 & single-organism process \\
\hline 0.50 & 0.003 & GO:0032403 & protein complex binding \\
\hline 0.50 & 0.001 & GO:0022603 & regulation of anatomical structure morphogenesis \\
\hline 0.49 & $<0.001$ & GO:0043230 & extracellular organelle \\
\hline 0.49 & $<0.001$ & GO:0065010 & extracellular membrane-bounded organelle \\
\hline 0.49 & $<0.001$ & GO:0070062 & extracellular vesicular exosome \\
\hline 0.48 & $<0.001$ & GO:0031988 & membrane-bounded vesicle \\
\hline 0.47 & $<0.001$ & GO:0031982 & vesicle \\
\hline 0.46 & $<0.001$ & GO:0022402 & cell cycle process \\
\hline 0.46 & $<0.001$ & GO:0051128 & regulation of cellular component organization \\
\hline 0.46 & $<0.001$ & GO:0044430 & cytoskeletal part \\
\hline 0.45 & $<0.001$ & GO:0005829 & cytosol \\
\hline 0.45 & 0.044 & GO:0008284 & positive regulation of cell proliferation \\
\hline 0.45 & 0.02 & G0:0040011 & locomotion \\
\hline 0.45 & $<0.001$ & G0:0016043 & cellular component organization \\
\hline 0.44 & $<0.001$ & GO:0044421 & extracellular region part \\
\hline 0.44 & $<0.001$ & GO:0071840 & cellular component organization or biogenesis \\
\hline 0.44 & $<0.001$ & G0:0044763 & single-organism cellular process \\
\hline 0.43 & $<0.001$ & GO:0043228 & non-membrane-bounded organelle \\
\hline 0.43 & $<0.001$ & GO:0043232 & intracellular non-membrane-bounded organelle \\
\hline 0.43 & $<0.001$ & GO:0042981 & regulation of apoptotic process \\
\hline 0.43 & $<0.001$ & GO:0043067 & regulation of programmed cell death \\
\hline 0.42 & $<0.001$ & GO:0010941 & regulation of cell death \\
\hline 0.41 & 0.008 & GO:0005615 & extracellular space \\
\hline 0.41 & $<0.001$ & GO:0065008 & regulation of biological quality \\
\hline 0.41 & $<0.001$ & G0:0042127 & regulation of cell proliferation \\
\hline 0.40 & $<0.001$ & GO:0048522 & positive regulation of cellular process \\
\hline 0.39 & $<0.001$ & GO:0048518 & positive regulation of biological process \\
\hline 0.38 & $<0.001$ & GO:0005515 & protein binding \\
\hline 0.38 & $<0.001$ & G0:0044424 & intracellular part \\
\hline 0.38 & 0.044 & GO:0071822 & protein complex subunit organization \\
\hline 0.36 & $<0.001$ & GO:0048523 & negative regulation of cellular process \\
\hline
\end{tabular}




\begin{tabular}{|c|c|c|c|}
\hline 0.35 & $<0.001$ & GO:0048519 & negative regulation of biological process \\
\hline 0.35 & 0.025 & GO:0002376 & immune system process \\
\hline 0.34 & $<0.001$ & GO:0005737 & cytoplasm \\
\hline 0.33 & 0.042 & GO:0032879 & regulation of localization \\
\hline 0.32 & 0.014 & GO:0010033 & response to organic substance \\
\hline 0.32 & 0.014 & GO:0009987 & cellular process \\
\hline 0.31 & $<0.001$ & GO:0043226 & organelle \\
\hline 0.31 & $<0.001$ & GO:0065007 & biological regulation \\
\hline 0.30 & $<0.001$ & GO:0044444 & cytoplasmic part \\
\hline 0.28 & $<0.001$ & GO:0043227 & membrane-bounded organelle \\
\hline 0.27 & 0.001 & G0:0050794 & regulation of cellular process \\
\hline 0.25 & 0.014 & GO:0050789 & regulation of biological process \\
\hline
\end{tabular}




\section{Supporting Table S9. See Supplementary Data.docx file for caption.}

(a) clusterinal

\begin{tabular}{|l|l|l|l|}
\hline Gene symbol & Gene clustering \\
\hline
\end{tabular} \begin{tabular}{|l|l|}
\hline CYP2E1 & PERIVENOUS \\
\hline
\end{tabular} \begin{tabular}{|l|l}
\hline AOX1 & PERIVENOUS \\
\hline
\end{tabular} \begin{tabular}{|l|l}
\hline SPCS1 & PERIVENOUS \\
\hline
\end{tabular} GLUL (chin GGH ATOX1 CERS2 TAX1BP1 EFR3A HMGCS1 CEBPA PPAP2A DSP AP3S1 APSTT MPC2 CADM1 \begin{tabular}{l} 
IARS2 \\
\hline CYDMA \\
\hline
\end{tabular} CYP3A4 SMYD2 \begin{tabular}{ll}
\hline LBR \\
\hline MRPL24 \\
\hline
\end{tabular}

MRPL24 FAT1 PRPSAP1

\begin{tabular}{|c|c|c|c|c|c|}
\hline & \multicolumn{4}{|c|}{ [Low 95\% Cl - Mean - High 95\% Cl] } & \multirow[b]{2}{*}{$P=$} \\
\hline Gene description & Cluster PP & Cluster PV & Cluster ECM & Cluster STEM & \\
\hline chrome P450, family 2, subfamily E, polypeptide 1 & {$[12.31-12.53-12.75]$} & {$[14.52-14.62-14.73]$} & {$[12.9-13.1-13.29]$} & {$[11.43-11.73-12.04]$} & $6.33 \mathrm{E}-23$ \\
\hline lehyde oxidase 1 & {$[11.75-11.91-12.06]$} & {$[13.21-13.28-13.35]$} & {$[11.7-11.86-12.01]$} & {$[10.58-10.78-10.99]$} & $7.43 \mathrm{E}-92$ \\
\hline ginal peptidase complex subunit 1 homolog (S. cerevisiae) & {$[12.69-12.72-12.76]$} & {$[12.93-12.98-13.03]$} & {$[12.63-12.68-12.73]$} & {$[12.69-12.74-12.79]$} & $2.62 \mathrm{E}-58$ \\
\hline lutamate-ammonia ligase & {$[9.59-9.7-9.81]$} & {$[12.71-12.83-12.96]$} & {$[9.59-9.7-9.81]$} & {$[9.47-9.58-9.7]$} & $8.39 \mathrm{E}-15$ \\
\hline $\begin{array}{l}\text { amma-glutamyl hydrolase (conjugase, folylpolygammaglutamyl } \\
\text { ydrolase) }\end{array}$ & {$[11.96-12.02-12.09]$} & [ $12.72-12.82-12.92$ ] & {$[11.9-12-12.09]$} & [ $11.53-11.65-11.77$ ] & $5.72 \mathrm{E}-21$ \\
\hline TX1 antioxidant protein 1 homolog (yeast) & {$[12.34-12.39-12.44]$} & {$[12.61-12.69-12.76]$} & {$[12.19-12.26-12.32]$} & {$[11.75-11.81-11.88]$} & $4.06 \mathrm{E}-43$ \\
\hline AG1 homolog, ceramide synthase 2 & {$[12.08-12.12-12.16]$} & {$[12.22-12.28-12.34]$} & {$[12.07-12.12-12.17]$} & {$[11.82-11.9-11.98]$} & $5.68 \mathrm{E}-07$ \\
\hline ax1 (human T-cell leukemia virus type I) binding protein 1 & {$[11.97-12-12.04]$} & {$[12.19-12.24-12.3]$} & {$[11.92-11.97-12.01]$} & {$[11.93-11.99-12.04]$} & $1.05 \mathrm{E}-14$ \\
\hline FR3 homolog A (S. cerevisiae) & {$[11.68-11.73-11.78]$} & {$[12.12-12.18-12.25]$} & {$[11.8-11.86-11.92]$} & {$[11.58-11.65-11.71]$} & $6.13 \mathrm{E}-74$ \\
\hline -hydroxy-3-methylglutaryl-CoA synthase 1 (soluble) & {$[11.8-11.89-11.99]$} & {$[11.93-12.02-12.12]$} & {$[11.91-11.99-12.08]$} & {$[11.72-11.85-11.97]$} & $9.29 \mathrm{E}-65$ \\
\hline CAAT/enhancer binding protein (C/EBP), alpha & {$[11.89-11.97-12.05]$} & {$[11.84-11.94-12.04]$} & {$[12.16-12.24-12.31]$} & {$[11.84-11.99-12.14]$} & $1.52 \mathrm{E}-58$ \\
\hline hosphatidic acid phosphatase type $2 \mathrm{~A}$ & {$[11.29-11.34-11.4]$} & {$[11.75-11.84-11.93]$} & {$[11.33-11.4-11.47]$} & {$[10.81-10.9-10.99]$} & $6.25 \mathrm{E}-101$ \\
\hline esmoplakin & {$[11.26-11.31-11.36]$} & {$[11.77-11.84-11.9]$} & {$[11.37-11.42-11.48]$} & {$[11.49-11.58-11.67]$} & $4.41 \mathrm{E}-63$ \\
\hline daptor-related protein complex 3 , sigma 1 subunit & {$[11.45-11.49-11.53]$} & {$[11.75-11.81-11.88]$} & {$[11.57-11.61-11.65]$} & {$[11.53-11.59-11.65]$} & $6.13 \mathrm{E}-03$ \\
\hline lyceronephosphate O-acyltransferase & {$[11.11-11.15-11.2]$} & {$[11.71-11.77-11.83]$} & {$[11.38-11.43-11.48]$} & {$[11.48-11.55-11.62]$} & $1.02 \mathrm{E}-33$ \\
\hline itochondrial pyruvate carrier 2 & {$[11.67-11.72-11.78]$} & {$[11.62-11.68-11.75]$} & {$[11.66-11.73-11.8]$} & {$[11.43-11.53-11.62]$} & $1.03 \mathrm{E}-39$ \\
\hline ell adhesion molecule 1 & {$[11.22-11.3-11.38]$} & {$[11.56-11.66-11.76]$} & {$[11.04-11.14-11.24]$} & {$[10.24-10.38-10.52]$} & $5.59 \mathrm{E}-18$ \\
\hline oleucyl-tRNA synthetase 2 , mit & {$[11.28-11.33-11.38]$} & {$[11.59-11.65-11.72]$} & {$[11.37-11.42-11.48]$} & {$[11.38-11.45-11.52]$} & $1.17 \mathrm{E}-20$ \\
\hline tochrome P45 & {$[10.2-10.35-10.51]$} & {$[11.47-11.64-11.81]$} & {$[9.25-9.4-9.56]$} & {$[8-8.17-8.34]$} & $1.40 \mathrm{E}-101$ \\
\hline ET and MYND domain containing 2 & {$[10.41-10.47-10.54]$} & {$[11.48-11.56-11.65]$} & {$[10.54-10.6-10.67]$} & {$[10.7-10.77-10.84]$} & $4.22 \mathrm{E}-43$ \\
\hline min B receptor & {$[11.04-11.1-11.17]$} & {$[11.39-11.47-11.54]$} & {$[11.48-11.55-11.61]$} & {$[11.74-11.82-11.91]$} & $1.31 \mathrm{E}-25$ \\
\hline itochondrial ribosomal protein L24 & {$[11.01-11.07-11.12]$} & {$[11.3-11.37-11.45]$} & {$[11.04-11.11-11.17]$} & {$[10.86-10.94-11.03]$} & $2.19 \mathrm{E}-01$ \\
\hline AT tumor suppressor homolog 1 (Drosophila) & {$[10.9-10.97-11.04]$} & {$[11.2-11.3-11.4]$} & {$[10.7-10.78-10.87]$} & {$[10.61-10.7-10.79]$} & $1.82 \mathrm{E}-01$ \\
\hline hosphoribosyl pyrophosphate synthetase-associated protein 1 & {$[11.1-11.15-11.2]$} & {$[11.18-11.25-11.31]$} & {$[11.05-11.11-11.17]$} & {$[10.92-10.98-11.04]$} & $1.46 \mathrm{E}-13$ \\
\hline rnithine aminotransferase & {$[9.63-9.77-9.91]$} & {$[10.97-11.18-11.39]$} & {$[10.1-10.25-10.41]$} & {$[10.33-10.5-10.67]$} & $3.16 \mathrm{E}-01$ \\
\hline ytochrome P450, family 51 , su & {$[11.1-11.16-11.23]$} & {$[11.1-11.18-11.25]$} & {$[11.07-11.14-11.2]$} & {$[10.87-10.96-11.05]$} & $9.97 \mathrm{E}-78$ \\
\hline accharopine dehydrogenase (putative) & {$[11.38-11.45-11.52]$} & {$[11.05-11.16-11.26]$} & {$[11.3-11.38-11.47]$} & {$[10.76-10.87-10.98]$} & $2.16 \mathrm{E}-42$ \\
\hline orting and assembly machinery component 50 homolog (S. cerevisiae) & {$[10.5-10.56-10.62]$} & [ $11.02-11.1-11.17]$ & [ $10.58-10.64-10.7$ ] & [ $10.56-10.63-10.7]$ & 2.84E-30 \\
\hline generating islet-derived 3 alpha & {$[6.93-7.17-7.4]$} & {$[10.64-11.03-11.42]$} & {$[7.18-7.43-7.69]$} & {$[7.56-7.89-8.21]$} & $6.38 \mathrm{E}-36$ \\
\hline istone cluster $1, \mathrm{H} 2 \mathrm{ac}$ & {$[10.36-10.46-10.56]$} & {$[10.91-11.03-11.14]$} & $\frac{1}{[10.27-10.38-10.48]}$ & {$[10.5-10.62-10.74]$} & $2.79 \mathrm{E}-51$ \\
\hline AB4A, member RAS oncogene family & {$[10.47-10.52-10.57]$} & {$[10.96-11.02-11.09]$} & {$[10.47-10.53-10.6]$} & {$[10.35-10.43-10.51]$} & $4.42 \mathrm{E}-25$ \\
\hline opentenyl-diphosphate delta isomerase 1 & {$[10.81-10.88-10.96]$} & {$[10.89-10.98-11.06]$} & {$[10.86-10.94-11.02]$} & {$[10.73-10.83-10.93]$} & $5.72 \mathrm{E}-30$ \\
\hline TPase, $\mathrm{H}+$ transporting, lysosomal V0 subunit a1 & {$[10.59-10.64-10.69]$} & {$[10.89-10.95-11.01]$} & {$[10.69-10.74-10.79]$} & {$[10.54-10.6-10.67]$} & $4.46 \mathrm{E}-50$ \\
\hline ho-associated, coiled-coil containing protein kinase 2 & {$[10.21-10.26-10.31]$} & {$[10.88-10.95-11.01]$} & {$[10.35-10.4-10.46$ ] } & {$[10.43-10.5-10.56$ ] } & $5.20 \mathrm{E}-28$ \\
\hline eoxyuridine triphosphatase & {$[10.21-10.27-10.33]$} & {$[10.84-10.93-11.02]$} & {$[10.12-10.19-10.25]$} & {$[10.1-10.19-10.28$ ] } & $2.18 \mathrm{E}-51$ \\
\hline
\end{tabular}

Hepatology

This article is protected by copyright. All rights reserved. 


\begin{tabular}{|c|c|c|c|c|c|c|c|}
\hline PREB & PERIVENOUS & prolactin regulatory element binding & {$[10.1-10.14-10.19]$} & {$[10.8-10.88-10.96]$} & {$[10.1-10.15-10.2]$} & {$[10.04-10.1-10.16]$} & $9.99 \mathrm{E}-23$ \\
\hline |LPPR1 & PERIVENOUS & lipid phosphate phosphatase-related protein type 1 & {$[9.03-9.12-9.21]$} & {$[10.76-10.87-10.99]$} & {$[9.21-9.32-9.44]$} & {$[9.15-9.34-9.53$ ] } & 3.09E-24 \\
\hline TBCE & PERIVENOUS & tubulin folding cofactor $\mathrm{E}$ & {$[10.19-10.25-10.3]$} & {$[10.76-10.82-10.88]$} & [ 10.48 - 10.54 - 10.61 ] & [ $10.39-10.47-10.55]$ & $4.12 \mathrm{E}-17$ \\
\hline CD36 & PERIVENOUS & CD36 molecule (thrombospondin receptor) & {$[9.7-9.78-9.87$ ] } & {$[10.62-10.75-10.89]$} & {$[9.29-9.4-9.52$ ] } & {$[9.29-9.44-9.6$ ] } & $1.71 \mathrm{E}-06$ \\
\hline PIR & PERIVENOUS & pirin (iron-binding nuclear protein) & {$[9.97-10.05-10.13]$} & {$[10.61-10.71-10.81]$} & {$[9.87-9.98-10.08]$} & {$[9.62-9.76-9.9]$} & $7.57 \mathrm{E}-45$ \\
\hline SLCO1B3 & PERIVENOUS & solute carrier organic anion transporter family, member $1 \mathrm{~B} 3$ & {$[7.55-7.79-8.03]$} & {$[10.37-10.7-11.02]$} & {$[8.07-8.33-8.58]$} & {$[7.89-8.15-8.41]$} & $1.54 \mathrm{E}-30$ \\
\hline TKT & PERIVENOUS & transketolase & {$[9.87-9.97-10.06]$} & {$[10.53-10.67-10.8]$} & {$[10.26-10.37-10.47]$} & {$[10.64-10.75-10.86]$} & $7.76 \mathrm{E}-30$ \\
\hline SYF2 & PERIVENOUS & SYF2 homolog, RNA splicing factor (S. cerevisiae) & $\mid[10.28-10.33-10.37]$ & {$[$ [ $10.61-10.66-10.71]$} & {$[10.27-10.32-10.38]$} & {$[10.28-10.34-10.4]$} & $2.43 \mathrm{E}-21$ \\
\hline PEX19 & PERIVENOUS & peroxisomal biogenesis factor 19 & {$[$ [ $10.42-10.47-10.52]$} & [ $10.58-10.64-10.71]$ & {$[10.22-10.29-10.35]$} & {$[9.9-9.98-10.06]$} & $9.67 \mathrm{E}-31$ \\
\hline MCUR1 & PERIVENOUS & mitochondrial calcium uniporter regulator 1 & {$[10.23-10.28-10.33]$} & {$[10.54-10.63-10.72]$} & {$[10.03-10.1-10.16]$} & {$[9.97-10.05-10.12$ ] } & $5.84 \mathrm{E}-30$ \\
\hline PMVKK & PERIVENOUS & phosphomevalonate kinase & {$[10.34-10.39-10.45]$} & {$[10.51-10.58-10.66$ ] } & {$[10.26-10.32-10.38$ ] } & {$[10.02-10.1-10.17]$} & $2.96 \mathrm{E}-20$ \\
\hline ELOVL2 & PERIVENOUS & $\begin{array}{l}\text { elongation of very long chain fatty acids (FEN1/Elo2, SUR4/Elo3, yeast)- } \\
\text { like } 2\end{array}$ & {$[10.17-10.25-10.33]$} & {$[10.4-10.54-10.68]$} & {$[10.23-10.32-10.42]$} & {$[9.89-10.04-10.19]$} & $2.16 \mathrm{E}-26$ \\
\hline PBXIP1 & PERIVENOUS & pre-B-cell leukemia homeobox interacting protein 1 & {$[10.05-10.11-10.16]$} & {$[10.43-10.5-10.58]$} & [ $10.11-10.17-10.23$ ] & {$[10.12-10.19-10.27]$} & 1.37E-17 \\
\hline LGALS8 & PERIVENOUS & lectin, galactoside-binding, soluble, 8 & [ $9.84-9.9-9.96$ ] & {$[10.43-10.5-10.57]$} & [ $10.01-10.08-10.15]$ & {$[9.83-9.91-10]$} & $5.49 \mathrm{E}-23$ \\
\hline TTC1 & PERIVENOUS & tetratricopeptide repeat domain 1 & {$[10.27-10.31-10.35]$} & {$[10.44-10.5-10.55]$} & {$[10.28-10.32-10.36$ ] } & {$[10.1-10.15-10.2$ ] } & $1.89 \mathrm{E}-16$ \\
\hline CUL1 & PERIVENOUS & cullin 1 & {$[10.13-10.18-10.23]$} & {$[10.4-10.48-10.55]$} & {$[10.07-10.13-10.2$ ] } & {$[9.99-10.06-10.13]$} & $9.99 \mathrm{E}-15$ \\
\hline LANCL1 & PERIVENOUS & LanC lantibiotic synthetase component C-like 1 (bacterial) & {$[10.3-10.35-10.41]$} & {$[10.4-10.48-10.55]$} & {$[10.39-10.45-10.52]$} & {$[10.23-10.31-10.38]$} & $8.13 \mathrm{E}-14$ \\
\hline KLHL21 & PERIVENOUS & kelch-like 21 (Drosophila) & {$[9.83-9.9-9.97$ ] } & {$[10.38-10.47-10.55]$} & [ $9.81-9.89-9.97$ ] & {$[9.8-9.89-9.98]$} & $3.84 \mathrm{E}-46$ \\
\hline HMGCR & PERIVENOUS & 3-hydroxy-3-methylglutaryl-CoA reductase & {$[10.35-10.42-10.5]$} & {$[10.37-10.46-10.55]$} & [ $10.43-10.51-10.59]$ & {$[10.39-10.5-10.6$ ] } & $2.95 \mathrm{E}-15$ \\
\hline RNPEP & PERIVENOUS & arginyl aminopeptidase (aminopeptidase B) & {$[10.04-10.09-10.14]$} & {$[$ [ $10.39-10.46-10.53]$} & {$[10.05-10.12-10.18]$} & {$[9.9-9.97-10.04$ ] } & $1.74 \mathrm{E}-14$ \\
\hline EIF2B4 & PERIVENOUS & eukaryotic translation initiation factor $2 \mathrm{~B}$, subunit 4 delta, $67 \mathrm{kDa}$ & {$[9.68-9.74-9.8]$} & {$[10.36-10.44-10.53]$} & {$[9.76-9.83-9.91]$} & {$[9.87-9.96-10.05]$} & $2.97 \mathrm{E}-25$ \\
\hline ATRN & PERIVENOUS & attractin & $\mid[10.58-10.64-10.71]$ & {$[10.35-10.44-10.53]$} & {$[10.41-10.49-10.57$ ] } & {$[10-10.11-10.21]$} & 7.96E-92 \\
\hline CYP1A2 & PERIVENOUS & cytochrome P450, family 1, subfamily A, polypeptide 2 & {$[9.17-9.32-9.47]$} & {$[10.19-10.4-10.61]$} & {$[8.6-8.75-8.9]$} & {$[8.02-8.14-8.26]$} & $8.21 \mathrm{E}-16$ \\
\hline
\end{tabular}

Hepatology

This article is protected by copyright. All rights reserved. 


\begin{tabular}{|c|c|c|c|c|c|c|c|}
\hline TIMM17A & PERIVENOUS & translocase of inner mitochondrial membrane 17 homolog A (yeast) & {$[9.64-9.7-9.77$ ] } & {$[10.31-10.4-10.48]$} & {$[9.6-9.66-9.73]$} & {$[9.35-9.43-9.51]$} & $1.03 \mathrm{E}-40$ \\
\hline CYB5B & PERIVENOUS & cytochrome b5 type B (outer mitochondrial membrane) & {$[9.61-9.66-9.71]$} & {$[10.26-10.35-10.43]$} & {$[9.65-9.72-9.78]$} & {$[9.72-9.8-9.88]$} & $1.35 \mathrm{E}-45$ \\
\hline LGTN & PERIVENOUS & ligatin & {$[9.86-9.92-9.97]$} & {$[10.27-10.34-10.42]$} & {$[10.06-10.12-10.18]$} & {$[10.01-10.08-10.16]$} & 7.23E-43 \\
\hline CAP2 & PERIVENOUS & CAP, adenylate cyclase-associated protein, 2 (yeast) & {$[9.4-9.5-9.59]$} & {$[10.23-10.34-10.45]$} & {$[9.54-9.64-9.74$ ] } & {$[9.4-9.52-9.65]$} & $1.34 \mathrm{E}-16$ \\
\hline TGIF1 & PERIVENOUS & TGFB-induced factor homeobox 1 & {$[9.51-9.6-9.69]$} & {$[10.21-10.31-10.41]$} & {$[9.67-9.76-9.84$ ] } & {$[9.72-9.82-9.93]$} & $2.97 \mathrm{E}-33$ \\
\hline ZNF238 & PERIVENOUS & Zinc finger protein 238 & {$[9.51-9.58-9.65]$} & {$[10.19-10.3-10.4]$} & {$[9.59-9.67-9.75]$} & {$[9.25-9.37-9.49]$} & $1.46 \mathrm{E}-63$ \\
\hline HNRNPH2 & PERIVENOUS & heterogeneous nuclear ribonucleoprotein $\mathrm{H} 2\left(\mathrm{H}^{\prime}\right)$ & {$[10.03-10.07-10.11]$} & $|[10.21-10.27-10.33]|$ & {$[9.96-10.01-10.05]$} & [ $9.93-9.98-10.03]$ & 1.74E-20 \\
\hline $\mathrm{PDHX}$ & PERIVENOUS & pyruvate dehydrogenase complex, component $\mathrm{X}$ & {$[9.95-9.99-10.03]$} & {$[10.16-10.23-10.3]$} & {$[9.85-9.9-9.95]$} & {$[9.8-9.86-9.92]$} & 1.16E-51 \\
\hline NEDD4 & PERIVENOUS & neural precursor cell expressed, developmentally down-regulated 4 & {$[9.51-9.59-9.67]$} & {$[10.11-10.21-10.3]$} & {$[9.3-9.39-9.48]$} & {$[8.8-8.92-9.04]$} & $4.91 \mathrm{E}-46$ \\
\hline PFDN6 & PERIVENOUS & prefoldin subunit 6 & {$[9.77-9.82-9.88]$} & {$[10.11-10.19-10.27]$} & {$[9.7-9.76-9.83]$} & {$[9.87-9.95-10.03]$} & $5.65 \mathrm{E}-10$ \\
\hline SMAD3 & PERIVENOUS & SMAD family member 3 & {$[9.4-9.45-9.5]$} & {$[10.07-10.12-10.18]$} & [ 9.55 - $9.61-9.67$ ] & {$[9.74-9.81-9.89]$} & $3.15 \mathrm{E}-38$ \\
\hline HEXA & PERIVENOUS & hexosaminidase A (alpha polypeptide) & {$[9.58-9.63-9.68]$} & {$[10.01-10.09-10.17]$} & {$[9.47-9.53-9.59]$} & {$[9.58-9.66-9.74$ ] } & $2.93 \mathrm{E}-31$ \\
\hline CYP7A1 & PERIVENOUS & cytochrome P450, family 7, subfamily A, polypeptide 1 & {$[10.05-10.24-10.44]$} & {$[9.84-10.07-10.31]$} & {$[9.06-9.27-9.49]$} & {$[7.07-7.31-7.55]$} & 1.46E-24 \\
\hline LGR5 & PERIVENOUS & leucine-rich repeat-containing $\mathrm{G}$ protein-coupled receptor 5 & {$[6.24-6.36-6.49]$} & {$[9.86-10.04-10.22]$} & {$[6.81-6.99-7.16]$} & {$[7.64-7.89-8.13]$} & $2.12 \mathrm{E}-18$ \\
\hline ABLIM1 & PERIVENOUS & actin binding LIM protein 1 & {$[9.36-9.42-9.48]$} & {$[9.95-10.03-10.11]$} & {$[9.31-9.37-9.43]$} & {$[9.5-9.57-9.64]$} & $4.13 \mathrm{E}-54$ \\
\hline TCEAL1 & PERIVENOUS & transcription elongation factor A (SII)-like 1 & {$[9.18-9.24-9.29]$} & {$[9.9-9.99-10.08]$} & {$[9.08-9.15-9.22]$} & {$[8.94-9.03-9.11]$} & $4.18 \mathrm{E}-20$ \\
\hline TNFAIP3 & PERIVENOUS & tumor necrosis factor, alpha-induced protein 3 & {$[9.24-9.31-9.38]$} & {$[9.83-9.95-10.06]$} & {$[9.63-9.7-9.77]$} & {$[9.55-9.65-9.74]$} & $4.77 \mathrm{E}-20$ \\
\hline WIPI2 & PERIVENOUS & WD repeat domain, phosphoinositide interacting 2 & {$[9.65-9.69-9.73]$} & {$[9.88-9.94-10]$} & {$[9.59-9.64-9.69]$} & {$[9.64-9.7-9.75]$} & $5.04 \mathrm{E}-04$ \\
\hline OSER1 & PERIVENOUS & oxidative stress responsive serine-rich 1 & [9.51-9.57-9.63] & {$[9.87-9.93-9.98]$} & [9.42- $9.47-9.53]$ & {$[9.33-9.4-9.46]$} & $1.27 \mathrm{E}-35$ \\
\hline CBFB & PERIVENOUS & core-binding factor, beta subunit & {$[9.65-9.71-9.77]$} & {$[9.84-9.91-9.97$ ] } & {$[9.82-9.88-9.94]$} & {$[$ [ $10.12-10.19-10.27]$} & $3.13 \mathrm{E}-49$ \\
\hline NARS2 & PERIVENOUS & asparaginyl-tRNA synthetase 2, mitochondrial (putative) & {$[9.37-9.42-9.48]$} & {$[9.82-9.88-9.94]$} & {$[9.4-9.46-9.52]$} & {$[9.37-9.44-9.51]$} & $2.84 \mathrm{E}-14$ \\
\hline ICK & PERIVENOUS & intestinal cell (MAK-like) kinase & {$[8.41-8.48-8.55]$} & {$[9.73-9.87-10.01]$} & {$[8.37-8.46-8.55]$} & {$[8.51-8.61-8.7]$} & $1.62 \mathrm{E}-32$ \\
\hline LAMA3 & PERIVENOUS & laminin, alpha 3 & {$[7.91-8-8.09]$} & {$[9.71-9.86-10.02]$} & {$[7.92-8.01-8.11]$} & {$[7.61-7.74-7.87]$} & $9.73 \mathrm{E}-25$ \\
\hline YAP1 & PERIVENOUS & Yes-associated protein 1 & {$[9.11-9.17-9.23]$} & {$[9.77-9.86-9.94]$} & {$[9.25-9.32-9.4]$} & {$[9.47-9.55-9.64]$} & $3.67 \mathrm{E}-22$ \\
\hline DNAJC12 & PERIVENOUS & DnaJ (Hsp40) homolog, subfamily C, member 12 & {$[8.6-8.73-8.86]$} & {$[9.66-9.85-10.05]$} & {$[8.34-8.48-8.63]$} & {$[8.3-8.48-8.67]$} & $4.58 \mathrm{E}-16$ \\
\hline TMCC1 & PERIVENOUS & transmembrane and coiled-coil domain family 1 & {$[9-9.09-9.18]$} & {$[9.72-9.85-9.98]$} & {$[9.12-9.22-9.32]$} & {$[9.25-9.36-9.46]$} & $6.55 \mathrm{E}-27$ \\
\hline ANKRA2 & PERIVENOUS & ankyrin repeat, family A (RFXANK-like), 2 & [9.36- $9.41-9.47]$ & {$[9.76-9.84-9.92]$} & {$[9.24-9.3-9.36]$} & [9.04-9.12-9.19] & $4.93 \mathrm{E}-38$ \\
\hline THEM6 & PERIVENOUS & thioesterase superfamily member 6 & {$[9.43-9.5-9.56]$} & {$[9.73-9.81-9.9]$} & {$[9.42-9.5-9.57]$} & {$[9.14-9.23-9.32$ ] } & $3.81 \mathrm{E}-58$ \\
\hline MTR & PERIVENOUS & 5-methyltetrahydrofolate-homocysteine methyltransferase & {$[9.04-9.1-9.16]$} & {$[9.69-9.78-9.86]$} & {$[9.32-9.39-9.47]$} & {$[9.23-9.31-9.39]$} & $8.16 \mathrm{E}-23$ \\
\hline AIMP1 & PERIVENOUS & aminoacyl tRNA synthetase complex-interacting multifunctional protein 1 & {$[9.21-9.26-9.32$ ] } & {$[9.67-9.75-9.84$ ] } & {$[9.18-9.26-9.33$ ] } & {$[9.31-9.39-9.47$ ] } & $1.17 \mathrm{E}-28$ \\
\hline
\end{tabular}

Hepatology

This article is protected by copyright. All rights reserved. 


\begin{tabular}{|c|c|c|c|c|c|c|c|}
\hline ARHGAP12 & PERIVENOUS & Rho GTPase activating protein 12 & {$[8.84-8.9-8.97]$} & {$[9.64-9.73-9.82]$} & {$[9.13-9.21-9.28]$} & {$[9.16-9.24-9.33]$} & $3.69 \mathrm{E}-22$ \\
\hline THAP11 & PERIVENOUS & THAP domain containing 11 & {$[9.26-9.31-9.35]$} & {$[9.62-9.67-9.72]$} & {$[9.26-9.31-9.36]$} & {$[9.43-9.49-9.55]$} & $1.97 \mathrm{E}-41$ \\
\hline PARN & PERIVENOUS & poly(A)-specific ribonuclease (deadenylation nuclease) & {$[9.35-9.4-9.44]$} & {$[9.61-9.66-9.71]$} & {$[9.27-9.32-9.38]$} & {$[9.15-9.22-9.29]$} & $1.29 \mathrm{E}-24$ \\
\hline ASAP2 & PERIVENOUS & ArfGAP with $\mathrm{SH} 3$ domain, ankyrin repeat and $\mathrm{PH}$ domain 2 & {$[8.56-8.64-8.72]$} & {$[9.53-9.63-9.73]$} & {$[8.84-8.91-8.98]$} & {$[8.99-9.09-9.2]$} & $9.05 \mathrm{E}-03$ \\
\hline GNAl1 & PERIVENOUS & $\begin{array}{l}\text { guanine nucleotide binding protein ( } G \text { protein), alpha inhibiting activity } \\
\text { polypeptide } 1\end{array}$ & {$[8.6-8.67-8.74]$} & [ $9.48-9.6-9.71]$ & {$[8.6-8.69-8.78]$} & {$[8.61-8.7-8.79]$} & $5.70 \mathrm{E}-22$ \\
\hline PPP1R2 & PERIVENOUS & protein phosphatase 1, regulatory (inhibitor) subunit 2 & {$[9.01-9.05-9.1]$} & {$[9.47-9.54-9.61]$} & {$[9.06-9.12-9.17]$} & {$[9.21-9.28-9.35]$} & $1.60 \mathrm{E}-11$ \\
\hline NCOA2 & PERIVENOUS & nuclear receptor coactivator 2 & {$[8.83-8.9-8.96]$} & {$[9.38-9.48-9.58]$} & {$[8.88-8.97-9.05]$} & {$[8.71-8.81-8.9]$} & $4.13 \mathrm{E}-12$ \\
\hline PRKCA & PERIVENOUS & protein kinase C, alpha & {$[8.95-9.01-9.07]$} & {$[9.36-9.44-9.52]$} & {$[8.95-9.02-9.09]$} & {$[8.86-8.95-9.04]$} & $1.63 \mathrm{E}-39$ \\
\hline SOX13 & PERIVENOUS & SRY (sex determining region Y)-box 13 & {$[9.2-9.26-9.31]$} & {$[9.36-9.42-9.49]$} & {$[9.46-9.52-9.58]$} & {$[9.43-9.52-9.61]$} & $4.73 \mathrm{E}-17$ \\
\hline TLE1 & PERIVENOUS & transducin-like enhancer of split 1 (E(sp1) homolog, Drosophila) & {$[8.9-8.95-9]$} & {$[9.34-9.42-9.49]$} & {$[8.95-9.02-9.09]$} & {$[8.93-9.02-9.11]$} & $1.01 \mathrm{E}-27$ \\
\hline MTMR11 & PERIVENOUS & myotubularin related protein 11 & {$[8.41-8.5-8.6]$} & {$[9.29-9.41-9.53]$} & {$[8.65-8.76-8.86]$} & {$[8.51-8.64-8.77]$} & $6.69 \mathrm{E}-09$ \\
\hline SLC30A10 & PERIVENOUS & solute carrier family 30 , member 10 & {$[8.61-8.69-8.76]$} & {$[9.26-9.38-9.5]$} & {$[8.57-8.67-8.77]$} & {$[8.22-8.36-8.51]$} & $2.43 \mathrm{E}-23$ \\
\hline RRNAD1 & PERIVENOUS & ribosomal RNA adenine dimethylase domain containing 1 & {$[9.09-9.14-9.19]$} & {$[9.3-9.37-9.43]$} & {$[8.92-8.98-9.04]$} & {$[8.81-8.88-8.95]$} & $3.97 \mathrm{E}-18$ \\
\hline HEATR1 & PERIVENOUS & HEAT repeat containing 1 & {$[8.66-8.72-8.78]$} & {$[9.24-9.33-9.41]$} & {$[9.14-9.2-9.27]$} & {$[9.34-9.42-9.5]$} & $5.01 \mathrm{E}-16$ \\
\hline TERF2 & PERIVENOUS & telomeric repeat binding factor 2 & {$[8.88-8.92-8.96]$} & {$[9.23-9.28-9.34]$} & {$[8.85-8.9-8.95]$} & {$[8.81-8.86-8.92]$} & $6.92 \mathrm{E}-32$ \\
\hline NBAS & PERIVENOUS & neuroblastoma amplified sequence & {$[8.87-8.91-8.96]$} & {$[9.18-9.25-9.32]$} & {$[8.89-8.93-8.97]$} & {$[8.9-8.95-9]$} & $6.65 \mathrm{E}-17$ \\
\hline PARVB & PERIVENOUS & parvin, beta & {$[8.25-8.32-8.4]$} & {$[9.08-9.22-9.37]$} & {$[8.61-8.7-8.79]$} & {$[8.66-8.76-8.86]$} & $1.40 \mathrm{E}-30$ \\
\hline RCBTB1 & PERIVENOUS & $\begin{array}{l}\text { regulator of chromosome condensation (RCC1) and BTB (POZ) domain } \\
\text { containing protein } 1\end{array}$ & {$[8.51-8.57-8.63]$} & {$[9.13-9.21-9.29]$} & {$[8.5-8.57-8.63]$} & {$[8.63-8.7-8.78]$} & 1.47E-19 \\
\hline PTPRG & PERIVENOUS & protein tyrosine phosphatase, receptor type, $\mathrm{G}$ & {$[8.29-8.35-8.4]$} & {$[9.08-9.16-9.25]$} & {$[8.35-8.42-8.5]$} & {$[8.67-8.75-8.84]$} & $1.31 \mathrm{E}-67$ \\
\hline NAGPA & PERIVENOUS & $\mathrm{N}$-acetylglucosamine-1-phosphodiester alpha-N-acetylglucosaminidase & {$[8.73-8.79-8.85]$} & {$[9.05-9.14-9.22]$} & {$[8.63-8.69-8.76]$} & {$[8.61-8.69-8.76]$} & $9.66 \mathrm{E}-06$ \\
\hline TRAPPC6A & PERIVENOUS & trafficking protein particle complex $6 \mathrm{~A}$ & {$[8.95-9.01-9.08]$} & {$[9.01-9.09-9.17]$} & {$[8.75-8.82-8.89]$} & {$[8.59-8.67-8.76]$} & $2.16 \mathrm{E}-27$ \\
\hline RAB11FIP2 & PERIVENOUS & RAB11 family interacting protein 2 (class I) & {$[8.2-8.26-8.32]$} & {$[8.92-8.99-9.07]$} & {$[8.38-8.45-8.51]$} & {$[8.43-8.5-8.58]$} & $2.15 \mathrm{E}-44$ \\
\hline HS1BP3 & PERIVENOUS & HCLS1 binding protein 3 & {$[8.54-8.6-8.66]$} & {$[8.89-8.97-9.05]$} & {$[8.53-8.59-8.66]$} & {$[8.38-8.45-8.52]$} & $3.44 \mathrm{E}-34$ \\
\hline THADA & PERIVENOUS & thyroid adenoma & {$[8.16-8.22-8.28]$} & {$[8.83-8.92-9]$} & {$[8.41-8.47-8.53]$} & {$[8.6-8.66-8.73]$} & $3.53 \mathrm{E}-26$ \\
\hline AP2B1 & PERIVENOUS & adaptor-related protein complex 2, beta 1 subunit & {$[8.34-8.4-8.46]$} & {$[8.8-8.88-8.96]$} & {$[8.34-8.41-8.47]$} & {$[8.62-8.71-8.79]$} & $1.11 \mathrm{E}-13$ \\
\hline CASK & PERIVENOUS & calcium/calmodulin-dependent serine protein kinase (MAGUK family) & {$[8.39-8.44-8.5]$} & {$[8.8-8.88-8.96]$} & {$[8.45-8.51-8.57]$} & {$[8.49-8.57-8.65]$} & 1.60E-27 \\
\hline NUP93 & PERIVENOUS & nucleoporin 93kDa & {$[8.48-8.54-8.6]$} & {$[8.79-8.87-8.96]$} & {$[8.71-8.78-8.85]$} & {$[9.13-9.22-9.3]$} & $4.22 \mathrm{E}-64$ \\
\hline STAM2 & PERIVENOUS & signal transducing adaptor molecule (SH3 domain and ITAM motif) 2 & {$[8.43-8.49-8.55]$} & {$[8.8-8.87-8.94]$} & {$[8.35-8.41-8.48]$} & {$[8.43-8.5-8.57]$} & $1.21 \mathrm{E}-25$ \\
\hline DIEXF & PERIVENOUS & digestive organ expansion factor homolog (zebrafish) & {$[8.39-8.45-8.51]$} & {$[8.77-8.85-8.93]$} & {$[8.42-8.49-8.57]$} & {$[8.45-8.54-8.62]$} & $1.36 \mathrm{E}-13$ \\
\hline CYP1A1 & PERIVENOUS & cytochrome P450, family 1, subfamily A, polypeptide 1 & {$[6.64-6.77-6.9]$} & {$[8.53-8.77-9.01]$} & {$[6.23-6.36-6.5]$} & {$[5.89-6.02-6.16]$} & $6.72 \mathrm{E}-35$ \\
\hline IMPAD1 & PERIVENOUS & inositol monophosphatase domain contain & {$[8.03-8.09-8.14]$} & {$[8.51-8.58-8.66]$} & {$[7.98-8.04-8.1]$} & {$[8.04-8.11-8.18]$} & $3.61 \mathrm{E}-43$ \\
\hline KATNB1 & PERIVENOUS & katanin p80 (WD repeat containing) subunit B 1 & {$[8.01-8.07-8.12]$} & {$[8.42-8.49-8.56]$} & {$[8.05-8.11-8.17]$} & {$[8.11-8.18-8.25]$} & $8.02 \mathrm{E}-22$ \\
\hline SLC7A6 & PERIVENOUS & $\begin{array}{l}\text { solute carrier family } 7 \text { (cationic amino acid transporter, } y+\text { system), } \\
\text { member } 6\end{array}$ & {$[7.51-7.57-7.62]$} & {$[8.37-8.45-8.53]$} & {$[7.92-7.99-8.05]$} & {$[8.16-8.24-8.32]$} & $5.35 \mathrm{E}-18$ \\
\hline CEP68 & PERIVENOUS & centrosomal protein $68 \mathrm{kDa}$ & {$[7.82-7.89-7.97]$} & {$[8.36-8.44-8.51]$} & {$[7.96-8.03-8.11]$} & {$[8.12-8.2-8.28]$} & $2.55 \mathrm{E}-14$ \\
\hline RAD50 & PERIVENOUS & og (S. cerevisiae) & {$[8.09-8.16-8.22]$} & {$[8.31-8.4-8.5]$} & {$[7.91-7.98-8.04]$} & {$[7.81-7.9-7.98]$} & $2.40 \mathrm{E}-12$ \\
\hline MID1 & PERIVENOUS & midline 1 (Opitz/BBB syndrome) & {$[7.47-7.54-7.6]$} & {$[8.11-8.21-8.3]$} & {$[7.62-7.71-7.8]$} & {$[7.69-7.78-7.88]$} & $4.67 \mathrm{E}-25$ \\
\hline GREB1 & PERIVENOUS & growth regulation by estrogen in breast cancer 1 & {$[6.87-6.95-7.03]$} & {$[7.97-8.11-8.25]$} & {$[6.88-6.98-7.07]$} & {$[7.08-7.22-7.36]$} & $5.19 \mathrm{E}-35$ \\
\hline
\end{tabular}




\begin{tabular}{|c|c|c|c|c|c|c|c|}
\hline LEF1 & PERIVENOUS & lymphoid enhancer-binding factor 1 & {$[6.37-6.45-6.52]$} & {$[7.28-7.42-7.55]$} & {$[6.8-6.89-6.98]$} & {$[6.82-6.92-7.03]$} & $1.29 \mathrm{E}-40$ \\
\hline RPL8 & ECM/STEM & ribosomal protein L8 & {$[12.99-13.05-13.1]$} & [ $13.24-13.33-13.41]$ & [ $13.37-13.43-13.49]$ & {$[13.66-13.72-13.78]$} & $8.23 \mathrm{E}-13$ \\
\hline RPS3 & ECM/STEM & ribosomal protein S3 & [ 12.62 - $12.67-12.72$ ] & [ $12.79-12.86-12.93]$ & [ $12.91-12.97-13.02]$ & [ [ $13.41-13.47-13.52$ ] & $6.53 \mathrm{E}-24$ \\
\hline PABPC1 & ECM/STEM & poly(A) binding protein, cytoplasmic 1 & {$[12.7-12.75-12.8]$} & {$[13.07-13.15-13.22]$} & [ $13.11-13.17-13.22]$ & [ $13.39-13.45-13.51]$ & $3.67 \mathrm{E}-56$ \\
\hline TUBA1C & ECM/STEM & tubulin, alpha 1c & {$[12.34-12.39-12.44$ ] } & {$[12.37-12.44-12.5]$} & {$[12.65-12.69-12.74$ ] } & {$[12.99-13.05-13.1]$} & $7.80 \mathrm{E}-54$ \\
\hline EIF3H & ECM/STEM & eukaryotic translation initiation factor 3 , subunit $\mathrm{H}$ & {$[12.36-12.4-12.45]$} & [ $12.72-12.79-12.86$ ] & {$[12.59-12.65-12.71]$} & {$[12.91-12.97-13.04$ ] } & $1.07 \mathrm{E}-60$ \\
\hline ENO1 & ECM/STEM & enolase 1, (alpha) & {$[12.33-12.38-12.43]$} & [ $12.41-12.49-12.57$ ] & {$[12.37-12.45-12.53]$} & {$[12.87-12.96-13.05]$} & $1.40 \mathrm{E}-54$ \\
\hline HSPA5 & ECM/STEM & heat shock $70 \mathrm{kDa}$ protein 5 (glucose-regulated protein, $78 \mathrm{kDa}$ ) & {$[12.55-12.6-12.65]$} & {$[12.27-12.33-12.39]$} & [ [ $12.66-12.71-12.76]$ & {$[12.67-12.72-12.77$ ] } & $6.14 \mathrm{E}-43$ \\
\hline DYNLL1 & ECM/STEM & dynein, light chain, LC8-type 1 & {$[12.31-12.35-12.38$ ] } & {$[12.37-12.42-12.47]$} & {$[12.52-12.55-12.58]$} & {$[12.64-12.68-12.72$ ] } & $2.57 \mathrm{E}-53$ \\
\hline ARPC2 & ECM/STEM & actin related protein $2 / 3$ complex, subunit $2,34 \mathrm{kDa}$ & {$[11.94-11.97-12.01]$} & {$[11.95-12-12.06]$} & {$[12.23-12.28-12.33]$} & {$[12.5-12.56-12.61]$} & $3.73 E-48$ \\
\hline CFL1 & ECM/STEM & cofilin 1 (non-muscle) & {$[12.15-12.19-12.23$ ] } & {$[12.16-12.21-12.26]$} & {$[12.31-12.35-12.39]$} & [ 12.5 - 12.54 - 12.59] & $2.21 \mathrm{E}-44$ \\
\hline GPI & ECM/STEM & glucose-6-phosphate isomerase & [ $12.26-12.32-12.37$ ] & [ $12.01-12.09-12.16$ ] & [ $12.41-12.46-12.51]$ & [ $12.44-12.51-12.57$ ] & $8.89 \mathrm{E}-41$ \\
\hline YWHAZ & ECM/STEM & $\begin{array}{l}\text { tyrosine 3-monooxygenase/tryptophan 5-monooxygenase activation } \\
\text { protein, zeta polypeptide }\end{array}$ & {$[11.72-11.78-11.83]$} & [ $11.82-11.9-11.98]$ & {$[12.1-12.17-12.23]$} & [ $12.42-12.49-12.56$ ] & $8.79 \mathrm{E}-55$ \\
\hline PPA1 & ECM/STEM & pyrophosphatase (inorganic) 1 & {$[12.08-12.13-12.18]$} & [ $12.21-12.28-12.35]$ & [ $12.37-12.43-12.48]$ & [ $12.42-12.49-12.56$ ] & $3.30 \mathrm{E}-12$ \\
\hline SPP1 & ECM/STEM & secreted phosphoprotein 1 & {$[10.14-10.34-10.55]$} & [ $11.19-11.5-11.81]$ & [ $11.64-11.85-12.06]$ & [ $12.13-12.34-12.55]$ & $8.59 \mathrm{E}-57$ \\
\hline SPINK1 & ECM/STEM & serine peptidase inhibitor, Kazal type 1 & {$[10.68-10.96-11.25]$} & [ $9.32-9.71-10.11]$ & [ 12.45 - 12.68 - 12.91 ] & [ $11.94-12.23-12.51]$ & $5.54 \mathrm{E}-30$ \\
\hline |F|30 & ECM/STEM & interferon, gamma-inducible protein 30 & {$[11.61-11.68-11.75]$} & [ $11.44-11.54-11.65$ ] & [ $11.91-11.99-12.08]$ & [ $12.11-12.22-12.33$ ] & $2.40 \mathrm{E}-60$ \\
\hline CLIC1 & ECM/STEM & chloride intracellular channel 1 & {$[11.17-11.23-11.29]$} & [ $11.4-11.47-11.53$ ] & {$[11.55-11.6-11.65]$} & {$[12.14-12.19-12.25]$} & $7.00 \mathrm{E}-41$ \\
\hline MAT2A & ECM/STEM & methionine adenosyltransferase II, alpha & {$[11.72-11.77-11.82$ ] } & [ $11.48-11.56-11.63]$ & [ $11.92-11.97-12.02$ ] & {$[12.13-12.19-12.26]$} & $3.86 \mathrm{E}-40$ \\
\hline CAP1 & ECM/STEM & CAP, adenylate cyclase-associated protein 1 (yeast) & [ $11.62-11.66-11.7]$ & {$[11.69-11.73-11.77$ ] } & {$[11.71-11.76-11.8]$} & {$[11.93-11.99-12.05]$} & $5.81 \mathrm{E}-28$ \\
\hline SPATS2L & ECM/STEM & spermatogenesis associated, serine-rich 2-like & {$[11.32-11.37-11.43]$} & {$[11.13-11.2-11.27]$} & {$[11.5-11.56-11.62]$} & [ $11.84-11.91-11.99]$ & $2.48 \mathrm{E}-35$ \\
\hline ANXA5 & ECM/STEM & annexin A5 & {$[11-11.07-11.15]$} & [ $10.67-10.8-10.93]$ & [ $11.35-11.45-11.55]$ & [ $11.83-11.91-11.99]$ & $1.30 \mathrm{E}-46$ \\
\hline
\end{tabular}

Hepatology

This article is protected by copyright. All rights reserved. 


\begin{tabular}{|c|c|c|c|c|c|c|c|}
\hline EIF3E & ECM/STEM & eukaryotic translation initiation factor 3 , subunit $\mathrm{E}$ & {$[11.25-11.31-11.37]$} & {$[11.86-11.95-12.04$ ] } & {$[11.58-11.64-11.7]$} & [ $11.82-11.9-11.99]$ & $2.96 \mathrm{E}-86$ \\
\hline NDRG1 & ECM/STEM & $\mathrm{N}$-myc downstream regulated 1 & {$[10.73-10.82-10.91]$} & [ $10.96-11.07-11.17$ ] & {$[11.33-11.43-11.53]$} & [ $11.78-11.9-12.02]$ & $5.17 \mathrm{E}-22$ \\
\hline BCAP31 & ECM/STEM & B-cell receptor-associated protein 31 & {$[11.45-11.5-11.54]$} & {$[11.95-12.02-12.08]$} & {$[11.67-11.72-11.78]$} & {$[11.77-11.83-11.88]$} & $2.41 \mathrm{E}-33$ \\
\hline ATIC & ECM/STEM & $\begin{array}{l}\text { 5-aminoimidazole-4-carboxamide ribonucleotide formyltransferase/IMP } \\
\text { cyclohydrolase }\end{array}$ & {$[11.01-11.06-11.1]$} & {$[11.28-11.34-11.41]$} & {$[11.28-11.33-11.39]$} & {$[11.69-11.76-11.82]$} & $1.09 \mathrm{E}-30$ \\
\hline H2AFZ & ECM/STEM & H2A histone family, member Z & {$[11.03-11.11-11.18]$} & {$[11.05-11.13-11.22]$} & {$[11.18-11.27-11.35]$} & {$[11.67-11.75-11.83]$} & $6.60 \mathrm{E}-32$ \\
\hline TM4SF1 & ECM/STEM & transmembrane $4 \mathrm{~L}$ six family member 1 & {$[10.69-10.78-10.86]$} & {$[10.88-11-11.13]$} & {$[11-11.11-11.22]$} & {$[11.59-11.73-11.87]$} & $2.10 \mathrm{E}-44$ \\
\hline CBX3 & ECM/STEM & chromobox homolog 3 & {$[11.06-11.11-11.15]$} & {$[11.28-11.34-11.4]$} & {$[11.26-11.31-11.36]$} & {$[11.63-11.69-11.75]$} & $5.85 \mathrm{E}-36$ \\
\hline TFRC & ECM/STEM & transferrin receptor $(\mathrm{p} 90, \mathrm{CD} 71)$ & {$[10.93-11-11.08]$} & {$[10.61-10.72-10.82]$} & {$[11.26-11.34-11.42]$} & {$[11.59-11.68-11.77]$} & 1.17E-24 \\
\hline BAMBI & ECM/STEM & BMP and activin membrane-bound inhibitor homolog (Xenopus laevis) & {$[10.04-10.18-10.32]$} & {$[11.35-11.48-11.61]$} & {$[10.81-10.92-11.03]$} & {$[11.54-11.65-11.77]$} & $1.00 \mathrm{E}-40$ \\
\hline KPNB1 & ECM/STEM & karyopherin (importin) beta 1 & {$[11.05-11.1-11.15]$} & {$[11.21-11.27-11.33]$} & {$[11.21-11.26-11.31]$} & {$[11.57-11.63-11.69]$} & $9.91 \mathrm{E}-52$ \\
\hline WDR1 & ECM/STEM & WD repeat domain 1 & {$[11.1-11.14-11.17]$} & {$[11.28-11.33-11.39]$} & {$[11.36-11.41-11.46]$} & {$[11.55-11.61-11.67]$} & $1.36 \mathrm{E}-66$ \\
\hline GRN & ECM/STEM & granulin & {$[11.32-11.37-11.42]$} & {$[11.21-11.27-11.34$ ] } & {$[11.34-11.4-11.46]$} & {$[11.51-11.59-11.68]$} & $8.10 \mathrm{E}-32$ \\
\hline HIF1A & ECM/STEM & $\begin{array}{l}\text { hypoxia inducible factor 1, alpha subunit (basic helix-loop-helix } \\
\text { transcription factor) }\end{array}$ & {$[10.86-10.91-10.96]$} & {$[10.73-10.8-10.86]$} & {$[11.2-11.26-11.31]$} & {$[11.51-11.59-11.66]$} & $8.89 \mathrm{E}-32$ \\
\hline ACTR3 & ECM/STEM & ARP3 actin-related protein 3 homolog (yeast) & {$[11.13-11.17-11.2]$} & {$[11.16-11.21-11.26]$} & {$[11.3-11.34-11.38]$} & {$[11.54-11.58-11.63]$} & $1.11 \mathrm{E}-41$ \\
\hline ALDOA & ECM/STEM & aldolase A, fructose-bisphosphate & {$[10.81-10.87-10.94]$} & [ $10.85-10.94-11.03$ ] & {$[11.01-11.09-11.18]$} & {$[11.44-11.55-11.67]$} & $6.64 \mathrm{E}-25$ \\
\hline MSN & ECM/STEM & moesin & {$[11.06-11.12-11.19]$} & [ $11.14-11.24-11.34$ ] & {$[11.3-11.39-11.48]$} & {$[11.45-11.55-11.65]$} & $1.32 \mathrm{E}-41$ \\
\hline CLNS1A & ECM/STEM & chloride channel, nucleotide-sensitive, $1 \mathrm{~A}$ & {$[11.14-11.18-11.23]$} & {$[11.41-11.46-11.52$ ] } & {$[11.3-11.35-11.4]$} & {$[11.47-11.54-11.6]$} & $3.43 \mathrm{E}-53$ \\
\hline DHRS7 & ECM/STEM & dehydrogenase/reductase (SDR family) member 7 & {$[11.33-11.4-11.47]$} & [ $10.93-11.04-11.16$ ] & {$[11.54-11.62-11.71]$} & [ $11.4-11.48-11.57]$ & $6.16 \mathrm{E}-28$ \\
\hline$S C D$ & ECM/STEM & stearoyl-CoA desaturase (delta-9-desaturase) & {$[10.58-10.71-10.84]$} & {$[11.5-11.64-11.78]$} & {$[11.4-11.51-11.62]$} & {$[11.33-11.46-11.58]$} & $1.16 \mathrm{E}-50$ \\
\hline NREP & ECM/STEM & neuronal regeneration related protein & {$[10.48-10.57-10.65]$} & [ $10.2-10.32-10.44]$ & {$[10.98-11.07-11.16]$} & {$[11.33-11.45-11.57]$} & $1.18 \mathrm{E}-49$ \\
\hline LGALS3 & ECM/STEM & lectin, galactoside-binding, soluble, 3 & {$[10.78-10.89-11]$} & [ $10.11-10.24-10.37$ ] & {$[11.18-11.29-11.41]$} & {$[11.31-11.45-11.58]$} & $1.78 \mathrm{E}-48$ \\
\hline
\end{tabular}

Hepatology

This article is protected by copyright. All rights reserved. 


\begin{tabular}{|c|c|c|c|c|c|c|c|}
\hline ACSL4 & ECM/STEM & acyl-CoA synthetase long-chain family member 4 & {$[9.93-10.09-10.25]$} & {$[9.8-9.99-10.19]$} & {$[11.29-11.43-11.56]$} & {$[11.3-11.44-11.59]$} & 1.07E-29 \\
\hline MARCKSL1 & ECM/STEM & MARCKS-like 1 & {$[10.14-10.21-10.27]$} & {$[10.26-10.37-10.48]$} & {$[10.62-10.69-10.76]$} & {$[11.34-11.44-11.54]$} & $3.03 E-18$ \\
\hline IARS & ECM/STEM & isoleucyl-tRNA synthetase & {$[10.62-10.66-10.71]$} & {$[10.86-10.92-10.99]$} & {$[10.92-10.98-11.03]$} & {$[11.34-11.39-11.45]$} & $1.53 \mathrm{E}-34$ \\
\hline MARCKS & ECM/STEM & myristoylated alanine-rich protein kinase $\mathrm{C}$ substrate & {$[10.05-10.13-10.2]$} & {$[9.94-10.05-10.17$ ] } & {$[10.6-10.69-10.77]$} & {$[11.31-11.39-11.46]$} & $5.66 \mathrm{E}-07$ \\
\hline CALU & ECM/STEM & calumenin & {$[10.88-10.92-10.96$ ] } & {$[10.81-10.87-10.93]$} & {$[11.02-11.07-11.11]$} & {$[11.32-11.37-11.43]$} & 4.27E-31 \\
\hline SPON2 & ECM/STEM & spondin 2, extracellular matrix protein & {$[10.58-10.68-10.77]$} & {$[11.27-11.38-11.48]$} & {$[11.22-11.32-11.42]$} & {$[11.23-11.34-11.46]$} & 3.47E-56 \\
\hline BZW2 & ECM/STEM & basic leucine zipper and W2 domains 2 & [ $10.49-10.53-10.57$ ] & {$[10.81-10.87-10.94]$} & {$[10.7-10.76-10.82]$} & {$[11.27-11.34-11.41]$} & $6.49 \mathrm{E}-60$ \\
\hline HIST1H4C & ECM/STEM & histone cluster $1, \mathrm{H} 4 \mathrm{c}$ & {$[10.71-10.78-10.85]$} & {$[10.35-10.44-10.54]$} & {$[10.77-10.85-10.93]$} & {$[11.25-11.34-11.43]$} & 1.57E-48 \\
\hline DYNC112 & ECM/STEM & dynein, cytoplasmic 1 , intermediate chain 2 & {$[10.81-10.85-10.89]$} & {$[11.04-11.09-11.14]$} & {$[11.01-11.06-11.1]$} & {$[11.28-11.34-11.39]$} & 2.16E-22 \\
\hline CDK4 & ECM/STEM & cyclin-dependent kinase 4 & [ $10.55-10.59-10.64$ ] & {$[10.6-10.67-10.74]$} & {$[10.78-10.83-10.89]$} & {$[11.26-11.32-11.39]$} & 3.05E-61 \\
\hline EPRS & ECM/STEM & glutamyl-prolyl-tRNA synthetase & {$[10.64-10.7-10.75]$} & {$[10.94-11.02-11.09]$} & {$[11.07-11.12-11.18]$} & {$[11.24-11.31-11.38]$} & 3.30E-61 \\
\hline HDAC2 & ECM/STEM & histone deacetylase 2 & {$[10.52-10.56-10.6]$} & {$[10.66-10.73-10.8]$} & {$[10.74-10.8-10.86]$} & {$[11.22-11.28-11.35]$} & $3.02 E-42$ \\
\hline PPT1 & ECM/STEM & palmitoyl-protein thioesterase 1 & {$[10.63-10.69-10.74$ ] } & {$[10.54-10.62-10.71]$} & {$[10.78-10.85-10.93]$} & {$[11.16-11.24-11.32]$} & 7.27E-37 \\
\hline SERPINE2 & ECM/STEM & $\begin{array}{l}\text { serpin peptidase inhibitor, clade } \mathrm{E} \text { (nexin, plasminogen activator inhibitor } \\
\text { type 1), member } 2\end{array}$ & {$[9.95-10.08-10.2]$} & {$[9.59-9.82-10.04]$} & {$[10.71-10.85-10.98]$} & {$[11.09-11.24-11.39]$} & $2.45 \mathrm{E}-66$ \\
\hline TNFRSF21 & ECM/STEM & tumor necrosis factor receptor superfamily, member 21 & {$[9.84-9.92-10]$} & {$[9.72-9.9-10.08]$} & {$[10.38-10.47-10.56]$} & {$[11.14-11.24-11.34]$} & $4.31 \mathrm{E}-18$ \\
\hline COL1A2 & ECM/STEM & collagen, type I, alpha 2 & {$[10.43-10.54-10.65]$} & {$[9.61-9.8-9.99]$} & {$[10.83-10.98-11.13]$} & {$[11.04-11.21-11.38]$} & 3.13E-49 \\
\hline SH3BGRL3 & ECM/STEM & $\mathrm{SH} 3$ domain binding glutamic acid-rich protein like 3 & {$[10.7-10.76-10.82]$} & {$[10.53-10.61-10.69]$} & {$[10.88-10.95-11.02]$} & {$[11.09-11.19-11.29]$} & $6.94 \mathrm{E}-40$ \\
\hline ZMIZ1 & ECM/STEM & zinc finger, MIZ-type containing 1 & {$[10.53-10.58-10.62]$} & {$[10.4-10.46-10.52]$} & {$[10.85-10.91-10.96]$} & {$[11.05-11.12-11.19]$} & 3.14E-57 \\
\hline TSPAN3 & ECM/STEM & tetraspanin 3 & {$[10.65-10.71-10.78]$} & {$[10.08-10.16-10.25]$} & {$[10.75-10.82-10.89]$} & {$[11.02-11.1-11.18]$} & $1.91 \mathrm{E}-57$ \\
\hline SSB & ECM/STEM & Sjogren syndrome antigen B (autoantigen La) & {$[10.41-10.46-10.5]$} & {$[10.69-10.75-10.81]$} & {$[10.68-10.73-10.77]$} & {$[11-11.06-11.13]$} & 5.57E-29 \\
\hline TUBA4A & ECM/STEM & tubulin, alpha $4 a$ & {$[10.49-10.57-10.65]$} & {$[10.22-10.32-10.42]$} & {$[10.85-10.93-11.01]$} & {$[10.93-11.03-11.13]$} & $3.47 \mathrm{E}-35$ \\
\hline
\end{tabular}

Hepatology

This article is protected by copyright. All rights reserved. 


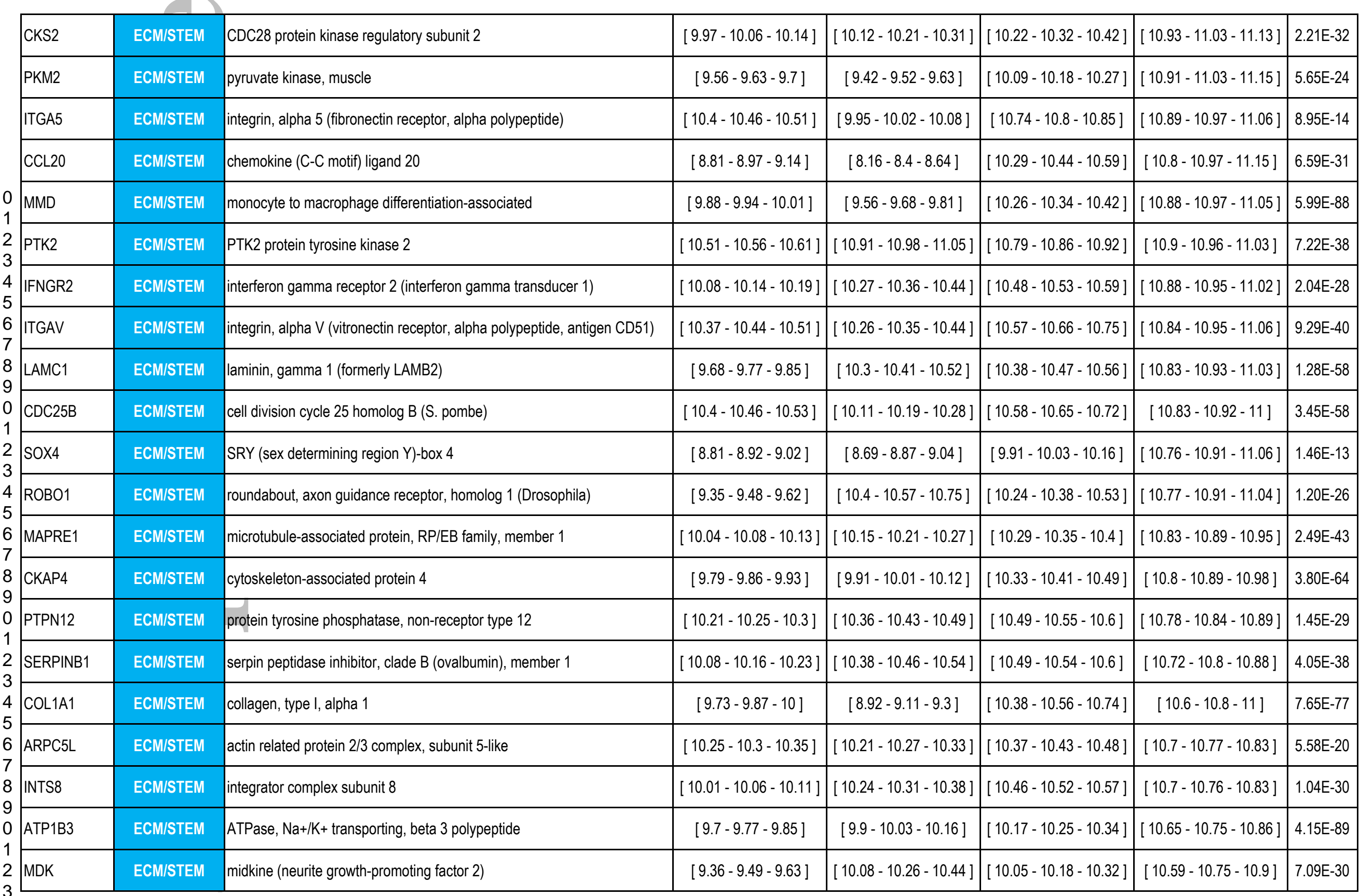

Hepatology

This article is protected by copyright. All rights reserved. 


\begin{tabular}{|c|c|c|c|c|c|c|c|}
\hline CDC42EP4 & ECM/STEM & CDC42 effector protein (Rho GTPase binding) 4 & {$[10.57-10.62-10.67]$} & {$[10.86-10.93-11.01]$} & {$[10.47-10.53-10.59]$} & {$[10.62-10.71-10.79]$} & $3.59 \mathrm{E}-47$ \\
\hline UBE2I & ECM/STEM & ubiquitin-conjugating enzyme E2I (UBC9 homolog, yeast) & {$[10.11-10.17-10.22]$} & {$[10.2-10.27-10.34]$} & {$[10.23-10.3-10.37]$} & {$[10.61-10.69-10.77]$} & $3.10 \mathrm{E}-23$ \\
\hline PVRL2 & ECM/STEM & poliovirus receptor-related 2 (herpesvirus entry mediator B) & {$[10.56-10.62-10.68]$} & {$[10.14-10.21-10.28]$} & {$[10.68-10.75-10.82]$} & {$[10.6-10.68-10.77]$} & 7.84E-11 \\
\hline AMD1 & ECM/STEM & adenosylmethionine decarboxylase 1 & {$[9.96-10.01-10.06]$} & {$[9.99-10.06-10.13]$} & {$[10.29-10.35-10.4]$} & {$[10.61-10.68-10.75]$} & 4.77E-21 \\
\hline MID1IP1 & ECM/STEM & MID1 interacting protein 1 (gastrulation specific G12 homolog (zebrafish)) & {$[9.71-9.79-9.88]$} & {$[10.18-10.27-10.36]$} & {$[10.41-10.48-10.55]$} & {$[10.59-10.68-10.77]$} & 3.67E-43 \\
\hline TMED3 & ECM/STEM & transmembrane emp24 protein transport domain containing 3 & {$[9.26-9.37-9.48]$} & {$[8.78-8.95-9.11]$} & {$[10.03-10.14-10.26]$} & {$[10.53-10.65-10.78]$} & $5.02 \mathrm{E}-48$ \\
\hline S100A11 & ECM/STEM & S100 calcium binding protein A11 & {$[9.63-9.7-9.78]$} & {$[9.52-9.64-9.77]$} & {$[10.08-10.19-10.29]$} & {$[10.5-10.64-10.78]$} & $5.38 \mathrm{E}-72$ \\
\hline TOP2A & ECM/STEM & topoisomerase (DNA) II alpha $170 \mathrm{kDa}$ & {$[8.96-9.1-9.25]$} & {$[9.14-9.3-9.46]$} & {$[9.64-9.76-9.88]$} & {$[10.46-10.59-10.73]$} & $9.81 \mathrm{E}-52$ \\
\hline CAPZA1 & ECM/STEM & capping protein (actin filament) muscle Z-line, alpha 1 & {$[10.04-10.11-10.18]$} & {$[10.07-10.17-10.28]$} & {$[10.15-10.23-10.31]$} & {$[10.49-10.58-10.68]$} & 4.00E-23 \\
\hline IGF2BP2 & ECM/STEM & insulin-like growth factor 2 mRNA binding protein 2 & {$[8.91-9.03-9.16]$} & {$[8.39-8.58-8.76]$} & {$[10.06-10.17-10.29]$} & {$[10.43-10.57-10.72]$} & $1.54 \mathrm{E}-31$ \\
\hline GPNMB & ECM/STEM & glycoprotein (transmembrane) nmb & {$[9.77-9.87-9.97]$} & {$[9.52-9.65-9.78]$} & {$[10-10.14-10.27]$} & {$[10.43-10.57-10.71]$} & $5.78 \mathrm{E}-33$ \\
\hline XPNPEP1 & ECM/STEM & X-prolyl aminopeptidase (aminopeptidase P) 1, soluble & {$[10.12-10.16-10.2]$} & {$[10.28-10.34-10.39]$} & {$[10.39-10.43-10.48]$} & {$[10.51-10.56-10.62]$} & $1.35 \mathrm{E}-59$ \\
\hline ELF3 & ECM/STEM & E74-like factor 3 (ets domain transcription factor, epithelial-specific ) & {$[9.86-9.95-10.03]$} & {$[9.43-9.54-9.65]$} & {$[10.35-10.45-10.54]$} & {$[10.42-10.54-10.66]$} & 5.69E-25 \\
\hline S100P & ECM/STEM & S100 calcium binding protein $\mathrm{P}$ & {$[8.75-8.99-9.24]$} & {$[8.07-8.32-8.56]$} & {$[10.15-10.41-10.68]$} & {$[10.25-10.54-10.84]$} & $9.63 \mathrm{E}-76$ \\
\hline ADM & ECM/STEM & adrenomedullin & {$[9.88-9.97-10.07$ ] } & {$[10.33-10.45-10.57]$} & {$[9.93-10.05-10.17$ ] } & {$[10.38-10.53-10.68]$} & $1.61 \mathrm{E}-62$ \\
\hline SLC38A1 & ECM/STEM & solute carrier family 38, member 1 & {$[9.1-9.24-9.37$ ] } & {$[8.83-9-9.18]$} & {$[9.77-9.91-10.05]$} & {$[10.37-10.53-10.69]$} & $8.70 \mathrm{E}-88$ \\
\hline S100A6 & ECM/STEM & S100 calcium binding protein A6 & {$[9.22-9.32-9.41]$} & {$[8.89-9.02-9.14]$} & {$[9.97-10.1-10.23]$} & {$[10.35-10.52-10.69]$} & $1.71 \mathrm{E}-43$ \\
\hline $\mathrm{RHOQ}$ & ECM/STEM & ras homolog gene family, member $Q$ & {$[10.1-10.15-10.21]$} & {$[9.99-10.07-10.15]$} & {$[10.32-10.39-10.46]$} & {$[10.43-10.51-10.6]$} & 2.03E-38 \\
\hline UBE2Z & ECM/STEM & ubiquitin-conjugating enzyme E2Z & {$[9.81-9.86-9.91]$} & {$[9.96-10.02-10.09]$} & {$[10.01-10.07-10.12]$} & {$[10.39-10.47-10.54]$} & 2.34E-33 \\
\hline VNN1 & ECM/STEM & vanin 1 & {$[11.01-11.15-11.3]$} & [ $8.34-8.58-8.82$ ] & {$[11.1-11.25-11.4]$} & {$[10.24-10.46-10.69]$} & $3.46 \mathrm{E}-45$ \\
\hline SERPINH1 & ECM/STEM & $\begin{array}{l}\text { serpin peptidase inhibitor, clade } \mathrm{H} \text { (heat shock protein } 47 \text { ), member } 1 \text {, } \\
\text { (collagen binding protein 1) }\end{array}$ & [ $9.58-9.64-9.7$ ] & [ $9.25-9.33-9.41]$ & [ $9.82-9.9-9.98$ ] & {$[10.36-10.46-10.56]$} & $3.52 E-44$ \\
\hline
\end{tabular}

Hepatology

This article is protected by copyright. All rights reserved. 


\begin{tabular}{|c|c|c|c|c|c|c|c|}
\hline GNPDA1 & ECM/STEM & glucosamine-6-phosphate deaminase 1 & {$[9.74-9.79-9.85]$} & [ $9.87-9.95-10.03]$ & {$[10.11-10.18-10.25]$} & {$[10.37-10.45-10.52]$} & $1.97 \mathrm{E}-43$ \\
\hline DNMT1 & ECM/STEM & DNA (cytosine-5-)-methyltransferase 1 & {$[9.54-9.61-9.68]$} & {$[9.3-9.38-9.47]$} & [ $9.86-9.94-10.01]$ & {$[10.28-10.36-10.44]$} & $1.79 \mathrm{E}-47$ \\
\hline PELI1 & ECM/STEM & pellino homolog 1 (Drosophila) & {$[9.58-9.65-9.71]$} & {$[9.07-9.15-9.23]$} & [ $9.88-9.94-10.01]$ & {$[10.27-10.34-10.42]$} & 4.14E-30 \\
\hline UBE2C & ECM/STEM & ubiquitin-conjugating enzyme E2C & {$[9.13-9.24-9.35]$} & {$[9.02-9.16-9.3]$} & {$[9.58-9.68-9.78]$} & {$[10.21-10.34-10.46]$} & $1.08 \mathrm{E}-34$ \\
\hline DAB2 & ECM/STEM & disabled homolog 2, mitogen-responsive phosphoprotein (Drosophila) & {$[9.54-9.62-9.7]$} & {$[9.08-9.21-9.33]$} & {$[9.8-9.9-10]$} & {$[10.2-10.31-10.43]$} & $1.29 \mathrm{E}-57$ \\
\hline VPS37C & ECM/STEM & vacuolar protein sorting 37 homolog C (S. cerevisiae) & {$[9.68-9.72-9.76]$} & {$[9.65-9.71-9.78]$} & {$[9.97-10.02-10.07]$} & {$[10.23-10.29-10.35]$} & $1.75 \mathrm{E}-28$ \\
\hline CAMSAP2 & ECM/STEM & calmodulin regulated spectrin-associated protein family, member 2 & {$[9.53-9.59-9.65]$} & {$[9.99-10.07-10.15]$} & {$[10.02-10.08-10.15]$} & {$[10.2-10.27-10.35]$} & $4.59 \mathrm{E}-23$ \\
\hline GLS & ECM/STEM & glutaminase & {$[9.49-9.57-9.66]$} & {$[8.93-9.07-9.21]$} & {$[10.1-10.19-10.28]$} & {$[10.12-10.22-10.33]$} & $8.30 \mathrm{E}-29$ \\
\hline KIAA0196 & ECM/STEM & KIAA0196 & {$[9.74-9.8-9.86]$} & {$[10.15-10.23-10.32$ ] } & {$[10.09-10.16-10.24]$} & {$[10.11-10.19-10.27]$} & 2.26E-21 \\
\hline CHD7 & ECM/STEM & chromodomain helicase DNA binding protein 7 & {$[9.31-9.36-9.42]$} & {$[9.79-9.87-9.95]$} & {$[9.57-9.64-9.72$ ] } & {$[10.09-10.19-10.29]$} & $1.54 \mathrm{E}-37$ \\
\hline PNMA1 & ECM/STEM & paraneoplastic antigen MA1 & {$[8.95-9.03-9.1]$} & {$[8.92-9.02-9.12]$} & [ 9.55 - 9.62 - 9.7$]$ & {$[10.09-10.18-10.27]$} & $1.34 \mathrm{E}-32$ \\
\hline CTSC & ECM/STEM & cathepsin C & {$[9.11-9.2-9.29]$} & {$[9.14-9.28-9.41]$} & {$[9.4-9.49-9.58]$} & {$[10.05-10.17-10.28]$} & $7.29 \mathrm{E}-15$ \\
\hline LGALS4 & ECM/STEM & lectin, galactoside-binding, soluble, 4 & {$[11.39-11.56-11.72]$} & {$[9.43-9.71-9.98]$} & {$[11.25-11.46-11.68]$} & {$[9.86-10.16-10.45]$} & $2.00 \mathrm{E}-34$ \\
\hline CORO1C & ECM/STEM & coronin, actin binding protein, $1 \mathrm{C}$ & {$[9.4-9.45-9.5]$} & {$[9.54-9.63-9.71]$} & {$[9.64-9.7-9.77]$} & {$[10.08-10.15-10.22]$} & $5.65 \mathrm{E}-54$ \\
\hline MMP9 & ECM/STEM & $\begin{array}{l}\text { matrix metallopeptidase } 9 \text { (gelatinase B, 92kDa gelatinase, 92kDa type IV } \\
\text { collagenase) }\end{array}$ & {$[8.19-8.32-8.44]$} & {$[8.17-8.32-8.48]$} & {$[9.2-9.36-9.52]$} & {$[9.94-10.13-10.31]$} & $7.23 \mathrm{E}-43$ \\
\hline HN1 & ECM/STEM & hematological and neurological expressed 1 & {$[9.27-9.33-9.39]$} & {$[9.31-9.4-9.48]$} & {$[9.47-9.53-9.6]$} & {$[10.04-10.12-10.2]$} & $1.65 \mathrm{E}-32$ \\
\hline SUCO & ECM/STEM & SUN domain containing ossification factor & {$[9.43-9.51-9.58]$} & {$[9.83-9.92-10.01]$} & {$[9.86-9.94-10.01]$} & {$[10.02-10.1-10.17]$} & $3.54 \mathrm{E}-34$ \\
\hline LRRC1 & ECM/STEM & leucine rich repeat containing 1 & {$[8.53-8.66-8.79]$} & {$[8.11-8.3-8.49]$} & {$[9.52-9.62-9.72]$} & {$[9.96-10.08-10.21]$} & $2.13 \mathrm{E}-53$ \\
\hline COL5A2 & ECM/STEM & collagen, type $V$, alpha 2 & {$[9.11-9.22-9.32]$} & {$[8.75-8.87-8.99]$} & {$[9.51-9.63-9.75]$} & {$[9.91-10.06-10.22]$} & $1.52 \mathrm{E}-35$ \\
\hline VCAN & ECM/STEM & versican & {$[8.51-8.65-8.79]$} & {$[7.77-7.96-8.15]$} & {$[9.3-9.49-9.68]$} & {$[9.83-10.04-10.24]$} & $7.34 \mathrm{E}-42$ \\
\hline SOX9 & ECM/STEM & SRY (sex determining region Y)-box 9 & {$[8.32-8.46-8.6]$} & {$[8.51-8.72-8.93]$} & {$[9.37-9.5-9.64]$} & {$[9.84-10.04-10.23]$} & $4.08 \mathrm{E}-38$ \\
\hline DRAM1 & ECM/STEM & DNA-damage regulated autophagy modulator 1 & {$[9.25-9.31-9.37]$} & {$[9.34-9.41-9.49]$} & {$[9.56-9.62-9.68]$} & {$[9.95-10.04-10.12]$} & $5.33 \mathrm{E}-37$ \\
\hline PLBD1 & ECM/STEM & phospholipase B domain containing 1 & {$[8.78-8.87-8.96]$} & {$[8.2-8.33-8.45]$} & {$[9.09-9.21-9.32]$} & {$[9.87-10.03-10.18]$} & $2.70 \mathrm{E}-44$ \\
\hline TPX2 & ECM/STEM & TPX2, microtubule-associated, homolog (Xenopus laevis) & {$[8.94-9.03-9.12]$} & {$[8.92-9.04-9.15]$} & {$[9.18-9.27-9.37]$} & {$[9.91-10.02-10.13]$} & $3.64 \mathrm{E}-59$ \\
\hline TMEM165 & ECM/STEM & transmembrane protein 165 & {$[9.4-9.44-9.49]$} & [9.41- $9.48-9.55]$ & {$[9.65-9.7-9.75]$} & {$[9.94-10.01-10.08]$} & $6.13 \mathrm{E}-52$ \\
\hline PAFAH1B3 & ECM/STEM & platelet-activating factor acetylhydrolase $1 \mathrm{~b}$, catalytic subunit $3(29 \mathrm{kDa})$ & {$[8.89-8.97-9.05]$} & {$[8.8-8.9-9]$} & {$[9.41-9.48-9.56$ ] } & [ $9.91-10-10.09]$ & $1.15 \mathrm{E}-51$ \\
\hline
\end{tabular}

Hepatology

This article is protected by copyright. All rights reserved. 


\begin{tabular}{|c|c|c|c|c|c|c|c|}
\hline CTNNAL1 & ECM/STEM & catenin (cadherin-associated protein), alpha-like 1 & {$[9.43-9.5-9.56]$} & {$[10.18-10.26-10.34]$} & {$[9.59-9.67-9.76]$} & {$[9.91-10-10.09]$} & $3.11 \mathrm{E}-12$ \\
\hline UBR5 & ECM/STEM & ubiquitin protein ligase E3 component n-recognin 5 & {$[9.25-9.31-9.37]$} & {$[9.62-9.71-9.79]$} & {$[9.62-9.69-9.76]$} & {$[9.87-9.94-10.01]$} & $4.67 \mathrm{E}-18$ \\
\hline ZNF189 & ECM/STEM & zinc finger protein 189 & {$[9.19-9.26-9.32]$} & {$[9.9-9.98-10.06]$} & {$[9.58-9.66-9.73]$} & {$[9.84-9.92-10.01]$} & $2.15 \mathrm{E}-77$ \\
\hline IL8 & ECM/STEM & interleukin 8 & {$[8.28-8.45-8.62]$} & {$[7.69-7.86-8.03]$} & {$[9.24-9.43-9.63]$} & {$[9.67-9.9-10.12]$} & $3.32 \mathrm{E}-49$ \\
\hline WARS & ECM/STEM & tryptophanyl-tRNA synthetase & [9.33 - $9.39-9.45]$ & {$[9.41-9.49-9.56]$} & {$[9.57-9.64-9.71]$} & [9.78 - $9.89-9.99]$ & $9.56 \mathrm{E}-76$ \\
\hline VIL1 & ECM/STEM & villin 1 & {$[8.25-8.42-8.59]$} & {$[7.76-7.98-8.2]$} & {$[9.24-9.42-9.6]$} & {$[9.66-9.88-10.11]$} & $8.27 \mathrm{E}-36$ \\
\hline SPAG5 & ECM/STEM & sperm associated antigen 5 & {$[9.51-9.58-9.66]$} & {$[8.96-9.07-9.18]$} & {$[9.45-9.54-9.62]$} & {$[9.78-9.88-9.98]$} & $5.44 \mathrm{E}-43$ \\
\hline VAT1 & ECM/STEM & vesicle amine transport protein 1 homolog (T. californica) & {$[9.26-9.32-9.38]$} & {$[9.55-9.65-9.75]$} & {$[9.58-9.65-9.72]$} & {$[9.78-9.86-9.94]$} & $1.20 \mathrm{E}-42$ \\
\hline ETV5 & ECM/STEM & ets variant 5 & {$[8.88-8.95-9.02]$} & {$[8.69-8.79-8.88]$} & [ $9.27-9.36-9.45]$ & {$[9.74-9.84-9.94]$} & $1.72 \mathrm{E}-34$ \\
\hline AGO2 & ECM/STEM & argonaute RISC catalytic component 2 & {$[9-9.06-9.12]$} & {$[9.34-9.43-9.51]$} & {$[9.45-9.52-9.58]$} & {$[9.73-9.81-9.9]$} & $6.42 \mathrm{E}-52$ \\
\hline LHFPL2 & ECM/STEM & lipoma HMGIC fusion partner-like 2 & {$[8.62-8.7-8.78]$} & {$[8.76-8.89-9.02]$} & {$[9.23-9.33-9.43]$} & {$[9.67-9.79-9.91]$} & $6.40 \mathrm{E}-31$ \\
\hline LAMB1 & ECM/STEM & laminin, beta 1 & {$[8.65-8.73-8.81]$} & {$[8.24-8.35-8.46]$} & {$[9.08-9.19-9.3]$} & {$[9.65-9.78-9.9]$} & $1.58 \mathrm{E}-25$ \\
\hline NCAPG & ECM/STEM & non-SMC condensin I complex, subunit G & {$[8.39-8.5-8.61]$} & {$[8.6-8.72-8.84]$} & {$[8.87-8.97-9.08]$} & {$[9.61-9.72-9.83]$} & $1.65 \mathrm{E}-47$ \\
\hline LPCAT1 & ECM/STEM & Iysophosphatidylcholine acyltransferase 1 & {$[8.55-8.62-8.7]$} & {$[8.65-8.77-8.89]$} & {$[9.08-9.17-9.25]$} & {$[9.61-9.7-9.8]$} & $1.49 \mathrm{E}-41$ \\
\hline ARID5B & ECM/STEM & AT rich interactive domain 5B (MRF1-like) & {$[8.84-8.9-8.97]$} & {$[8.79-8.87-8.96]$} & {$[9.25-9.31-9.38]$} & {$[9.6-9.69-9.78]$} & $6.84 \mathrm{E}-21$ \\
\hline YEATS2 & ECM/STEM & YEATS domain containing 2 & {$[8.69-8.75-8.8]$} & {$[8.72-8.79-8.86]$} & {$[9.1-9.15-9.2]$} & {$[9.59-9.66-9.73]$} & $8.61 \mathrm{E}-36$ \\
\hline LCN2 & ECM/STEM & lipocalin 2 & {$[8.66-8.87-9.08]$} & {$[10.6-10.84-11.09]$} & {$[9.98-10.19-10.41]$} & {$[9.39-9.64-9.89]$} & $5.34 \mathrm{E}-38$ \\
\hline LUM & ECM/STEM & lumican & {$[9.18-9.37-9.57]$} & {$[7.45-7.72-7.99]$} & {$[9.37-9.65-9.92]$} & {$[9.37-9.64-9.91]$} & $9.72 \mathrm{E}-44$ \\
\hline SLC52A2 & ECM/STEM & solute carrier family 52 (riboflavin transporter), member 2 & {$[8.92-8.98-9.04]$} & {$[9.02-9.1-9.18]$} & [ $9.26-9.34-9.42]$ & {$[9.54-9.63-9.72]$} & $5.86 \mathrm{E}-46$ \\
\hline GPD1L & ECM/STEM & glycerol-3-phosphate dehydrogenase 1-like & {$[8.66-8.75-8.84]$} & {$[8.41-8.52-8.63]$} & {$[9.23-9.32-9.41]$} & {$[9.5-9.61-9.71]$} & $7.01 \mathrm{E}-65$ \\
\hline MTHFD2 & ECM/STEM & $\begin{array}{l}\text { methylenetetrahydrofolate dehydrogenase (NADP+ dependent) } 2 \text {, } \\
\text { methenyltetrahydrofolate cyclohydrolase }\end{array}$ & {$[8.6-8.68-8.75]$} & {$[8.38-8.49-8.6]$} & {$[8.79-8.89-9]$} & {$[9.45-9.6-9.74$ ] } & $1.73 E-36$ \\
\hline TIMELESS & ECM/STEM & timeless homolog (Drosophila) & {$[9.3-9.36-9.43]$} & {$[9.1-9.17-9.25]$} & {$[9.18-9.25-9.32]$} & {$[9.48-9.56-9.64]$} & $4.79 \mathrm{E}-38$ \\
\hline \begin{tabular}{|l|l} 
TESC \\
\end{tabular} & ECM/STEM & tescalcin & {$[8.22-8.32-8.42]$} & {$[8.08-8.22-8.37]$} & {$[8.89-9.03-9.17]$} & {$[9.38-9.55-9.72]$} & $1.37 \mathrm{E}-46$ \\
\hline PLEKHB2 & ECM/STEM & pleckstrin homology domain containing, family B (evectins) member 2 & {$[8.85-8.91-8.97$ ] } & {$[8.92-9-9.08]$} & {$[9.11-9.17-9.24]$} & {$[9.43-9.51-9.59]$} & $2.70 \mathrm{E}-40$ \\
\hline RFC5 & ECMISTEM & replication factor C (activator 1) 5, 36.5kDa & {$[9.3-9.37-9.45]$} & {$[9.11-9.17-9.24]$} & {$[9.13-9.2-9.27]$} & {$[9.4-9.48-9.56]$} & $5.91 \mathrm{E}-47$ \\
\hline ARHGEF2 & ECM/STEM & Rho/Rac guanine nucleotide exchange factor (GEF) 2 & {$[8.35-8.42-8.48]$} & {$[8.32-8.4-8.49]$} & {$[8.88-8.95-9.03]$} & {$[9.4-9.48-9.56]$} & $3.44 \mathrm{E}-27$ \\
\hline TUBB3 & ECM/STEM & tubulin, beta 3 & {$[8.54-8.61-8.68]$} & {$[8.48-8.56-8.65]$} & {$[8.98-9.07-9.16]$} & {$[9.35-9.48-9.6]$} & $9.81 \mathrm{E}-22$ \\
\hline COLGALT1 & ECM/STEM & collagen beta(1-0)galactosyltransferase 1 & {$[9.22-9.27-9.32]$} & {$[9.1-9.17-9.23]$} & {$[9.19-9.25-9.31]$} & {$[9.4-9.48-9.55]$} & $7.94 \mathrm{E}-21$ \\
\hline ASNS & ECM/STEM & asparagine synthetase (glutamine-hydrolyzing) & {$[7.67-7.79-7.9]$} & {$[7.67-7.85-8.02]$} & {$[8.75-8.89-9.04]$} & {$[9.31-9.47-9.64]$} & $1.32 \mathrm{E}-49$ \\
\hline CLSTN1 & ECM/STEM & calsyntenin 1 & {$[8.75-8.82-8.89]$} & {$[8.73-8.83-8.92]$} & {$[9.03-9.1-9.17]$} & {$[9.36-9.45-9.54]$} & $2.40 \mathrm{E}-21$ \\
\hline MST4 & ECM/STEM & serine/threonine protein kinase MST4 & {$[8.91-9-9.09]$} & {$[8.33-8.49-8.65]$} & {$[9.28-9.38-9.48]$} & [9.34 - $9.45-9.56]$ & $6.88 \mathrm{E}-25$ \\
\hline CAPG & ECM/STEM & capping protein (actin filament), gelsolin-like & {$[8.17-8.28-8.38]$} & {$[7.78-7.92-8.06]$} & {$[8.9-9.02-9.15]$} & {$[9.3-9.44-9.57]$} & $5.54 \mathrm{E}-26$ \\
\hline KRT23 & ECM/STEM & keratin 23 (histone deacetylase inducible) & {$[7.85-8.04-8.23]$} & {$[7.85-8.11-8.36]$} & {$[9.2-9.43-9.65]$} & {$[9.16-9.43-9.7]$} & \begin{tabular}{|c|c|}
$1.14 \mathrm{E}-29$ \\
\end{tabular} \\
\hline MAPK13 & ECM/STEM & mitogen-activated protein kinase 13 & {$[7.55-7.65-7.75]$} & {$[7.14-7.25-7.36]$} & {$[8.44-8.57-8.7]$} & {$[9.26-9.41-9.55]$} & $5.09 \mathrm{E}-76$ \\
\hline UGCG & ECM/STEM & UDP-glucose ceramide glucosyltransferase & {$[9.13-9.2-9.28]$} & {$[8.59-8.7-8.8]$} & {$[9.15-9.23-9.31]$} & {$[9.3-9.39-9.48]$} & $3.20 \mathrm{E}-30$ \\
\hline PHF21A & ECM/STEM & PHD finger protein $21 \mathrm{~A}$ & {$[8.62-8.67-8.73]$} & {$[8.69-8.76-8.84]$} & {$[9.02-9.07-9.13]$} & {$[9.32-9.39-9.45]$} & $4.63 \mathrm{E}-62$ \\
\hline SOBP & ECM/STEM & sine oculis binding protein homolog (Drosophila) & {$[8.05-8.17-8.29]$} & {$[7.51-7.69-7.87]$} & {$[8.69-8.84-8.98]$} & {$[9.17-9.36-9.55]$} & $9.15 \mathrm{E}-45$ \\
\hline MTMR2 & ECM/STEM & myotubularin related protein 2 & {$[8.45-8.52-8.59]$} & {$[8.17-8.28-8.4]$} & {$[8.77-8.85-8.93]$} & {$[9.28-9.36-9.44]$} & $3.23 \mathrm{E}-28$ \\
\hline G6PC3 & ECM/STEM & glucose 6 phosphatase, catalytic, 3 & {$[8.97-9.01-9.06]$} & {$[9.07-9.12-9.17]$} & {$[8.99-9.06-9.12]$} & {$[9.26-9.35-9.44]$} & $1.17 \mathrm{E}-42$ \\
\hline P2RX4 & ECM/STEM & purinergic receptor $\mathrm{P} 2 \mathrm{X}$, ligand-gated ion channel, 4 & {$[8.65-8.72-8.79]$} & {$[8.46-8.55-8.64]$} & {$[8.98-9.05-9.12]$} & [9.25 - $9.33-9.42]$ & $8.07 \mathrm{E}-34$ \\
\hline
\end{tabular}

Hepatology

This article is protected by copyright. All rights reserved. 


\begin{tabular}{|c|c|c|c|c|c|c|c|}
\hline RNF44 & ECM/STEM & ring finger protein 44 & {$[8.67-8.72-8.76]$} & {$[9.09-9.17-9.24]$} & {$[9.02-9.07-9.13]$} & {$[9.24-9.31-9.39]$} & $6.16 \mathrm{E}-30$ \\
\hline STX3 & ECM/STEM & syntaxin 3 & {$[8.27-8.34-8.41]$} & {$[8.22-8.34-8.45]$} & {$[8.69-8.79-8.88]$} & {$[9.19-9.3-9.4]$} & $5.37 \mathrm{E}-43$ \\
\hline H2AFY2 & ECM/STEM & H2A histone family, member Y2 & {$[7.72-7.82-7.92]$} & {$[8.19-8.34-8.5]$} & {$[8.73-8.86-8.99]$} & {$[9.14-9.3-9.46]$} & $8.42 \mathrm{E}-43$ \\
\hline ARID3A & ECM/STEM & AT rich interactive domain $3 \mathrm{~A}$ (BRIGHT-like) & {$[7.74-7.81-7.88]$} & {$[7.79-7.89-7.99]$} & {$[8.55-8.67-8.78]$} & {$[9.13-9.29-9.45]$} & $4.17 \mathrm{E}-27$ \\
\hline CDC42EP1 & ECM/STEM & CDC42 effector protein (Rho GTPase binding) 1 & {$[8.68-8.74-8.8]$} & {$[8.46-8.54-8.62]$} & {$[8.99-9.06-9.13]$} & {$[9.16-9.26-9.35]$} & $1.15 \mathrm{E}-69$ \\
\hline VNN2 & ECM/STEM & vanin 2 & {$[8.37-8.51-8.65]$} & {$[7.31-7.47-7.63]$} & {$[9.02-9.18-9.35]$} & {$[9.05-9.23-9.42]$} & $1.59 \mathrm{E}-62$ \\
\hline TRAF5 & ECM/STEM & TNF receptor-associated factor 5 & {$[7.97-8.06-8.15]$} & {$[8.05-8.17-8.29]$} & {$[8.77-8.87-8.97]$} & {$[9.11-9.23-9.34]$} & $3.95 \mathrm{E}-42$ \\
\hline POSTN & ECM/STEM & periostin, osteoblast specific factor & {$[7.81-7.98-8.15]$} & {$[7.21-7.41-7.61]$} & {$[8.58-8.78-8.98]$} & {$[9-9.22-9.45]$} & $1.68 \mathrm{E}-49$ \\
\hline TP53BP1 & ECM/STEM & tumor protein p53 binding protein 1 & {$[8.71-8.75-8.8]$} & {$[8.8-8.86-8.92]$} & {$[8.92-8.96-9.01]$} & {$[9.15-9.21-9.28]$} & $2.09 \mathrm{E}-38$ \\
\hline KDELR3 & ECM/STEM & $\begin{array}{l}\text { KDEL (Lys-Asp-Glu-Leu) endoplasmic reticulum protein retention receptor } \\
3\end{array}$ & {$[8.61-8.73-8.85]$} & {$[7.88-8.03-8.19]$} & {$[8.97-9.08-9.2]$} & {$[9.07-9.21-9.35]$} & $1.98 \mathrm{E}-41$ \\
\hline TACC3 & ECM/STEM & transforming, acidic coiled-coil containing protein 3 & {$[8.23-8.3-8.37]$} & {$[8.21-8.3-8.4]$} & {$[8.55-8.62-8.69]$} & {$[9.11-9.2-9.3]$} & $5.84 \mathrm{E}-68$ \\
\hline S100A9 & ECM/STEM & S100 calcium binding protein A9 & {$[8.03-8.15-8.28]$} & {$[7.6-7.76-7.92]$} & {$[8.42-8.56-8.7]$} & {$[8.96-9.2-9.44]$} & $1.66 \mathrm{E}-19$ \\
\hline DKK1 & ECM/STEM & dickkopf homolog 1 (Xenopus laevis) & {$[5.73-5.86-5.99]$} & {$[7.28-7.64-8.01]$} & {$[6.89-7.1-7.31]$} & {$[8.86-9.18-9.49]$} & $1.70 \mathrm{E}-35$ \\
\hline RAB15 & ECM/STEM & RAB15, member RAS onocogene family & {$[8.66-8.74-8.81]$} & {$[9.25-9.34-9.44]$} & {$[9.09-9.16-9.23]$} & {$[9.08-9.17-9.26]$} & 1.94E-71 \\
\hline UCHL1 & ECM/STEM & ubiquitin carboxyl-terminal esterase L1 (ubiquitin thiolesterase) & {$[7.8-7.92-8.03]$} & {$[7.43-7.58-7.73]$} & {$[8.2-8.35-8.5]$} & {$[8.92-9.17-9.42]$} & $2.77 \mathrm{E}-14$ \\
\hline LYN & ECM/STEM & v-yes-1 Yamaguchi sarcoma viral related oncogene homolog & {$[8.62-8.69-8.77]$} & {$[8.92-9.01-9.11]$} & {$[8.93-9.01-9.08]$} & {$[9.06-9.16-9.26]$} & $1.18 \mathrm{E}-45$ \\
\hline DLG5 & ECM/STEM & discs, large homolog 5 (Drosophila) & {$[8.24-8.32-8.4]$} & {$[9.07-9.16-9.26]$} & {$[8.68-8.75-8.82]$} & {$[9.04-9.13-9.22]$} & $2.90 \mathrm{E}-29$ \\
\hline GPX7 & ECM/STEM & glutathione peroxidase 7 & {$[8.21-8.32-8.44]$} & {$[7.76-7.89-8.03]$} & {$[8.55-8.66-8.77]$} & {$[8.99-9.13-9.27]$} & $9.60 \mathrm{E}-57$ \\
\hline NSMAF & ECM/STEM & neutral sphingomyelinase ( $\mathrm{N}-\mathrm{SMase}$ ) activation associated factor & {$[8.26-8.32-8.39]$} & {$[8.57-8.66-8.75]$} & {$[8.86-8.93-8.99]$} & {$[9.05-9.13-9.2]$} & $4.83 \mathrm{E}-25$ \\
\hline ZNF532 & ECM/STEM & zinc finger protein 532 & {$[8.07-8.13-8.2]$} & {$[7.89-7.97-8.05]$} & {$[8.55-8.64-8.72]$} & {$[9.03-9.13-9.22]$} & $1.12 \mathrm{E}-43$ \\
\hline STK17A & ECM/STEM & serine/threonine kinase 17a & {$[8.32-8.39-8.46]$} & {$[8.61-8.7-8.79]$} & {$[8.79-8.87-8.94]$} & {$[9.03-9.12-9.2]$} & $9.31 \mathrm{E}-10$ \\
\hline ADAM9 & ECM/STEM & ADAM metallopeptidase domain 9 & {$[8.18-8.25-8.33]$} & {$[8.49-8.58-8.68]$} & {$[8.34-8.42-8.5]$} & {$[8.98-9.09-9.19]$} & $2.32 \mathrm{E}-53$ \\
\hline GTF2IRD1 & ECM/STEM & GTF2I repeat domain containing 1 & {$[8.6-8.68-8.76]$} & {$[9.15-9.24-9.33]$} & {$[8.68-8.75-8.82]$} & {$[9-9.08-9.15]$} & $3.24 \mathrm{E}-42$ \\
\hline C1orf106 & ECM/STEM & chromosome 1 open reading frame 106 & {$[7.7-7.85-8.01]$} & {$[6.96-7.16-7.35]$} & {$[8.62-8.79-8.95]$} & {$[8.89-9.07-9.25]$} & $7.52 \mathrm{E}-66$ \\
\hline VRK1 & ECM/STEM & vaccinia related kinase 1 & {$[8.5-8.56-8.63]$} & {$[8.28-8.36-8.43]$} & {$[8.62-8.68-8.74]$} & {$[8.98-9.06-9.14]$} & $1.19 \mathrm{E}-45$ \\
\hline BICC1 & ECM/STEM & bicaudal C homolog 1 (Drosophila) & {$[7.38-7.5-7.63]$} & {$[7.06-7.25-7.45]$} & {$[8.26-8.4-8.55]$} & {$[8.83-9.01-9.18]$} & $5.05 \mathrm{E}-64$ \\
\hline TPM4 & ECM/STEM & tropomyosin 4 & {$[8.1-8.17-8.23]$} & {$[7.94-8.04-8.15]$} & {$[8.43-8.51-8.6]$} & {$[8.85-8.97-9.09]$} & $5.02 \mathrm{E}-34$ \\
\hline MMP12 & ECM/STEM & matrix metallopeptidase 12 (macrophage elastase) & {$[5.95-6.1-6.25]$} & {$[6.59-6.82-7.04]$} & {$[7.34-7.56-7.78]$} & {$[8.66-8.95-9.24]$} & $5.79 \mathrm{E}-18$ \\
\hline CEP55 & ECM/STEM & centrosomal protein $55 \mathrm{kDa}$ & {$[7.44-7.52-7.61]$} & {$[7.48-7.59-7.71]$} & {$[8.06-8.15-8.24]$} & {$[8.83-8.95-9.07]$} & $2.24 \mathrm{E}-34$ \\
\hline PDGFRA & ECM/STEM & platelet-derived growth factor receptor, alpha polypeptide & {$[8.24-8.37-8.5]$} & {$[7.15-7.32-7.49]$} & {$[8.44-8.62-8.8]$} & {$[8.72-8.92-9.12]$} & $1.11 \mathrm{E}-48$ \\
\hline DSG2 & ECM/STEM & desmoglein 2 & {$[8.33-8.42-8.52]$} & {$[7.53-7.69-7.84]$} & {$[8.43-8.53-8.64]$} & {$[8.78-8.91-9.05]$} & $8.79 \mathrm{E}-22$ \\
\hline TCF7L1 & ECM/STEM & transcription factor 7-like 1 (T-cell specific, HMG-box) & {$[8.98-9.05-9.11]$} & {$[8.14-8.23-8.33]$} & {$[8.74-8.82-8.9]$} & {$[8.81-8.91-9.01]$} & $1.13 \mathrm{E}-47$ \\
\hline SPHK1 & ECM/STEM & sphingosine kinase 1 & {$[7.41-7.5-7.6]$} & {$[7.22-7.37-7.52]$} & {$[7.94-8.07-8.19]$} & {$[8.73-8.89-9.05]$} & $8.91 \mathrm{E}-59$ \\
\hline HK2 & ECM/STEM & hexokinase 2 & {$[7.3-7.39-7.48]$} & {$[7.22-7.34-7.46]$} & {$[7.96-8.1-8.24]$} & {$[8.68-8.85-9.03]$} & $3.30 \mathrm{E}-37$ \\
\hline RIT1 & ECM/STEM & Ras-like without CAAX 1 & {$[8.28-8.34-8.39]$} & {$[8.28-8.34-8.41]$} & {$[8.46-8.53-8.6]$} & {$[8.76-8.85-8.95]$} & $4.85 \mathrm{E}-25$ \\
\hline NDUFA4L2 & ECM/STEM & NADH dehydrogenase (ubiquinone) 1 alpha subcomplex, 4-like 2 & {$[8.06-8.13-8.21]$} & {$[8.01-8.09-8.18]$} & {$[8.26-8.36-8.46]$} & {$[8.7-8.85-9]$} & $1.11 \mathrm{E}-15$ \\
\hline SALL2 & ECM/STEM & sal-like 2 (Drosophila) & {$[7.24-7.35-7.46]$} & {$[6.91-7.05-7.19]$} & {$[8.1-8.24-8.38]$} & {$[8.67-8.85-9.02]$} & $2.48 \mathrm{E}-29$ \\
\hline ARHGEF3 & ECM/STEM & Rho guanine nucleotide exchange factor (GEF) 3 & {$[8.16-8.24-8.31]$} & {$[8.09-8.21-8.34]$} & {$[8.69-8.78-8.88]$} & {$[8.75-8.84-8.93]$} & $3.12 \mathrm{E}-54$ \\
\hline SULF1 & ECM/STEM & sulfatase 1 & {$[7.54-7.68-7.82]$} & {$[6.89-7.05-7.22]$} & {$[8.51-8.72-8.92]$} & {$[8.62-8.84-9.06]$} & $2.65 \mathrm{E}-39$ \\
\hline STEAP1 & ECM/STEM & six transmembrane epithelial antigen of the prostate 1 & {$[8.08-8.23-8.38]$} & {$[8.79-8.99-9.19]$} & {$[8.39-8.55-8.71]$} & {$[8.67-8.84-9.01]$} & $1.31 \mathrm{E}-30$ \\
\hline CENPA & ECM/STEM & centromere protein A & {$[7.77-7.87-7.97]$} & {$[7.74-7.85-7.97]$} & {$[8.08-8.17-8.27]$} & {$[8.73-8.83-8.93]$} & $1.57 \mathrm{E}-55$ \\
\hline
\end{tabular}




\begin{tabular}{|c|c|c|c|c|c|c|c|}
\hline SLC25A24 & ECM/STEM & $\begin{array}{l}\text { solute carrier family } 25 \text { (mitochondrial carrier; phosphate carrier), member } \\
24\end{array}$ & {$[8.13-8.22-8.31]$} & {$[7.81-7.95-8.08]$} & {$[8.33-8.44-8.55]$} & {$[8.68-8.82-8.96]$} & $4.98 \mathrm{E}-39$ \\
\hline G6PD & ECM/STEM & glucose-6-phosphate dehydrogenase & {$[7.35-7.43-7.52]$} & {$[7.75-7.89-8.02]$} & {$[7.95-8.05-8.15]$} & {$[8.66-8.8-8.95]$} & $4.50 \mathrm{E}-71$ \\
\hline SLC12A8 & ECM/STEM & solute carrier family 12 (potassium/chloride transporters), member 8 & {$[8.15-8.24-8.33]$} & {$[7.5-7.63-7.75]$} & {$[8.53-8.63-8.73]$} & {$[8.69-8.8-8.91]$} & $2.79 \mathrm{E}-67$ \\
\hline C12orf49 & ECM/STEM & chromosome 12 open reading frame 49 & {$[7.91-7.97-8.03]$} & {$[7.79-7.88-7.98]$} & {$[8.3-8.37-8.44]$} & {$[8.67-8.76-8.85]$} & $3.18 \mathrm{E}-69$ \\
\hline HILPDA & ECM/STEM & hypoxia inducible lipid droplet-associated & {$[7.83-7.9-7.97]$} & {$[7.82-7.91-8]$} & {$[7.96-8.03-8.11]$} & {$[8.6-8.72-8.84]$} & $4.33 \mathrm{E}-15$ \\
\hline TRIP13 & ECM/STEM & thyroid hormone receptor interactor 13 & {$[7.54-7.63-7.73]$} & {$[7.66-7.79-7.91]$} & {$[7.88-7.96-8.05]$} & {$[8.6-8.72-8.84]$} & $7.44 \mathrm{E}-38$ \\
\hline PSRC1 & ECM/STEM & proline/serine-rich coiled-coil 1 & {$[7.93-7.99-8.05]$} & {$[8.05-8.13-8.21]$} & {$[8.15-8.23-8.3]$} & {$[8.62-8.71-8.81]$} & $6.52 \mathrm{E}-51$ \\
\hline AGPAT5 & ECM/STEM & $\begin{array}{l}\text { 1-acylglycerol-3-phosphate 0-acyltransferase } 5 \text { (lysophosphatidic acid } \\
\text { acyltransferase, epsilon) }\end{array}$ & {$[8.72-8.83-8.93]$} & {$[8.05-8.17-8.29]$} & {$[8.49-8.58-8.67$ ] } & {$[8.58-8.7-8.81]$} & $5.26 \mathrm{E}-47$ \\
\hline MUC13 & ECM/STEM & mucin 13, cell surface associated & {$[8.61-8.79-8.96]$} & {$[7.02-7.21-7.4]$} & {$[8.97-9.19-9.41]$} & {$[8.42-8.68-8.95]$} & $2.42 \mathrm{E}-52$ \\
\hline SMOX & ECM/STEM & spermine oxidase & {$[7.66-7.72-7.78]$} & {$[7.68-7.78-7.87]$} & {$[8.07-8.15-8.23]$} & {$[8.57-8.67-8.77]$} & $1.09 \mathrm{E}-81$ \\
\hline DLGAP5 & ECM/STEM & discs, large (Drosophila) homolog-associated protein 5 & {$[7.24-7.34-7.45]$} & {$[7.33-7.47-7.6]$} & {$[7.76-7.87-7.98]$} & {$[8.54-8.67-8.81]$} & $2.31 \mathrm{E}-48$ \\
\hline BLM & ECM/STEM & Bloom syndrome, RecQ helicase-like & {$[7.57-7.66-7.76]$} & {$[7.29-7.4-7.51]$} & {$[8.04-8.14-8.23]$} & {$[8.55-8.66-8.77]$} & $2.69 \mathrm{E}-40$ \\
\hline ASAP1 & ECM/STEM & ArfGAP with SH3 domain, ankyrin repeat and PH domain 1 & {$[7.52-7.59-7.67]$} & {$[7.9-8.02-8.14]$} & {$[8.18-8.27-8.35]$} & {$[8.54-8.66-8.77]$} & $3.12 \mathrm{E}-30$ \\
\hline CDC7 & ECM/STEM & cell division cycle 7 homolog (S. cerevisiae) & {$[7.94-8.03-8.12]$} & {$[7.62-7.71-7.81]$} & {$[8.18-8.27-8.36]$} & {$[8.54-8.64-8.74]$} & $1.37 \mathrm{E}-15$ \\
\hline SLC4A7 & ECM/STEM & solute carrier family 4, sodium bicarbonate cotransporter, member 7 & {$[7.9-7.97-8.04]$} & {$[7.6-7.7-7.8]$} & {$[8.12-8.21-8.29]$} & {$[8.46-8.56-8.66$ ] } & $7.83 \mathrm{E}-72$ \\
\hline BUB1B & ECM/STEM & budding uninhibited by benzimidazoles 1 homolog beta (yeast) & {$[7.33-7.42-7.52]$} & {$[7.37-7.48-7.59]$} & {$[7.69-7.78-7.87]$} & {$[8.38-8.49-8.61]$} & $4.78 \mathrm{E}-54$ \\
\hline KIF2C & ECM/STEM & kinesin family member $2 \mathrm{C}$ & {$[7.37-7.45-7.54]$} & {$[7.42-7.53-7.63]$} & {$[7.79-7.88-7.97]$} & {$[8.37-8.47-8.58]$} & $3.01 \mathrm{E}-46$ \\
\hline KIF11 & ECM/STEM & kinesin family member 11 & {$[7.16-7.25-7.35]$} & {$[7.32-7.43-7.54]$} & {$[7.58-7.68-7.79]$} & {$[8.34-8.46-8.57]$} & $1.84 \mathrm{E}-45$ \\
\hline F2RL1 & ECM/STEM & coagulation factor II (thrombin) receptor-like 1 & {$[8.51-8.66-8.8]$} & {$[7.25-7.43-7.61]$} & {$[8.67-8.83-8.98]$} & {$[8.28-8.45-8.63]$} & $1.25 \mathrm{E}-75$ \\
\hline SHCBP1 & ECM/STEM & SHC SH2-domain binding protein 1 & {$[7.56-7.65-7.73]$} & {$[7.71-7.81-7.91]$} & {$[7.93-8.02-8.1]$} & {$[8.32-8.43-8.54]$} & $8.28 \mathrm{E}-35$ \\
\hline KIF4A & ECM/STEM & kinesin family member $4 \mathrm{~A}$ & {$[7.34-7.43-7.53]$} & {$[7.3-7.44-7.58]$} & {$[7.7-7.81-7.93]$} & {$[8.28-8.42-8.56]$} & $4.19 \mathrm{E}-45$ \\
\hline GNAZ & ECM/STEM & guanine nucleotide binding protein ( $G$ protein), alpha z polypeptide & {$[7.78-7.89-7.99]$} & {$[7.38-7.49-7.6]$} & {$[8.24-8.35-8.46]$} & {$[8.3-8.42-8.54$ ] } & $3.61 \mathrm{E}-31$ \\
\hline STK39 & ECM/STEM & serine threonine kinase 39 & {$[7.25-7.35-7.45]$} & {$[7.42-7.57-7.71]$} & {$[8.19-8.29-8.39]$} & {$[8.27-8.41-8.55]$} & $2.34 \mathrm{E}-08$ \\
\hline MGAT4A & ECM/STEM & $\begin{array}{l}\text { mannosyl (alpha-1,3-)-glycoprotein beta-1,4-N- } \\
\text { acetylglucosaminyltransferase, isozyme A }\end{array}$ & {$[7.85-7.92-8]$} & {$[7.58-7.69-7.79]$} & {$[8.21-8.28-8.34$ ] } & {$[8.31-8.4-8.5]$} & $3.22 \mathrm{E}-51$ \\
\hline SFI1 & ECM/STEM & Sfi1 homolog, spindle assembly associated (yeast) & {$[7.64-7.69-7.74]$} & {$[7.51-7.58-7.66]$} & {$[7.93-8-8.07]$} & {$[8.3-8.4-8.5]$} & 1.77E-27 \\
\hline BUB1 & ECM/STEM & budding uninhibited by benzimidazoles 1 homolog (yeast) & {$[6.92-7.02-7.13]$} & {$[7.14-7.28-7.42]$} & {$[7.44-7.55-7.67]$} & {$[8.26-8.39-8.51]$} & $7.51 \mathrm{E}-50$ \\
\hline EPS8L3 & ECM/STEM & EPS8-like 3 & {$[6.77-6.89-7]$} & {$[7.92-8.12-8.32]$} & {$[7.84-7.98-8.13]$} & {$[8.2-8.37-8.54]$} & $9.11 \mathrm{E}-55$ \\
\hline KIF23 & ECM/STEM & kinesin family member 23 & {$[6.99-7.09-7.19]$} & {$[6.91-7.02-7.13]$} & {$[7.31-7.41-7.5]$} & {$[8.19-8.31-8.43]$} & $2.50 \mathrm{E}-51$ \\
\hline SSUH2 & ECM/STEM & ssu-2 homolog (C. elegans) & {$[7.07-7.21-7.35]$} & {$[7.68-7.9-8.12]$} & {$[8.1-8.28-8.47]$} & {$[8.04-8.25-8.46]$} & $8.93 \mathrm{E}-25$ \\
\hline CENPE & ECM/STEM & centromere protein $\mathrm{E}, 312 \mathrm{kDa}$ & {$[7.02-7.12-7.22]$} & {$[7.07-7.19-7.31]$} & {$[7.43-7.53-7.64]$} & {$[8.11-8.24-8.37]$} & $2.71 \mathrm{E}-43$ \\
\hline ASRGL1 & ECM/STEM & asparaginase like 1 & {$[7.58-7.69-7.8]$} & {$[6.95-7.06-7.16]$} & {$[7.64-7.76-7.88]$} & {$[7.99-8.14-8.28]$} & $3.10 \mathrm{E}-32$ \\
\hline MEP1A & ECM/STEM & meprin A, alpha (PABA peptide hydrolase) & {$[6.32-6.53-6.74]$} & {$[5.52-5.68-5.84]$} & {$[7.17-7.41-7.64]$} & {$[7.79-8.09-8.39]$} & $3.09 \mathrm{E}-31$ \\
\hline SLC7A1 & ECM/STEM & $\begin{array}{l}\text { solute carrier family } 7 \text { (cationic amino acid transporter, } y+\text { system), } \\
\text { member } 1\end{array}$ & {$[6.65-6.73-6.82]$} & {$[6.84-6.96-7.09]$} & {$[7.43-7.52-7.61]$} & {$[7.9-8.01-8.12]$} & $3.59 \mathrm{E}-20$ \\
\hline SYNGR1 & ECM/STEM & synaptogyrin 1 & {$[7.25-7.36-7.47]$} & {$[7.92-8.08-8.23]$} & {$[7.83-7.95-8.07]$} & {$[7.76-7.9-8.03]$} & $1.42 \mathrm{E}-49$ \\
\hline HOMER1 & ECM/STEM & homer homolog 1 (Drosophila) & {$[6.9-7.04-7.17]$} & {$[6.26-6.43-6.6]$} & {$[7.47-7.6-7.72]$} & {$[7.65-7.78-7.92]$} & $2.55 \mathrm{E}-44$ \\
\hline $\mathrm{COCH}$ & ECM/STEM & coagulation factor $\mathrm{C}$ homolog, cochlin (Limulus polyphemus) & {$[7.28-7.47-7.65]$} & {$[6.11-6.29-6.48]$} & {$[7.59-7.78-7.96]$} & {$[7.57-7.78-7.99]$} & $2.94 \mathrm{E}-33$ \\
\hline
\end{tabular}

Hepatology

This article is protected by copyright. All rights reserved. 


\begin{tabular}{|c|c|c|c|c|c|c|c|}
\hline PLXNC1 & ECM/STEM & plexin C1 & {$[7.44-7.54-7.64]$} & {$[6.58-6.69-6.81]$} & {$[7.66-7.75-7.85]$} & {$[7.6-7.71-7.83]$} & $2.31 \mathrm{E}-62$ \\
\hline NRCAM & ECM/STEM & neuronal cell adhesion molecule & {$[6.12-6.27-6.43]$} & {$[7.12-7.44-7.75]$} & {$[7.29-7.48-7.66]$} & {$[7.36-7.57-7.78]$} & $1.42 \mathrm{E}-08$ \\
\hline LRRC8B & ECM/STEM & leucine rich repeat containing 8 family, member $\mathrm{B}$ & {$[6.8-6.88-6.95]$} & {$[6.37-6.47-6.58]$} & {$[7.13-7.22-7.31]$} & {$[7.33-7.45-7.57]$} & $7.62 \mathrm{E}-23$ \\
\hline APOA1 & PERIPORTAL & apolipoprotein A-I & {$[14.26-14.31-14.37]$} & [ $13.27-13.42-13.58$ ] & {$[13.66-13.77-13.89]$} & [ $12.64-12.87-13.11]$ & $4.32 \mathrm{E}-89$ \\
\hline HRG & PERIPORTAL & histidine-rich glycoprotein & {$[14.23-14.28-14.34]$} & [ 12.72 - 12.94 - 13.17 ] & {$[13.34-13.46-13.58]$} & [ $11.28-11.52-11.77]$ & $6.81 \mathrm{E}-113$ \\
\hline ALDOB & PERIPORTAL & aldolase B, fructose-bisphosphate & [ $14.15-14.2-14.25$ ] & {$[12.76-12.93-13.1]$} & {$[13.61-13.68-13.75]$} & [ $11.58-11.82-12.06$ ] & 7.62E-55 \\
\hline HPX & PERIPORTAL & hemopexin & [ $13.95-14-14.06$ ] & [ $13.19-13.32$ - 13.45] & {$[13.33-13.43-13.52]$} & [ $11.85-12.04-12.23$ ] & $2.20 \mathrm{E}-76$ \\
\hline PLG & PERIPORTAL & plasminogen & [ $13.44-13.51-13.57$ ] & [ $13.26-13.38-13.49]$ & {$[12.66-12.76-12.87]$} & [ $11.12-11.35-11.59$ ] & 3.79E-95 \\
\hline CPS1 & PERIPORTAL & carbamoyl-phosphate synthase 1 , mitochondrial & [ $13.33-13.42$ - 13.51$]$ & {$[12.93-13.11-13.28]$} & {$[12.6-12.75-12.9]$} & {$[10.84-11.1-11.36$ ] } & $2.87 \mathrm{E}-48$ \\
\hline ARG1 & PERIPORTAL & arginase, liver & [ $13.33-13.41-13.48]$ & {$[11.88-12.07-12.25]$} & {$[12.75-12.85-12.96$ ] } & [ $10.92-11.15-11.37]$ & $3.81 \mathrm{E}-47$ \\
\hline AGXT & PERIPORTAL & alanine-glyoxylate aminotransferase & [ $13.28-13.34-13.41]$ & {$[12.18-12.34-12.5]$} & {$[12.59-12.69-12.79]$} & [ $10.77-11.01-11.24$ ] & $3.09 \mathrm{E}-90$ \\
\hline F9 & PERIPORTAL & coagulation factor IX & [ $13.19-13.27-13.36$ ] & [ $11.67-11.88-12.09$ ] & [ $12.24-12.36-12.48]$ & [ $9.77-10.04-10.32$ ] & $8.12 \mathrm{E}-65$ \\
\hline $\mathrm{ADH} 1 \mathrm{~A}$ & PERIPORTAL & alcohol dehydrogenase $1 \mathrm{~A}$ (class I), alpha polypeptide & [ $13.15-13.25$ - 13.35] & {$[13.08-13.25-13.42]$} & {$[12.96-13.07-13.17]$} & {$[11.26-11.51-11.75]$} & $6.34 \mathrm{E}-76$ \\
\hline DCXR & PERIPORTAL & dicarbonyl/L-xylulose reductase & [ $13.14-13.2-13.26]$ & [ $13.49-13.57-13.65$ ] & {$[12.2-12.28-12.35]$} & [ $11.21-11.32-11.44]$ & $8.45 \mathrm{E}-86$ \\
\hline ASS1 & PERIPORTAL & argininosuccinate synthase 1 & [ $12.97-13.04-13.12$ ] & [ $12.56-12.64-12.71]$ & {$[12.43-12.5-12.56$ ] } & [ $11.76-11.87-11.98]$ & $2.56 \mathrm{E}-73$ \\
\hline BHMT & PERIPORTAL & betaine--homocysteine S-methyltransferase & {$[12.9-13-13.11]$} & [ $12.41-12.55-12.69$ ] & {$[11.74-11.88-12.01]$} & [ $9.97-10.18-10.4]$ & $5.33 \mathrm{E}-108$ \\
\hline SLC10A1 & PERIPORTAL & solute carrier family 10 (sodium/bile acid cotransporter family), member 1 & [ $12.65-12.73-12.82]$ & [ $11.49-11.68-11.86$ ] & {$[11.2$ - 11.34 - 11.48 ] } & {$[8.17-8.4-8.63]$} & $6.82 \mathrm{E}-23$ \\
\hline C6 & PERIPORTAL & complement component 6 & [ $12.64-12.73-12.82$ ] & [ $12.07-12.24-12.4$ ] & [ $11.92-12.03-12.14]$ & [ $10.15-10.34-10.53$ ] & 8.37E-97 \\
\hline APCS & PERIPORTAL & amyloid P component, serum & [ $12.62-12.72-12.82$ ] & [ $12.69-12.85-13.02$ ] & {$[12.34-12.49-12.64$ ] } & [ $10.71-10.97-11.23$ ] & $1.03 E-37$ \\
\hline HGD & PERIPORTAL & homogentisate 1,2-dioxygenase & [ $12.64-12.71-12.77$ ] & [ $12.61-12.71-12.81]$ & {$[11.91-11.99-12.08]$} & [ $10.83-10.97-11.11]$ & $3.10 \mathrm{E}-47$ \\
\hline FBP1 & PERIPORTAL & fructose-1,6-bisphosphatase 1 & {$[12.58-12.66-12.74]$} & [ $[11.11-11.26-11.42]$ & {$[11.92-12.01-12.1]$} & [ $10.67-10.81-10.94$ ] & $3.31 \mathrm{E}-130$ \\
\hline AZGP1 & PERIPORTAL & alpha-2-glycoprotein 1, zinc-binding & [ $12.59-12.65-12.71]$ & [ $12.38-12.47-12.55$ ] & {$[11.7-11.79-11.87]$} & {$[10.55-10.72-10.9]$} & 2.53E-141 \\
\hline ECHDC2 & PERIPORTAL & enoyl CoA hydratase domain containing 2 & {$[12.59-12.65-12.7]$} & {$[12.5-12.58-12.65]$} & {$[12.12-12.18-12.23]$} & {$[11.39-11.47-11.55]$} & $3.60 \mathrm{E}-41$ \\
\hline
\end{tabular}

Hepatology

This article is protected by copyright. All rights reserved. 


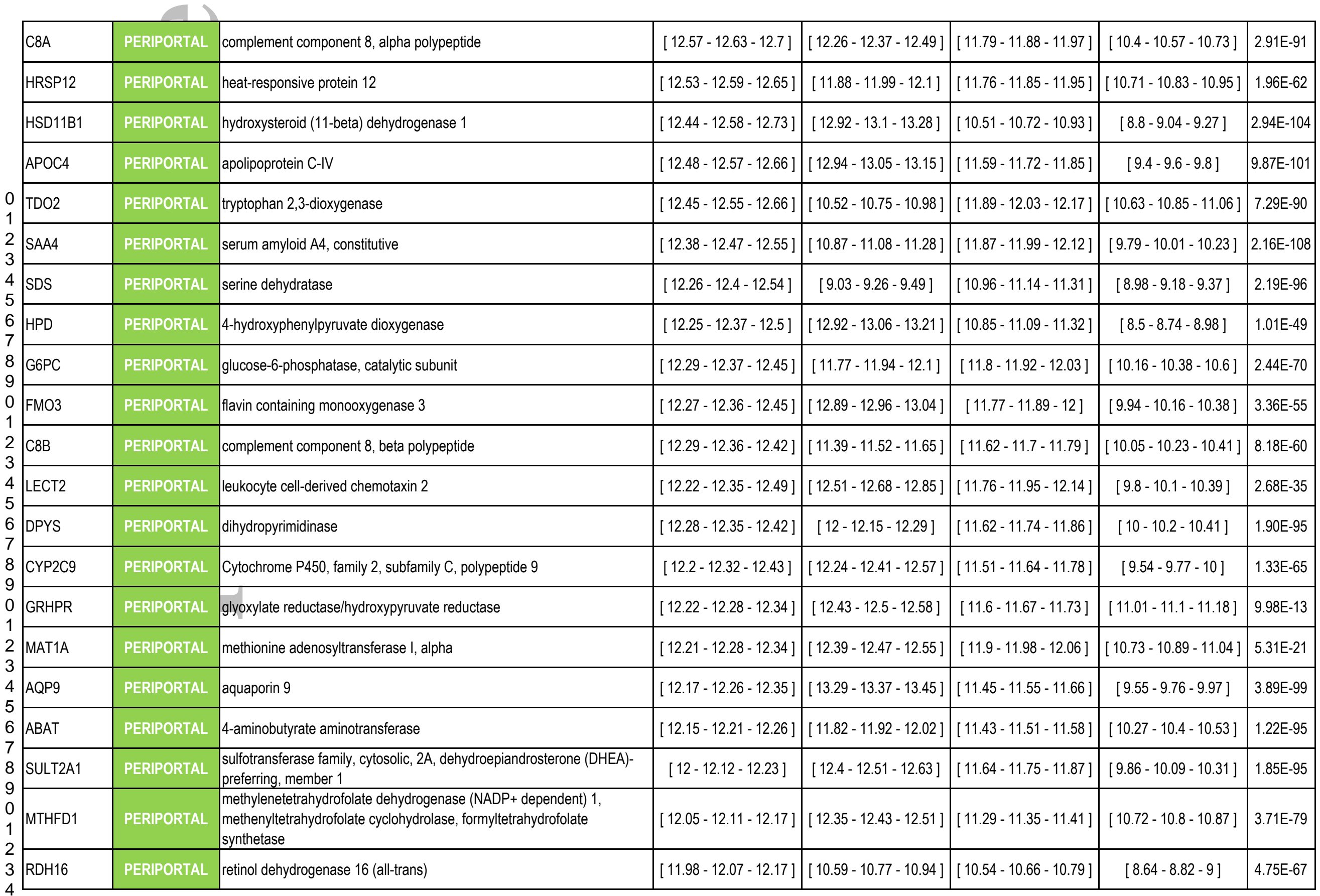

Hepatology

This article is protected by copyright. All rights reserved. 


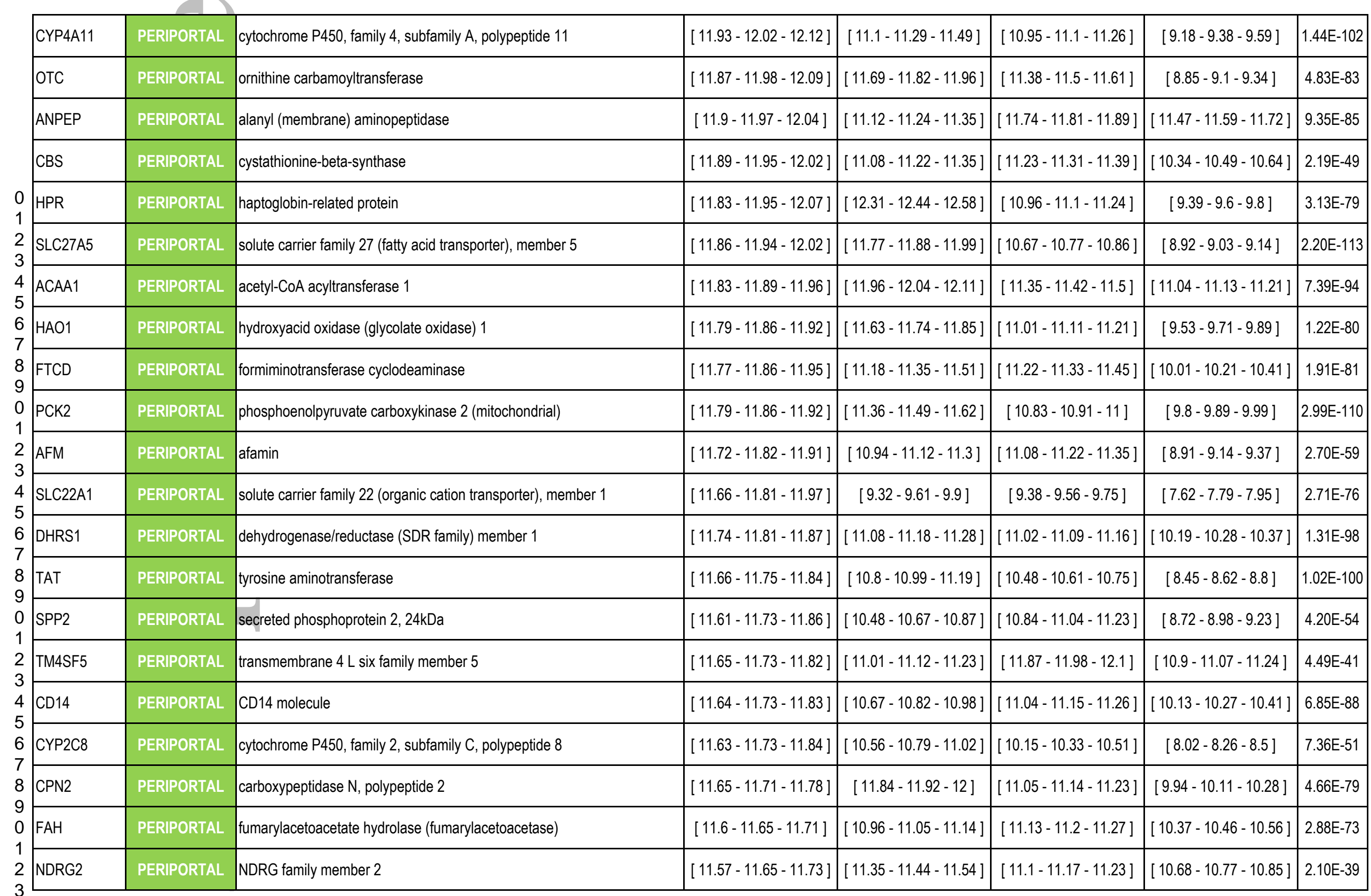

Hepatology

This article is protected by copyright. All rights reserved. 


\begin{tabular}{|c|c|c|c|c|c|c|c|}
\hline MTTP & PERIPORTAL & microsomal triglyceride transfer protein & [ $11.57-11.64-11.71]$ & {$[11.62-11.71-11.81]$} & {$[10.76-10.86-10.96]$} & {$[9.8-10.02-10.24]$} & $5.23 \mathrm{E}-70$ \\
\hline GADD45A & PERIPORTAL & growth arrest and DNA-damage-inducible, alpha & {$[11.56-11.63-11.69]$} & {$[11.45-11.53-11.61]$} & {$[10.97-11.03-11.09]$} & {$[10.45-10.53-10.6]$} & 2.42E-134 \\
\hline SLC22A7 & PERIPORTAL & solute carrier family 22 (organic anion transporter), member 7 & {$[11.51-11.61-11.71]$} & {$[9.45-9.67-9.9]$} & {$[10.86-10.98-11.11]$} & {$[8.93-9.15-9.38]$} & $2.54 \mathrm{E}-92$ \\
\hline ALDH8A1 & PERIPORTAL & aldehyde dehydrogenase 8 family, member A1 & {$[11.5-11.58-11.67]$} & {$[10.34-10.53-10.72]$} & {$[10.89-10.99-11.08$ ] } & {$[9.34-9.52-9.7]$} & $3.01 E-99$ \\
\hline CYP2J2 & PERIPORTAL & cytochrome P450, family 2, subfamily J, polypeptide 2 & [ $11.52-11.58-11.64$ ] & {$[11.36-11.45-11.54]$} & [ $10.78-10.85-10.93$ ] & {$[9.56-9.72-9.88]$} & $2.60 \mathrm{E}-33$ \\
\hline UPB1 & PERIPORTAL & ureidopropionase, beta & {$[11.49-11.58-11.66$ ] } & {$[11.13-11.26-11.39]$} & {$[11.01-11.12-11.23]$} & {$[9.17-9.4-9.62]$} & $1.30 \mathrm{E}-114$ \\
\hline СTH & PERIPORTAL & cystathionase (cystathionine gamma-lyase) & [ $11.45-11.56-11.67$ ] & {$[11.23-11.39-11.54$ ] } & {$[10.29-10.43-10.56$ ] } & {$[8.94-9.09-9.25]$} & 1.14E-98 \\
\hline AGXT2L1 & PERIPORTAL & alanine-glyoxylate aminotransferase 2-like 1 & {$[11.43-11.55-11.67$ ] } & {$[10.32-10.47-10.61]$} & {$[10.03-10.18-10.32]$} & {$[8.16-8.35-8.55]$} & $4.92 \mathrm{E}-90$ \\
\hline HAL & PERIPORTAL & histidine ammonia-lyase & {$[11.4-11.51-11.63]$} & {$[7.74-7.93-8.13]$} & {$[11.07-11.21-11.34]$} & {$[10.29-10.5-10.71]$} & $4.32 \mathrm{E}-101$ \\
\hline EPHX2 & PERIPORTAL & epoxide hydrolase 2 , cytoplasmic & {$[11.44-11.51-11.59]$} & {$[11.04-11.15-11.26]$} & [ 10.62 - $10.7-10.78]$ & {$[9.75-9.87-9.98]$} & 1.65E-119 \\
\hline SLC37A4 & PERIPORTAL & solute carrier family 37 (glucose-6-phosphate transporter), member 4 & {$[11.43-11.49-11.55]$} & {$[10.9-10.98-11.07]$} & {$[11.04-11.11-11.18]$} & {$[10.55-10.66-10.76]$} & 1.62E-51 \\
\hline ABCB4 & PERIPORTAL & ATP-binding cassette, sub-family B (MDR/TAP), member 4 & [ $11.37-11.46-11.56$ ] & {$[11.33-11.44-11.55]$} & {$[10.27-10.38-10.5]$} & {$[8.92-9.07-9.22]$} & $1.93 \mathrm{E}-39$ \\
\hline FETUB & PERIPORTAL & fetuin B & {$[11.35-11.44-11.53]$} & {$[9.51-9.78-10.04]$} & {$[10.04-10.18-10.33]$} & {$[7.98-8.15-8.33]$} & $3.49 \mathrm{E}-64$ \\
\hline C9 & PERIPORTAL & complement component 9 & {$[11.23-11.44-11.64$ ] } & {$[8.85-9.19-9.54]$} & {$[10.59-10.82-11.05]$} & {$[8.79-9.07-9.34]$} & $3.32 \mathrm{E}-18$ \\
\hline PON1 & PERIPORTAL & paraoxonase 1 & {$[11.33-11.43-11.53]$} & {$[10.63-10.82-11]$} & {$[10.42-10.54-10.67]$} & {$[8.63-8.84-9.05]$} & $1.75 \mathrm{E}-78$ \\
\hline RGN & PERIPORTAL & regucalcin (senescence marker protein-30) & {$[11.35-11.41-11.48]$} & {$[11.35-11.45-11.54$ ] } & {$[10.81-10.91-11]$} & {$[9.67-9.82-9.96]$} & 1.49E-47 \\
\hline ACSL1 & PERIPORTAL & acyl-CoA synthetase long-chain family member 1 & {$[11.26-11.33-11.41]$} & {$[10.76-10.87-10.98]$} & {$[10.72-10.81-10.91]$} & {$[9.75-9.9-10.04]$} & $2.28 \mathrm{E}-45$ \\
\hline $\mathrm{HAO} 2$ & PERIPORTAL & hydroxyacid oxidase 2 (long chain) & {$[11.19-11.32-11.46]$} & {$[8.79-9.02-9.25]$} & {$[9.51-9.68-9.84]$} & {$[7.53-7.71-7.88]$} & $4.97 \mathrm{E}-45$ \\
\hline CYP2C18 & PERIPORTAL & cytochrome P450, family 2, subfamily C, polypeptide 18 & {$[11.2-11.3-11.4]$} & {$[9.66-9.85-10.05]$} & {$[10.71-10.83-10.94]$} & {$[9.4-9.6-9.8]$} & $1.32 \mathrm{E}-79$ \\
\hline GNMT & PERIPORTAL & glycine N-methyltransferase & {$[11.1-11.25-11.4]$} & {$[9.69-9.95-10.2]$} & {$[9.72-9.88-10.03]$} & {$[8.09-8.26-8.44]$} & $4.03 \mathrm{E}-131$ \\
\hline |TIH4 & PERIPORTAL & $\begin{array}{l}\text { inter-alpha (globulin) inhibitor H4 (plasma Kallikrein-sensitive } \\
\text { glycoprotein) }\end{array}$ & {$[11.1-11.18-11.25]$} & {$[10.74-10.85-10.97]$} & {$[10.66-10.76-10.86]$} & {$[9.55-9.69-9.83]$} & $8.67 \mathrm{E}-74$ \\
\hline KLKB1 & PERIPORTAL & kallikrein B, plasma (Fletcher factor) 1 & {$[11.08-11.16-11.24]$} & {$[10.3-10.43-10.55]$} & {$[10.32-10.41-10.51]$} & {$[9.25-9.4-9.55]$} & $7.72 \mathrm{E}-92$ \\
\hline
\end{tabular}

Hepatology

This article is protected by copyright. All rights reserved. 


\begin{tabular}{|c|c|c|c|c|c|c|c|}
\hline GLS2 & PERIPORTAL & glutaminase 2 (liver, mitochondrial) & {$[11-11.15-11.3]$} & {$[6.86-7.07-7.28]$} & {$[9.25-9.4-9.54]$} & {$[7.37-7.53-7.69]$} & $3.19 \mathrm{E}-100$ \\
\hline MASP2 & PERIPORTAL & mannan-binding lectin serine peptidase 2 & {$[11.05-11.14-11.23]$} & [ $10.93-11.05-11.16$ ] & {$[10.11-10.25-10.4$ ] } & {$[8.49-8.66-8.83]$} & $5.15 \mathrm{E}-123$ \\
\hline GBA3 & PERIPORTAL & glucosidase, beta, acid 3 (cytosolic) & {$[11-11.11-11.23]$} & {$[8.84-9.05-9.25]$} & {$[9.24-9.41-9.58]$} & {$[7.85-8.06-8.26]$} & $4.67 \mathrm{E}-119$ \\
\hline AKR7A3 & PERIPORTAL & aldo-keto reductase family 7 , member A3 (aflatoxin aldehyde reductase) & [ $10.98-11.1-11.22$ ] & {$[10.42-10.59-10.75]$} & {$[9.81-9.98-10.16]$} & {$[8.33-8.51-8.68]$} & $1.77 \mathrm{E}-92$ \\
\hline PPP2R1B & PERIPORTAL & protein phosphatase 2 , regulatory subunit $A$, beta & {$[11.04-11.1-11.15]$} & {$[10.58-10.69-10.79]$} & {$[10.96-11.05-11.14]$} & {$[10.39-10.49-10.6]$} & $1.02 \mathrm{E}-79$ \\
\hline CYP4F2 & PERIPORTAL & cytochrome P450, family 4, subfamily F, polypeptide 2 & [ $10.98-11.07-11.17]$ & {$[10.63-10.76-10.88]$} & {$[10.16-10.27-10.38]$} & {$[8.59-8.76-8.92]$} & $1.21 \mathrm{E}-89$ \\
\hline EHHADH & PERIPORTAL & enoyl-CoA, hydratase/3-hydroxyacyl CoA dehydrogenase & {$[11-11.07-11.14]$} & [ $11.08-11.18-11.29]$ & {$[10.14-10.23-10.32]$} & {$[9.01-9.14-9.28]$} & 2.47E-24 \\
\hline NR113 & PERIPORTAL & nuclear receptor subfamily 1, group I, member 3 & [ $10.92-11.03-11.13]$ & {$[10.58-10.75-10.92$ ] } & {$[9.48-9.64-9.8]$} & {$[7.98-8.17-8.36$ ] } & $1.21 \mathrm{E}-85$ \\
\hline PCK1 & PERIPORTAL & phosphoenolpyruvate carboxykinase 1 (soluble) & {$[10.88-11-11.13]$} & [ $10.27-10.43-10.59]$ & {$[9.67-9.82$ - 9.97$]$} & {$[7.88-8.05-8.22]$} & $1.27 \mathrm{E}-84$ \\
\hline ETFDH & PERIPORTAL & electron-transferring-flavoprotein dehydrogenase & {$[10.9-10.97-11.04$ ] } & {$[10.67-10.76-10.85]$} & {$[10.31-10.37-10.43]$} & {$[9.61-9.71-9.81]$} & $1.69 \mathrm{E}-65$ \\
\hline ACACB & PERIPORTAL & acetyl-CoA carboxylase beta & [ $10.89-10.96-11.04$ ] & {$[9.98-10.07-10.17]$} & {$[10.43-10.51-10.59]$} & {$[9.53-9.66-9.78]$} & 3.14E-137 \\
\hline GABARAPL1 & PERIPORTAL & GABA(A) receptor-associated protein like 1 & [ $10.85-10.93-11.01]$ & {$[11.17-11.27-11.36]$} & {$[10.4-10.48-10.55]$} & {$[9.93-10.02-10.12$ ] } & $6.26 \mathrm{E}-114$ \\
\hline FXYD1 & PERIPORTAL & FXYD domain containing ion transport regulator 1 & [ $10.81-10.92-11.02$ ] & {$[8.74-8.98-9.21]$} & {$[9.64-9.78-9.91]$} & {$[8.54-8.72-8.89]$} & $1.80 \mathrm{E}-62$ \\
\hline GNE & PERIPORTAL & glucosamine (UDP-N-acetyl)-2-epimerase/N-acetylmannosamine kinase & {$[10.85-10.92-10.98]$} & {$[10.56-10.66-10.75]$} & {$[10-10.1-10.19]$} & {$[9.52-9.61-9.7]$} & $5.54 \mathrm{E}-90$ \\
\hline OGDHL & PERIPORTAL & oxoglutarate dehydrogenase-like & [ $10.79-10.89-10.98$ ] & {$[9.9-10.08-10.26]$} & {$[9.94-10.05-10.16]$} & {$[8.48-8.62-8.77$ ] } & $7.85 \mathrm{E}-43$ \\
\hline ADH6 & PERIPORTAL & alcohol dehydrogenase 6 (class V) & [ $10.79-10.88-10.97$ ] & {$[11.53-11.63-11.73]$} & {$[10.28-10.39-10.51]$} & {$[9.18-9.34-9.51]$} & $1.50 \mathrm{E}-85$ \\
\hline ALDH1L1 & PERIPORTAL & aldehyde dehydrogenase 1 family, member L1 & [ $10.75-10.86-10.98$ ] & {$[11.81-11.94-12.08]$} & {$[9.84-9.99-10.14$ ] } & {$[8.67-8.85-9.04$ ] } & $5.84 \mathrm{E}-115$ \\
\hline ABCA6 & PERIPORTAL & ATP-binding cassette, sub-family A (ABC1), member 6 & {$[10.78-10.86-10.94$ ] } & {$[10.74-10.85-10.96$ ] } & {$[9.89-9.99-10.09]$} & {$[8.62-8.79-8.95]$} & $6.31 \mathrm{E}-62$ \\
\hline AKR1D1 & PERIPORTAL & $\begin{array}{l}\text { aldo-keto reductase family 1, member D1 (delta 4-3-ketosteroid-5-beta- } \\
\text { reductase) }\end{array}$ & [ 10.68 - $10.82-10.97$ ] & {$[9.35-9.56-9.78]$} & {$[9.83-9.98-10.13]$} & {$[8.77-8.97-9.17$ ] } & 1.79E-40 \\
\hline GLYAT & PERIPORTAL & glycine-N-acyltransferase & {$[10.7-10.82-10.94$ ] } & {$[10.67-10.84-11.01]$} & {$[8.65-8.81-8.97]$} & {$[6.52-6.7-6.89]$} & 7.23E-95 \\
\hline ALDH2 & PERIPORTAL & aldehyde dehydrogenase 2 family (mitochondrial) & {$[10.75-10.8-10.85]$} & {$[10.76-10.83-10.91]$} & {$[10.37-10.43-10.5]$} & {$[9.86-9.93-10]$} & $2.59 \mathrm{E}-73$ \\
\hline PEMT & PERIPORTAL & phosphatidylethanolamine N-methyltransferase & {$[10.63-10.74-10.85]$} & {$[10.36-10.48-10.6]$} & {$[10.1-10.2-10.29]$} & {$[9.48-9.6-9.72]$} & $1.36 \mathrm{E}-125$ \\
\hline
\end{tabular}

Hepatology

This article is protected by copyright. All rights reserved. 


\begin{tabular}{|c|c|c|c|c|c|c|c|}
\hline SLC17A2 & PERIPORTAL & solute carrier family 17 (sodium phosphate), member 2 & [ $10.61-10.72$ - 10.83 ] & {$[10.86-11.01-11.17$ ] } & [ $10.22-10.35-10.49]$ & {$[9.22-9.43-9.64]$} & $2.06 \mathrm{E}-55$ \\
\hline APOF & PERIPORTAL & apolipoprotein F & {$[10.45-10.59-10.73$ ] } & {$[8.52-8.73-8.94]$} & {$[8.88-9.05-9.23]$} & {$[7.24-7.42-7.61]$} & $7.25 \mathrm{E}-56$ \\
\hline ACADSB & PERIPORTAL & acyl-CoA dehydrogenase, short/branched chain & {$[10.46-10.55-10.63$ ] } & {$[10.59-10.69-10.8]$} & {$[10.01-10.1-10.2]$} & {$[9.28-9.42-9.56]$} & $3.22 \mathrm{E}-109$ \\
\hline PBLD & PERIPORTAL & phenazine biosynthesis-like protein domain containing & {$[10.42-10.51-10.61]$} & {$[10.04-10.21-10.38]$} & {$[9.64-9.76-9.88]$} & {$[8.64-8.8-8.97$ ] } & 2.44E-29 \\
\hline SLCO1B1 & PERIPORTAL & solute carrier organic anion transporter family, member 1B1 & {$[10.39-10.49-10.58$ ] } & {$[10.17-10.33-10.49]$} & {$[9.95-10.08-10.2]$} & {$[8.27-8.5-8.73]$} & 2.69E-78 \\
\hline ANXA10 & PERIPORTAL & annexin A10 & {$[10.33-10.46-10.58]$} & {$[8.88-9.09-9.3]$} & {$[8.79-8.91-9.04]$} & {$[7.63-7.81-7.98]$} & 3.04E-111 \\
\hline ACSM5 & PERIPORTAL & acyl-CoA synthetase medium-chain family member 5 & {$[10.26-10.36-10.46$ ] } & {$[10.48-10.62-10.76]$} & {$[9.12-9.25-9.38]$} & {$[7.83-7.98-8.14]$} & $1.30 \mathrm{E}-101$ \\
\hline ABCA8 & PERIPORTAL & ATP-binding cassette, sub-family A (ABC1), member 8 & {$[10.16-10.28-10.4]$} & {$[9.97-10.15-10.34]$} & {$[9.14-9.3-9.47]$} & {$[7.95-8.17-8.39]$} & $1.30 \mathrm{E}-86$ \\
\hline$A B C C 6$ & PERIPORTAL & ATP-binding cassette, sub-family C (CFTR/MRP), member 6 & {$[10.17-10.25-10.32$ ] } & {$[10.24-10.32-10.4]$} & {$[9.8-9.89-9.98]$} & {$[9.23-9.35-9.47]$} & $2.60 \mathrm{E}-12$ \\
\hline CPE & PERIPORTAL & carboxypeptidase E & {$[10.13-10.24-10.36$ ] } & {$[9.07-9.19-9.32]$} & {$[10.27-10.39-10.51]$} & {$[9.89-10.04-10.2]$} & 2.16E-105 \\
\hline GCDH & PERIPORTAL & glutaryl-CoA dehydrogenase & {$[10.16-10.24-10.31]$} & {$[10.09-10.18-10.28]$} & [ $9.47-9.55-9.62]$ & {$[8.9-8.99-9.07]$} & $9.78 \mathrm{E}-21$ \\
\hline GHR & PERIPORTAL & growth hormone receptor & {$[10.1-10.21-10.31]$} & {$[8.91-9.05-9.2]$} & {$[9.23-9.35-9.46]$} & {$[8.09-8.23-8.38]$} & $2.05 \mathrm{E}-32$ \\
\hline SLC46A3 & PERIPORTAL & solute carrier family 46, member 3 & {$[10.07-10.19-10.3]$} & {$[9.46-9.64-9.83]$} & {$[8.89-9.02-9.16]$} & {$[7.89-8.02-8.16]$} & $9.41 \mathrm{E}-102$ \\
\hline ANO1 & PERIPORTAL & anoctamin 1 , calcium activated chloride channel & {$[10.02-10.15-10.27$ ] } & {$[8.66-8.89-9.12]$} & {$[8.83-8.99-9.16$ ] } & {$[8.02-8.17-8.32]$} & $6.60 \mathrm{E}-151$ \\
\hline SEC14L2 & PERIPORTAL & SEC14-like 2 (S. cerevisiae) & {$[10.04-10.13-10.22]$} & {$[10.26-10.37-10.49]$} & {$[8.76-8.85-8.94]$} & {$[7.59-7.7-7.8]$} & $3.53 E-59$ \\
\hline SLC1A1 & PERIPORTAL & $\begin{array}{l}\text { solute carrier family } 1 \text { (neuronal/epithelial high affinity glutamate } \\
\text { transporter, system Xag), member } 1\end{array}$ & {$[9.99-10.12-10.25]$} & {$[8.78-8.98-9.19]$} & {$[8.71-8.9-9.08]$} & {$[7.49-7.7-7.91]$} & $5.54 \mathrm{E}-43$ \\
\hline DNASE1L3 & PERIPORTAL & deoxyribonuclease l-like 3 & {$[9.97-10.1-10.24]$} & {$[9.6-9.75-9.91]$} & {$[9.07-9.21-9.35]$} & {$[8.3-8.45-8.61]$} & $2.78 \mathrm{E}-24$ \\
\hline GPR37 & PERIPORTAL & G protein-coupled receptor 37 (endothelin receptor type B-like) & {$[9.96-10.09-10.22]$} & {$[8.47-8.7-8.94]$} & {$[8.85-9.03-9.21]$} & {$[8.23-8.44-8.65]$} & $5.54 \mathrm{E}-54$ \\
\hline LRRC20 & PERIPORTAL & leucine rich repeat containing 20 & {$[10.02-10.08-10.15]$} & {$[9.89-9.97-10.05]$} & {$[9.99-10.07-10.15]$} & {$[9.3-9.4-9.5]$} & $4.81 \mathrm{E}-36$ \\
\hline $\mathrm{GCH} 1$ & PERIPORTAL & GTP cyclohydrolase 1 & {$[9.99-10.06-10.14]$} & {$[8.98-9.09-9.2]$} & {$[9.53-9.62-9.71]$} & {$[9.03-9.14-9.25]$} & $1.19 \mathrm{E}-101$ \\
\hline CYP2B7P1 & PERIPORTAL & cytochrome P450, family 2, subfamily B, polypeptide 7 pseudogene 1 & {$[9.89-10.05-10.2]$} & {$[7.32-7.51-7.69]$} & {$[8.34-8.51-8.69]$} & {$[7.22-7.38-7.53]$} & 7.44E-104 \\
\hline GYS2 & PERIPORTAL & glycogen synthase 2 (liver) & {$[9.92-10.03-10.15]$} & {$[8.92-9.11-9.3]$} & {$[8.07-8.2-8.34]$} & {$[6.33-6.5-6.66]$} & $5.49 \mathrm{E}-49$ \\
\hline CLDN15 & PERIPORTAL & claudin 15 & {$[9.93-10.03-10.12]$} & {$[9.82-9.94-10.06]$} & {$[9.67-9.77-9.87]$} & {$[8.91-9.03-9.14]$} & $5.28 \mathrm{E}-82$ \\
\hline KMO & PERIPORTAL & kynurenine 3-monooxygenase (kynurenine 3-hydroxylase) & {$[9.81-9.92-10.02]$} & {$[8.29-8.44-8.6]$} & {$[9.19-9.31-9.42]$} & {$[8.17-8.3-8.42]$} & $4.38 \mathrm{E}-23$ \\
\hline FEM1B & PERIPORTAL & fem-1 homolog b (C. elegans) & {$[9.86-9.9-9.95]$} & {$[9.55-9.62-9.68]$} & {$[9.76-9.81-9.85]$} & {$[9.67-9.73-9.78]$} & $4.58 \mathrm{E}-111$ \\
\hline ZGPAT & PERIPORTAL & zinc finger, $\mathrm{CCCH}$-type with $\mathrm{G}$ patch domain & {$[9.68-9.8-9.91]$} & {$[9.2-9.32-9.43]$} & {$[9.03-9.12-9.21]$} & {$[8.81-8.89-8.98]$} & $6.52 \mathrm{E}-158$ \\
\hline CSTA & PERIPORTAL & cystatin A (stefin A) & {$[9.65-9.77-9.89]$} & {$[8.5-8.69-8.87]$} & {$[9.39-9.53-9.67]$} & {$[9.58-9.74-9.89]$} & $1.66 \mathrm{E}-90$ \\
\hline NR112 & PERIPORTAL & nuclear receptor subfamily 1 , group I, member 2 & {$[9.67-9.76-9.85]$} & {$[8.91-9.06-9.21]$} & {$[8.29-8.43-8.57]$} & {$[7.14-7.29-7.44]$} & $1.88 \mathrm{E}-22$ \\
\hline
\end{tabular}

Hepatology

This article is protected by copyright. All rights reserved. 


\begin{tabular}{|c|c|c|c|c|c|c|c|}
\hline CDA & PERIPORTAL & cytidine deaminase & {$[9.65-9.74-9.84]$} & {$[8.32-8.45-8.58]$} & {$[8.99-9.1-9.21]$} & {$[8.35-8.5-8.65]$} & $3.70 \mathrm{E}-72$ \\
\hline LCAT & PERRIPORTAL & lecithin-cholesterol acyltransferase & {$[9.63-9.74-9.85]$} & {$[8.97-9.1-9.23]$} & {$[8.95-9.05-9.15]$} & {$[8.34-8.44-8.54]$} & $4.92 \mathrm{E}-83$ \\
\hline GPR88 & PERIPORTAL & G protein-coupled receptor 88 & {$[9.47-9.7-9.93]$} & {$[7.01-7.24-7.48]$} & {$[8.58-8.84-9.09]$} & {$[7.31-7.55-7.78]$} & $4.31 \mathrm{E}-18$ \\
\hline CRY1 & PERIPORTAL & cryptochrome 1 (photolyase-like) & {$[9.6-9.66-9.73]$} & {$[9.18-9.27-9.35]$} & {$[9.53-9.6-9.67]$} & {$[9.51-9.58-9.65]$} & $3.75 \mathrm{E}-103$ \\
\hline IGF1 & PERIPORTAL & insulin-like growth factor 1 (somatomedin C) & {$[9.48-9.62-9.77]$} & {$[8.58-8.76-8.95]$} & {$[8.31-8.47-8.63]$} & {$[7.74-7.87-8.01]$} & $2.63 \mathrm{E}-151$ \\
\hline SOCS2 & PERIPORTAL & suppressor of cytokine signaling 2 & {$[9.51-9.62-9.72]$} & {$[8.63-8.78-8.92]$} & {$[8.72-8.84-8.96]$} & {$[7.94-8.07-8.2]$} & $8.94 \mathrm{E}-67$ \\
\hline HMGB3 & PERIPORTAL & high-mobility group box 3 & {$[9.5-9.59-9.69]$} & {$[8.38-8.5-8.62]$} & {$[9.1-9.23-9.35]$} & {$[9.01-9.16-9.31]$} & $2.52 \mathrm{E}-33$ \\
\hline GSTZ1 & PERIPORTAL & glutathione transferase zeta 1 & {$[9.4-9.5-9.59]$} & {$[9.21-9.33-9.45]$} & {$[8.52-8.6-8.68]$} & {$[8.23-8.32-8.4]$} & $7.16 \mathrm{E}-54$ \\
\hline ACSM3 & PERIPORTAL & acyl-CoA synthetase medium-chain family member 3 & {$[9.34-9.44-9.54]$} & {$[9.5-9.6-9.71]$} & {$[8.29-8.4-8.51]$} & {$[7.93-8.04-8.16]$} & $2.03 \mathrm{E}-112$ \\
\hline PXMP4 & PERIPORTAL & peroxisomal membrane protein $4,24 \mathrm{kDa}$ & {$[9.36-9.43-9.5]$} & {$[9.22-9.3-9.39]$} & {$[9.17-9.24-9.32]$} & {$[8.8-8.88-8.96]$} & $6.23 \mathrm{E}-29$ \\
\hline GPN3 & PERIPORTAL & GPN-loop GTPase 3 & {$[9.36-9.41-9.47]$} & {$[9.09-9.15-9.22]$} & {$[9.07-9.15-9.22]$} & {$[9.01-9.08-9.15]$} & $2.82 \mathrm{E}-147$ \\
\hline MLLT11 & PERIPORTAL & $\begin{array}{l}\text { myeloid/lymphoid or mixed-lineage leukemia (trithorax homolog, } \\
\text { Drosophila); translocated to, } 11\end{array}$ & {$[9.26-9.4-9.54]$} & {$[8.07-8.21-8.36]$} & {$[8.82-8.96-9.11]$} & {$[8.95-9.11-9.26]$} & $1.28 \mathrm{E}-87$ \\
\hline GPD1 & PERRPORTAL & glycerol-3-phosphate dehydrogenase 1 (soluble) & {$[9.26-9.38-9.49]$} & {$[8.45-8.59-8.72]$} & {$[8.47-8.57-8.67]$} & {$[7.59-7.7-7.82]$} & $5.23 \mathrm{E}-117$ \\
\hline $\mathrm{BDH} 2$ & PERIPORTAL & 3-hydroxybutyrate dehydrogenase, type 2 & {$[9.23-9.33-9.43]$} & {$[8.39-8.5-8.62]$} & {$[8.65-8.76-8.86]$} & {$[8.24-8.34-8.45]$} & $5.61 \mathrm{E}-151$ \\
\hline NAT2 & PERIPORTAL & $\mathrm{N}$-acetyltransferase 2 (arylamine $\mathrm{N}$-acetyltransferase) & {$[9.13-9.3-9.46]$} & {$[8.96-9.14-9.31]$} & {$[7.66-7.81-7.97]$} & {$[6.69-6.83-6.96]$} & $1.50 \mathrm{E}-13$ \\
\hline SCGN & PERIPORTAL & secretagogin, EF-hand calcium binding protein & {$[9.09-9.27-9.45]$} & {$[7.56-7.73-7.9]$} & {$[8.47-8.65-8.83]$} & {$[8.38-8.57-8.77]$} & $4.59 \mathrm{E}-61$ \\
\hline CYP3A43 & PERIPORTAL & cytochrome P450, family 3 , subfamily A, polypeptide 43 & {$[9.06-9.21-9.36]$} & {$[9.35-9.51-9.67]$} & {$[8.29-8.43-8.57]$} & {$[7.11-7.25-7.38]$} & $1.82 \mathrm{E}-45$ \\
\hline ATP11B & PERIPORTAL & ATPase, class VI, type 11B & {$[9.08-9.14-9.2]$} & {$[8.61-8.69-8.77]$} & {$[9.01-9.09-9.16]$} & {$[9.11-9.19-9.27]$} & $1.51 \mathrm{E}-109$ \\
\hline CYFIP2 & PERIPORTAL & cytoplasmic FMR1 interacting protein 2 & {$[9.02-9.14-9.26]$} & {$[7.91-8.07-8.22]$} & {$[8.63-8.75-8.86]$} & {$[8.19-8.31-8.43]$} & $8.09 \mathrm{E}-41$ \\
\hline SRD5A2 & PERIPORTAL & $\begin{array}{l}\text { steroid-5-alpha-reductase, alpha polypeptide } 2 \text { (3-oxo-5 alpha-steroid } \\
\text { delta 4-dehydrogenase alpha 2) }\end{array}$ & {$[8.94-9.09-9.23]$} & {$[8.86-9.04-9.22]$} & {$[7.65-7.77-7.89]$} & {$[6.78-6.92-7.06]$} & $2.91 \mathrm{E}-69$ \\
\hline GRAMD1C & 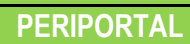 & GRAM domain containing $1 \mathrm{C}$ & {$[8.94-9.04-9.14]$} & {$[7.71-7.85-7.99]$} & {$[8.11-8.21-8.31]$} & {$[7.4-7.52-7.64]$} & $7.58 \mathrm{E}-111$ \\
\hline ACADL & PERIPORTAL & acyl-CoA dehydrogenase, long chain & {$[8.83-8.95-9.07]$} & {$[8.38-8.57-8.76]$} & {$[7.33-7.48-7.62]$} & {$[6.27-6.42-6.57]$} & $8.19 \mathrm{E}-62$ \\
\hline ESR1 & PERIPORTAL & estrogen receptor 1 & {$[8.72-8.84-8.97]$} & {$[8.41-8.61-8.81]$} & {$[7.37-7.49-7.6]$} & {$[6.95-7.05-7.16]$} & $1.33 \mathrm{E}-29$ \\
\hline CPEB3 & PERIPORTAL & cytoplasmic polyadenylation element binding protein 3 & {$[8.69-8.77-8.85]$} & {$[8.51-8.61-8.72]$} & {$[8.08-8.15-8.23]$} & {$[7.61-7.68-7.75]$} & $1.60 \mathrm{E}-99$ \\
\hline IGFALS & PERIPORTAL & insulin-like growth factor binding protein, acid labile subunit & {$[8.64-8.77-8.91]$} & {$[7.51-7.62-7.73]$} & {$[7.89-7.99-8.09]$} & {$[7.65-7.76-7.88]$} & $3.81 \mathrm{E}-31$ \\
\hline MNS1 & PERIPORTAL & meiosis-specific nuclear structural 1 & {$[8.59-8.74-8.89]$} & {$[7.6-7.72-7.84]$} & {$[7.86-7.99-8.11]$} & {$[7.56-7.66-7.75]$} & $4.23 \mathrm{E}-95$ \\
\hline FAM149A & PERIPORTAL & family with sequence similarity 149 , member A & {$[8.58-8.68-8.77]$} & {$[7.63-7.77-7.92]$} & {$[7.96-8.06-8.15]$} & {$[7.29-7.41-7.54]$} & $1.56 \mathrm{E}-36$ \\
\hline GNB5 & PERIPORTAL & guanine nucleotide binding protein ( $G$ protein), beta 5 & {$[8.52-8.61-8.7]$} & {$[7.61-7.71-7.81]$} & {$[8.31-8.41-8.51]$} & {$[7.87-7.97-8.07]$} & $1.60 \mathrm{E}-52$ \\
\hline VIPR1 & PERIPORTAL & vasoactive intestinal peptide receptor 1 & {$[8.41-8.53-8.65]$} & {$[7.85-7.99-8.13]$} & {$[7.73-7.84-7.95]$} & {$[7.26-7.37-7.47]$} & $8.44 \mathrm{E}-105$ \\
\hline KDM8 & PERIPORTAL & Lysine (K)-Specific Demethylase 8 & {$[8.38-8.5-8.62]$} & {$[7.76-7.87-7.98]$} & {$[7.78-7.87-7.96]$} & {$[7.34-7.42-7.51]$} & $1.24 \mathrm{E}-57$ \\
\hline LRRC31 & PERIPORTAL & leucine rich repeat containing 31 & {$[8.3-8.43-8.56]$} & {$[7.02-7.21-7.4]$} & {$[8.08-8.22-8.37]$} & {$[7.54-7.71-7.87]$} & $2.97 \mathrm{E}-78$ \\
\hline LPA & PERIPORTAL & lipoprotein, Lp(a) & {$[8.25-8.38-8.51]$} & {$[7.19-7.34-7.48]$} & {$[7.43-7.55-7.67]$} & {$[6.81-6.96-7.11]$} & $7.86 \mathrm{E}-81$ \\
\hline SLC4A4 & PERIPORTAL & solute carrier family 4 , sodium bicarbonate cotransporter, member 4 & {$[8.1-8.21-8.32]$} & {$[8.73-8.88-9.02]$} & {$[7.22-7.34-7.47]$} & {$[7.15-7.32-7.49]$} & $2.64 \mathrm{E}-112$ \\
\hline FAM134B & PERIPORTAL & family with sequence similarity 134 , member $B$ & {$[8.06-8.2-8.34]$} & {$[7.31-7.5-7.68]$} & {$[7.13-7.28-7.42]$} & {$[6.62-6.76-6.9]$} & $3.25 \mathrm{E}-26$ \\
\hline ADRA1A & PERIPORTAL & adrenergic, alpha-1A-, receptor & {$[7.99-8.13-8.27]$} & {$[7.55-7.73-7.91]$} & {$[6.95-7.07-7.2]$} & {$[6.22-6.34-6.46]$} & $1.73 \mathrm{E}-37$ \\
\hline AASS & PERIPORTAL & aminoadipate-semialdehyde synthase & {$[8-8.1-8.2]$} & {$[8.13-8.27-8.41]$} & {$[6.97-7.07-7.17]$} & {$[6.38-6.46-6.54]$} & $1.36 \mathrm{E}-30$ \\
\hline KCNN2 & PERIPORTAL & $\begin{array}{l}\text { potassium intermediate/small conductance calcium-activated channel, } \\
\text { subfamily N, member } 2\end{array}$ & {$[7.7-7.86-8.02]$} & {$[6.85-6.96-7.08]$} & {$[7.04-7.14-7.24$ ] } & {$[6.91-6.99-7.07]$} & $4.02 \mathrm{E}-100$ \\
\hline FOLH1B & PERIPORTAL & folate hydrolase 1B & {$[7.61-7.76-7.9]$} & {$[7-7.22-7.44]$} & {$[6.41-6.52-6.64]$} & {$[6.11-6.22-6.33]$} & $4.55 \mathrm{E}-50$ \\
\hline TENM1 & PERRIPORTAL & Teneurin Transmembrane Protein 1 & {$[7.41-7.59-7.76]$} & {$[6.38-6.6-6.82]$} & {$[6.37-6.52-6.67]$} & {$[6.46-6.64-6.83]$} & $3.04 \mathrm{E}-84$ \\
\hline
\end{tabular}

Hepatology

This article is protected by copyright. All rights reserved. 
Supporting Table S10. See Supplementary Data.docx file for caption.

\begin{tabular}{|c|c|c|c|c|c|}
\hline \multirow{2}{*}{ Datasets } & \multicolumn{4}{|c|}{ HCC subclasses } & \multirow{2}{*}{ Totals } \\
\cline { 2 - 5 } & PP & PV & ECM & STEM & $100(100 \%)$ \\
\hline A & $32(32 \%)$ & $19(19 \%)$ & $28(28 \%)$ & $21(21 \%)$ & $135(100 \%)$ \\
\hline B & $30(22 \%)$ & $27(20 \%)$ & $69(51 \%)$ & $9(7 \%)$ & $247(100 \%)$ \\
\hline C & $72(29 \%)$ & $27(11 \%)$ & $94(38 \%)$ & $54(22 \%)$ & $268(100 \%)$ \\
\hline D & $94(35 \%)$ & $50(19 \%)$ & $85(32 \%)$ & $39(15 \%)$ & $42(100 \%)$ \\
\hline E & $11(26 \%)$ & $14(33 \%)$ & $12(29 \%)$ & $5(12 \%)$ & $73(100 \%)$ \\
\hline F & $28(38 \%)$ & $9(12 \%)$ & $25(34 \%)$ & $11(15 \%)$ & $164(100 \%)$ \\
\hline G & $34(21 \%)$ & $37(23 \%)$ & $82(50 \%)$ & $11(7 \%)$ & $35(100 \%)$ \\
\hline H & $6(17 \%)$ & $12(34 \%)$ & $4(11 \%)$ & $13(37 \%)$ & $69(100 \%)$ \\
\hline I & $19(28 \%)$ & $15(22 \%)$ & $21(30 \%)$ & $14(20 \%)$ & 690 \\
\hline Totals & $326(29 \%)$ & $210(19 \%)$ & $420(37 \%)$ & $177(16 \%)$ & $1133(100 \%)$ \\
\hline
\end{tabular}




\section{Supporting Table S11. See Supplementary Data.docx file for caption.}

Sampling technique: metadata set, $n=1133$ HCCs

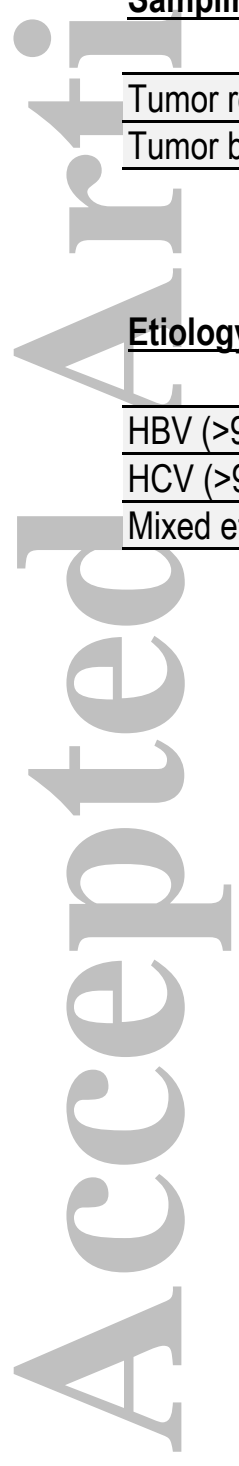




\section{Supporting Table S12. See Supplementary Data.docx file for caption.}

\begin{tabular}{|c|c|c|c|c|c|c|c|c|c|c|c|c|c|c|c|}
\hline $\mathrm{N}=210 \mathrm{HCCs}$ & $\overline{A G X T}$ & FETUB & GLS2 & GNMT & OTC & SLC10A1 & SLC22A7 & SLC27A5 & KRT19 & EPCAM & PROM1 & LGR5 & GLUL & ODAM & HAL \\
\hline FETUB & 0.66 & & & & & & & & & & & & & & \\
\hline GLS2 & 0.47 & 0.53 & & & & & & & & & & & & & \\
\hline GNMT & 0.58 & 0.56 & 0.52 & & & & & & & & & & & & \\
\hline OTC & 0.58 & 0.52 & 0.26 & 0.38 & & & & & & & & & & & \\
\hline SLC10A1 & 0.66 & 0.70 & 0.41 & 0.54 & 0.61 & & & & & & & & & & \\
\hline SLC22A7 & 0.60 & 0.59 & 0.32 & 0.24 & 0.44 & 0.53 & & & & & & & & & \\
\hline SLC27A5 & 0.49 & 0.56 & 0.26 & 0.42 & 0.66 & 0.77 & 0.36 & & & & & & & & \\
\hline KRT19 & -0.25 & -0.26 & -0.02 & -0.14 & -0.32 & -0.29 & -0.15 & -0.42 & & & & & & & \\
\hline EPCAM & -0.18 & -0.24 & 0.08 & -0.06 & -0.20 & -0.29 & -0.10 & -0.44 & 0.57 & & & & & & \\
\hline PROM1 & -0.36 & -0.38 & -0.12 & -0.25 & -0.32 & -0.38 & -0.24 & -0.52 & 0.67 & 0.54 & & & & & \\
\hline LGR5 & -0.17 & -0.25 & -0.46 & -0.09 & 0.15 & -0.15 & -0.23 & 0.04 & 0.01 & 0.10 & 0.11 & & & & \\
\hline GLUL & -0.04 & -0.05 & -0.54 & -0.02 & 0.29 & 0.20 & -0.04 & 0.34 & -0.25 & -0.19 & -0.15 & 0.65 & & & \\
\hline ODAM & -0.22 & -0.26 & -0.57 & -0.21 & 0.16 & -0.03 & -0.24 & 0.18 & -0.13 & -0.23 & -0.02 & 0.61 & 0.71 & & \\
\hline HAL & 0.43 & 0.35 & 0.71 & 0.32 & 0.08 & 0.16 & 0.35 & -0.11 & 0.15 & 0.27 & 0.07 & -0.48 & -0.66 & -0.67 & \\
\hline VNN1 & 0.16 & 0.16 & 0.40 & -0.04 & 0.04 & 0.00 & 0.27 & -0.25 & 0.22 & 0.25 & 0.18 & -0.42 & -0.56 & -0.51 & 0.61 \\
\hline
\end{tabular}

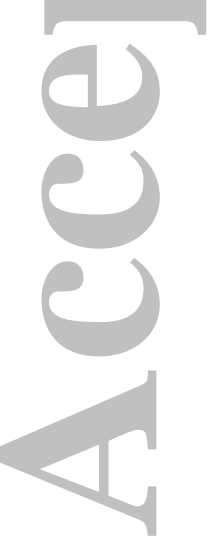

PERIPORTAL GENE SIGNATURE

\section{STEM CELL MARKERS}

\section{POSITIVE CTNNB1 MUTATION MARKERS}

NEGATIVE CTNNB1 MUTATION MARKERS 


\title{
Supporting Data to:
}

Human Hepatocellular Carcinomas with a Periportal Phenotype Have the

\section{Lowest Potential for Early Recurrence after Curative Resection}

Romain Désert, Florian Rohart, Frédéric Canal, Marie Sicard, Mireille Desille, Stéphanie Renaud, Bruno Turlin, Pascale Bellaud, Christine Perret, Bruno Clément, Kim-Anh Lê Cao, Orlando Musso

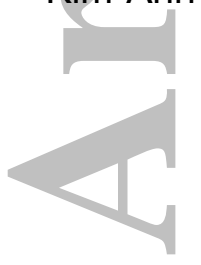

This file includes:

Page number: 38,

\author{
Table of contents,
}

Supporting Materials and Methods,

Supporting Figure Legends,

\section{Supporting Table Legends,}

References: 36 . 


\section{TABLE OF CONTENTS}

\section{Supporting Materials and Methods}

Tissue banking of the external validation in-house HCC collection 4

Statistical analysis $\quad 4$

Transcriptomic data processing 4

Development of a score predicting CTNNB1 mutational status 5

Prediction of CTNNB1 mutational status in nine external datasets $\quad 7$

Dataset quality control and combination for transcriptome meta-analysis 8

Discriminating robust HCC subclasses by hierarchical clustering 8

$\begin{array}{ll}\text { Functional genomics analyses } & 15\end{array}$

Periportal-type HCC gene signature $\quad 15$

$\beta$-catenin exon 3 sequencing and search for $\beta$-catenin exon 3 deletions $\quad 16$

Tissue microarray $\quad 17$

$\begin{array}{ll}\text { Primary antibodies } & 18\end{array}$

$\begin{array}{ll}\text { Cell culture } & 18\end{array}$

Nucleic acid extraction from human tissues and from in vitro cultures $\quad 18$

$\begin{array}{ll}\text { Real-time PCR } & 19\end{array}$

$\begin{array}{ll}\text { Sybr Green primers and TaqMan probes } & 20\end{array}$

RNA interference and cDNA vector transfection $\quad 20$

\section{Supporting Fig. legends}

Supporting Fig. $1 \quad 22$

Supporting Fig. $2 \quad 22$

Supporting Fig. $3 \quad 23$

Supporting Fig. $4 \quad 24$

Supporting Fig. $5 \quad 24$

Supporting Fig. $6 \quad 24$

Supporting Fig. $7 \quad 25$

$\begin{array}{ll}\text { Supporting Fig. } 8 & 25\end{array}$ 
$\begin{array}{lr}\text { Supporting Fig. } 9 & 26\end{array}$

$\begin{array}{ll}\text { Supporting Fig. } 10 & 27\end{array}$

Supporting Fig. $11 \quad 27$

Supporting Fig. $12 \quad 28$

Supporting Fig. $13 \quad 28$

$\begin{array}{ll}\text { Supporting Fig. } 14 & 28\end{array}$

Supporting Fig. 15

Supporting Tables

Supporting Table $1 \quad 30$

Supporting Table $2 \quad 30$

Supporting Table $3 \quad 31$

Supporting Table $4 \quad 31$

Supporting Table 5

Supporting Table $6 \quad 32$

Supporting Table $7 \quad 32$

Supporting Table $8 \quad 32$

Supporting Table 9

Supporting Table $10 \quad 32$

Supporting Table 11

Supporting Table 12

References to Supporting data $\quad 34$ 


\section{SUPPORTING MATERIALS AND METHODS}

\section{Tissue banking of the external validation in-house HCC collection}

The external validation in-house HCC collection (Fig. 1A) was recently described.(1) It included $70 \mathrm{HCC}$ patients undergoing liver surgery with curative intention at Rennes University Hospital between January 1992 and December 2007. Fifty-one were treated by partial hepatectomy and 19 by orthotopic liver transplantation. Anatomic pathology features of HCCs were reviewed and annotated by a senior pathologist (BT). Demographic, clinical and biological data were retrieved from hospital charts by two experienced liver surgeons (LS \& DB) and are described in online supporting table 2. Eighty-two frozen tumors from 70 patients were analyzed. As controls, we included 66 matching non-tumor livers and 5 histologically normal livers from patients undergoing resection of liver metastases of extrahepatic primary cancers. Non-tumor and normal liver samples were obtained at $>2 \mathrm{~cm}$ from the tumors. Frozen human tissue samples were processed by the Biological Resource Center, Rennes University Hospital (BB-0033-00056). Fresh tissues were frozen at $80^{\circ} \mathrm{C}$ in $\mathrm{N}_{2}$-cooled isopentane and stored at $-80^{\circ} \mathrm{C}$ under quality-controlled conditions. The study protocol complied with French laws and regulations and was approved by INSERM's Institutional Review Board (number 01-036) in the context of the National Network of Liver Biological Resource Centers. Sample collection was reported to the Ministry of Education and Research (No. DC-2008-338).

\section{Statistical analysis}

Transcriptomic data preprocessing 
Raw feature data from Gene Expression Omnibus were normalized and $\log _{2}$ intensity expression summary values for each probe set were calculated using Robust Multiarray Average (RMA, packages Affy or Limma). Each dataset was assessed for the detection of outliers and inter-array, experiment-related batch effects by principal component analysis (PCA) and density plots. The outliers removed were: one sample from GSE17856, one sample from GSE15765 and two samples from GSE54236. When clear-cut inter-array experiment-related batch effects were observed within a dataset, this effect was corrected by the COMBAT method (package sva)(2). GSE25097 was corrected for 2-batch effect and GSE14520 for 3-batch effect. Then, probes being detected over the background noise in at least one $\mathrm{HCC}$ were retained and quantile normalized (package preprocessCore). When raw data were not available, normalized data were directly used (GSE20140, GSE20140 and TCGALIHC-US).

$\gg$

Development of a score predicting CTNNB1 mutational status

To search for the best CTNNB1 mutation predictors and to assess their predictive power (Fig. 1A, C), we used three HCC sets annotated for Sanger-sequenced CTNNB1 exon 3 mutational status. The training set consisted of 87 HCCs (27 CTNNB1-mutant and 60 wild-type tumors) and the validation set contained 56 HCCs (17 CTNNB1-mutant and 39 wild-type tumors). CTNNB1 mutation markers were further validated in an 82-HCC in-house independent cohort (23 CTNNB1-mutant and 59 wild-type tumors) (Fig. 1A and supporting table 2). Training (GSE9843) and validation (E-TABM-36) sets were obtained from publically available HCC mRNA expression profiling datasets. Cross-platform data transformation was performed with YuGene to reduce the variance attributable to platform or experimental batch.(3) The 
external validation in-house $\mathrm{HCC}$ collection was analyzed by real-time PCR to validate the CTNNB1 mutation predictors in an independent population.

To search for predictors of CTNNB1 mutational status, we performed bootstrap over three PLS-DA principal components, whereby 300 rounds of bootstrap subsampling optimized the stability of predictors. Prediction was then validated on statistical tools based on one-tailed $t$-tests to objectively choose the number of optimal components and to identify the most robust set of predictors(4) (Supporting Fig. 2). The analysis identified Histidine Ammonia-Lyase (HAL) and Vanin 1 (VNN1) (selected in $>85 \%$ of the models after 300 bootstrap runs). Histidine ammonia-lyase (HAL) was downregulated in CTNNB1-mutant HCCs by 17 folds in the training set $[95 \% \mathrm{Cl}$ : (0.04-0.1), $\left.\mathrm{p}=2.8 \times 10^{-12}\right]$ and by 8 folds in the validation set $[95 \% \mathrm{Cl}:(0.06-0.23)$, $\left.\mathrm{p}=3.3 \times 10^{-6}\right]$. In turn, Vanin 1 (VNN1) was downregulated in CTNNB1-mutant HCCs by 16 folds in the training set [95\% Cl: $\left.(0.04-0.11), p=1.6 \times 10^{-12}\right]$ and by 7 folds in the validation set $\left[95 \% \mathrm{Cl}:(0.07-0.27), p=1.4 \times 10^{-5}\right]$ (Fig. $\left.2 \mathrm{~A}-\mathrm{B}\right)$.

Odontogenic Ameloblast-Associated Protein (ODAM) was identified by exploratory analysis searching for the highest fold change between HCCs carrying mutant versus wild-type CTNNB1. ODAM was upregulated by about 7 folds in the training set $[95 \%$ $\left.\mathrm{Cl}:(4-13), p=3.8 \times 10^{-6}\right]$ and by 10 folds in the validation set $\left[95 \% \mathrm{Cl}:(4-24), \mathrm{p}=2.8 \times 10^{-}\right.$ 5].

To develop a robust scoring method of prediction, we also included two well-known CTNNB1 mutation markers, namely GLUL and LGR5.(5-8) GLUL was upregulated by about 2 folds in the training set [95\% Cl: $\left.(2-3) ; p=6.4 \times 10^{-12}\right]$ and by 6 folds in the validation set [95\% Cl: (4-10); $\left.\mathrm{p}=7.7 \times 10^{-7}\right]$; $L G R 5$ was upregulated by about 7 folds in the training set $\left[\mathrm{Cl}:(4-11) ; p=6.3 \times 10^{-8}\right]$ and by 9 folds in the validation set $[95 \% \mathrm{Cl}$ : $\left.(5-17) ; p=8.3 \times 10^{-7}\right]$. After further validation of these markers in the external validation 
in-house HCC collection by real-time PCR (Fig. 2A,B), we built an arithmetic score as shown in Fig. 2C.

\section{Prediction of CTNNB1 mutational status in nine datasets}

After extraction of gene expression values for GLUL, LGR5, ODAM, HAL and VNN1 from the training and validation sets and from the nine datasets used for transcriptome meta-analysis, we compared:

1) Three normalization techniques (Autoscaling, Median Centering and TotalSum Normalization).(9)

2) Several combinations of the five CTNNB1 mutation markers: alternative scores with $<5$ genes were compared with the 5-gene score.

For each normalization technique and gene combination, the predictive score was calculated and the common threshold maximizing sensitivity and specificity in both training and validation sets was evaluated. Then, in each of the nine datasets, samples with a score higher than the threshold were predicted as mutated. To find the best method, we evaluated the performance of the score in the training and validation sets and the predicted mutation rate in each of the nine datasets, in comparison with the expected mutation rates in HCCs $(20 \%$ to $40 \%) .(5-7,10,11)$ This optimization process led us to choose the median centering technique and the 5-gene score. The performance of this method and the predicted mutation rate in the nine datasets is presented in supporting table 5.

Dataset quality control and combination for transcriptome meta-analysis 
To assess the quality of each dataset, Pearson correlation coefficients were calculated for gene sets expected to be correlated: KRT19-EPCAM,(12) GLULLGR5(7) and ODAM-HAL-VNN1 (supporting table 3). Datasets were discarded from the meta-classification analysis if Pearson correlations were $<0.25$ for at least one of the gene couples tested. Thus, nine datasets passing the quality control analysis were combined into a transcriptomic metadata set intended for meta-classification (Fig. 1B and supporting table 2), that totalized 1133 HCCs. Genes included in this metadata set were matched by ENTREZ ID (package org.Hs.eg.db). When multiple probe sets mapped a unique gene, we used the probe with a $p$-value detection $<0.05$

in most samples. The nine datasets had 9542 common genes. Probes were merged to the gene level and all nine datasets were normalized with the COMBAT algorithm to eliminate platform-related batch effect(2) (Supporting Fig. 5). Thus, the metadata set consisted of 1133 HCCs with 9542 common genes.

\section{Discriminating robust HCC subclasses by hierarchical cluster analysis}

Hierarchical cluster analysis on the metadata set was performed with the Ward's method using 1-Pearson correlation as a distance metric. To highlight robust HCC subclasses, we applied the following four steps which are outlined below.

1) Identifying HCC samples highly associated with a subclass by individual reproducibility analysis.

2) Isolating the most highly associated gene sets within each $\mathrm{HCC}$ cluster by PLS-DA modeling.

3) Refining the HCC classification with the most highly associated gene sets within each $\mathrm{HCC}$ cluster. 
4) Evaluating the robustness of the generated subclasses by several techniques, namely global reproducibility analysis, PCA, measure of intercluster and intra-cluster inertia and PLS-DA modeling.

Detailed description of the four steps applied to highlight robust HCC subclasses

1) Identifying HCC samples highly associated with a subclass by individual reproducibility analysis.

To isolate HCCs robustly linked to each cluster, we calculated an individual reproducibility score applying the following steps, modified from Boyault et al.,(13):

A. Unsupervised gene selection based on the two following criteria:

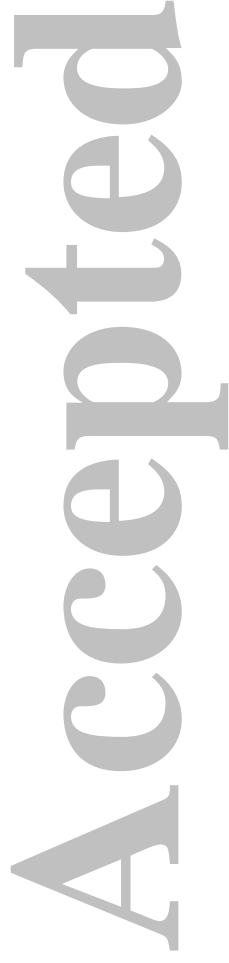

i. Variance test $p<0.01$. For each gene $(G)$ we tested whether its variance across samples was different from the median of the variances of the 9542 genes. The statistic used was $[(n-$ 1) $\left.\times \operatorname{Var}(G) / \operatorname{Var}_{\text {med }}\right]$, where $n$ refers to the number of samples. This statistic was compared to a percentile of the Chi-square distribution with (n-1) degrees of freedom and yielded a p-value for each gene; and

ii. "robust" coefficient of variation (rCV) less than 10 and superior to a given $\mathrm{rCV}$ percentile threshold. Six rCV percentile thresholds were used: 60\%; $70 \% ; 80 \% ; 90 \% ; 95 \% ; 97 \%$, yielding six gene lists. The rCV for each gene was calculated by dividing 
the standard deviation by the mean. It was called "robust" because the highest and the lowest expression values across the samples for each gene were deleted.

B. Hierarchical clustering of the 1133 samples using the whole metadata set or data restricted to each of the six gene sets defined by the rCV percentile thresholds (see ii above) produced seven dendrograms. As the "elbow" method in the whole metadata set suggested a 4-cluster classification (not shown), the seven dendrograms were cut into four clusters.

C. Individual reproducibility score: For each dendrogram, a sample was noted 1 for a given cluster (1-4) if it was classified in this cluster and 0 if it was not. The individual reproducibility score of clusters 1-4 was calculated with the mean of the scores of the seven dendrograms. Then, samples with a reproducibility score over 0.85 for a given cluster (which means that an HCC was classified in this cluster in at least six dendrograms out of seven) were considered to belong to this cluster in a robust way. Thus, 158 samples ( $14 \%$ of the metadata set) were robustly associated with cluster 1 (red); 123 (11\% of the metadata set) were robustly associated with cluster 2 (purple); 228 (20\% of the metadata set) were robustly associated with cluster 3 (green) and none of the samples were robustly associated with cluster 4 (blue).

\section{Hepatology}

This article is protected by copyright. All rights reserved. 
2) Isolating the most highly associated gene sets within each HCC cluster by PLS-DA modeling

Cluster-specific gene sets were determined by Partial Least Squares Discriminant Analysis (PLS-DA, package pls), following these steps:

A. Gene sets that were significantly different by a Tumor versus non-tumor $t$ test, showing a fold difference either $<0.5$ or $>2.0$, were considered to be tumor specific. For this test, the dataset GSE14520 was used and 2881 genes were selected.

B. Three PLS-DA models were obtained on the metadata set with 2881 differentially expressed genes for the prediction of the three subclasses defined by individual reproducibility analysis (red, purple and green). The best number of principal components $k$ were evaluated after a cross-validation by the PRESS criteria $(k(r e d)=8$, $\mathrm{k}($ purple $)=5$ and $\mathrm{k}($ green $)=7)$. Then, variable importance in the projection (VIP) was calculated for each gene and each principal component. To refine the HCC classification, the mean of $k$ VIPs in at least one PLS-DA model was set to >1, yielding 1618 genes. For gene ontology analysis, the mean of $k$ VIPs in at least one PLS-DA model was set to $>1.5$, yielding 550 genes.

3) Refining the HCC classification with the most highly associated gene sets within each HCC cluster 
The HCC classification was refined by a new hierarchical cluster analysis on the metadata set using the 1618 gene set (VIP>1). The dendrogram was cut into four clusters, as suggested by the "elbow" method (Supporting Fig. 8).

4) Evaluating the robustness of the generated subclasses from the 1618 gene set $(\mathrm{VIP}>1)$ by global reproducibility analysis, $\mathrm{PCA}$, measure of inter-cluster and intra-cluster inertia and PLS-DA modeling.

A. Global reproducibility analysis included the following steps, modified from Boyault et al.,(13):

i. Unsupervised gene selection was based on the two following criteria :

a. Variance test with a p-value $<0.01$

b. Robust coefficient of variation (rCV) less than 10 and superior to a given rCV percentile from the following threshold list: $60 \% ; 70 \% ; 80 \% ; 90 \%$; 95\%; 97\%, 99\%, 99.5\%, yielding eight gene lists.

ii. Hierarchical clustering of the 1133 samples using the entire metadata set or data restricted to each of eight gene set lists produced nine dendrograms. Each dendrogram was cut into four clusters.

iii. For each dendrogram, the global reproducibility score was assessed by calculating the \% of samples well classified. 
iv. The same score was calculated for each dendrogram restraining the data to a subclass of samples (red, purple, green and blue). These analyses showed that the red, green and purple subclasses were robust, with a global reproducibility higher than $70 \%$ in most of the cases. By contrast, the blue subclass was less robust, consistently with individual reproducibility analysis. The results of these analyses are presented in Supporting Fig. 6.

B. PCA analysis was performed with the FactoMineR package. Individual score plot in the first two principal components according to individual classification show noticeable discrimination (Supporting Fig. 7A).

C. The intra-cluster and the inter-cluster inertia were measured by Euclidean distances. The most homogenous cluster (with the weakest intra-cluster inertia) was the green one $(I=55.76)$, then the red one $(I=58.18)$, the blue one $(I=59.77)$ and the purple one (I=69.14). The mean intra-cluster inertia, that can reflect dispersion, was weaker than the mean inter-cluster inertia, than can reflect distance between clusters. The green cluster was closest to the red cluster and both green and red clusters were distant from the purple one, the blue cluster occupying an intermediate position between them (Supporting Fig. 7B). 
D. Multivariate PLS-DA analysis (package pls) was performed to measure the association between gene expression and the four subclasses. The model was validated by a 10-9 double crossvalidation and the significance by a 1000-permutation test, as described.(14) The model was able to significantly discriminate the four subclasses, with a double cross-validated correct classification rate of $89 \%$ (SD: 0.03$),\left(p=10^{-3}\right)$. In addition, the score plots in the first two or three principal components (2D-3D plot) according to the individual classification showed noticeable discrimination of the clusters. It also illustrated the distance between them, with intermediate position of the blue cluster as described in the inertia analysis (Supporting Fig. 7C-D). 


\section{Functional genomics analyses}

Gene annotations based on gene ontology and enrichment for biological functions were done with the FuncAssociate 2.1 program.(15) Gene set enrichment analysis (GSEA) was performed with the web-based tool developed at the Broad Institute.(16)

\section{Periportal-type HCC gene signature}

After functional genomics analysis, the green cluster was defined as representative of a HCC subclass associated with periportal metabolism, thus called Periportal-type. Then, we searched the 9542 genes within the 1133 HCC metadata set for top Periportal HCC genes as good outcome predictors. Selected genes complied with the following four stringent criteria:

1) Fold change $>2$ between Periportal-type samples and the rest of the samples in the 1133 HCCs metadata set.

2) Periportal gene, as defined by the periportal signature of normal mouse liver.(17)

3) Hub gene in the network of metabolic liver zonation: connectivity (i.e., Pearson's correlation coefficient $>0.30$ ) with $>50 \%$ of 226 periportal or perivenous genes, as assessed by network analysis. Periportal and perivenous genes were defined according to normal mouse liver periportal and perivenous signatures.(17)

4) Association with overall and disease-free survival analyses in 183 HCCs predicted as carrying wild-type CTNNB1 from dataset $C$ ( $n=247$ patients).(18) To this end, a gene was retained when at least 7 out of the 8 univariate survival analyses detailed below were statistically significant $(p<0.05)$ : 
a. Two Cox analyses (overall and disease-free survival analyses);

b. Six log-rank analyses using three different gene-expression-level cutoffs: percentile 25, median and percentile 75 (overall and diseasefree survival analyses).

Only eight genes complied with the above criteria and were retained to define the Periportal signature. The $247-\mathrm{HCC}$ patients in dataset C and the $210-\mathrm{HCC}$ patients in the external validation RNAseq cohort were then studied by PCA analysis of the 8gene signature. HCCs with a score >median in the first principal component were defined as Periportal HCCs.

\section{CTNNB1 exon 3 sequencing and search for CTNNB1 exon 3 deletions}

CTNNB1 exon 3 sequencing was performed as described.(19) For mutation analysis, exon 3 and the flanking intronic sequences of the CTNNB1 gene were amplified by PCR using Kappa HiFi DNA polymerase (Kapa Biosystems, Wilmington, MA). Briefly, $50 \mathrm{ng}$ of genomic DNA were used as template with $\beta$ CATEX2F (5'-GAA-AAT-CCAGCG-TGG-ACA-ATG-3') and BCATEX4R (5'-TCG-AGT-CAT-TGC-ATA-CTG-TCC$\left.3^{\prime}\right)$ as oligonucleotide primers. The reaction included a $95^{\circ} \mathrm{C}$ denaturation step for 3 min followed by 35 cycles consisting of: $98^{\circ} \mathrm{C}$ denaturation for $15 \mathrm{sec}, 60^{\circ} \mathrm{C}$ annealing for $15 \mathrm{sec}$, and elongation $72^{\circ} \mathrm{C}$ for $30 \mathrm{sec}$. Both strands of the amplified products were directly sequenced on an automated sequencer (ABI 3700; PerkinElmer, Boston, MA). Negative results on exon 3 mutations may overlook large exon 3 deletions in CTNNB1. Indeed, as somatic deletions are heterozygous, the wild-type allele may mask the deletion upon Sanger sequencing.[40] To avoid this pitfall, HCCs showing no exon 3 mutation were screened for $\beta$-catenin deletion by PCR on 
reverse-transcribed tumor cDNA. For PCR amplification of the exon 3 of CTNNB1, oligonucleotide primers (F1: 5'-GCG-TGG-ACA-ATG-GCT-ACT-CAA-G-3'; R1: 5'TAT-TAA-CCA-CCA-CCT-GGT-CCT-C-3'; and R2: 5'-TTC-AGC-ACT-CTG-CTTGTG-GTC-3') were used. The primer pairs F1-R1 and F1-R2 yielded 517 bp (169 aa) and 1043 bp (344 aa) amplicons, respectively. As positive control, HepG2-derived cDNA was used, as these cells harbor a large CTNNB1 deletion (amino-acids 25140).[40] PCR was performed using the GoTaq Green Master Mix (Promega) and amplicons migrated in $1.5 \%$ Nusieve agarose (LifeTechnologies) gels.

\section{Tissue microarray}

Tissue microarrays were set up and analyzed as previously described.(19, 20) For selection of tissue blocs for TMA construction, the whole set of archival HE-stained sections from formalin-fixed, paraffin-embedded tissue blocks from each one of the 67 available HCC patients and from five histologically normal livers were reviewed (Nikon 80i microscope) to ensure that the selected paraffin bloc was the mirror lesion of the frozen sample. A region of interest (ROI, 0.5 to $1 \mathrm{~cm}$ in diameter) was labeled with a permanent marker to precisely localize the punch zone. TMA construction was done with a MiniCore3 tissue arrayer (Alphelys, Plaisir, France). ROls were punched in triplicate. Punch size was set at $1000 \mu \mathrm{m}$ in diameter. HCC samples were randomly assigned to either one of two 132-punch receiver paraffin blocks. Randomization was performed with a "rand" function in Microsoft Excel. The five histologically normal liver controls were included in both array blocs \#1 and \#2 to control for signal and background intensities. Five- $\mu m$ microtome sections were processed for immunohistochemistry with a Discovery XT from Ventana Medical Systems (Roche) slide staining system. See the table below for antibodies. Stained 
slides were converted into high-resolution digital data with a NanoZoomer digital slide scanner (Hamamatsu, Massy, France). Digital slides were viewed using NDP.view software (Hamamatsu) in a Dell S2415H 24-Inch Screen LED-Lit Monitor.

\section{Primary antibodies}

\begin{tabular}{|c|l|l|}
\hline \multicolumn{3}{|c|}{ PRIMARY ANTIBODIES } \\
\hline GENE ID & CAT. \# & Supplier \\
\hline$\beta$-catenin & 610154 & BD Biosciences \\
\hline GLUL & 610518 & BD Biosciences \\
\hline HAL & HPA038547 & Sigma Aldrich \\
\hline ODAM & $16509-1-A P$ & Proteintech \\
\hline VNN1 & sc-16776 & $\begin{array}{l}\text { Santa Cruz } \\
\text { Biotechnology }\end{array}$ \\
\hline
\end{tabular}

\section{Cell culture}

297T, BC2, B16, Hep-G2, Huh-6 and Huh-7 cells were cultured in DMEM.(21) HepaRG cells were cultured as previously described.(1, 22, 23) For BIO assay, cells were treated with BIO (Merck Millipore) $2.5 \mu \mathrm{M}$ during $72 \mathrm{~h}$, applying a previously optimized protocol.(24)

\section{Nucleic acid extraction from human tissues and from in vitro cultures}

RNA and DNA were extracted from $10 \mu \mathrm{m}$ cryosections obtained from frozen tissue blocks, as previously described(19, 20). Once tissues were placed on the specimen head of a Leica 3050 s cryostat and trimmed to obtain a level and histologically controlled representative surface (hematoxylin or toluidine blue extemporaneous staining), the first two $5 \mu \mathrm{m}$ sections were set aside as permanent histological 
controls. Then, $10 \mu \mathrm{m}$ cryosections were obtained and immediately lysed in RNA or DNA extraction buffers. Before removing the disk containing the specimen from the head, two $5 \mu \mathrm{m}$ cryosections were set aside again as permanent histological controls. Lead and rear permanent histological controls were formalin-fixed, HEstained in a Leica ST5020 routine stainer and representativity was assessed by two independent observers (BT \& OM). Non-representative samples were removed from the RNA and DNA extraction process. Representative samples were extracted with NucleoSpin® RNA II (Macherey-Nagel) and with the DNeasy Blood \& Tissue (Qiagen) kits, as recommended by the manufacturers. Quality control of the extracted

nucleic acids was performed by dosing the eluents with a NanoDrop spectrophotometer and by checking $28 \mathrm{~S} / 18 \mathrm{~S}$ ratios by gel electrophoresis. For in vitro cultured cell layers, total RNA was purified with an RNAeasy kit (Qiagen). Extracted RNA quality control was done as for human tissues.

\section{Real-time PCR}

Real-time PCR was performed as described.(19, 20) Reverse-transcribed cDNA was obtained with the High Capacity cDNA Reverse Transcription Kit (Applied Biosystems) according to the manufacturer's instructions. Expression of relevant genes was measured by quantitative real-time PCR using the StepOnePlus ${ }^{\mathrm{TM}}$ System or the ABI prism 7900HT, power SYBR Green PCR master mix or Taqman probe-based assays (Applied Biosystems), according to the experiments. Quantitative analyses of PCR data were conducted by the $2^{-\Delta \Delta C t}$ method. See below for Sybr Green and TaqMan probes used. 
Sybr Green primers and TaqMan probes

\begin{tabular}{|c|c|c|}
\hline \multicolumn{3}{|c|}{$\begin{array}{c}\text { SYBR GREEN PRIMERS } \\
\text { (Eurogentec, Seraing, Belgium) }\end{array}$} \\
\hline GENE ID & STRAND & Primer sequences 5'- 3' \\
\hline \multirow{2}{*}{$A L D O B$} & Forward & GCATCTGTCAGCAGAATGGA \\
\hline & Reverse & TAGACAGCAGCCAGGACCTT \\
\hline \multirow{2}{*}{ AXIN2 } & Forward & TCAGCAGAGGGACAGGAATC \\
\hline & Reverse & TCTTTGGCTCTTTGTGATCTTCTG \\
\hline \multirow{2}{*}{ GLUL } & Forward & AAGTGTGTGGAAGAGTTGCC \\
\hline & Reverse & TGCTCACCATGTCCATTATC \\
\hline \multirow{2}{*}{$H A L$} & Forward & CAACAAGCTCTCCACCAGTT \\
\hline & Reverse & CTCCTTGTTGGTTTTAATCACCTC \\
\hline \multirow{2}{*}{ HNF4A } & Forward & ACATGGACATGGCCGACTAC \\
\hline & Reverse & TCTGATGGGGACGTGTCATTG \\
\hline \multirow{2}{*}{ HPRT1 } & Forward & TGACACTGGCAAAACAATGCA \\
\hline & Reverse & GGTCCTTTTCACCAGCAAGCT \\
\hline \multirow{2}{*}{ LGR5 } & Forward & GAAGCTCTGCAGAATTTGCGAAGCC \\
\hline & Reverse & CAGGTGCCTCAGGGAATGCAGG \\
\hline \multirow{2}{*}{ TBP } & Forward & GAGCTGTGATGTGAAGTTTCC \\
\hline & Reverse & TCTGGGTTTGATCATTCTGTAG \\
\hline \multirow{2}{*}{ VNN1 } & Forward & GCACGCTACCATAAGCAAAAC \\
\hline & Reverse & ACAATCTCAGGCTCCTTGGG \\
\hline
\end{tabular}

\begin{tabular}{|c|l|}
\hline \multicolumn{2}{|c|}{ TAQMAN PRIMERS \& PROBE } \\
\hline $\begin{array}{c}\text { GENE } \\
\text { ID }\end{array}$ & Life Technologies cat. \# \\
\hline ODAM & Hs002152928m1 \\
\hline
\end{tabular}

RNA interference and cDNA vector transfection

Routinely expanded HepaRG progenitor cells were detached by trypsinization, seeded at low density $\left(2 \times 10^{4}\right.$ cells $\left./ \mathrm{cm}^{2}\right)$, detached three days later and transfected in suspension with small interfering RNAs (siRNAs) by a MP100 Microporator (LabTech) and Neon ${ }^{\mathrm{TM}}$ Transfection System $100 \mu \mathrm{L}$ Kit (Invitrogen) under optimized conditions ( 80 pmoles siRNA, $1500 \mathrm{~V}, 20 \mathrm{~ms}, 1$ pulse), as we previously reported.(19) 
Silencer negative control (D-001810-10-05, Thermo Fisher) and $\beta$-catenin (HSS102460, Invitrogen) siRNAs were used.
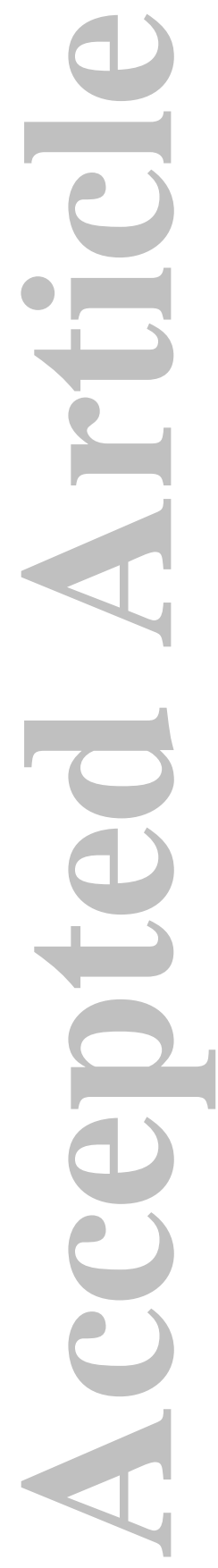

Hepatology

This article is protected by copyright. All rights reserved. 


\section{SUPPORTING FIGURE LEGENDS}

Supporting Fig. 1. Quality analysis of the external validation cohort (TCGA-LIHCUS). (A) Patient selection. (B) Assessment of the quality of extraction of clinical annotations by Kaplan-Meier plots of overall and disease-free survival as a function of TNM staging.

Supporting Fig. 2. Choosing predictive and stable gene signatures by sPLS-DA (A) Bootstrap subsampling evaluation of the stability of each gene predictor across three SPLS-DA components, where frequency of selection over 300 bootstrap subsamplings (Y-axis) is shown per gene (ENSEMBL ID, X-axis). Labels are provided for the 3 genes contributing to the signature across one component. Component 1 (blue rectangles), Component 2 (orange triangles), Component 3 (grey inverted triangles).

(B) Evaluation of the contribution of each gene set to the informativeness of the first component. Each dot is a gene set ordered along the X-axis by decreasing stability (frequency of selection). The $\mathrm{Y}$-axis represents the $-\log _{10}(P$-value $)$ of a one tailed $t$ test indicating improvement in classification accuracy. (C) Classification accuracy of the 3-gene signature on both training and validation sets.

\section{Comments to Supporting Fig. 2.}

One PLS-DA component allowed the identification of three markers associated with CTNNB1 mutations in HCCs, namely HAL, VNN1 and CCDC170. Together, these three markers accurately classified more than $87 \%$ of the samples in both the training and the validation sets (see C). However, because of the low frequency of selection of $C C D C 170$ (see A), the analysis focused on both HAL and VNN1 (frequency of selection $>85 \%$, see $A$ ). 
Supporting Fig. 3. Predictive accuracy of the 5-gene score applied to tumor/nontumor gene expression ratios on CTNNB1 mutational status in the external validation HCC collection analyzed by qPCR. (A) Correlations between GLUL, LGR5, ODAM, HAL and VNN1 in the training and validation sets and in the external validation HCC collection analyzed in HCCs (T); non-tumor samples (NT) and in tumor/non-tumor ratios. AXIN2 is used as an indicator of high $\beta$-catenin pathway signaling.(25) (B) Heatmap showing the correlations of nuclear $\beta$-catenin $\left(N u \_\beta\right.$-cat) with the mRNA expression of the five biomarkers and CTNNB1 mutational status (Mut_ß-cat), assessed by immunohistochemistry scoring as described,(1) in 82 HCCs. Each blue cell in the top row represents one HCC. Low and high gene expressions (using the median as the cut-off value) are represented in green and red, respectively. $\gamma$, Gamma correlation coefficient. (C) $\log _{2}$ of the Tumor/non-tumor ratios of mRNA expression levels for the indicated genes, according to CTNNB1 mutational status. (D) Performance of the combined score of tumor/non-tumor gene expression ratios of GLUL, LGR5, ODAM, HAL and VNN1 to predict CTNNB1 mutated HCCs. The combined score was calculated as follows, using tumor/non-tumor ratios: Score $=$ (GLUL $\times$ LGR5 $\times O D A M) \div(H A L \times V N N 1)$. (E) Receiver operating curve (ROC) showing predictive performance of the combined score on CTNNB1 mutation. (F) Relative gene expression of the biomarkers in HCCs (blue) and non-tumor (red) samples according to CTNNB1 mutational status ("mutated" and "wild-type" HCCs). ${ }^{*} \mathrm{P}<0.05,{ }^{* *} \mathrm{P}<0.01,{ }^{* *} \mathrm{P}<0.001$, after Wilcoxon tests. $\mathrm{Cl}$, Confidence interval; Se, Sensitivity; Sp, Specificity. AUROC, area under the ROC curve. 
Supporting Fig. 4. Real-time PCR-based gene expression of $H A L, V N N 1$ and ODAM in HCC cell lines carrying mutant (B16, BC2, HepG2, Huh6) or wild-type (Huh7, HepaRG) CTNNB1,(10, 22) measured by real-time PCR. Results are expressed as relative to the 293T cell line (WT CTNNB1). Data are displayed as mean values $\pm S D$. Experiments were done in triplicate.

Supporting Fig. 5. Functional analyses of the five mutation biomarkers by real-time PCR in HepaRG cells. (A) The GSK3 $\beta$ inhibitor BIO $(2.5 \mu \mathrm{M})$ strongly upregulates $\beta$ catenin transcriptional activity, as shown in a $\beta$-catenin gene reporter assay, where 8xtandem TCF sites control the expression of the Firefly luciferase gene. (B) BIO upregulates GLUL, LGR5 and ODAM mRNA expression, assessed by real-time PCR. (C-F) BIO attenuates the gain in the expression of HNF4A, ALDOB, HAL and VNN1 mRNA upon differentiation of HepaRG liver progenitors into hepatocyte-like cells over

a 30-day time course. (G) HepaRG cells transfected with $\beta$-catenin targeting siRNA downregulate $A X I N 2$ and $O D A M$ and upregulate $H A L$ and VNN1 mRNA expression (real-time PCR). Data are relative to scrambled siRNA-transfected cells. Mean \pm SD. Experiments were done in triplicate.

Supporting Fig. 6. Combat performance. The COMBAT algorithm effectively removes cross-platform variance effects after merging nine transcriptomic datasets into a metadata set. (A) Score plot of the individuals in the first two principal components of a principal component analysis (PCA). The 9542 common genes in the nine datasets are shown after preprocessing. PCA reveals cross-platformdependent correlations. (B) Score plot of the individuals in the first two principal 
components of PCA after removal of cross-platform variance using the COMBAT algorithm.(26)

Supporting Fig. 7. Unsupervised hierarchical cluster analysis reveals strong enrichment of HCCs with predicted CTNNB1 mutations in one HCC subclass. (A) The dendrogram was obtained with Ward's linkage and 1-Pearson correlation coefficient on the basis of the expression profile of 1618 genes, which were the most discriminant of the four subclasses (VIP>1 after PLS-DA analysis) in the metadata HCC set. Top, the four HCC subclasses (purple, blue, red and green nodes) are shown, as well as the predicted CTNNB1 mutational status of each tumor (red: mutant; blue: wild-type). The $\%$ of predicted CTNNB1 mutations in each HCC subclass is indicated in boxes. Left, for each gene, the lowest and highest intensity values are represented in green and red, respectively.

Supporting Fig. 8. Reproducibility analysis performed on the four HCC subclasses. The numbers of HCCs in the whole metadata set and in each one of the subclasses are as follows: whole metadata set $(n=1133)$, black curve; subclasses: green $(n=326) ;$ red $(n=210) ;$ blue $(n=420) ;$ purple $(n=177)$. The $\%$ of well-classified samples after hierarchical clustering using Ward's linkage and 1-Pearson correlation coefficient is shown as a function of gene sets containing different proportions of the most variable genes included in each analysis.

Comments to Supporting Fig. 8. The robustness of the four subclasses was measured by a global reproducibility score plotting the \% of well-classified samples as a function of the gene sets containing variable percentages of the most variable 
gene subsets. Thus, this analysis was performed on $100 \%$ of the genes (i.e., 9542gene set) in the metadata set or on gene sets containing the most variable proportions of genes according to different thresholds $(40 \%, 30 \%, 20 \%, 10 \%, 5 \%$, $3 \%, 1 \%, 0.5 \%)$. The red, green and purple subclasses are homogenous, with a global reproducibility higher than $70 \%$ at different thresholds. By contrast, the blue subclass is less homogenous, consistently with individual reproducibility analysis.

Supporting Fig. 9. (A-D) Robustness analysis and distance measures between the four subclasses in the metadata set: (A) Principal component analysis (PCA) showing the individual score plot according to HCC subclasses. The four subclasses are noticeably discriminated. (B) Intra-cluster and inter-cluster inertia. Mean intra-cluster inertia (orange cells), that can reflect dispersion, is weaker than the mean of intercluster inertia (yellow cells), than can reflect distance between subclasses. (C) 3D representation of the score plot of multivariate PLS-DA in the first three principal components. The model is able to significantly discriminate the four subclasses, with $89 \%$ of samples correctly classified after a $10-9$ double cross-validation (SD: $0.03, p$ value $=10^{-3}$ after 1000 permutations). (D) Comparison of the 4-clusters obtained by two discriminant analysis cut-offs: (i) 1618 genes (VIP>1); and (ii) 550 genes (VIP>1.5 after PLS-DA analysis, see supporting materials and methods). Using either of the two cut-offs (VIP>1 \& VIP>1.5), $81.6 \%$ of the samples fall within the same cluster. (E) Validation of the top discriminant 550 genes as HCC classifiers by unsupervised hierarchical clustering in the external validation cohort (210-HCC RNAseq dataset; TCGA, LIHC, US). HCC subclasses are indicated in color. For each gene, lowest and highest intensity values are represented in green and red, respectively. 
Supporting Fig. 10. TP53 gene expression and TP53 mutation biomarkers in the transcriptomic subclasses from the HCC metadata set. Results are expressed as relative to the Periportal-type subclass. (A) Three markers of TP53 mutation in cancer $(27,28)$ are overexpressed in the STEM-type subclass. (B) TP53 gene expression in the individual datasets composing the metadata set. As TP53 was missing in dataset $D$, its expression was explored separately in each of the remaining datasets composing the metadata set. TP53 was significantly upregulated in STEMtype HCCs in some of the datasets and particularly in the dataset with the largest number of samples (Dataset C, $n=247)$. Green, PP ( $n=326)$; Red, PV ( $n=210)$; Blue, ECM ( $n=420)$; Purple, STEM $(n=177) . \quad N S$, non-significant. ${ }^{*} \mathrm{P}<0.05,{ }^{* *} \mathrm{P}<0.01$, ${ }^{* * *} P<0.001$ after Student's $t$ tests.

Supporting Fig. 11. Expression of hallmark genes representing the HCC subclasses. Gene expression results are shown as relative to Periportal-type HCCs. Green, PP (Periportal-type, $n=326$ ); red, PV (Perivenous-type, $n=210$ ); blue, ECMtype (extracellular matrix, $n=420$ ); purple, STEM-type (stem cell type, $n=177$ ). (A) Wnt pathway and perivenous zonation genes upregulated in the PV group. (B) Examples of genes expressed at higher levels in Periportal-type HCCs. (C) Tumor stem/progenitor cell (AFP, EPCAM, PROM1, KRT19, SOX9, CD44, DKK1) and extracellular matrix remodeling (TGFB1, VCAN, TNC, LAMC1, VIM) genes are overexpressed in STEM-type and ECM-type HCCs, respectively. NS, non-significant. ${ }^{*} \mathrm{P}<0.05,{ }^{* *} \mathrm{P}<0.01,{ }^{* * *} \mathrm{P}<0.001$ after Student's $t$ tests. 
Supporting Fig. 12. Mouse liver zonation and HNF4A target genes discriminate human HCC subclasses. (A) Unsupervised hierarchical clustering of 210 Perivenoustype and 326 Periportal-type HCCs based on 93 periportal $(P P)$ and 79 perivenous (PV) mouse ortholog genes.(17) Green indicates lower expression; red higher expression. Periportal-type and Perivenous-type HCC subclasses are remarkably discriminated. (B) Unsupervised hierarchical clustering of 1133 human HCCs based on the expression profile of the genes most tightly modulated by HNF4A in Hnf4a-KO mice(29) (fold change $>2$ or $<0.5$; Bonferroni adjusted $P$ value $<0.01$ ). Over the 1868 genes identified in Hfn4a-KO mice, 603 orthologs were available in the human 1133HCC metadata set. On the left panel, relative gene expression between Hnf4a-KO and Hnf4a-WT is indicated in color (Green: WT; Red: KO). On the top panel, the four HCC subclasses are indicated with the color keys under "Tumor (subclass)". Hierarchical clustering discriminates the four subclasses, with $80 \%$ of samples correctly classified. Periportal-type HCCs group are strongly enriched in genes upregulated in Hnf4a-WT mice.

Supporting Fig. 13. Kaplan-Meier plot of overall survival of individuals in the external validation cohort (210-HCC RNAseq dataset; TCGA, LIHC, US) according to HCC subclass. HCC subclasses are indicated in color. Green, Periportal-type; Red, Perivenous-type; Blue, ECM-type; Purple, STEM-type HCCs. ${ }^{*} \mathrm{P}<0.05,{ }^{* *} \mathrm{P}<0.01$. after log-rank tests.

Supporting Fig. 14. The periportal signature is composed of liver-enriched genes.
(A) $A G X T$
(B) FETUB, (C) GLS2,
(D) GNMT,
(E) OTC
(F) SLC10A1, (G) SLC22A7 
and $(H)$ SLC27A5 mRNA expression data were extracted from the GSE1133 microarray dataset consisting of a panel of 158 samples including 84 different human tissues. Normalized background-corrected intensities of mRNA expression(30) were filtered to reveal the tissues expressing the highest ( $>3 x$ median, $>10 x m e d i a n$ or $>30 x m e d i a n)$ mRNA levels for each gene. Histograms show mean \pm SEM for each tissue type. AGXT, FETUB, GLS2, SLC10A1, SLC22A7 and SLC27A5 are expressed over the threshold of $30 x$ median only in liver samples. GNMT is expressed over this threshold in liver and pancreatic islets. OTC is expressed over the threshold of $3 x$ median in liver, small intestine, fetal liver and ganglion samples.

Supporting Fig. 15. Expression of the components of the Periportal HCC Signature in non-tumor livers (NT), Periportal-type HCCs (PP) and the other HCC subclasses (other HCCs). "Other HCCs" include: Perivenous-type, ECM-type and STEM-type. Samples were extracted from the GSE14520 microarray dataset. Each individual dot corresponds to a tissue sample. The statistical differences between means (horizontal bars) were calculated by ANOVA. 


\section{SUPPORTING TABLES}

Supporting Table 1. Bibliographic review of studies including HCC patients carrying sequence-verified exon 3 CTNNB1 ( $\beta$-catenin) mutations versus HCC patients carrying sequence-verified wild-type CTNNB1 and performing survival analyses: CTNNB1 exon 3 mutations were reported to be associated with survival in one cohort.(31) However, in this cohort,(31) patients with HCCs carrying wild-type CTNNB1 were more frequently HBV-infected: [(HBV in liver tissues: mutant CTNNB1, 9\%; wild-type CTNNB1, $24 \%\left(\mathrm{p}<10^{-6}\right)$; HBsAg in serum: mutant CTNNB1, $46.8 \%$; wild-type CTNNB1, 71.5\% $\left.\left(\mathrm{p}=1.3 \times 10^{-3}\right)\right] .(31)$ Thus, HBV infection may constitute a confounding factor because HCCs carrying wild-type CTNNB1 and arising in a background of HBV infection tend to meet the criteria for high-proliferation class HCCs with bad outcome.(11) In other publications, the association between CTNNB1 mutation and overall, disease-free survival or disease-specific survival was not significant, even in large cohorts (two large cohort studies with 307(32) and 362(33) annotated patients, respectively). In three publications, CTNNB1 exon 3 sequencing and patient survival data were available, but no survival analysis comparing HCCs carrying mutated CTNNB1 versus those carrying wild-type CTNNB1 were reported.(34-36) NA, information Not Available (gray cells); NS, non-significant (light blue cells); statistically significant ( $p<0.05$, green cells); HR, Hazards ratio (Cox's Hazards regression); OR, Odds ratio (logistic regression).

Supporting Table 2. Clinical features of HCCs from public datasets and external validation in-house HCC collection used for biomarker search and transcriptome meta-analysis. NA, information Not Available (gray cells). 
Supporting Table 3. Quality analysis of the nine datasets used for transcriptome meta-analysis. When available, raw data from Gene Expression Omnibus or Arrayexpress were normalized and $\log _{2}$ intensity expression summary values for each probe set were calculated using robust multiarray average (RMA). When raw data were not available, normalized data were directly used. Then, the probes expressed over the background in at least one of the HCCs were selected and genes were matched by ENTREZ ID. To assess the quality of each dataset, Pearson's correlation coefficients were measured for gene pairs expected to be correlated, as indicated in the table headings.

(cuppor

Supporting Table 4. Sanger sequencing of CTNNB1 exon 3 mutations in HCCs from the Training Set $(n=87)$, Validation Set $(n=56)$ and the external validation in-house HCC collection ( $n=82$; Rennes Hospital, France).

Supporting Table 5. Predicted CTNNB1 mutation rates in the nine datasets used for transcriptome meta-analysis. Gene expression data for GLUL, LGR5, ODAM, HAL and VNN1 were extracted from the training and validation sets and from the nine datasets ( $\mathrm{A}$ to I) and normalized with a median centering in each dataset. Then, the predictive scores were calculated (as shown in Fig. 2C) and the "best common threshold" maximizing sensitivity and specificity in both the training and the validation sets was optimized. In each of the nine datasets, samples with a score higher than the fixed threshold were predicted as carrying mutant CTNNB1. AUROC, area under the receiver operating curve. NA, not available. 
Supporting Table 6. Number and \% of HCCs predicted to carry CTNNB1 exon 3 mutations in each one of the transcriptomic subclasses (green, red, blue or purple) according to the individual datasets (A to I).

Supporting Table 7. Gene set expression analysis (GSEA) after classification of 1133 HCCs into four subclasses was performed with the web-based tool developed at the Broad Institute.(16)

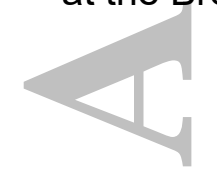

Supporting Table 8. Search for Gene Ontology (GO) terms overrepresented in each one of the gene clusters performed with the FuncAssociate 2.0(15) software using hgnc_symbol as namespace and $p<0.05$ as significance cutoff. $L O D$ is the $\log _{10}$ of the odds value indicating overrepresentation. Gene subsets were obtained after hierarchical clustering on the basis of the expression profile of the 550 top discriminant genes (VIP > 1.5 after PLS-DA analysis) in the metadata set.

Supporting Table 9. RNA expression levels of the 550 genes in the four transcriptomic subclasses of the metadata set. $P$ values were obtained by ANOVA. Cl: Confidence interval.

Supporting Table 10. Numbers and (\%) of HCCs in each one of the transcriptomic subclasses according to the individual datasets. PP, Periportal-type; PV, Perivenoustype; ECM, Extracellular-matrix-type; STEM, stem-cell-type. 
Supporting Table 11. Number and (\%) of HCCs in each one of the transcriptomic subclasses according to the sampling technique (tumor resection or biopsy) and etiology. Significantly predominant subclasses are highlighted in color.

Supporting Table 12. Quality analysis of RNAseq-based dataset extraction from the 210-HCC (TCGA-LICH-US) external validation set. Normalized data were extracted from the TCGA website. To assess the quality of dataset extraction, Pearson's correlation coefficients were measured for gene pairs expected to be correlated, as indicated in the table headings. Correlations are highlighted by a heatmap showing shades of red, indicating positive correlations and shades of blue, indicating negative correlations. The Periportal HCC Signature is highlighted in green, stem cell markers in purple, positive CTNNB1 mutation markers in red and negative CTNNB1 mutation markers in grey.

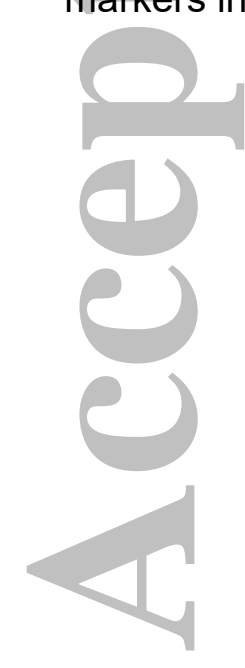




\section{REFERENCES}

1. Mebarki S, Desert R, Sulpice L, Sicard M, Desille M, Canal F, Schneider HD, et al. De novo HAPLN1 expression hallmarks Wnt-induced stem cell and fibrogenic networks leading to aggressive human hepatocellular carcinomas. Oncotarget 2016.

2. Leek JT, Johnson WE, Parker HS, Jaffe AE, Storey JD. The sva package for removing batch effects and other unwanted variation in high-throughput experiments. Bioinformatics 2012;28:882-883.

3. Le Cao KA, Rohart F, McHugh L, Korn O, Wells CA. YuGene: a simple approach to scale gene expression data derived from different platforms for integrated analyses. Genomics 2014;103:239-251.

4. Rohart F, Mason EA, Matigian N, Mosbergen R, Korn O, Chen T, Butcher S, et al. A molecular classification of human mesenchymal stromal cells. PeerJ 2016;4:e1845.

5. Hoshida Y, Nijman SM, Kobayashi M, Chan JA, Brunet JP, Chiang DY, Villanueva A, et al. Integrative transcriptome analysis reveals common molecular subclasses of human hepatocellular carcinoma. Cancer research 2009;69:73857392.

6. Lachenmayer A, Alsinet C, Savic R, Cabellos L, Toffanin S, Hoshida Y, Villanueva A, et al. Wnt-pathway activation in two molecular classes of hepatocellular carcinoma and experimental modulation by sorafenib. Clinical cancer research : an official journal of the American Association for Cancer Research 2012;18:4997-5007. 7. Zucman-Rossi J, Benhamouche S, Godard C, Boyault S, Grimber G, Balabaud C, Cunha AS, et al. Differential effects of inactivated Axin1 and activated beta-catenin mutations in human hepatocellular carcinomas. Oncogene 2007;26:774780. 
8. Yamamoto Y, Sakamoto M, Fujii G, Tsuiji H, Kenetaka K, Asaka M, Hirohashi

S. Overexpression of orphan G-protein-coupled receptor, Gpr49, in human hepatocellular carcinomas with beta-catenin mutations. Hepatology 2003;37:528533.

9. Filzmoser P, Walczak B. What can go wrong at the data normalization step for identification of biomarkers? Journal of chromatography. A 2014;1362:194-205.

10. de La Coste A, Romagnolo B, Billuart P, Renard CA, Buendia MA, Soubrane $\mathrm{O}$, Fabre $\mathrm{M}$, et al. Somatic mutations of the beta-catenin gene are frequent in mouse and human hepatocellular carcinomas. Proc Natl Acad Sci U S A 1998;95:88478851.

11. Zucman-Rossi J, Villanueva A, Nault JC, Llovet JM. Genetic Landscape and Biomarkers of Hepatocellular Carcinoma. Gastroenterology 2015;149:1226-1239 e1224.

12. Govaere O, Komuta M, Berkers J, Spee B, Janssen C, de Luca F, Katoonizadeh A, et al. Keratin 19: a key role player in the invasion of human hepatocellular carcinomas. Gut 2014;63:674-685.

13. Boyault S, Rickman DS, de Reynies A, Balabaud C, Rebouissou S, Jeannot E, Herault A, et al. Transcriptome classification of HCC is related to gene alterations and to new therapeutic targets. Hepatology 2007;45:42-52.

14. Désert R, Canlet, C., Costet, N., Cordier, S., Bonvallot, N. Impact of maternal obesity on the metabolic profiles of pregnant women and their offspring at birth. Metabolomics 2015;11:1896-1907.

15. Berriz GF, Beaver JE, Cenik C, Tasan M, Roth FP. Next generation software for functional trend analysis. Bioinformatics 2009;25:3043-3044. 
16. Subramanian A, Tamayo P, Mootha VK, Mukherjee S, Ebert BL, Gillette MA, Paulovich A, et al. Gene set enrichment analysis: a knowledge-based approach for interpreting genome-wide expression profiles. Proceedings of the National Academy of Sciences of the United States of America 2005;102:15545-15550.

17. Gougelet A, Torre C, Veber P, Sartor C, Bachelot L, Denechaud PD, Godard C, et al. T-cell factor 4 and beta-catenin chromatin occupancies pattern zonal liver metabolism in mice. Hepatology 2014;59:2344-2357.

18. Roessler S, Jia HL, Budhu A, Forgues M, Ye QH, Lee JS, Thorgeirsson SS, et al. A unique metastasis gene signature enables prediction of tumor relapse in earlystage hepatocellular carcinoma patients. Cancer research 2010;70:10202-10212.

19. Mebarki S, Desert R, Sulpice L, Sicard M, Desille M, Canal F, Dubois-Pot Schneider H, et al. De novo HAPLN1 expression hallmarks Wnt-induced stem cell and fibrogenic networks leading to aggressive human hepatocellular carcinomas. Oncotarget 2016;7:39026-39043.

20. Desert R, Mebarki S, Desille M, Sicard M, Lavergne E, Renaud S, Bergeat D, et al. "Fibrous nests" in human hepatocellular carcinoma express a Wnt-induced gene signature associated with poor clinical outcome. The international journal of biochemistry \& cell biology 2016 .

21. Le Jossic C, Glaise D, Corcos L, Diot C, Dezier JF, Fautrel A, GuguenGuillouzo C. trans-Acting factors, detoxication enzymes and hepatitis B virus replication in a novel set of human hepatoma cell lines. Eur $\mathrm{J}$ Biochem 1996;238:400-409.

22. Cerec V, Glaise D, Garnier D, Morosan S, Turlin B, Drenou B, Gripon P, et al. Transdifferentiation of hepatocyte-like cells from the human hepatoma HepaRG cell line through bipotent progenitor. Hepatology 2007;45:957-967. 
23. Dubois-Pot-Schneider H, Fekir K, Coulouarn C, Glaise D, Aninat C, Jarnouen

$\mathrm{K}$, Le Guevel R, et al. Inflammatory cytokines promote the retrodifferentiation of tumor-derived hepatocyte-like cells to progenitor cells. Hepatology 2014;60:20772090.

24. Colletti M, Cicchini C, Conigliaro A, Santangelo L, Alonzi T, Pasquini E, Tripodi $M$, et al. Convergence of Wnt signaling on the HNF4alpha-driven transcription in controlling liver zonation. Gastroenterology 2009;137:660-672.

25. Wang B, Zhao L, Fish M, Logan CY, Nusse R. Self-renewing diploid Axin2(+) cells fuel homeostatic renewal of the liver. Nature 2015;524:180-185.

26. Johnson WE, Li C, Rabinovic A. Adjusting batch effects in microarray expression data using empirical Bayes methods. Biostatistics 2007;8:118-127.

27. Muller PA, Vousden KH. p53 mutations in cancer. Nature cell biology 2013;15:2-8.

28. Fei Q, Shang K, Zhang J, Chuai S, Kong D, Zhou T, Fu S, et al. Histone methyltransferase SETDB1 regulates liver cancer cell growth through methylation of p53. Nature communications 2015;6:8651.

29. Holloway MG, Miles GD, Dombkowski AA, Waxman DJ. Liver-specific hepatocyte nuclear factor-4alpha deficiency: greater impact on gene expression in male than in female mouse liver. Molecular endocrinology 2008;22:1274-1286.

30. Wu C, Jin X, Tsueng G, Afrasiabi C, Su Al. BioGPS: building your own mashup of gene annotations and expression profiles. Nucleic acids research 2016;44:D313-316.

31. Hsu HC, Jeng YM, Mao TL, Chu JS, Lai PL, Peng SY. Beta-catenin mutations are associated with a subset of low-stage hepatocellular carcinoma negative for 
hepatitis B virus and with favorable prognosis. The American journal of pathology 2000;157:763-770.

32. Nault JC, De Reynies A, Villanueva A, Calderaro J, Rebouissou S, Couchy G, Decaens $\mathrm{T}$, et al. A hepatocellular carcinoma 5-gene score associated with survival of patients after liver resection. Gastroenterology 2013;145:176-187.

33. Rebouissou S, Franconi A, Calderaro J, Letouze E, Imbeaud S, Pilati C, Nault JC, et al. Genotype-phenotype correlation of CTNNB1 mutations reveals different sscatenin activity associated with liver tumor progression. Hepatology 2016.

34. Kan Z, Zheng H, Liu X, Li S, Barber TD, Gong Z, Gao H, et al. Whole-genome sequencing identifies recurrent mutations in hepatocellular carcinoma. Genome research 2013;23:1422-1433.

35. Guichard C, Amaddeo G, Imbeaud S, Ladeiro Y, Pelletier L, Maad IB, Calderaro J, et al. Integrated analysis of somatic mutations and focal copy-number changes identifies key genes and pathways in hepatocellular carcinoma. Nature genetics 2012;44:694-698.

36. Yuan RH, Jeng YM, Hu RH, Lai PL, Lee PH, Cheng CC, Hsu HC. Role of p53 and beta-catenin mutations in conjunction with CK19 expression on early tumor recurrence and prognosis of hepatocellular carcinoma. Journal of gastrointestinal surgery : official journal of the Society for Surgery of the Alimentary Tract 2011;15:321-329. 


\section{Exalepaiøogeriteria}

Page 130 of 143

A

- Cholangiocarcinoma $(n=1)$

- Fibrolamellar hepatocellular carcinoma $(\mathrm{n}=2)$

- Mixed hepatocellular / cholangiocarcinoma $(n=4)$

\section{Available HCC samples}

Isith RNAseq and clinical annotation data $\begin{array}{r}11 \\ 12 \\ \hline 13\end{array}$ $(n=294)$

- Unsure diagnosis $(n=1)$

- Low sample quality $(n=2)$

- No vital annotation ( $n=36)$

- Patient treated by liver transplantation $(\mathrm{n}=1)$

- History of prior malignancy $(n=19)$

- New primary tumor $(n=7)$

- Early death (< 90 days post resection, $n=11$ )

\section{Analysed samples $(n=210)$}

B
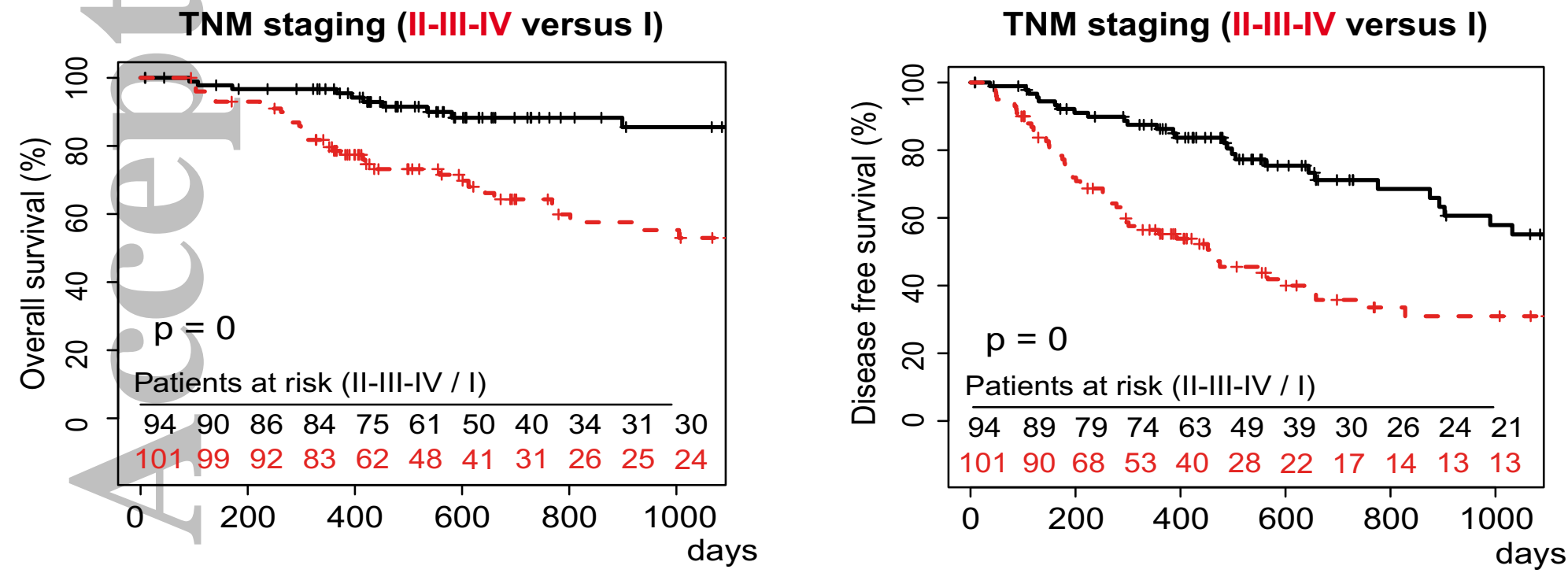

Hepatology

This article is protected by copyright. All rights reserved.

Désert et al., Supporting Figure 1 
Training set

Hepatology

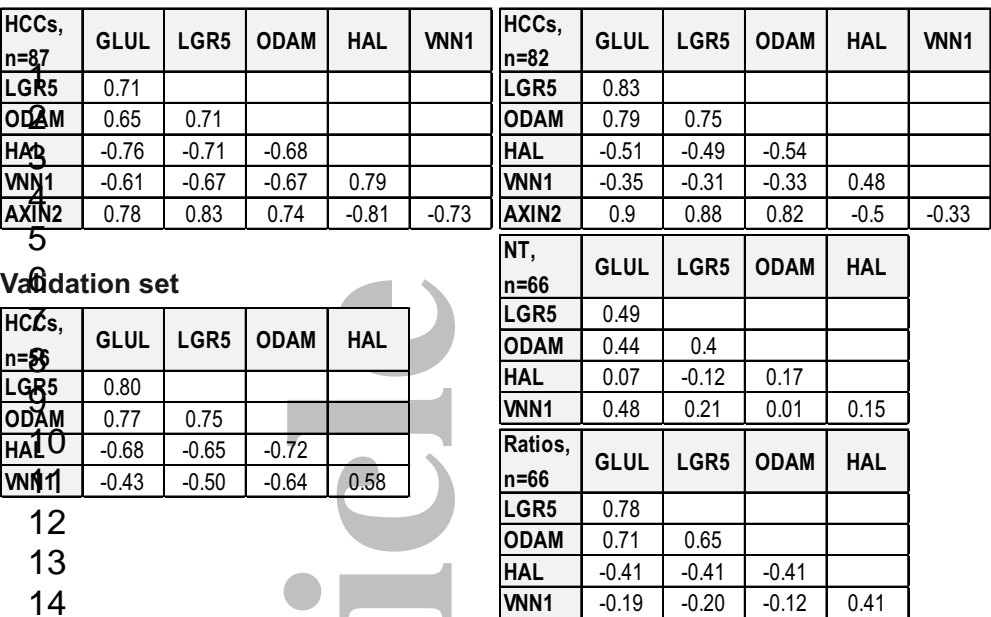

15

$B^{16}$

17

18

19

20

21

22

23

24

25

26

27

28

c29

$\mathrm{C}_{30}$

31

32

33

34

35

36

37

38

39

40

41

42

$D_{43}$

44

45

H

va

C\$S

L6t

\section{HAS}

$$
\begin{aligned}
& \text { Wis } \\
& \begin{array}{l}
5 \\
\hline 5 \\
5 \\
5
\end{array}
\end{aligned}
$$

55

5

54

55

56

57

58

59

60
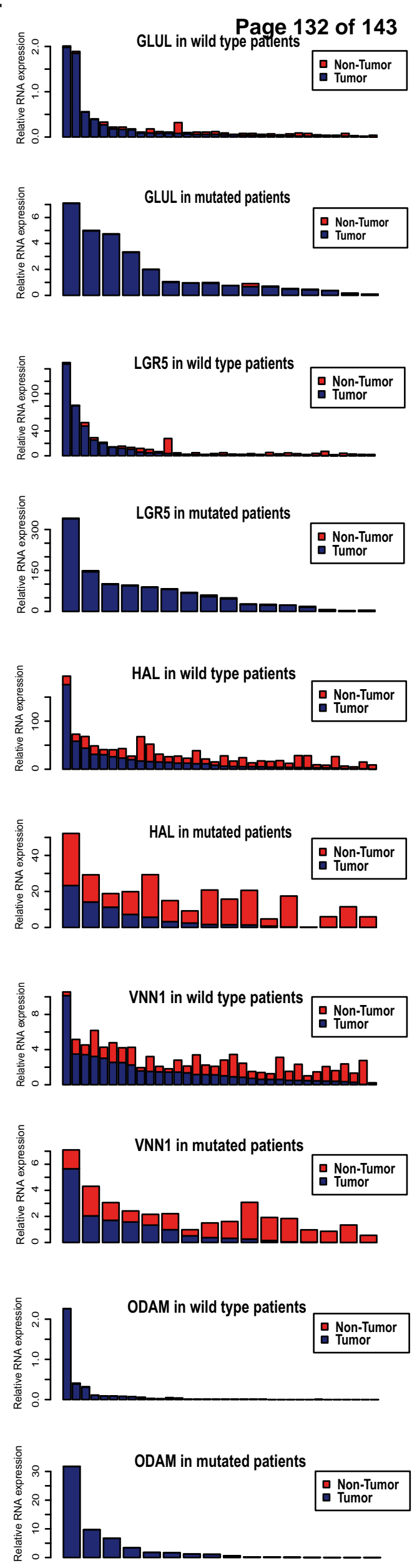

\section{Hepatology}

This article is protected by copyright. All rights reserved. Désert et al., Supporting Figure 3 


\section{Page 133 of 143 Hepatology}
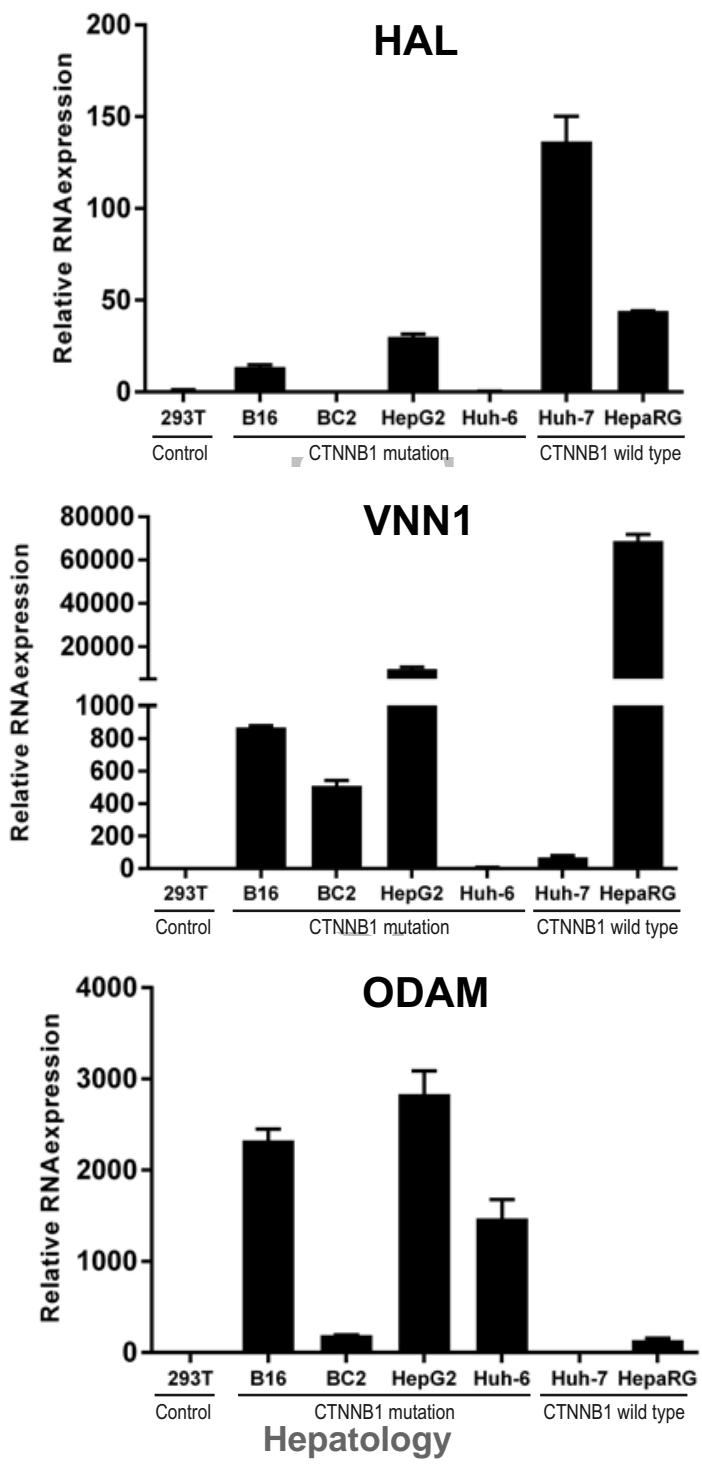

This artic

Désert et al., Supporting Figure 4 
A

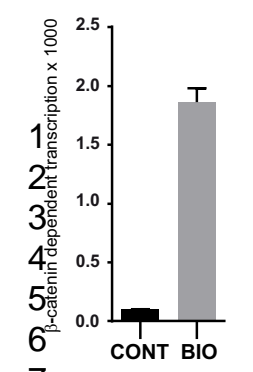

B 7 ${ }_{8} \mathrm{E}$

$\left.9={ }^{15}\right]$ CONTROL

19 㷓 12 1 พె⿱ $1 \frac{4}{4}$ 15 16 17
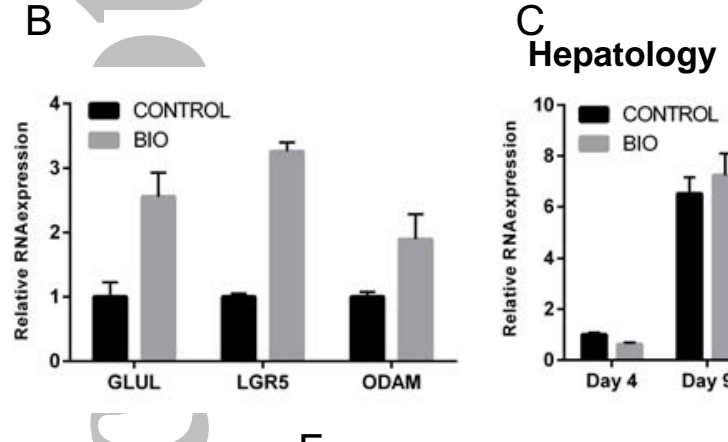

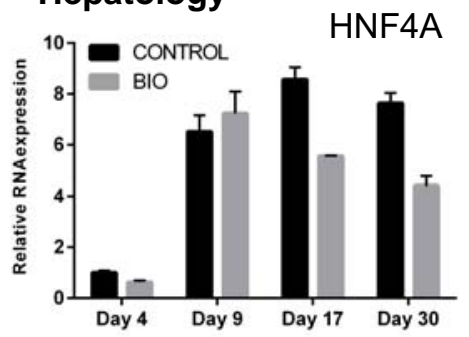

Page 134 of 143 ALDOB

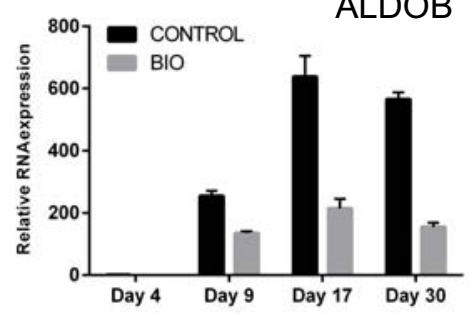

F
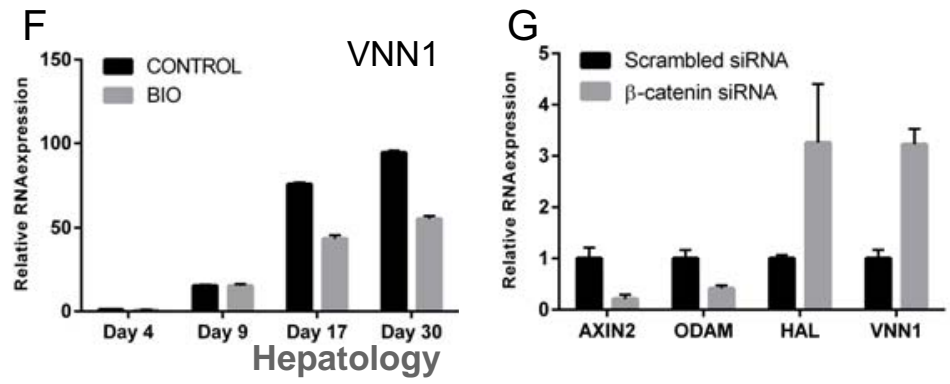

This article is protected by copyright. All rights reserve Désert et al., Supporting Figure 5 


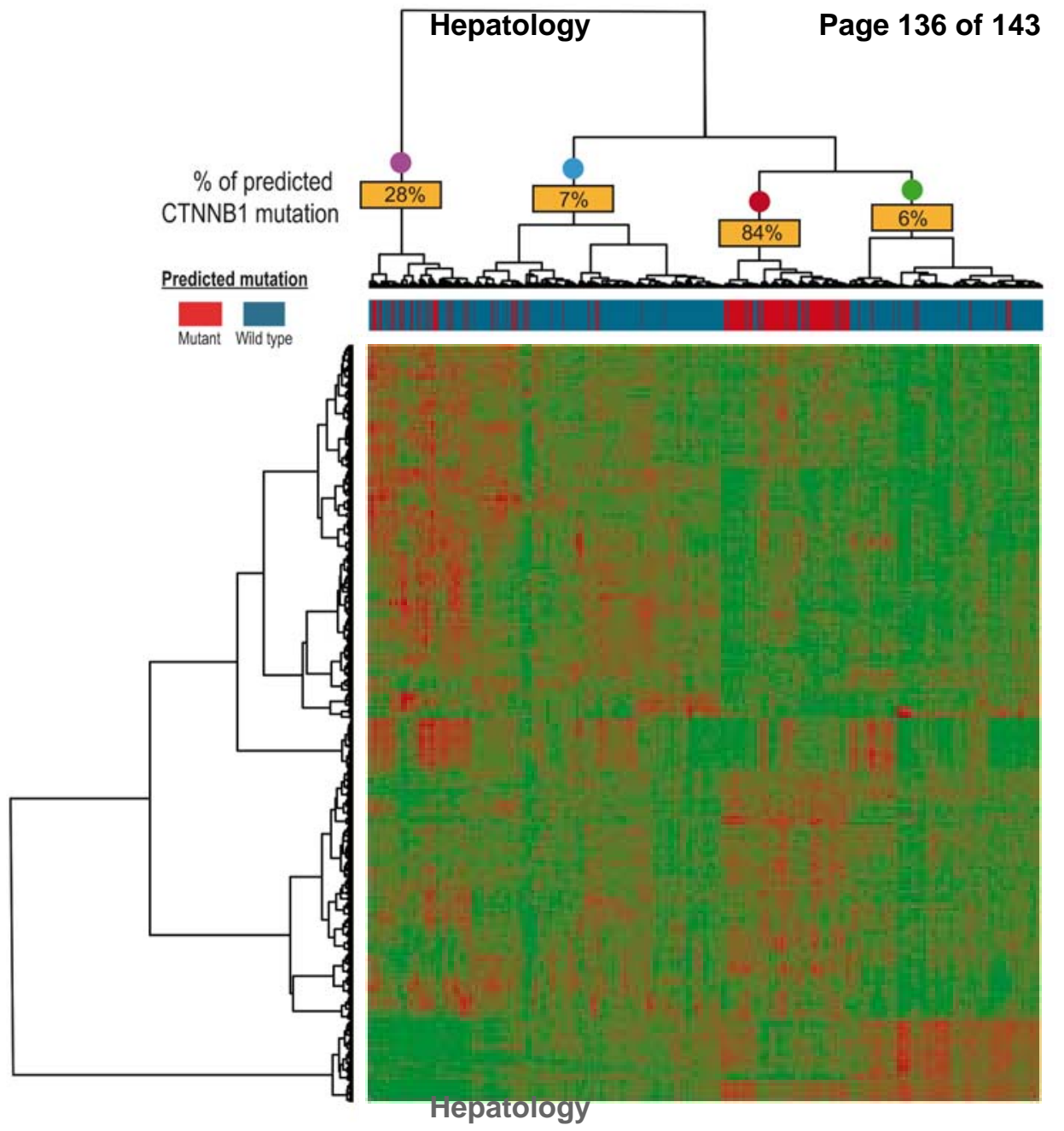

This article is protected by copyright. All $\mathrm{n}$ Désert et al., Supporting Figure 7 


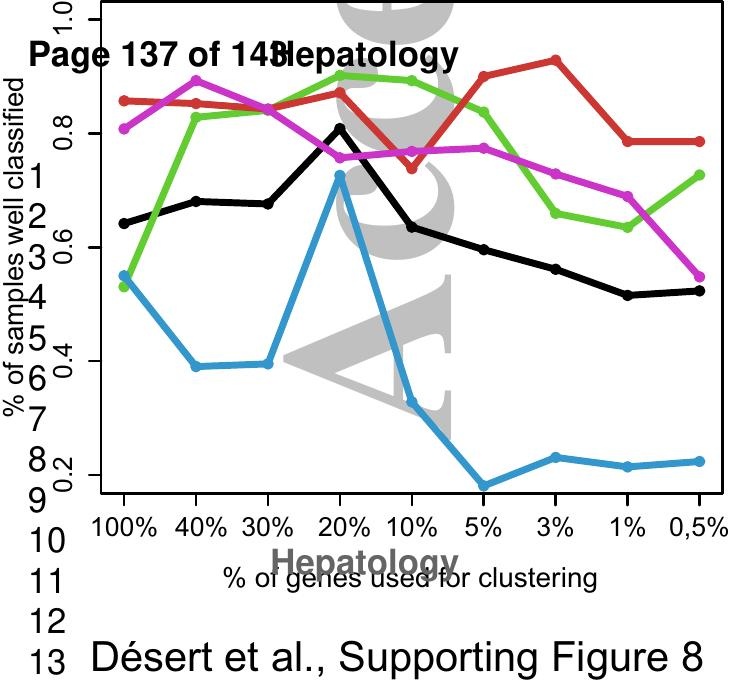


A

\section{Genes}

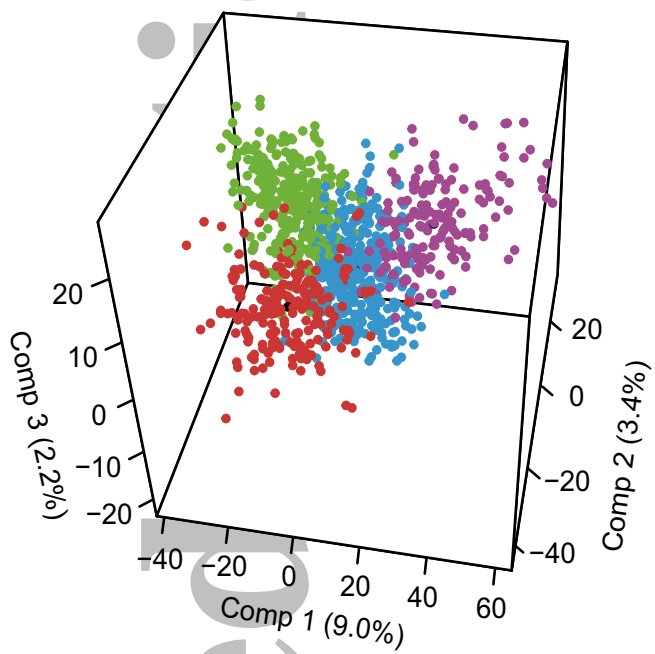

D

\begin{tabular}{|c|c|c|c|c|c|}
\hline & \multicolumn{4}{|c|}{ 1618-gene clustering } \\
\hline & & Red & Purple & Green & Blue \\
\hline \multirow{4}{*}{ 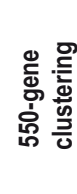 } & Red & 182 & 0 & 14 & 8 \\
\hline & Purple & 6 & 176 & 0 & 77 \\
\hline & Green & 11 & 0 & 291 & 59 \\
\hline & Blue & 11 & 1 & 21 & 276 \\
\hline
\end{tabular}

Sum : $182+176+291+276=925=81.6 \%$

3
3
3
3
4
4
4
4
4
4
4
4
4
5
5
5
5
5
5
5
5
5
5
6

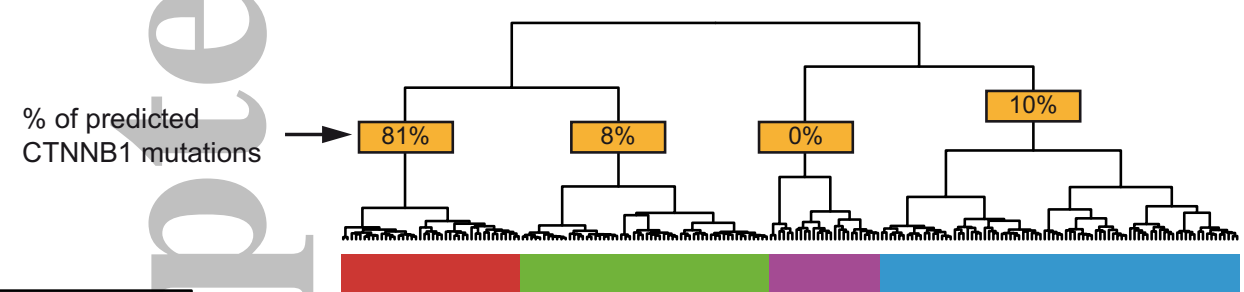

Samples

${ }_{37}^{36}$ Green $(n=140)$

3\&Red ( $n=104)$

399lue/Purple ( $n=205)$

40

41

42

43

44

45

46

47

Green ( $n=58)$

Red $(n=42)$

- Blue $(n=84)$

Purple $(\mathrm{n}=26)$ 1 3

5

5
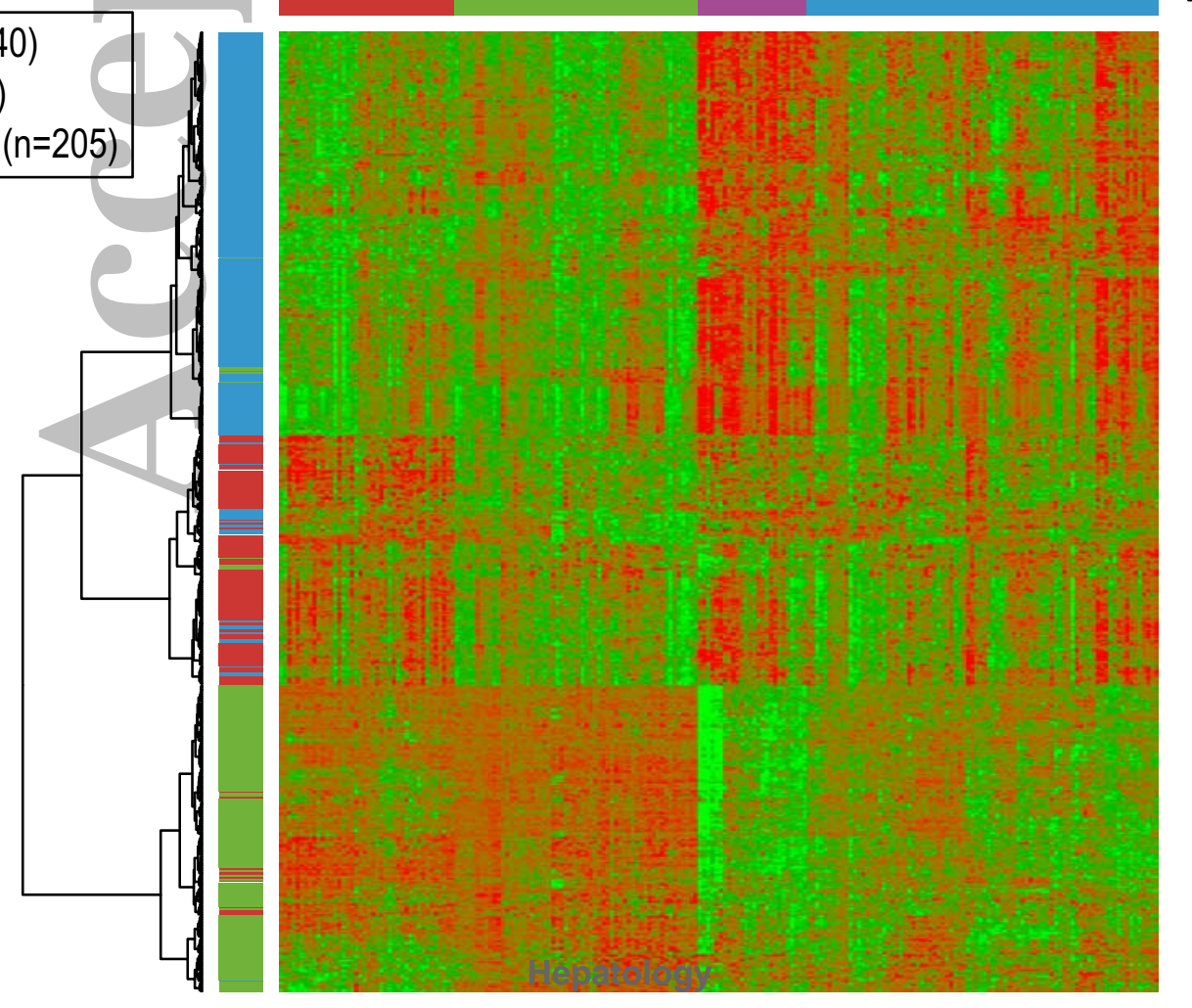

This article is protected by copyright. All rights reserved. Désert et al., Supporting Figure 9 
Hepatology

GLS2
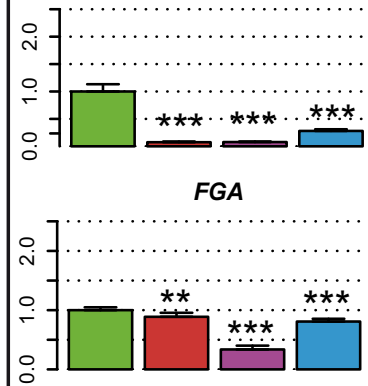

ALB

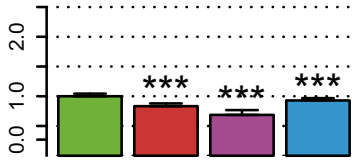

EGFR

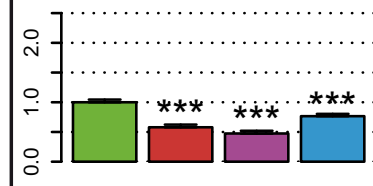

Page $_{F B}{ }^{4} f 0$ of 143

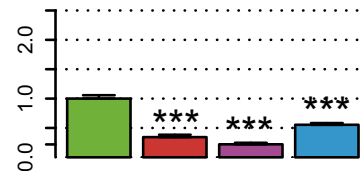

ALDOB

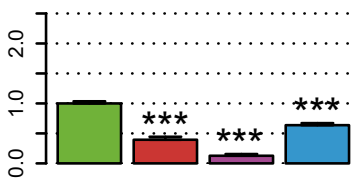

CDH1

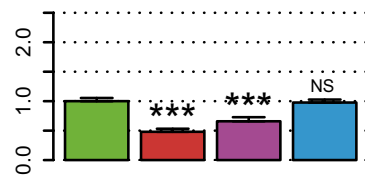

AQP8

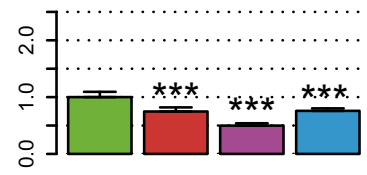

AFP

29

$3 \theta$

ogt

3

3

35

3
3
3
3
4
40

41

4

43 $\star \star * * * *$

\section{2}
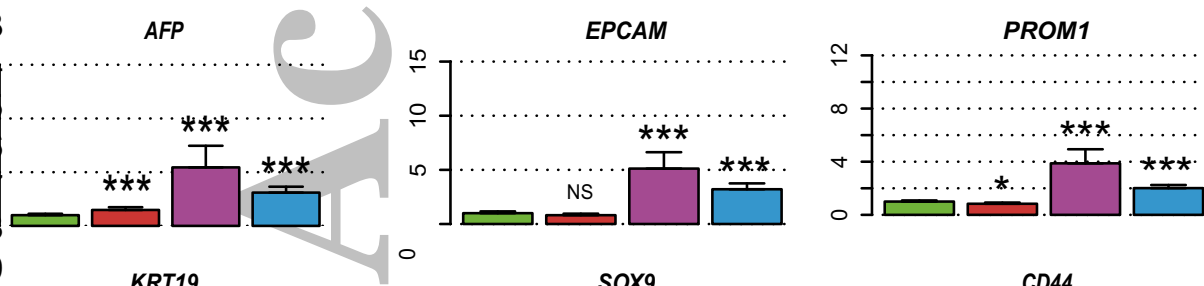

CD44

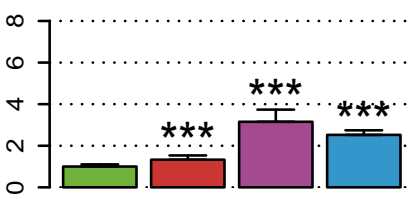

TNC
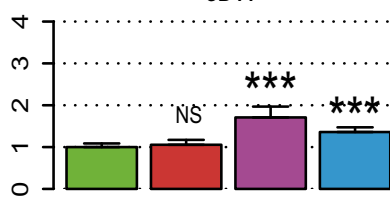

LAMC1

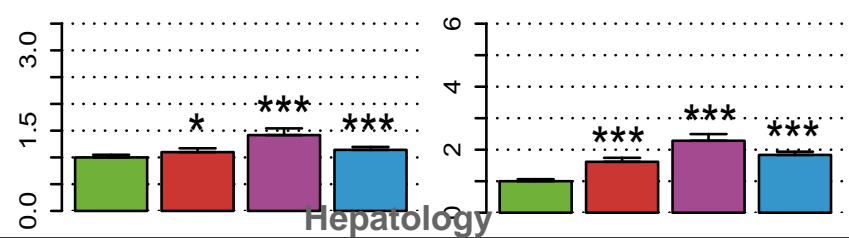

This article is protected by copyright. All rights reserved. Désert et al., Supporting Figure 11

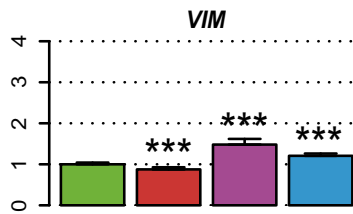

VCAN

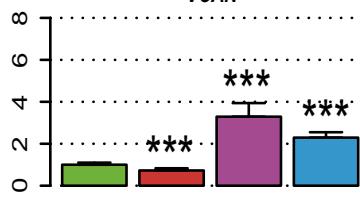

TGFB1

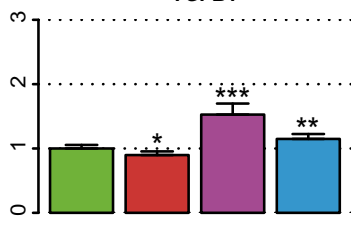


A

Page 141 of 143
PV (210 tumors; 79 genes)

Hepatology

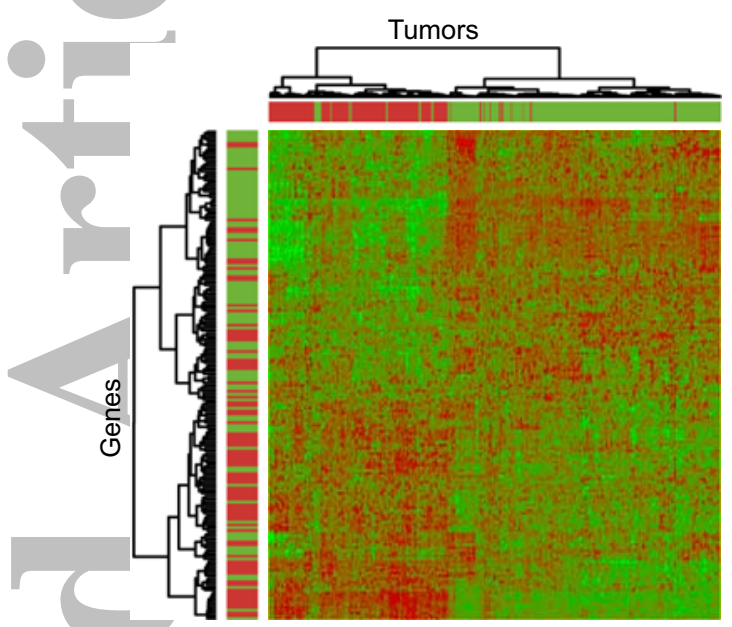

B
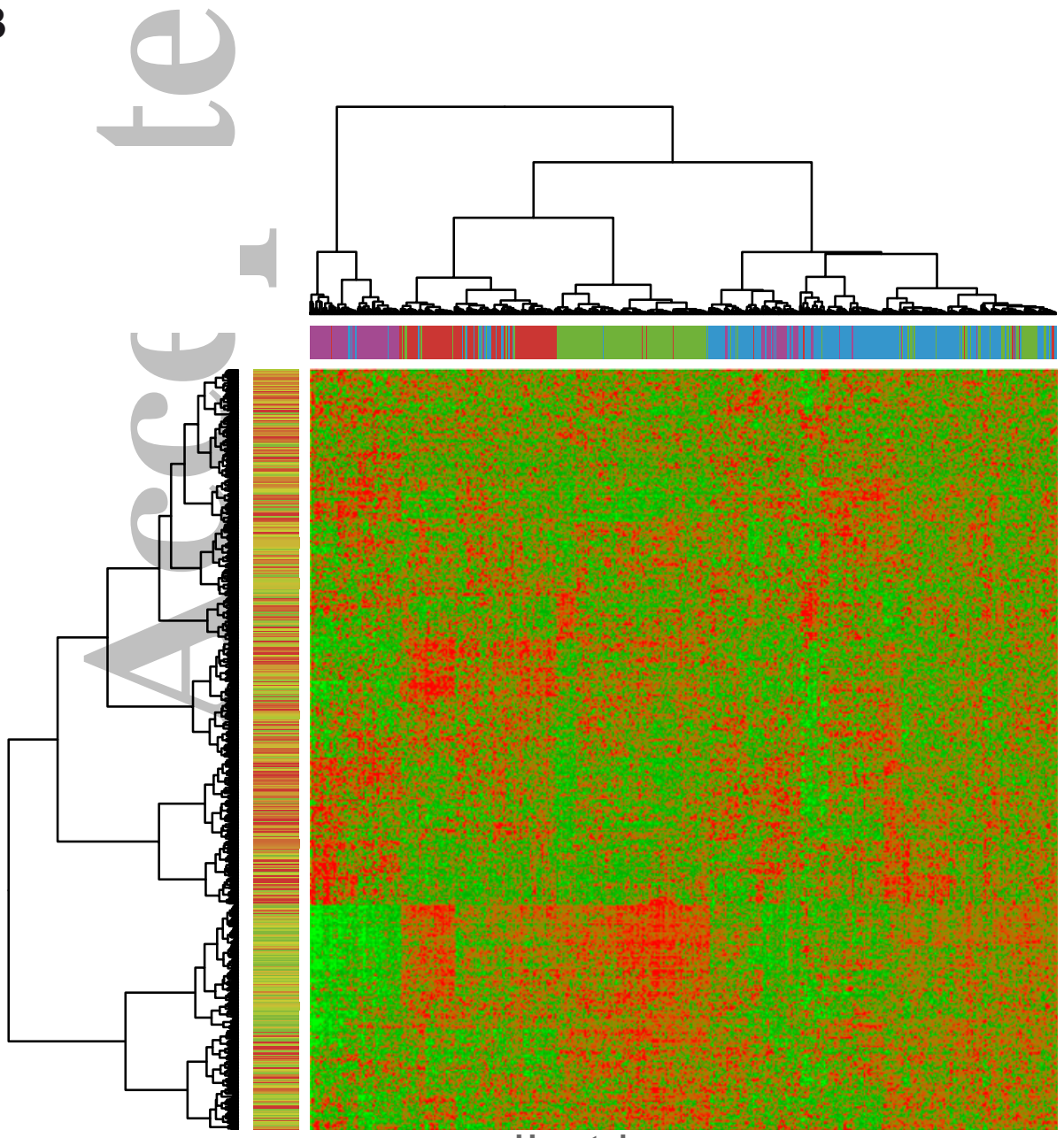

Genes (fold change HNF4 KO / WT) :

-0.01
-0.14
-0.25
-0.35
-0.42
-0.49
-2.15
-2.53
-3.18
-4.77

Tumor (subclass) :

- $P P(n=326)$

- $P V(n=210)$

- ECM $(n=420)$

- STEM $(n=177)$

\section{Hepatology}

This article is protected by copyright. All rights reserved. Désert et al., Supporting Figure 12 


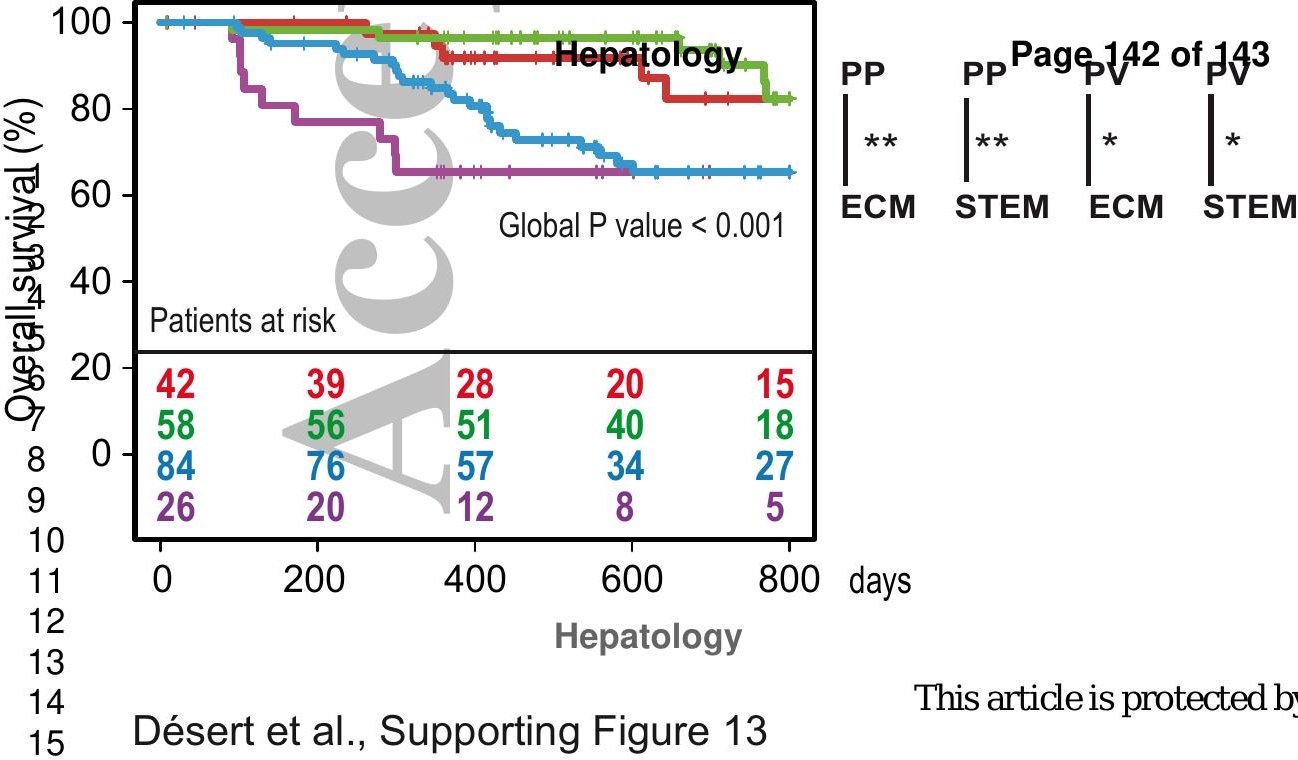




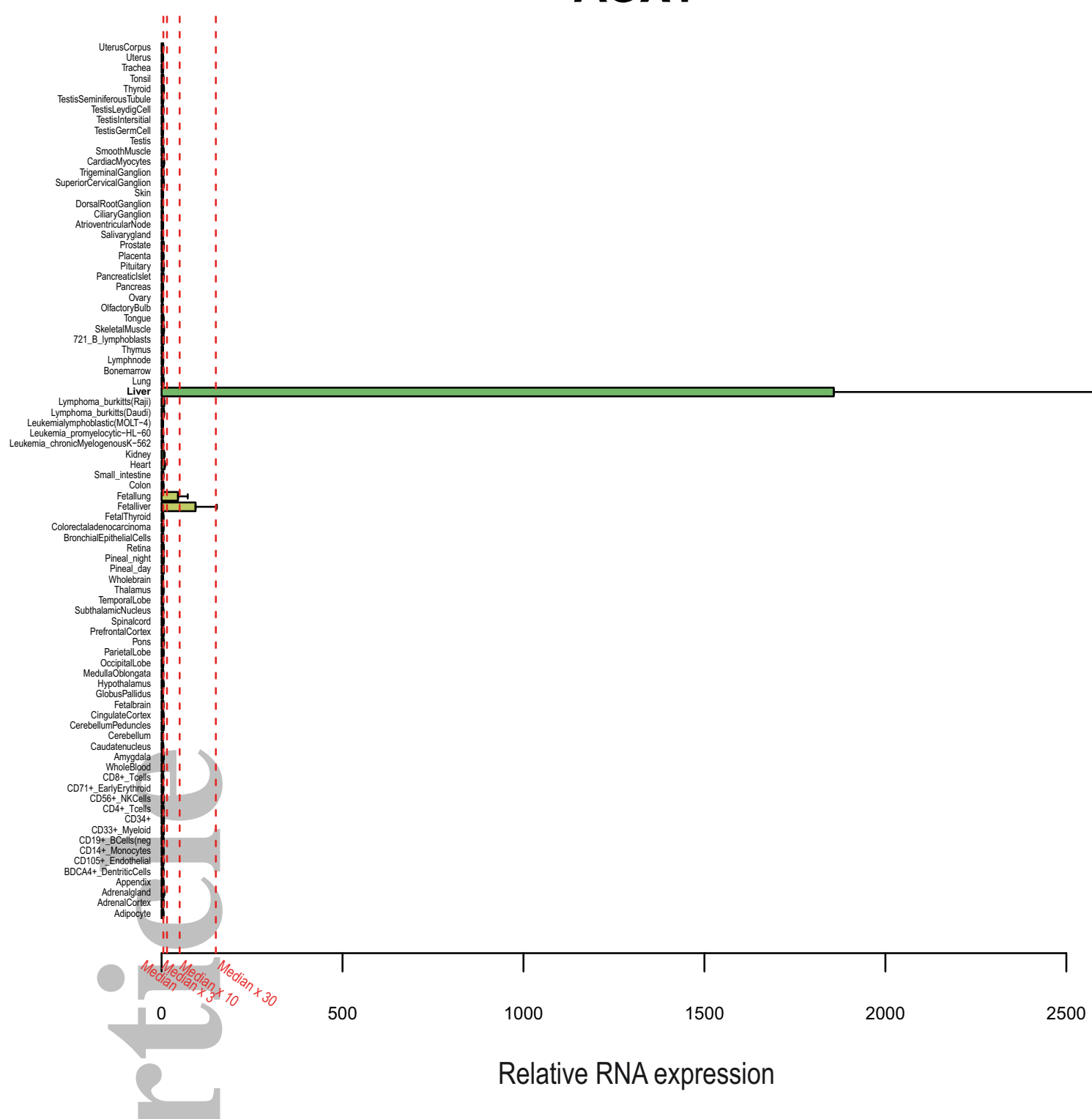

\section{FETUB}

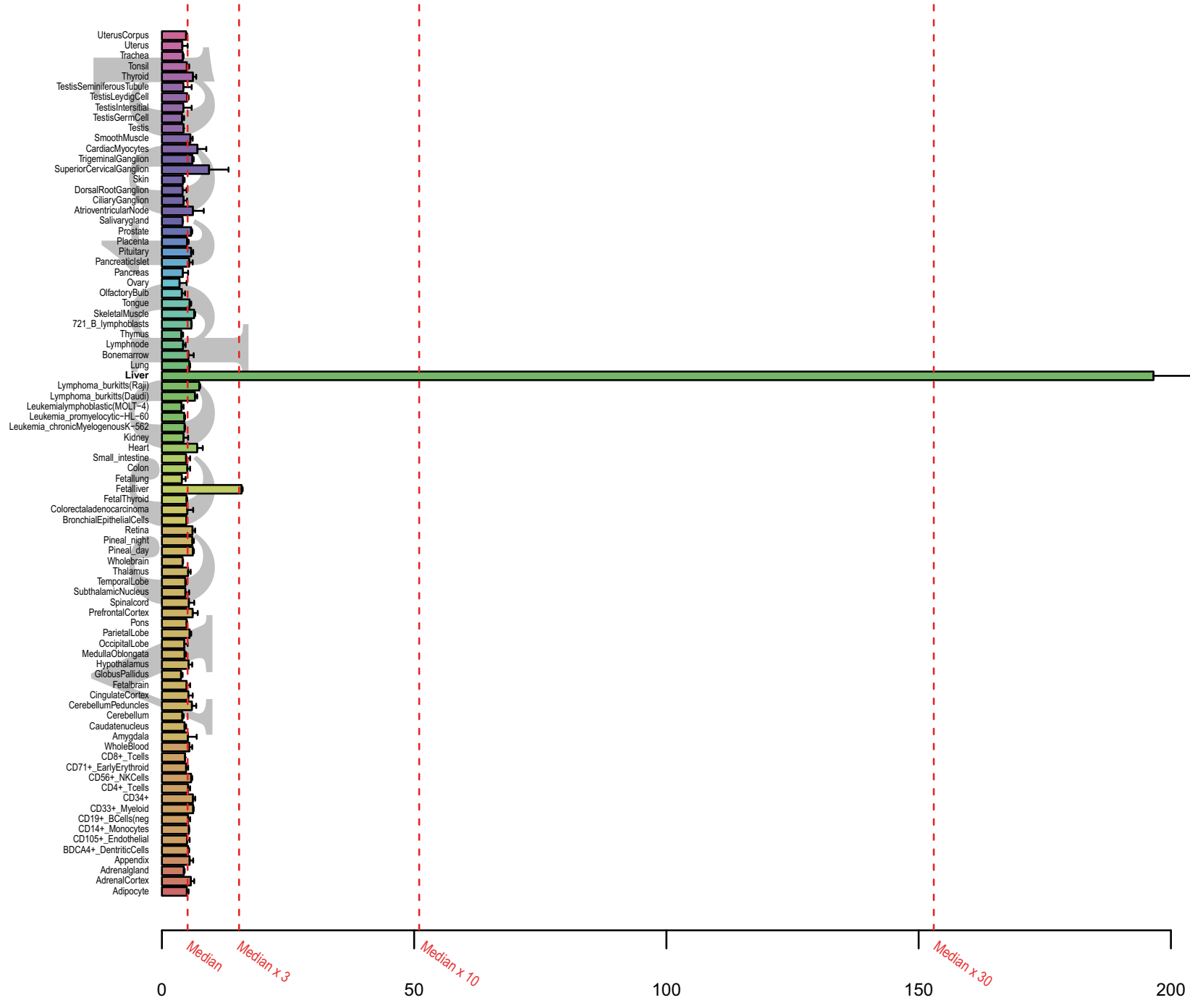

Relative RNA expression Hepatology

This article is protected by copyright. All rights reserved. Désert et al., Supporting Figure 14, A \& B 

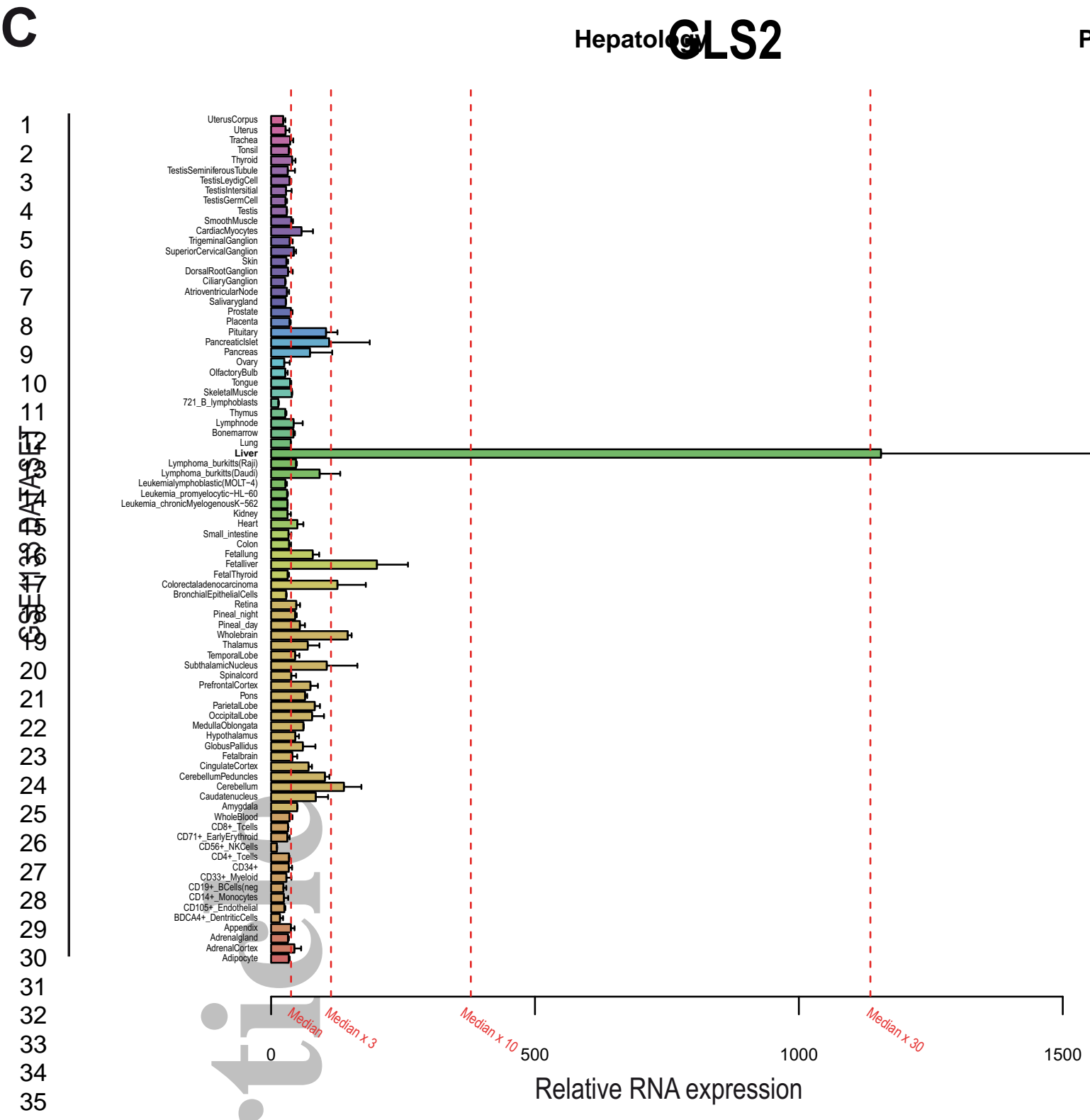

\section{GNMT}

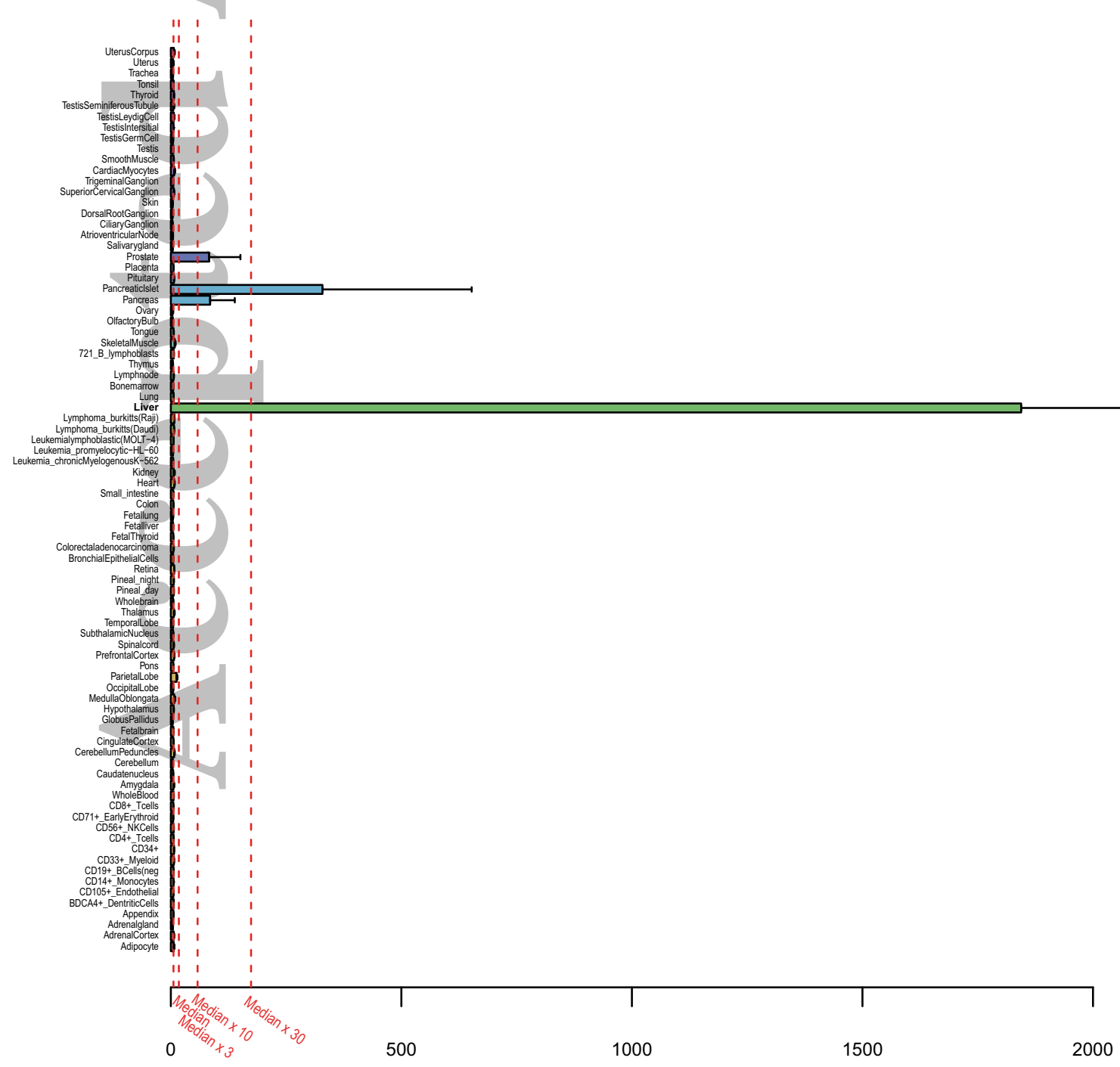

Relative RNA expression

Hepatology

This article is protected by copyright. All rights reserved. Désert et al., Supporting Figure $14, C$ \& D 


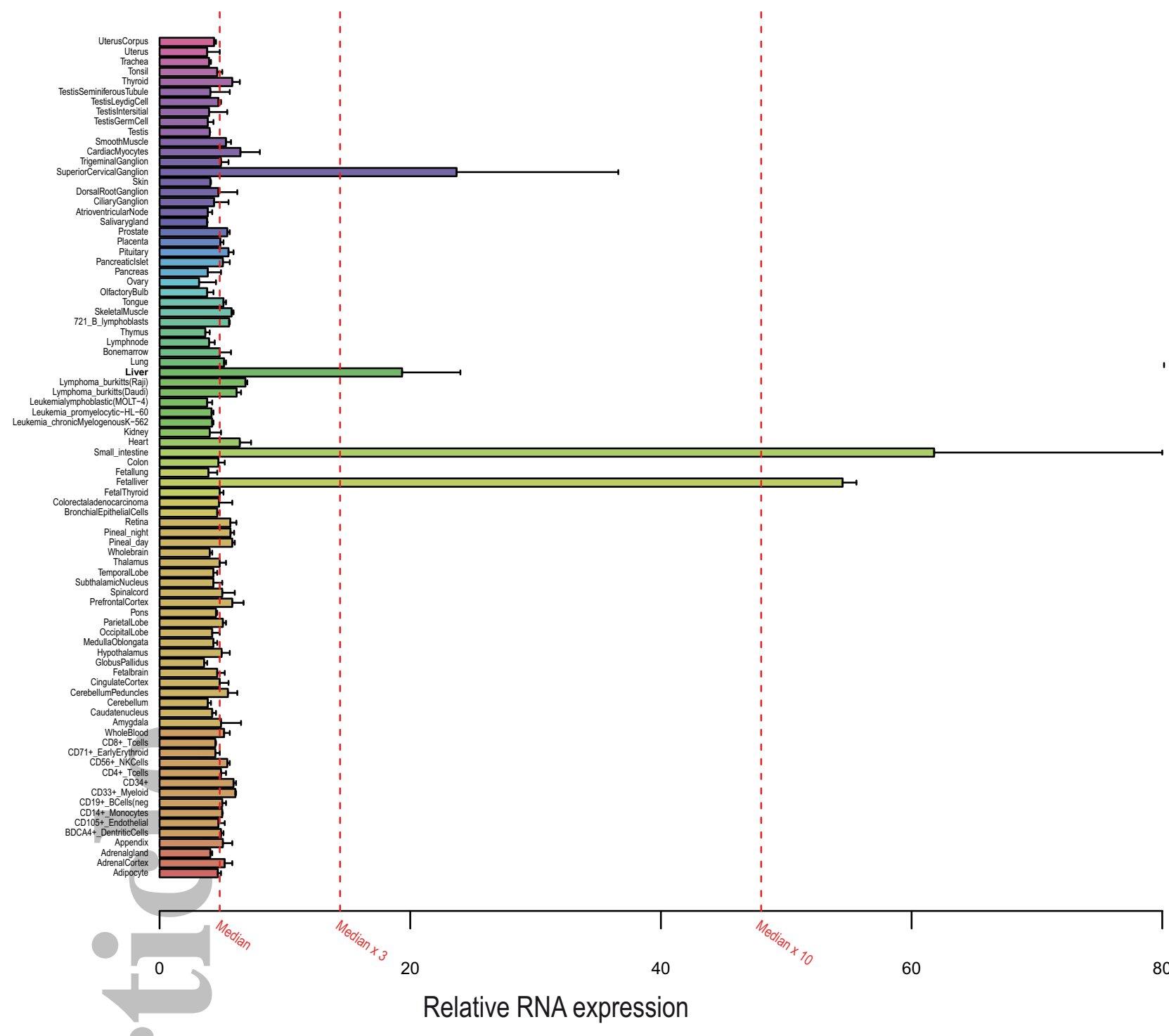

\section{SLC10A1}

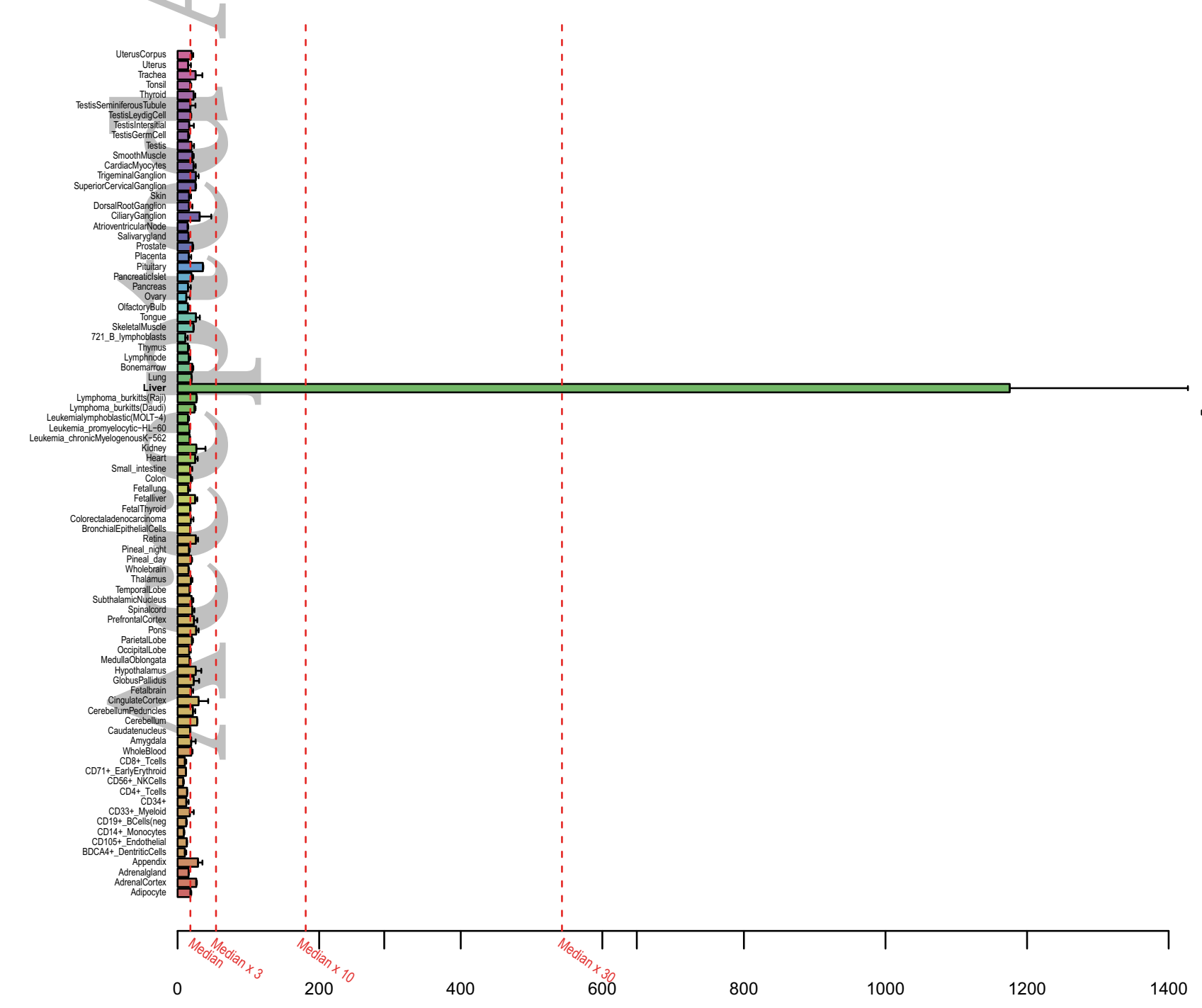

Relative RNA expression

Hepatology

This article is protected by copyright. All rights reserved. Désert et al., Supporting Figure $14, \mathrm{E}$ \& F 


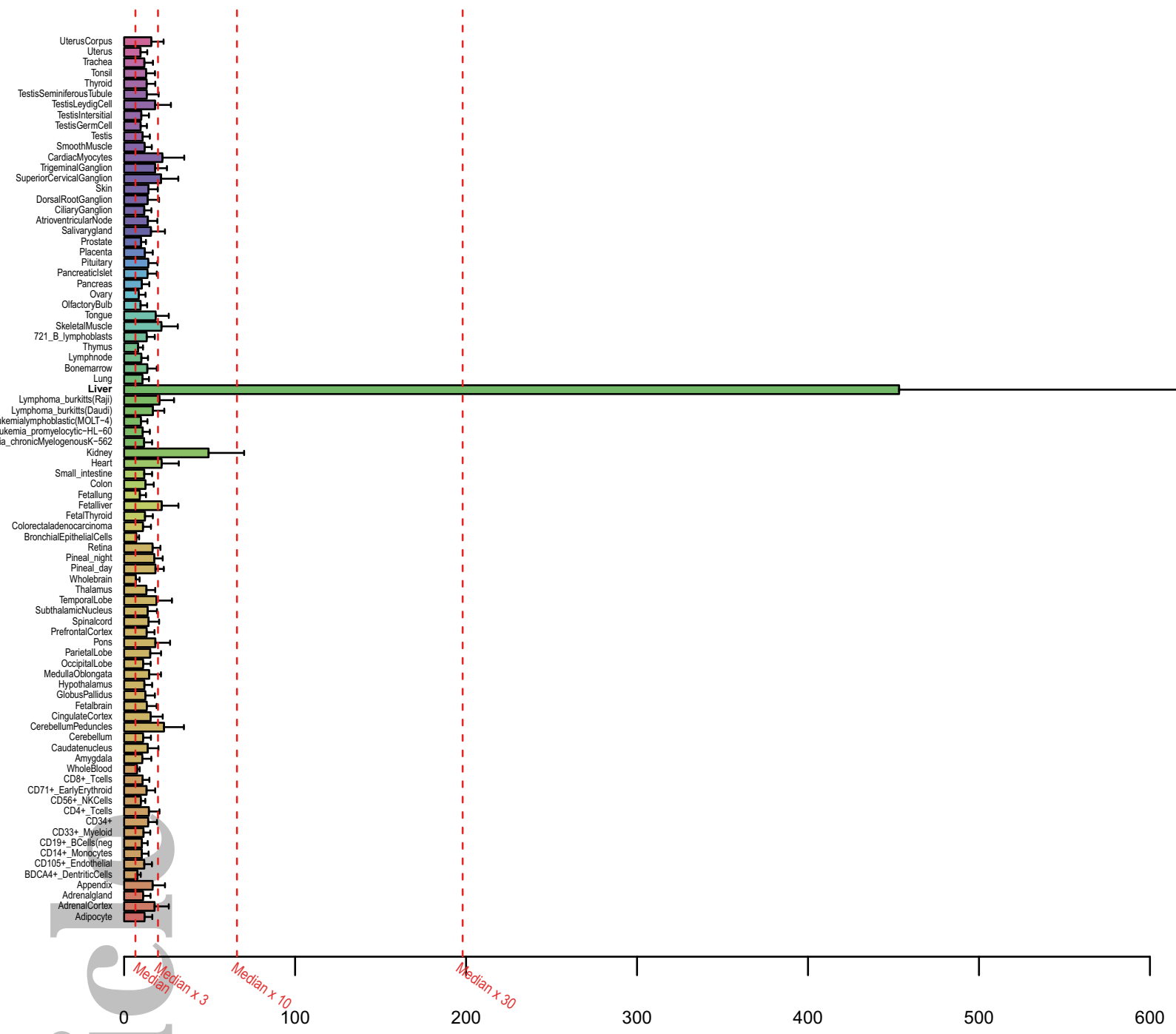

Relative RNA expression

\section{SLC27A5}

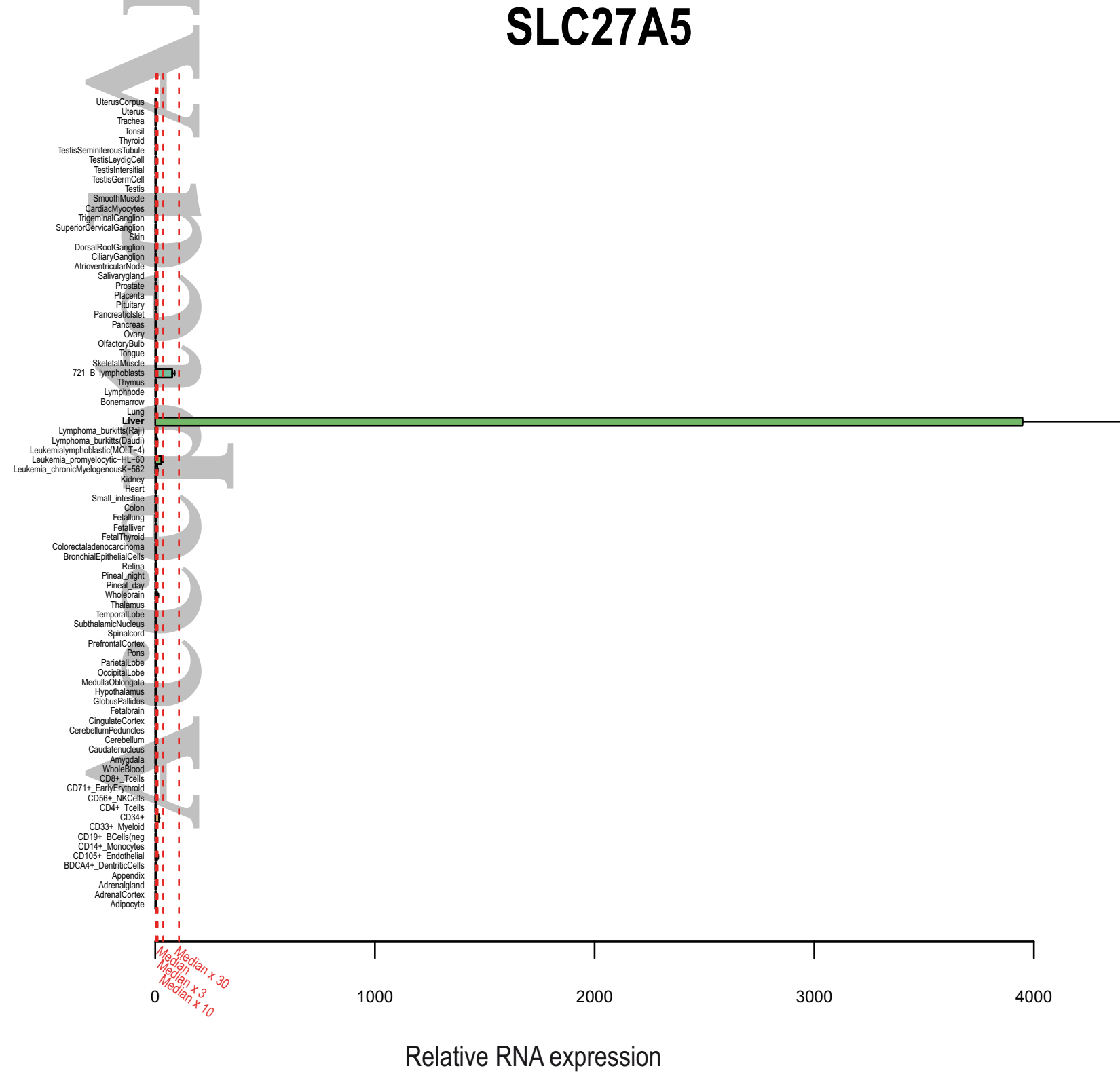

Hepatology

This article is protected by copyright. All rights reserved. Désert et al., Supporting Figure $14, \mathrm{G} \& \mathrm{H}$ 


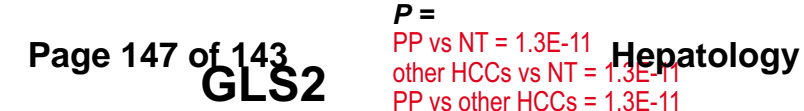

PP vs other HCCs = 1.3E-11 FETUB

$\boldsymbol{P}=$
PP vs NT $=1.2 \mathrm{E}-02$

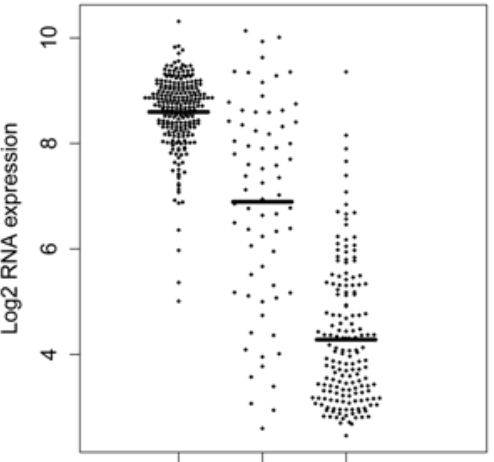

NT PP other HCCs

$\boldsymbol{P}=$

PP vs NT $=7.3 \mathrm{E}-06$ SLC22A7 $\begin{aligned} & \text { other HCCs vs NT }=1.3 \mathrm{E}-11 \\ & \text { PP vs other HCCs }=1.3 \mathrm{E}-11\end{aligned}$

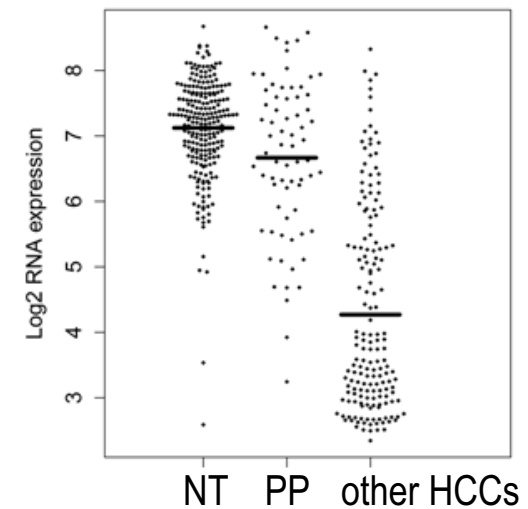

NT PP other HCCs
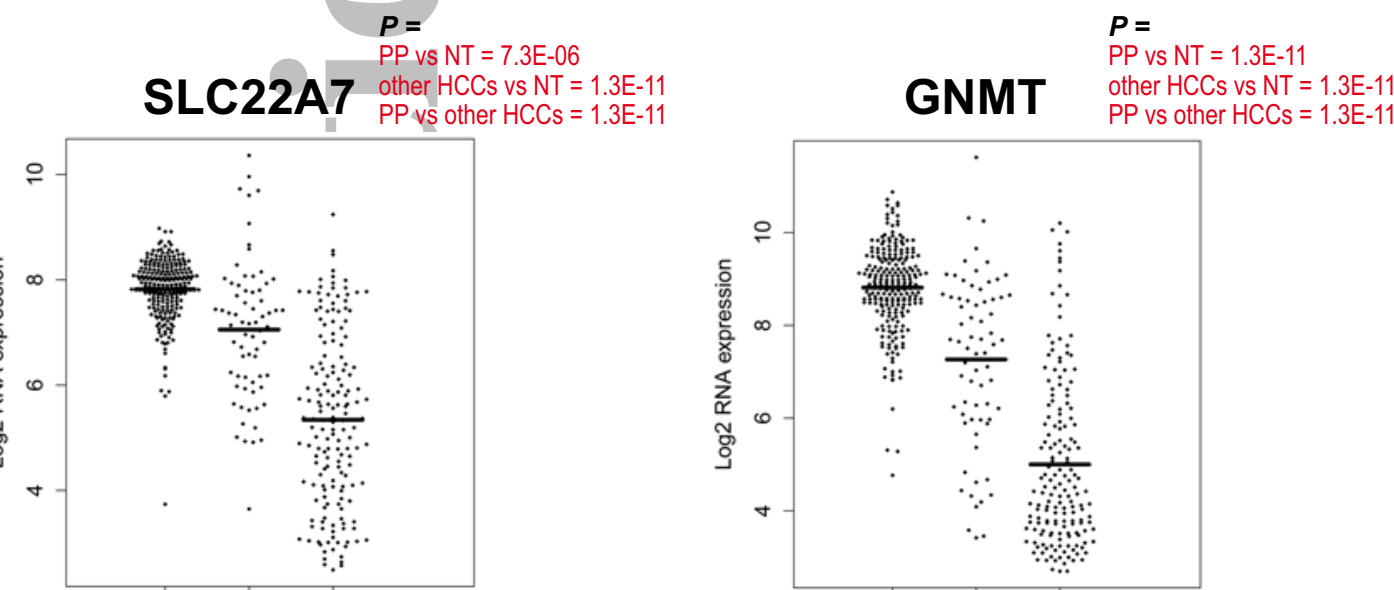

NT PP" other HCCs

$P=$

PP vs NT= 3.7E-04

SLC10A1 1 ther HCCs vs NT = 1.3E-11

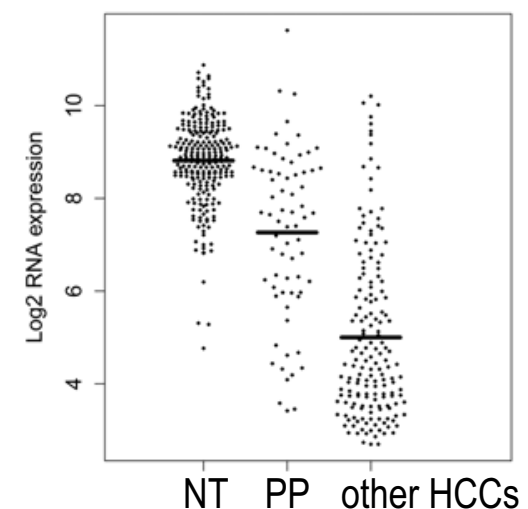

NT PP other HCCs

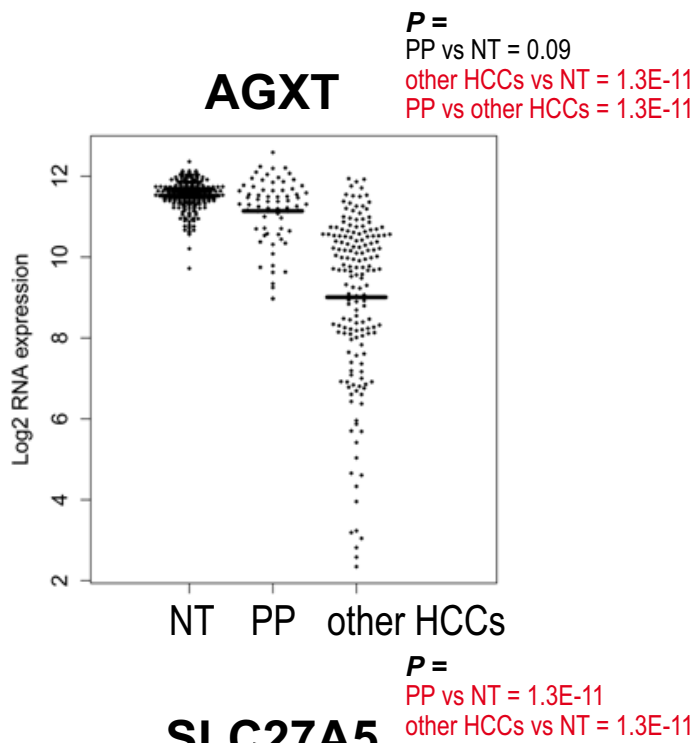

SLC27A5 $\begin{aligned} & \text { other HCCs vs NT }=1.3 \mathrm{E}-11 \\ & \text { PP vs other HCCs }=1.3 \mathrm{E}-11\end{aligned}$

NT PP other HCCs

$P=$
PP vs NT $=0.19$

OTC Pther HCCs vs NT $=1.3 \mathrm{E}-11$

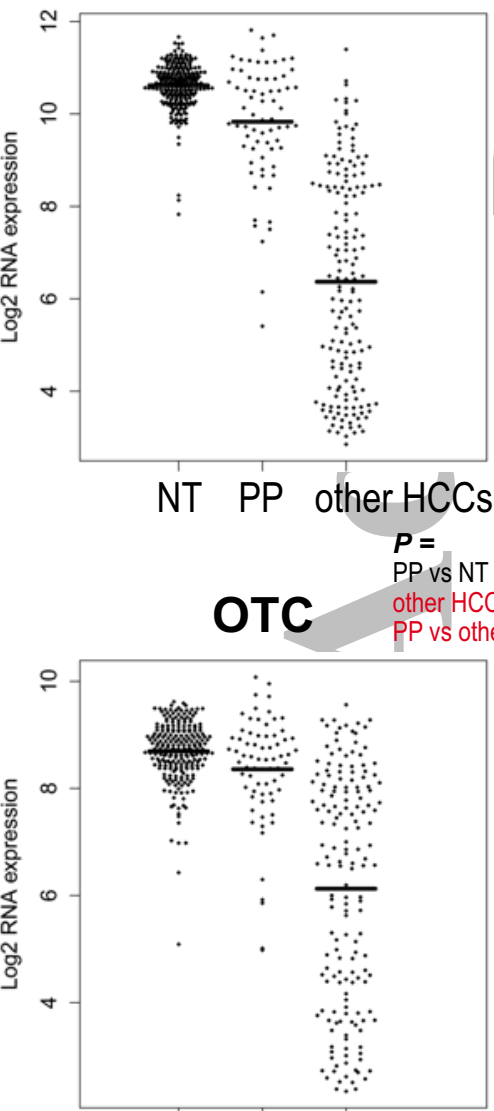

NT PP other HCCs

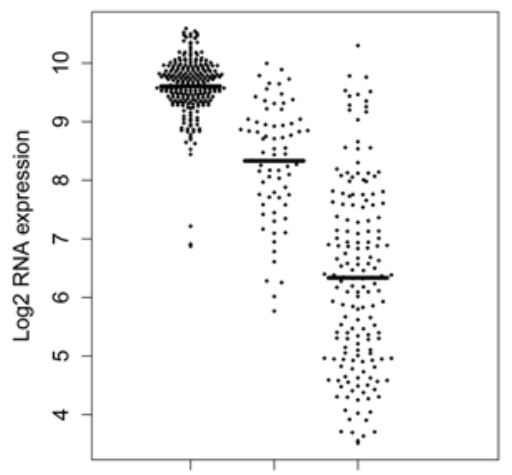

NT PP other HCCs

Hepatology

This article is protected by copyright. All rights re Désert et al., Supporting Figure 15 


\section{University Library}

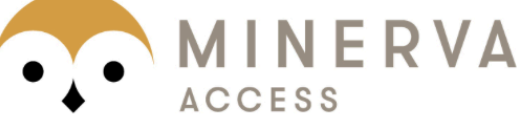

A gateway to Melbourne's research publications

Minerva Access is the Institutional Repository of The University of Melbourne

\section{Author/s:}

Desert, R;Rohart, F;Canal, F;Sicard, M;Desille, M;Renaud, S;Turlin, B;Bellaud, P;Perret, C;Clement, B;Le Cao, K-A;Musso, O

Title:

Human Hepatocellular Carcinomas With a Periportal Phenotype Have the Lowest Potential for Early Recurrence After Curative Resection

Date:

2017-11-01

Citation:

Desert, R., Rohart, F., Canal, F., Sicard, M., Desille, M., Renaud, S., Turlin, B., Bellaud, P., Perret, C., Clement, B., Le Cao, K. -A. \& Musso, O. (2017). Human Hepatocellular Carcinomas With a Periportal Phenotype Have the Lowest Potential for Early Recurrence After Curative Resection. HEPATOLOGY, 66 (5), pp.1502-1518. https://doi.org/10.1002/ hep.29254.

Persistent Link:

http://hdl.handle.net/11343/293504 